\title{
COMPREHENSIVE INSOMNIA ASSESSMENT FOLLOWING MILD TRAUMATIC BRAIN INJURY
}

by

Dora M. Zalai

Master of Arts, Ryerson University, 2012

Dipl. Psych, The Open University, 2005

Doctor of Medicine, Semmelweis University, 1998

\author{
A dissertation presented to Ryerson University \\ in partial fulfillment of the \\ requirements for the Degree of Doctor of Philosophy \\ in the program of Psychology \\ Toronto, Ontario, Canada, 2018
}

(C) Dora M. Zalai, 2018 


\section{Author's Declaration for the Electronic Submission of the Dissertation}

I hereby declare that I am the sole author of this dissertation. This is a true copy of the dissertation, including any required final revisions, as accepted by my examiners.

I authorize Ryerson University to lend this dissertation to other institutions or individuals for the purpose of scholarly research.

I further authorize Ryerson University to reproduce this dissertation by photocopying or by other means, in total or in part, at the request of other institutions or individuals for the purpose of scholarly research.

I understand that my dissertation may be made electronically available to the public. 
Comprehensive Insomnia Assessment Following Mild Traumatic Brain Injury

Doctor of Philosophy, 2018

Dora M. Zalai

Psychology

Ryerson University

\begin{abstract}
Background and Rationale: Insomnia symptoms following mild traumatic brain injury (mTBI) predict poor TBI outcomes. Insomnia symptoms may be caused by sleep disorders that can be effectively treated, which in turn, may improve mTBI outcomes. Previous studies have focused on insomnia symptom assessment in mTBI or evaluated samples with all TBI severities. To effectively manage insomnia following mTBI, it is important to understand which sleep disorders contribute to insomnia symptoms in this clinical group. Furthermore, it is important to extend research on primary insomnia to determine which variables are related to the perception of poor sleep among individuals who report new onset/worsening insomnia symptoms following mTBI. Objectives: (1) determine the prevalence of sleep disorders that contribute to chronic insomnia symptoms in patients with mTBI and (2) determine which objectively measured electroencephalographic and subjective variables are associated with subjective wake time and the perception of poor sleep among patients with chronic insomnia symptoms following mTBI. Methods: Individuals with chronic insomnia symptoms following mTBI $(N=50$; age 17-65; 64\% females; 3 - 24 months post mTBI) participated in a multi-method sleep and circadian assessment. Sleep disorders were diagnosed according to ICSD-3 criteria. Results: Insomnia disorder was the most common diagnosis (62\%), followed by obstructive sleep apnea (OSA) -
\end{abstract}


44\%; circadian rhythm sleep-wake disorders (CRSWD) - 26\% and periodic limb movement disorder (PLMD) - 8\%. The overestimation of wake time was similar to what has been described in primary insomnia. In contrast to the REM instability hypothesis of primary insomnia, REM sleep duration was not related to subjective wake time. Both low sleep quality and feeling unrested in the morning had the strongest relationship to subjective wake time. Feeling unrested was also associated with anxiety. Conclusions: OSA and CRSWD frequently occur among patients whose main presenting sleep symptom is chronic insomnia following a mTBI. Accordingly, objective sleep and circadian assessment should be part of chronic insomnia evaluation following a mTBI. The results imply that interventions reducing subjective wake time and anxiety could improve subjective sleep quality; however, these interventions should be implemented in conjunction with the treatment for OSA, CRSWD and PLMD. 


\section{Acknowledgements}

I thank the following people for their contribution to this research study and dissertation:

Dr. Todd Girard and Dr. Michael Cusimano - for accepting me as their PhD their student; for their interest in this research project and for the education and support they have provided. I look forward to collaborating with them in the future.

Dr. Colin Shapiro - for providing clinical consultation and mentorship

Dr. Laszlo Osvath - for consultation about the home PSG studies, for the home PSG analyses and for providing the level of consciousness analyses

Dr. Lixia Yang - for her comments and suggestions on the dissertation proposal and the dissertation

Dr. Nada Huterer and Dr. Naheed Hossain - for scoring the PSG studies and providing quality control

Bojana Gladanac and Dr. Sharon Chung - for conducting the melatonin assays

Emma van Reekum and Laurel Etkin-Spigelman - for their help with the lab PSG recordings and data entry

Zohra Kakar and Julie Abdurahman - for their help with the laboratory PSG studies

The sleep technicians and sleep physicians of the Sleep and Alertness Clinic - for scoring and evaluating the laboratory PSG studies

The technicians of the Melatonin Testing Laboratory - for their help with the data collection

The management of the Sleep and Alertness Clinic and the Melatonin Testing Lab - for their support of the research study

Dr. Rafael Pelayo, Dr. Stephanie Cassin and Dr. Oona St-Amant - for their questions and comments on the dissertation

This study was supported by the Canadian Institute of Health Research, the Youthdale Sleep Foundation and NeuroZone MSH. 
To my mother 


\section{Table of Contents}

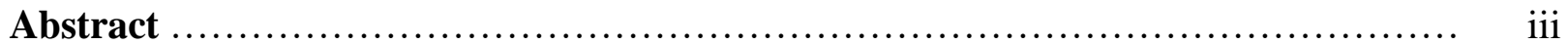

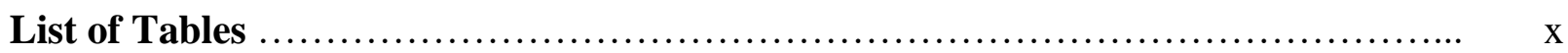

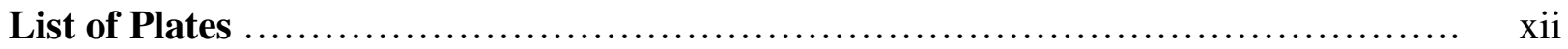

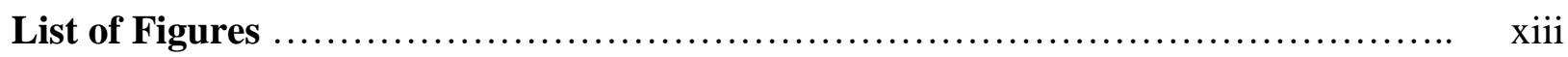

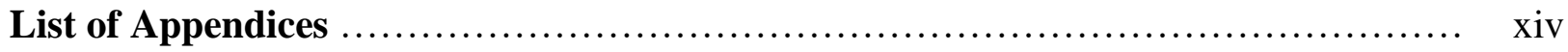

Chapter 1: Introduction............................................... 1

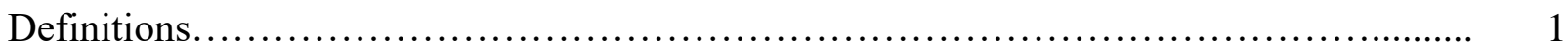

Insomnia Assessment...................................................... 9

Insomnia in Traumatic Brain Injury............................................ 13

Rationale for the Present Study........................................... 28

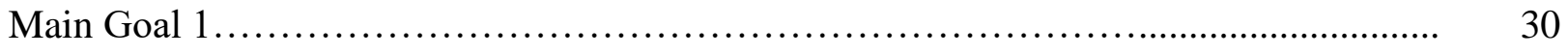

Main Goal 2.......................................................................... 33

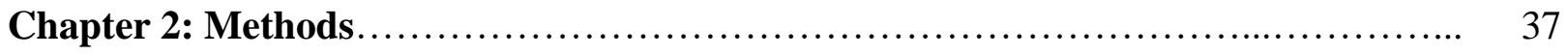

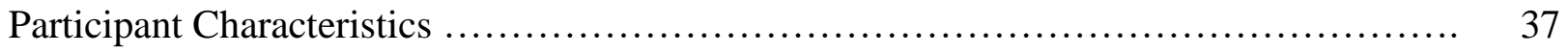

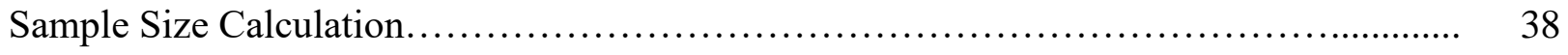

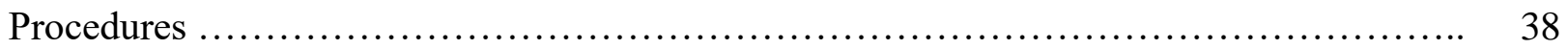

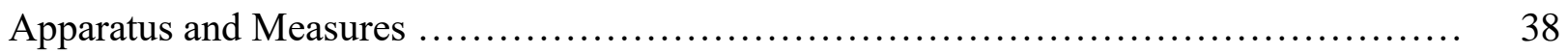

Chapter 3: Description of Participant Characteristics, Questionnaire Scores, Sleep

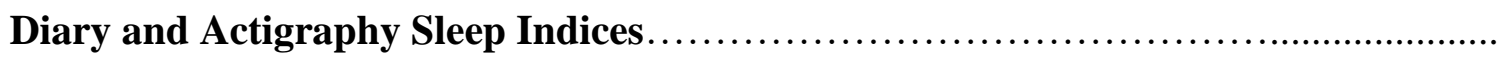

Analyses of Participant Characteristics, Questionnaire Scores, Sleep Diary and

Actigraphy Sleep Indices.

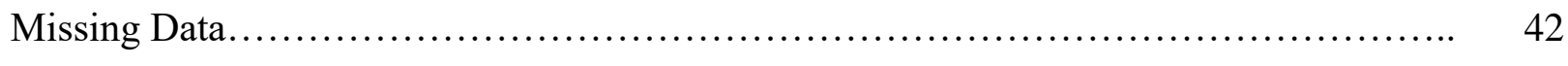

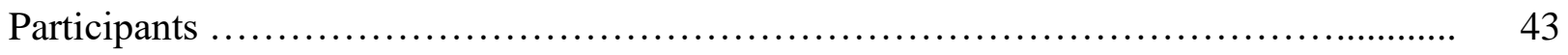


Questionnaire Scores ........................................................... 47

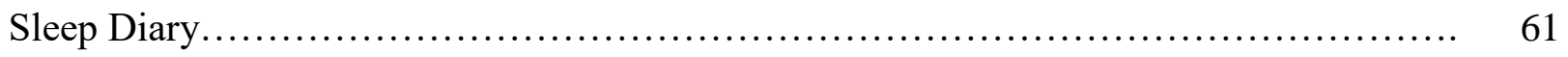

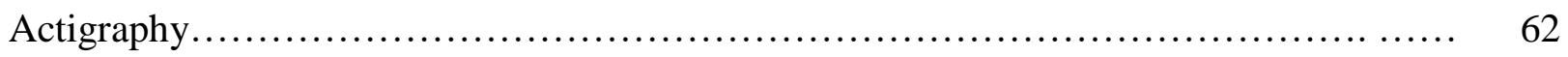

Correlation between Sleep Diary and Actigraphy Sleep Indices....................... 62

Summary of Questionnaire, Actigraphy, and Sleep Diary Results........................... 64

Chapter 4: The Use of Polysomnography in the Diagnostic Assessment of Chronic

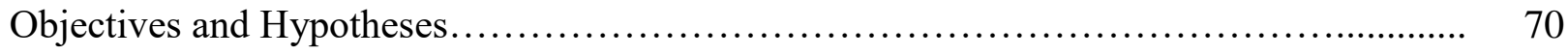

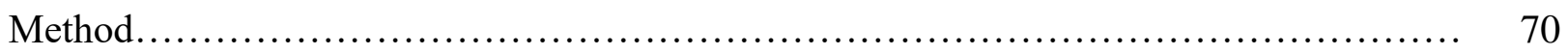

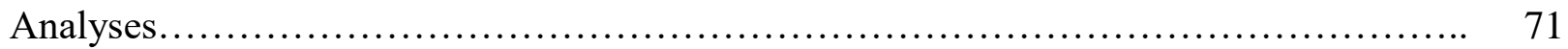

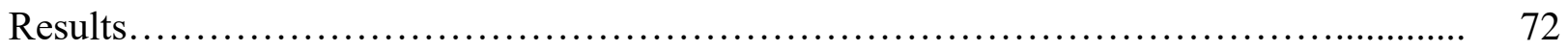

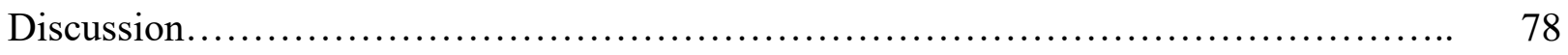

Chapter 5: Circadian Sleep-Wake Phase Disorders among Individuals with Chronic Insomnia Following Mild Traumatic Brain Injury .....................

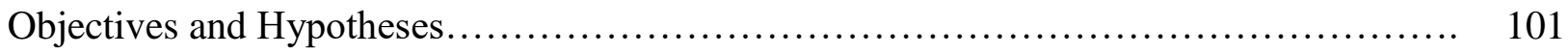

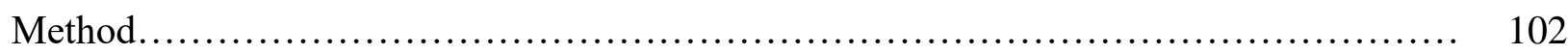

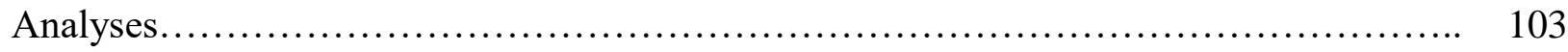

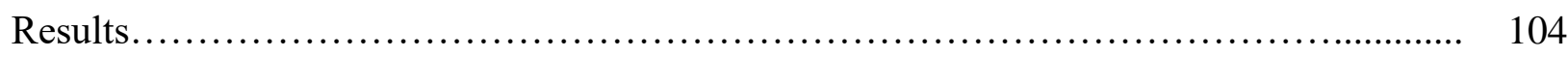

Discussion............................................................................. 109

Chapter 6: Testing the REM Instability Hypothesis in Chronic Insomnia Following Mild Traumatic Brain Injury

Objectives and Hypotheses...................................................... 123 
Method.

Analyses.

124

Results

Discussion

130

Chapter 7: Sleep Quality and Non-Restorative Sleep in Chronic Insomnia following Mild Traumatic Brain Injury

Objectives and Hypotheses.

Method.

Analyses.

Results

Discussion.

Goal 1: Diagnostic Findings.

Goal 2: Findings Related to Sleep Perception

Additional Results.

Study Limitations.

163

Future Directions

164

Conclusions.

Appendices

References.

Glossary 


\section{List of Tables}

Table 1.1. Insomnia Assessment Methods................................ 11

Table 1.2. Studies Using Standard Sleep Disorder Diagnostic Criteria in Traumatic Brain Injury....................................................

Table 1.3. Prevalence of Sleep Disorders....................................... 18

Table 1.4. Insomnia Assessment in Samples that Included Participants with MTBI... 21

Table 1.5. Possible Predisposing, Precipitating and Perpetuating Factors of Chronic Insomnia Following Traumatic Brain Injury..........................

Table 2.1. Study Procedures............................................ 39

Table 3.1. Missing Data................................................. 43

Table 3.2. Participant Characteristics..................................... 44

Table 3.3. Descriptive Characteristics of the Study Questionnaires................. 48

Table 3.4. Insomnia Severity Categories Based on ISI Scores $\ldots \ldots \ldots \ldots \ldots \ldots \ldots \ldots \ldots$

Table 3.5. Distribution of DASS scores.................................. 53

Table 3.6. Correlations Among the Insomnia Severity Index and Cognitive 59

Table 3.7. Average Visual Analogue Scale (VAS) Ratings...................... 61

Table 3.8. Sleep Diary Data.......................................... 62

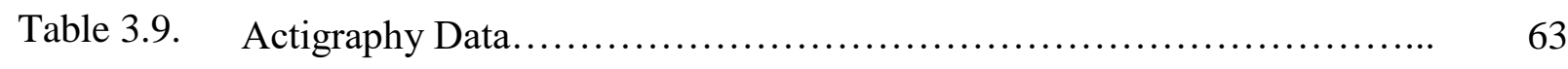

Table 4.1. Coefficients of the Model with STOP-BANG Score as a Predictor 76

Table 5.1. Summary of Studies Assessing Melatonin Secretion and Circadian SleepWake Disorders Following Traumatic Brain Injury.................... 


\section{List of Tables (Cont.)}

Table 5.2. Key Features of the Circadian Sleep-Wake Disorders Diagnosed in the Dissertation Study..................................................

Table 5.3. Relationship between Chronotype Categories and Circadian Sleep-Wake Disorders

Table 5.4. Comparison of DLMO and Sleep Timing between Patients Diagnosed with Insomnia Disorder and Delayed Phase Sleep-Wake Disorder......

Table 5.5. Correlations between DLMO and Sleep Timing Variables............... 108

Table 6.1. Descriptive Statistics of Proposed Predictor and Outcome Variables _ REM Instability Hypothesis

Table 6.2. Descriptive Statistics of the Arousal Index............................ 128

Table 7.1. Average VAS Ratings.......................................... 139

Table 7.2. VAS Sleep Quality and Feeling Rested Correlations..................... 142

Table 7.3. Correlations among ESS, FSS, and VAS Ratings..................... 145 


\section{List of Plates}

Panel 1.1. Definition of Mild Traumatic Brain Injury......................... 2

Panel 1.2. Insomnia Disorder Definitions................................... 4

Panel 1.3. Obstructive Sleep Apnea Definition.............................. 6

Panel 1.4. General Criteria for Circadian Rhythm Sleep-Wake Disorders............. 7

Panel 1.5. Periodic Limb Movement Disorder Definition........................ 8

Panel 1.6. Objectives and Hypotheses.................................... 36 


\section{List of Figures}

Figure 1.1. Insomnia Symptoms...................................... 9

Figure 1.2. Subjective and PSG Assessment of Sleep Disorders ............... 31

Figure 2.1. Study Procedures............................................ 41

Figure 3.1. Insomnia Severity Index (ISI) Score Distribution.................................. 50

Figure 3.2. Fatigue Severity Scale (FSS) Score Distribution................................ 50

Figure 3.3. STOP-BANG Questionnaire Score Distribution................................. 52

Figure 3.4. Depression Anxiety Stress Scale - Depression Subscale (DASS-D) Score Distribution... 54

Figure 3.5. Depression Anxiety Stress Scale - Anxiety Subscale (DASS-A) Score Distribution

Figure 3.6. Depression Anxiety Stress Scale - Stress Subscale (DASS-S) Score Distribution.

Figure 3.7. Dysfunctional Beliefs and Attitudes about Sleep Scale (DBAS) Score Distribution

Figure 3.8. Daytime Insomnia Symptom Response Scale (DISRS) Score Distribution

Figure 3.9. Anxiety and Preoocupation about Sleep Questionnaire (APSQ) Score Distribution.

Figure 3.10. Glasgow Sleep Effort Scale (GSES) Score Distribution.......................... $\quad 58$

Figure 3.11. Morningness -Eveningness Questionnaire (MEQ) Score Distribution...... 60

Figure 4.1. Possible Directions of Relationship between OSA and Insomnia......... 82

Figure 4.2. Theoretical Pathways between OSA and Insomnia Following MTBI....... 84

Figure 4.3. Undiagnosed OSA Manifests Following a MTBI......................... 85

Figure 8.1. Summary of the Sleep and Circadian Diagnostic Findings............... 150

Figure 8.2. Summary of the Sleep and Circadian Diagnostic Findings- Break-down of Specific Sleep Disorders

Figure 8.3. Overlapping Symptoms of Insomnia and MTBI. 


\section{List of Appendices}

Appendix A Explanation of Insomnia Disorder Definitions......................... 167

Appendix B Insomnia Assessment................................................. 170

Appendix C Description of Insomnia Assessment Studies that Included Participants 176 with Mild Traumatic Brain Injury....................................... 176

Appendix D Assessment Tools and Methods....................................... 181

Appendix E Circadian Sleep Regulation and the Effect of Melatonin on Sleep......... 194

Appendix F $\quad$ PSG Sleep Parameters .............................................. 197 


\section{Chapter 1: Introduction}

The purpose of this introduction is to describe the rationale and main objectives of this dissertation. More in-depth background for each study objective is provided in the individual chapters covering each of the specific objective. There are several tables referred to in the text that present detailed information about relevant studies. Interested readers can find information about relevant sleep and insomnia assessment methods and tools in the Appendices.

\section{Definitions}

Mild traumatic brain injury. Mild traumatic brain injury (mTBI) - a brain trauma caused by an external mechanical force - is the most common neurological condition, with an incidence rate of 600 in 100,000 individuals per year (Cassidy et al., 2004; Ryu, Feinstein, Colantonio, Streiner, \& Dawson, 2009; WHO, 2006). Although mortality resulting from mTBI is below one percent and only one-sixth to one half mTBI are treated in hospital, mTBI cases constitute the majority (70-90\%) of all treated traumatic brain injuries (Cassidy et al., 2004). The definition of mTBI is summarized in Panel 1.1 on the next page.

The acute symptoms of mTBI include headache, nausea, vomiting, visual and balance problems, dizziness, tinnitus, sensitivity to light or noise, drowsiness, fatigue, hypersomnia, insomnia, mood and cognitive problems. These usually subside within days or weeks. However, individuals with a history of mTBI may also report fatigue, headache, mood, and cognitive problems beyond three months (Carroll et al., 2004; Cassidy et al., 2014). If at least three symptoms persist or worsen following the injury, the diagnosis of post-concussion syndrome (International Classification of Diseases, 10th edition; ICD 10) or Neurocognitive Disorder due to Traumatic Brain Injury (Diagnostic and Statistical Manual of Mental Disorders, (DSM-5 ), 2013; WHO, 2010) can be considered. It is important to note that the subjective symptoms 
patients report after the acute phase of the injury are not specific to mTBI, although people tend to attribute the symptoms to the injury and may underreport the presence or severity of the same symptoms predating the injury (Davis, 2002; Lange, Iverson, \& Rose, 2010).

Persistent subjective post-concussion complaints are significant both from an individual and public health perspective since these symptoms impact quality of life and lead to functional impairment (ONF, 2013). Accordingly, both early identification of patients who are at high risk for poor prognosis and timely diagnosis of modifiable factors associated with persistent subjective post-traumatic symptoms are imperative.

Panel 1.1.

Definition of Mild Traumatic Brain Injury (Ruff, Iverson, Barth, Bush, \& Broshek, 2009)

A traumatically induced physiological disruption of brain function, as manifested by at least one of the following:

any loss of consciousness

any loss of memory for events immediately before or after the accident any alteration in mental state at the time of the accident (e.g., feeling dazed, disoriented, or confused) and focal neurologic deficit(s) that may or may not be transient

But:

loss of consciousness of less than approximately 30 minutes after 30 minutes, an initial Glasgow Coma Scale score of 13-15 and post-traumatic amnesia not greater than $24 \mathrm{hr}$ 
Insomnia. "Insomnia" is a broad, generic term that is used to label non-restorative, poor quality sleep or sleep difficulties such as difficulty falling asleep or staying asleep or waking up earlier than desired. Insomnia is also a short form of "insomnia disorder" defined by a set of symptoms that meet diagnostic criteria (Diagnostic and Statistical Manual of Mental Disorders, (DSM-5 ), 2013; International Classification of Sleep Disorders: Diagnostic and Coding Manual, 2014), see Panel 1.2 on page 4 and Appendix A.

Acute insomnia symptoms (difficulty with falling asleep or staying asleep or waking up earlier than usual) are normal and transient responses to stress. These symptoms often subside within approximately three months, unless sleep-specific non-adaptive cognitions or behaviours maintain the condition (Spielman, Caruso, \& Glovinsky, 1987). Insomnia symptoms can also be perpetuated by a variety of sleep disorders including obstructive sleep apnea (OSA), restless legs syndrome (RLS), periodic limb movement disorder (PLMD) or circadian rhythm sleep-wake disorders (CRSWD) as shown in Figure 1.1 on page 6. (Definitions of these disorders are shown in Panels 1.3, 1.4 and 1.5). A diagnosis of insomnia disorder is assigned only if the insomnia symptoms are not fully explained by another sleep disorder. This distinction is important from a clinical perspective because evidence-based treatments target specific sleep disorders rather than generic symptoms.

It is important to note that insomnia disorder requires assessment and treatment in its "own right" even if comorbid medical (including neurological and psychiatric) conditions are present. The current diagnostic systems assume that insomnia disorder is not simply a symptom of a medical condition. This is reflected by the change in classification from "primary" and "secondary" insomnia in DSM IV to "insomnia disorder" in DSM 5. 
Panel 1.2.

Insomnia Disorder Definitions

Insomnia Disorder (Diagnostic and Statistical Manual for Mental Disorders, 5th edition, 2013)

A. A predominant complaint of dissatisfaction with sleep quantity or quality, associated with one (or more) of the following symptoms:

1. Difficulty initiating sleep.

2. Difficulty maintaining sleep, characterized by frequent awakenings or problems returning to sleep after awakenings.

3. Early- morning awakening with inability to return to sleep.

B. The sleep disturbance causes clinically significant distress or impairment in social, occupational, educational, academic, behavioural, or other important areas of functioning.

C. The sleep difficulty occurs at least 3 nights per week.

D. The sleep difficulty is present at least 3 months.

E. The sleep difficulty occurs despite adequate opportunity to sleep.

F. The insomnia is not better explained by and occurs exclusively during the course of another sleep-wake disorder (e.g. narcolepsy, a breathing-related sleep disorder, a CRSWD, a parasomnia).

G. The insomnia is not attributable to the physiological effects of a substance (a drug of abuse, a medication).

H. Coexisting mental disorders and medical conditions do not adequately explain the predominant complaint of insomnia.

Specify if:

Episodic: Symptoms last at least 1 month but less than 3 months

Persistent: Symptoms last at least 3 months or longer

Recurrent: Two (or more) episodes within the space of 1 year 


\section{Chronic Insomnia Disorder (International Classification of Sleep Disorders, 3rd edition,}

2014)

Criteria A-F must be met

The patient reports, or the patient's parent or caregiver observes one or more of the following:

A. 1. Difficulty initiating sleep.

2. Difficulty maintaining sleep

3. Waking up earlier than desired

4. Resistance to going to bed on appropriate schedule

5. Difficulty sleeping without parent or caregiver intervention

B. The patient reports, or the patient's parent or caregiver observes, one or more of the following related to nighttime sleep difficulty:

1. Fatigue/malaise.

2. Attention, concentration, or memory impairment.

3. Impaired social, family, occupational, or academic performance.

4. Mood disturbance/irritability.

5. Daytime sleepiness.

6. Behavioural problems (e.g. hyperactivity, impulsivity, aggression).

7. Reduced motivation, energy, initiative.

8. Proneness for errors/accidents.

9. Concerns about or dissatisfaction with sleep.

C. The reported sleep/wake complaints cannot be explained purely by inadequate sleep opportunity (i.e. enough time is allotted for sleep) or inadequate circumstances (i.e. environment is safe, dark, quiet, and comfortable) for sleep.

D. The sleep disturbance and associated daytime symptoms occur at least three times per week.

E. The sleep disturbance and associated daytime symptoms have been present for at least 3 months.

F. The sleep disturbance is not better explained by another sleep disorder

Note: If the symptoms have been present for less than three months, short term insomnia disorder diagnosis is assigned. 
Panel 1.3. Obstructive Sleep Apnea Definition

Obstructive Sleep Apnea - Adult (International Classification of Sleep Disorders, 3rd

edition, 2014)

(A and B) or C to satisfy the criteria

A. The presence of one or more of the following

1. The patient complains of sleepiness, nonrestorative sleep, fatigue, or insomnia symptoms

2. The patient wakes with breath holding, gasping, or choking

3. The bed partner or other observer reports habitual snoring, breathing interruptions, or both during the patient's sleep

4. The patient has been diagnosed with hypertension, a mood disorder, cognitive dysfunction, coronary artery disease, stroke, congestive heart failure, atrial fibrillation, or type 2 diabetes mellitus.

B. Polysomnography (PSG) or Out of Center Sleep Testing (OCST) demonstrates

1. Five or more predominantly obstructive respiratory events (obstructive or mixed apneas, hypopneas, or respiratory effort related arousals per hour of sleep during a PSG or per hour of monitoring with OCST

OR

C. PSG or OCST demonstrates

1. Fifteen or more predominantly obstructive respiratory events (obstructive or mixed apneas, hypopneas, or respiratory effort related arousals per hour of sleep during a PSG or per hour of monitoring with OCST 
Panel 1.4. General Criteria for Circadian Rhythm Sleep-Wake Disorders

\section{General Criteria for Circadian Rhythm Sleep-Wake Disorder (International Classification}

\section{of Sleep Disorders, 3rd edition, 2014)}

A - C must be met

A. A chronic and recurrent pattern sleep-wake rhythm disruption primarily due to alteration of the endogenous circadian timing system or misalignment between the endogenous circadian rhythm and the sleep-wake schedule desired or required by the individual's physical environment or social/work schedules.

B. The circadian disruption leads to insomnia symptoms, excessive sleepiness, or both.

C. The sleep and wake disturbances cause significant distress or impairment in mental, physical, social, occupational, educational, or other important areas of functioning. 
Panel 1.5. Periodic Limb Movement Disorder Definition

Periodic Limb Movement Disorder (International Classification of Sleep Disorders, 3rd edition, 2014)

A - D must be met

A. PSG demonstrates periodic limb movements in sleep (PLMS), as defined in the most recent version of the AASM criteria.

B. The frequency is $>5 /$ hour in children and $>15 /$ hour in adults

C. The PLMS cause clinically significant sleep disturbance or impairment in mental, physical, social, occupational, educational, or other important areas of functioning.

D. The PLMS and the symptoms are not better explained by another current sleep disorder, medical or neurological disorder, or mental disorder (e.g. PLMS occurring with apneas or hypopneas should not be scored). 
Insomnia symptoms (sleep initiation and/or sleep maintenance difficulty, early morning awakening, non-restorative sleep)
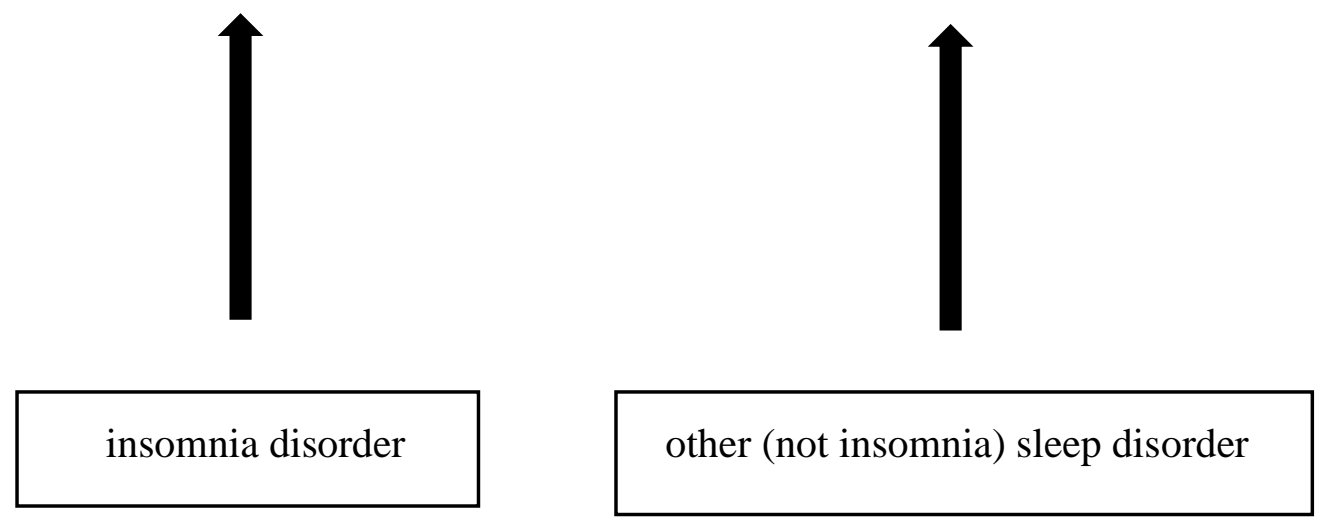

Figure 1.1. Insomnia Symptoms

Figure 1.1. shows that insomnia symptoms can be caused by various sleep disorders. The diagnosis of insomnia disorder is assigned only if other sleep disorders do not fully account for the insomnia symptoms. This distinction is important because insomnia disorder and other sleep disorders have different pathology and these disorders require different evidence - based treatment. Insomnia disorder can be comorbid with other sleep disorders and in these situations, treatment for both sleep disorders may be necessary.

\section{Insomnia Assessment}

Insomnia symptoms can be assessed with generic questionnaires, for example concussion questionnaires that include sleep items. A set of symptoms that comprise insomnia disorder can be assessed with validated, insomnia-specific questionnaires that have known cut-off scores to record clinically significant symptoms. Insomnia disorder diagnosis requires a clinical interview that assesses symptoms according to diagnostic criteria and rules out other sleep disorders that may give rise to insomnia symptoms (Schutte-Rodin, Broch, Buysse, Dorsey, \& Sateia, 2008). This can be supplemented with other measures, for example a sleep diary, which provides prospective and numerical subjective data of key sleep variables, or with questionnaires that 
assess insomnia symptom severity or measure sleep-specific psychological factors that may perpetuate the condition.

Objective measures - for example polysomnography (PSG) and actigraphy - are used to diagnose other, non-insomnia sleep disorders that may cause/amplify insomnia symptoms. The use of objective assessment tools - in particular, PSG - is considered especially important for differential diagnosis in clinical populations, where the base rate of sleep disorders that can cause insomnia symptoms is high.

Given that approximately one third of patients in various medical populations have clinically significant insomnia symptoms based on screening instruments and that the rate of non -insomnia sleep disorders appears to be high in some medical populations, it would be desirable to evaluate the clinical utility of PSG in the diagnostic assessment of insomnia among these different clinical groups (Budhiraja, Roth, Hudgel, Budhiraja, \& Drake, 2011). Circadian evaluation, including actigraphy and the assessment of melatonin secretion are far from standard insomnia evaluation procedure but are merited in a few clinical groups where insomnia caused by abnormal melatonin secretion is common, for example children with fetal alcohol syndrome or individuals with ADHD (Goril, Zalai, Scott, \& Shapiro, 2016; Van Veen, Kooij, Boonstra, Gordijn, \& Van Someren, 2010). For more information about insomnia assessment, please see Table 1.1 on the next page and Appendix B. 
Table 1.1.

Insomnia Assessment Methods

\begin{tabular}{|c|c|c|}
\hline Method & Advantages & Disadvantages \\
\hline Questionnaires & $\begin{array}{l}\text { Can be completed in a few minutes } \\
\text { Can be used for: } \\
\text { screening } \\
\text { symptom severity assessment before, during and } \\
\text { after treatment } \\
\text { identifying psychological factors that can be } \\
\text { treatment targets; for example, dysfunctional } \\
\text { attitudes and beliefs } \\
\text { Inexpensive }\end{array}$ & $\begin{array}{l}\text { Retrospective and global } \\
\text { Several measures are not validated for } \\
\text { different clinical populations } \\
\text { Most psychological measures do not have a } \\
\text { clinical cut-off } \\
\text { It is unknown if the psychological measures } \\
\text { differentiate between insomnia and other } \\
\text { sleep disorders }\end{array}$ \\
\hline Semi-structured interviews & $\begin{array}{l}\text { Detailed assessment according to diagnostic criteria } \\
\text { Provides information for differential diagnosis } \\
\text { Higher inter-assessor reliability than unstructured } \\
\text { clinical interviews }\end{array}$ & $\begin{array}{l}\text { Requires training and knowledge of sleep } \\
\text { disorders } \\
\text { Gathers retrospective and global subjective } \\
\text { information }\end{array}$ \\
\hline
\end{tabular}




\begin{tabular}{|c|c|c|}
\hline Method & Advantages & Disadvantages \\
\hline \multirow[t]{3}{*}{ Sleep diary } & $\begin{array}{l}\text { Provides prospective information on bedtime, rising } \\
\text { time, awakenings and perceived sleep quality }\end{array}$ & $\begin{array}{l}\text { Calculated sleep parameters are based on } \\
\text { subjective data }\end{array}$ \\
\hline & $\begin{array}{l}\text { SOL, WASO, total wake time, and SE can be } \\
\text { calculated from the data }\end{array}$ & $\begin{array}{l}\text { Only small-moderate convergent validity } \\
\text { with PSG }\end{array}$ \\
\hline & High ecological validity (completed at home) & \\
\hline \multirow[t]{2}{*}{ Wrist actigraphy } & Provides prospective data on sleep and wake & Low sensitivity of detecting wake time \\
\hline & $\begin{array}{l}\text { Useful for estimation of TST and WASO and } \\
\text { circadian sleep patterns }\end{array}$ & $\begin{array}{l}\text { Limited applicability for individuals with } \\
\text { paralysis or very sedentary lifestyle }\end{array}$ \\
\hline \multirow[t]{4}{*}{ Laboratory Polysomnography } & Provides objective measures of sleep and other & Expensive \\
\hline & physiological parameters & Requires trained staff \\
\hline & & Low ecological validity \\
\hline & $\begin{array}{l}\text { Essential for the diagnosis of a number of sleep } \\
\text { disorders }\end{array}$ & First night effect \\
\hline \multirow{7}{*}{$\begin{array}{l}\text { Ambulatory } \\
\text { Polysomnography }\end{array}$} & All advantages of lab PSG, plus high ecological & Higher risk of missing or invalid data \\
\hline & validity & because staff is not present during the \\
\hline & Does not necessitate trained staff to record the data & recording \\
\hline & Cheaper than lab PSG, allowing multiple & \\
\hline & assessments & Video monitoring may not be available \\
\hline & Can be used in locations where there are no sleep & \\
\hline & labs & \\
\hline
\end{tabular}




\section{Insomnia in Traumatic Brain Injury}

Disturbances of sleep and wakefulness have been detected in approximately $50 \%$ of patients following TBI; however, this estimation is based on weighted mean proportions from diverse studies with regards to definitions of sleep problems, assessment methods and tools, TBI severity, time since injury and the presence/absence of subjective sleep complaints (Mathias \& Alvaro, 2012). Most studies assessed symptoms only - based on this data, the most common sleep symptoms are snoring (60\%) and insomnia (50\%) among patients who suffered a TBI.

Insomnia symptoms are more frequently reported by people who had a mTBI compared to those who had a more severe TBI (Beetar, Guilmette, \& Sparadeo, 1996; Clinchot, Bogner, Mysiw, Fugate, \& Corrigan, 1998; Ouellet, Beaulieu-Bonneau, \& Morin, 2006). The prevalence of insomnia symptoms - based on Pittsburgh Sleep Quality Index score $\geq 5$ - is approximately $65 \%, 54 \%, 46 \%$, and $41 \%$ at two weeks, one month, six months, and 12 months following a mTBI (Theadom et al., 2015). Importantly, people who report insomnia symptoms following a mTBI will have more difficulties with social and community integration; have poorer cognition; more chronic post-concussion symptoms and behavioural difficulties; and more self-reported symptoms of depression and anxiety on the long term than individuals who sleep well following the brain injury (Mollayeva, Shapiro, Mollayeva, Cassidy, \& Colantonio, 2015; Theadom et al., 2015; Theadom et al., 2016).

Given that insomnia symptoms and snoring (a symptom of OSA) are reported by 50-60\% of individuals after TBI (all severities, Mathias \& Alvaro, 2012), it is surprising that only five of the 21 studies included in the meta-analysis on this subject provided sleep disorder diagnosis according to clinical diagnostic criteria, see Table 1.2. (Baumann, Werth, Stocker, Ludwig, \& 
Bassetti, 2007; Castriotta et al., 2007; Quellet \& Morin, 2006; Rao, Bergey, Hill, Efron, \& McCann, 2011; Verma, Anand, \& Verma, 2007). 
Table 1.2. Studies using Standard Sleep Disorder Diagnostic Criteria in Traumatic Brain Injury

\begin{tabular}{|c|c|c|c|c|c|}
\hline Study & Participants & TBI severity & Time since injury & Diagnoses & Comments \\
\hline \multirow[t]{2}{*}{$\begin{array}{l}\text { Baumann et al. } \\
\text { (2007) }\end{array}$} & $\begin{array}{l}76 \text { adults } \\
\text { Not selected based } \\
\text { on current sleep } \\
\text { problems } \\
\text { Patients with pre- } \\
\text { TBI sleep problems } \\
\text { were excluded }\end{array}$ & $\begin{array}{l}\text { 40\%: mTBI } \\
\text { 23\%: moderate } \\
\text { TBI } \\
\text { 37\%: severe TBI }\end{array}$ & Six months & $\begin{array}{l}\text { Most common } \\
\text { symptoms were } \\
\text { hypersomnia and } \\
\text { excessive daytime } \\
\text { sleepiness }(28 \%) \text {. } \\
\text { Disorders: } \\
\text { Insomnia: 5\% } \\
\text { OSA: 9\% } \\
\text { PLM: } 8 \% \\
\text { One patient with } \\
\text { mild and one with } \\
\text { severe TBI had } \\
\text { narcolepsy without } \\
\text { cataplexy. }\end{array}$ & $\begin{array}{l}\text { The authors } \\
\text { commented that the } \\
\text { low percentage of } \\
\text { insomnia compared } \\
\text { to other studies may } \\
\text { be because studies } \\
\text { that used } \\
\text { questionnaires only } \\
\text { overestimated the } \\
\text { prevalence of } \\
\text { insomnia disorder. }\end{array}$ \\
\hline & & & & $\begin{array}{l}\text { No circadian sleep } \\
\text { disorder diagnosis. }\end{array}$ & \\
\hline
\end{tabular}




\begin{tabular}{|c|c|c|c|c|c|}
\hline Study & Participants & TBI severity & Time since injury & Diagnoses & Comments \\
\hline $\begin{array}{l}\text { Castriotta et al, } \\
2007\end{array}$ & $\begin{array}{l}87 \text { patients with } \\
\text { TBI } \\
\text { Individuals who } \\
\text { may have had } \\
\text { circadian sleep } \\
\text { disorders and the } \\
\text { ones who were on } \\
\text { hypnotic } \\
\text { medications were } \\
\text { excluded }\end{array}$ & $\begin{array}{l}8 \%: \text { mTBI } \\
92 \%: \text { moderate \& } \\
\text { severe TBI }\end{array}$ & $\begin{array}{l}\text { At least three } \\
\text { months }\end{array}$ & $\begin{array}{l}20 \% \text { : OSA } \\
\text { 11\%: hypersomnia } \\
6 \% \text { : PLMS } \\
6 \% \text { : narcolepsy }\end{array}$ & $\begin{array}{l}\text { Insomnia disorder } \\
\text { was not reported }\end{array}$ \\
\hline Quellet et al. (2006) & $\begin{array}{l}14 \text { adults with } \\
\text { insomnia disorder } \\
\text { and } 14 \text { controls } \\
\text { Patients with } \\
\text { restless legs, high } \\
\text { risk for OSA and } \\
\text { PLMD were } \\
\text { excluded }\end{array}$ & $\begin{array}{l}\text { 29\%: mTBI } \\
7 \%: \text { mild-moderate } \\
29 \%: \text { moderate } \\
22 \%: \text { moderate- } \\
\text { severe TBI } \\
\text { 14\%: severe TBI }\end{array}$ & $\begin{array}{l}\text { seven months to } \\
\text { three years }\end{array}$ & $\begin{array}{l}\text { PSG: } \\
\text { Insomnia confirmed } \\
\text { in } 10 \text { patients (SOL } \\
\text { and or WASO) } \\
\text { All other PSG } \\
\text { parameters were } \\
\text { normal but } \\
\text { moderate-large } \\
\text { effects sizes for } \\
\text { WASO, SE, TST, } \\
\text { number of } \\
\text { awakenings > } 5 \text { min } \\
\text { between insomnia } \\
\text { and control groups. }\end{array}$ & $\begin{array}{l}\text { Insomnia disorder } \\
\text { assessment using } \\
\text { standard interview. }\end{array}$ \\
\hline
\end{tabular}




\begin{tabular}{|c|c|c|c|c|c|}
\hline Study & Participants & TBI severity & Time since injury & Diagnoses & Comments \\
\hline Rao et al (2011) & $\begin{array}{l}\text { Seven individuals } \\
\text { with mTBI and } \\
\text { controls }\end{array}$ & $\begin{array}{l}\text { Current sleep } \\
\text { problems were not } \\
\text { assessed before } \\
\text { inclusion but } \\
\text { individuals with } \\
\text { prior sleep disorder } \\
\text { diagnoses were } \\
\text { excluded }\end{array}$ & Not reported & $\begin{array}{l}\text { Six of the seven } \\
\text { individuals with } \\
\text { mTBI were } \\
\text { diagnosed with a } \\
\text { sleep disorder } \\
\text { (OSA or PLM). }\end{array}$ & $\begin{array}{l}\text { PSG was conducted } \\
\text { but clinical } \\
\text { interview was not } \\
\text { provided. } \\
\text { Current subjective } \\
\text { sleep problems or } \\
\text { symptoms were not } \\
\text { assessed/reported } \\
\text { Insomnia disorder } \\
\text { was not assessed }\end{array}$ \\
\hline Verma et al. (2007) & $\begin{array}{l}60 \text { adults } \\
\text { with sleep -related } \\
\text { complaints }\end{array}$ & $\begin{array}{l}40 \%: \text { mild } \\
18 \%: \text { moderate } \\
42 \%: \text { severe }\end{array}$ & $\begin{array}{l}\text { Between three } \\
\text { months to two years }\end{array}$ & $\begin{array}{l}\text { Symptoms: } \\
\text { Excessive daytime } \\
\text { sleepiness: } 50 \% \\
\text { Insomnia: } 33 \% \\
\text { Diagnoses: } \\
\text { 30\%: sleep apnea } \\
\text { 25\%: parasomnia } \\
9 \text { of } 28 \text { patients } \\
\text { who had MSLT had } \\
\text { signs of narcolepsy } \\
\text { but clinical } \\
\text { symptoms were not } \\
\text { assessed/reported }\end{array}$ & $\begin{array}{l}54 \text { patients had } \\
\text { PSG } \\
\text { It was not clear if } \\
\text { sleep disorders } \\
\text { were diagnosed } \\
\text { according to } \\
\text { standard criteria. } \\
\text { Insomnia symptoms } \\
\text { were assessed but } \\
\text { insomnia disorder } \\
\text { diagnosis was not } \\
\text { reported. }\end{array}$ \\
\hline
\end{tabular}


Four of these studies included participants across the full spectrum of TBI severity and the fifth had a small $(N=7)$ mTBI sample. Three of the five studies did not assess current sleep problems as part of the inclusion criteria; one included patients with subjective sleep problems and one small treatment study selected individuals with insomnia disorder. Four of the five studies (including the one mTBI study with a sample size of seven) determined the percentage of selected sleep disorders. According to the meta-analysis the most common disorders of sleep and wakefulness were insomnia disorder (29\%) and hypersomnia (28\%), followed by OSA (25\%), periodic limb movements in sleep (19\%) and narcolepsy (4\%) based on these four studies- some were more prevalent than they are in the general population, see Table 1.3.

Table 1.3.

Prevalence of Sleep Disorders

TBI Studies General Population

\begin{tabular}{lll}
\hline Insomnia Disorder & $29 \%$ & $10 \%$ \\
OSA & $25 \%$ & $24 \%$ in men and $9 \%$ in women \\
PLMD & $19 \%$ & $\begin{array}{l}\text { Rare below the age of } 40 ; \\
\text { common }(45 \%) \text { in older adults } \\
\text { Narcolepsy }\end{array}$ \\
& $4 \%$ & $\begin{array}{l}0.02 \%-0.18 \% \\
4-9.5 \% \text { of adults have SOREMs }\end{array}$ \\
& & during random MSLT \\
\hline
\end{tabular}

Note: The TBI data is derived from four studies based on a meta-analysis (Mathias \& Alvaro, 2012). The general population data is based on the ICSD 3. The inclusion criteria in terms of current sleep problems were heterogeneous. Not all studies assessed all sleep disorders and in some, diagnosis was based solely on PSG. OSA = obstructive sleep apnea, PLMD = periodic limb movement disorder, SOREM: Sleep onset REM; MSLT = multiple sleep latency test

However, the conclusions one can draw from this meta-analysis is limited because it summed the results of studies that had different inclusion and exclusion criteria with regards to sleep symptoms and prior sleep disorder diagnoses and included selective or incomplete 
assessment of sleep disorders. For example, two of the above studies did not assess insomnia symptoms and a third study assessed symptoms but did not provide insomnia disorder diagnosis, leaving only two studies that provided both symptom assessment and clinical judgement about insomnia disorder (Baumann et al., 2007; Quellet \& Morin, 2006). In both studies, patients with mTBI were under-represented. A further limitation is that CRSWD were specifically assessed (using actigraphy) only in one study; but this study involved patients with all TBI severities and 95\% of participants did not have insomnia symptoms (Baumann et al., 2007).

Only case reports and one study conducted a comprehensive diagnostic assessment (including actigraphy, melatonin assessment, and clinical interview) of CRSWD. This study diagnosed CRSWD in each of the 15 individuals whom were selected from a group of 42 mTBI patients with chronic insomnia at a sleep clinic (Ayalon, Borodkin, Dishon, Kanety, \& Dagan, 2007). This implies that approximately one third of mTBI patients who are referred to chronic insomnia evaluation may have a CRSWD; however, the limitations of the study (selection criteria for circadian assessment was not provided, age range was limited to young and middleaged adults, time since injury was not reported) restrict the generalizability of the results. CRSWD may have been labelled as insomnia or hypersomnia in other studies without accurate diagnostic assessment. Given that CRSWD and sleep disorder have specific and effective treatments, there is a need for a study that rigorously evaluates these disorders in a homogeneous group of patients with mTBI and chronic insomnia selected based on well-defined inclusion criteria to avoid the limitations of these studies cited above.

In summary, the limitation of the literature on insomnia after mTBI is that the research has focused almost exclusively on symptom assessment; see Table 1.4 on the next page and Appendix C. Only two studies diagnosed insomnia disorder and differentiated it from other sleep 
disorders, but these studies included patients with all severities of TBI and only a minority of participants had a mTBI (Table 1.2.). There is a need for studies that either involve samples with only a single TBI severity or separate analyses for different TBI severities should be provided. This is important because - in addition to the higher frequency of insomnia complaints among people with a history of mild versus severe TBI - there may also be differences with regards to the pathophysiology and psychopathology of insomnia between groups with varying TBI severities. Specifically, the brain injury may have a more direct anatomical/neuropathological contribution to insomnia in severe brain injury because the injury may directly cause damage in brain areas and neuronal circuits that orchestrate sleep. In contrast, sleep-specific beliefs may have a relatively larger role following a mild injury because of the higher insight, capacity for self-reflection, and expectations for rapid, full recovery among people who had a mild TBI. Previous research suggests that the contribution of various sleep disorders may be substantial across the spectrum of TBI severities but to establish this link properly, one needs to select patients based on well-defined insomnia criteria and conduct a comprehensive, evidence-based assessment of sleep and circadian disorders that can cause insomnia symptoms in patients with different TBI severities (Mathias \& Alvaro, 2012). The acceptability, feasibility, and measurement properties of sleep assessment tools may also be different in mild versus more severe TBI, which also underscores the need to work with samples that are clearly delineated in terms of TBI severity. 
Table 1.4.

Summary of the Types of Insomnia Assessment Performed in Studies that Included Participants with Mild Traumatic Brain Injury Page 1/3

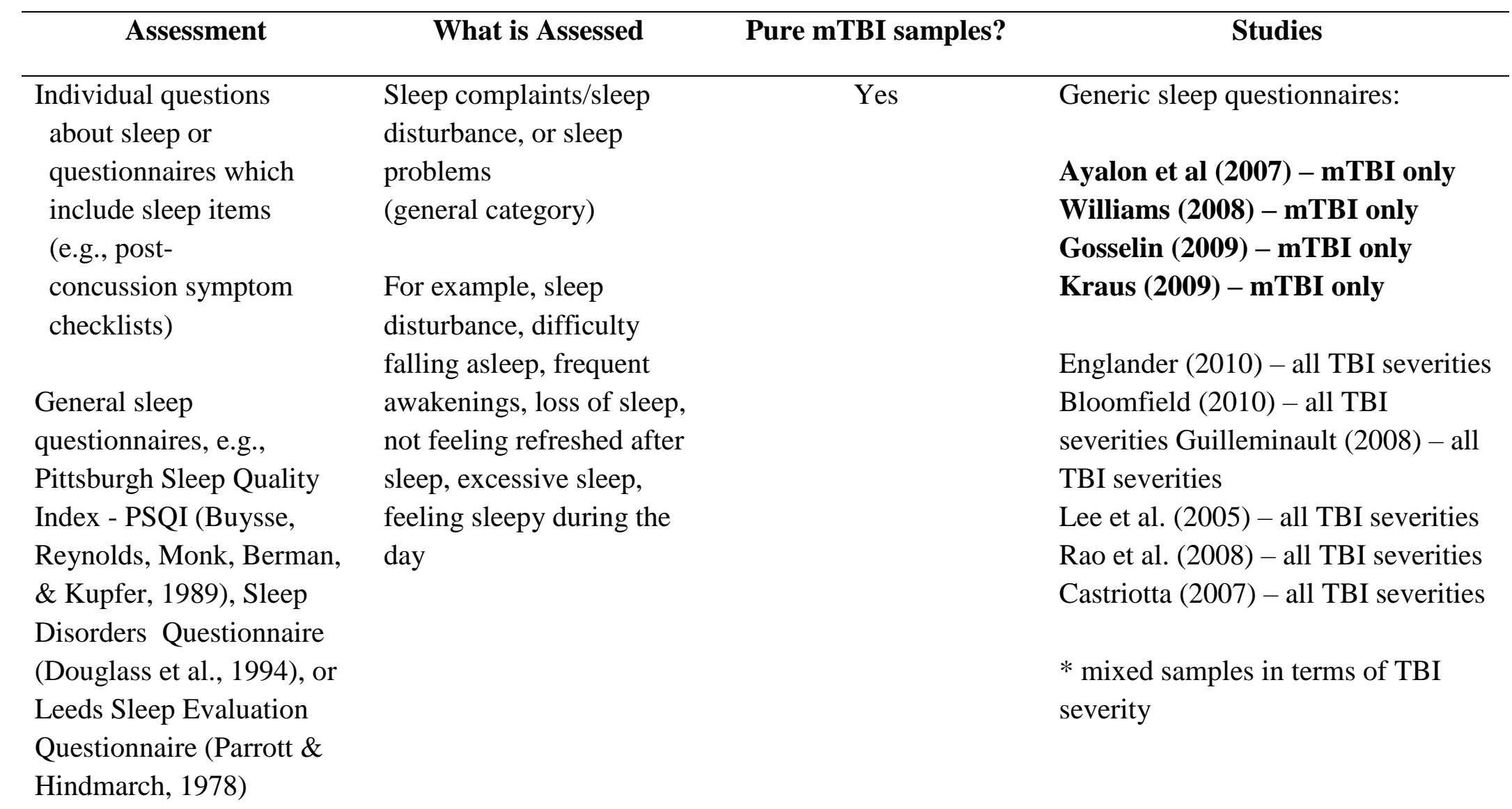




\begin{tabular}{|c|c|c|c|}
\hline Assessment & What is Assessed & Pure mTBI samples? & Studies \\
\hline $\begin{array}{l}\text { Insomnia symptom } \\
\text { questionnaires; e.g., } \\
\text { Insomnia Severity Index } \\
\text { (ISI) (Morin, 1993a) } \\
\text { Pittsburgh Sleep Quality } \\
\text { Index }{ }^{1}\end{array}$ & $\begin{array}{l}\text { Insomnia Symptoms, } \\
\text { i.e. difficulty falling } \\
\text { asleep, maintaining sleep, } \\
\text { or waking up earlier than } \\
\text { desired, and associated } \\
\text { daytime symptoms, } \\
\text { distress and functional } \\
\text { impairment }\end{array}$ & Yes & $\begin{array}{l}\text { Three studies used the PSQI and } \\
\text { differentiated between "poor sleep" } \\
\text { (PSQI > 5) and insomnia (PSQI > 8). } \\
\text { One of these studies involved a } \\
\text { mTBI sample (Theadom, 2015), the } \\
\text { other two involved patients with all } \\
\text { TBI severities (Fichtenberg, 2001; } \\
\text { Lu et al., 2015). } \\
\text { Five studies used the ISI (Ouellet, } \\
\text { 2006a, 2006b, 2007; Bloomfield, } \\
\text { 2010; Mollayeva, 2015). } \\
\text { Only one study involved a pure } \\
\text { mTBI sample and it used the ISI as } \\
\text { a continuous symptom measure } \\
\text { (Mollayeva, 2015). } \\
\text { In the remaining studies, the majority } \\
\text { of participants had moderate or } \\
\text { severe TBI. }\end{array}$ \\
\hline
\end{tabular}




\begin{tabular}{|c|c|c|c|}
\hline Assessment & What is Assessed & Pure mTBI samples? & Studies \\
\hline $\begin{array}{l}\text { Questionnaires } \\
\text { Clinical interview } \\
\text { Sleep diary } \\
\text { Actigraphy } \\
\text { Polysomnography }\end{array}$ & $\begin{array}{l}\text { Insomnia Disorder } \\
\text { (clinical diagnosis) i.e. } \\
\text { insomnia disorder } \\
\text { symptoms (as above) } \\
\text { meeting standard } \\
\text { diagnostic criteria and } \\
\text { cannot be fully attributed } \\
\text { to another sleep disorder }\end{array}$ & No & $\begin{array}{l}\text { Three studies included } 1,4 \text { and } 5 \\
\text { patients with mTBI and insomnia } \\
\text { disorder respectively, the other } \\
\text { participants has moderate or severe } \\
\text { TBI (Ouellet 2006, 2007; } \\
\text { Bloomfield, 2010). } \\
\text { One study involved } 116 \text { participants } \\
\text { and } 84.5 \% \text { had mTBI. Insomnia was } \\
\text { diagnosed in 55 \% of the sample, } \\
35 \% \text { had apnea, 20\% had PLM. Only } \\
\text { patients who were suspected to have } \\
\text { a sleep apnea had a PSG (Collen et } \\
\text { al., 2012). } \\
\text { Another diagnostic study involved } \\
60 \text { adults and } 24 \% \text { of the sample had } \\
\text { mTBI. Insomnia disorder was } \\
\text { diagnosed in 15\%; 30\% had sleep } \\
\text { apnea and 35\% had PLM. PSG was } \\
\text { performed in two-thirds of the } \\
\text { sample (Verma, 2007). }\end{array}$ \\
\hline
\end{tabular}

Note: Studies including pure mTBI samples are bolded.

$\mathrm{TBI}$ = traumatic brain injury; PSG = polysomnography; PLM = periodic limb movement disorder; ISI = Insomnia Severity Index ${ }^{1}$ The Pittsburgh Sleep Quality Index PSQI (Buysse et al., 1989) is intended to assess "sleep quality" but it has also been recommended for use in insomnia research for screening and as an outcome measure. The construct validity of the scale has been recently questioned and it has been recommended that the scale is not used for insomnia screening (Hartmann, Carney, Lachowski, \& Edinger, 2015) 
None of the studies that diagnosed insomnia disorder to date has focused solely on patients who reported new onset/amplified chronic insomnia symptoms following a mTBI and none included a full sleep and circadian evaluation, including diagnostic clinical interview, questionnaires, sleep diary, PSG, actigraphy and melatonin measurement.

To understand the mechanisms that perpetuate insomnia symptoms following TBI, it is important to go beyond symptom assessment and examine the role of various sleep disorders and other variables (for example sleep -specific cognitive and behavioural factors, pain and psychiatric issues) and in the pathophysiology and psychopathology of insomnia; for a summary of possible contributing factors, please see Table 1.5. This table also shows which of the perpetuating factors were considered and evaluated in this thesis. 
Table 1.5.

Possible Predisposing, Precipitating, and Perpetuating Factors of Chronic Insomnia Following Traumatic Brain Injury

\begin{tabular}{|c|c|c|}
\hline Variable & Possible Role & $\begin{array}{c}\text { Did the present study } \\
\text { assess it? }\end{array}$ \\
\hline
\end{tabular}

Personality traits (e.g., neuroticism)

Predisposing factor

Predisposing factor

Sleep reactivity, i.e., a trait-like predisposition to react to stressful life events with insomnia. It has familiar aggregation and may have a genetic underpinning.

Focal brain damage and cell loss (moderate and severe injury) and/or functional or micro-structural injury (e.g., ionic influx, energy crisis, axonal injury, impaired neurotransmission, cell death) - in mTBI

Changes in the circadian rhythm and/or amount of melatonin secretion

Life events and situations that the person perceives to be stressful/being stressed. Examples are: TBI; being deprived of important social interactions (e.g. peer relationships); being in the hospital; absence from school/work/sport activities; loss of income; perceived loss of health, litigation procedures.

Precipitating factor
Precipitating/ Perpetuating factor

Precipitating factor (life events)

Perpetuating factor (being stressed)

No

No

No

Yes - dim light melatonin onset test

No

Yes (DASS 21) 


\begin{tabular}{|c|c|c|}
\hline Variable & Possible Role & $\begin{array}{l}\text { Did the dissertation } \\
\text { study assess it? }\end{array}$ \\
\hline $\begin{array}{l}\text { Pain. Acute pain may precipitate insomnia and directly } \\
\text { disrupt sleep. Coping behaviours related to chronic } \\
\text { pain (e.g., excessive rest) maintain insomnia. }\end{array}$ & Precipitating/ Perpetuating factor & $\begin{array}{c}\text { Yes } \\
\text { - daily VAS Pain ratings } \\
\text { before bedtime }\end{array}$ \\
\hline $\begin{array}{l}\text { Psychiatric conditions, for example depression, anxiety } \\
\text { disorders, and PTSD }\end{array}$ & Precipitating/ Perpetuating factors & $\begin{array}{l}\text { Yes } \\
\text { - semi-structured clinical } \\
\text { interview and DASS }\end{array}$ \\
\hline $\begin{array}{l}\text { Maladaptive behaviours that individuals with insomnia } \\
\text { engage in. These behaviours may overlap with coping } \\
\text { behaviours in chronic pain, depression, and fatigue. }\end{array}$ & Perpetuating factors & No \\
\hline $\begin{array}{l}\text { Cognitive factors, for example dysfunctional beliefs } \\
\text { and attitudes about sleep and rumination about daytime } \\
\text { symptoms of insomnia }\end{array}$ & Perpetuating factors & $\begin{array}{c}\text { Yes } \\
\text { - DBAS \& DISRS }\end{array}$ \\
\hline Anxiety about sleep (before sleep \& during the day) & Perpetuating factors & $\begin{array}{l}\text { Yes } \\
\text { - APSQ }\end{array}$ \\
\hline Sleep effort & Perpetuating factors & $\begin{array}{l}\text { Yes } \\
\text { - GSES }\end{array}$ \\
\hline $\begin{array}{l}\text { Conditioned arousal - bed becomes a cue for psycho- } \\
\text { physiological arousal }\end{array}$ & Perpetuating factors & No \\
\hline
\end{tabular}


Note: The table lists variables that may play a role in the pathophysiology and psychopathology of chronic insomnia based on previous (non-TBI) insomnia research. Cognitive and behavioural factors are assumed to be the key insomnia perpetuating factors independently of which medical condition insomnia is comorbid with.

The study assessed factors that maintain insomnia because those can be treatment targets. This approach is not comprehensive but was guided by having a broad perspective but retaining concern about participant fatigue.

TBI = traumatic brain injury; VAS = Visual Analogue Scale; DBAS = Dysfunctional Beliefs and Attitudes about Sleep Scale; DASS 21 = Depression, Anxiety, Stress Scale 21; DISRS= Daytime Insomnia Symptom Response Scale; APSQ = Anxiety and

Preoccupation about Sleep Questionnaire; GSES = Glasgow Sleep Effort Scale 


\section{Rationale for the Present Study}

Given that insomnia symptoms following mTBI have long-term negative prediction of TBI outcomes, it is important to identify individuals who report insomnia symptoms in the early post-injury period, provide follow-up, and conduct diagnostic sleep assessment if clinically significant symptoms do not resolve within the first few post-injury months. To this end, clinical studies that elucidate what sleep variables perpetuate insomnia symptoms and ones that inform assessment tailored to the need of this clinical population are needed.

\section{Objectives and Hypotheses}

The dissertation study had two overarching goals: (1) to determine the prevalence of insomnia disorder, sleep apnea ${ }^{1}$, PLMD and CRSWD among individuals who report new onset/amplified chronic insomnia symptoms following mTBI and (2) to determine which objectively measured electroencephalographic (EEG) and subjective sleep parameters are associated with the perception of poor sleep among patients presenting with chronic insomnia symptoms following a mTBI (for a summary of Objectives and Hypotheses, see Panel 1.6 at the end of this chapter).

A full sleep and circadian assessment has been conducted to achieve the first, clinically orientated main goal. The assessment maps onto two specific objectives:

(1) Objective 1: Determine the percentage of patients with a history of mTBI and current chronic insomnia symptoms who have sleep apnea or PLMD detected by PSG.

(2) Objective 2: Determine both the percentage of abnormal DLMO and the rate of CRSWD in a full sample of individuals reporting symptoms of chronic insomnia following mTBI.

${ }^{1}$ OSA, central and mixed apnea 
The above two objectives cover diagnostic and differential diagnostic issues to inform assessment.

The second main goal is more theoretically oriented and has two specific objectives:

Objective 3: Test the REM instability hypothesis in chronic insomnia following a mTBI (please see below for further elaboration).

Objective 4: Determine which objectively measured EEG and subjective variables are associated with the perception of poor sleep among patients presenting with insomnia symptoms following a mTBI. 
Main Goal 1: Determine the Prevalence of Sleep Disorders that Contribute to Chronic Insomnia Symptoms Following mTBI

\section{A. The use of PSG in the diagnostic assessment of chronic insomnia following mTBI}

A decade-old clinical practice guideline recommends that clinicians employ PSG in insomnia evaluation only when there is an indication that the patient has another sleep disorder that may give rise to insomnia symptoms or may be comorbid with insomnia disorder (SchutteRodin et al., 2008). This, however, does not discount the importance of PSG in differential diagnosis; i.e., detecting sleep disorders that may fully or partially account for the insomnia symptoms. The challenges with conducting PSG only in a few selected cases of insomnia is that the patient may not report symptoms of other sleep disorders that may cause insomnia symptoms, unless asked or prompted - this omission often occurs in non-sleep medical practices. Even when specifically asked, patients may not be aware of symptoms of sleep disorders that lead to insomnia complaints; for example, the specific symptoms of PLMD are often unnoticed by both patients and their bedpartners.

Though PSG is not recommended for routine insomnia diagnosis in general clinical practice, it may be required in specific populations, see Figure 1.2. 


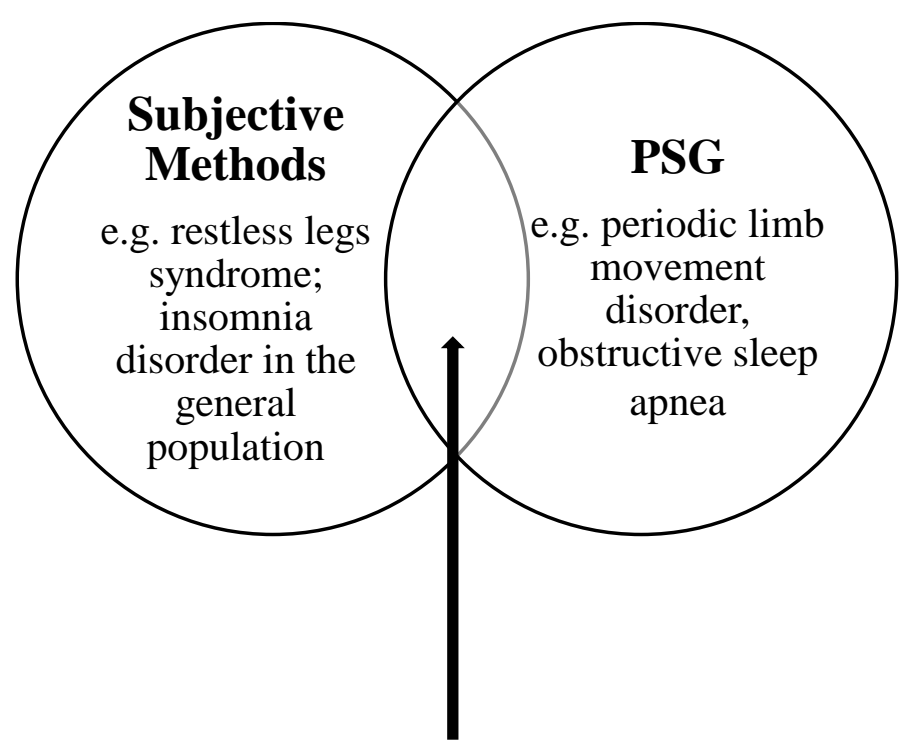

Both subjective tools and PSG are needed in the insomnia workup following TBI?

Figure 1.2. Subjective and PSG Assessment of Sleep Disorders

This figure illustrates that the main diagnostic assessment for certain sleep disorders (e.g. restless legs syndrome and insomnia disorder) is based on subjective methods, while for the diagnosis of other sleep disorders (e.g. OSA and PLMD) PSG is required.

Insomnia assessment is often based solely on subjective assessment tools. However, it is possible that in certain clinical groups the prevalence of sleep disorders that may cause the insomnia symptoms (or be comorbid with insomnia) - e.g. OSA or PLMD - are also common. In these populations, a more deliberate use of PSG would be warranted in insomnia assessment. This may be the case in the insomnia assessment following traumatic brain injury (TBI).

There are no studies involving a homogenous mTBI sample that used insomnia-specific questionnaires, in combination with a clinical interview, sleep diary and PSG to differentiate between insomnia symptoms and insomnia disorder. Consequently, it is unknown what percentage of patients with mTBI and insomnia symptoms (insomnia specific questionnaire scores above cut-off, insomnia symptoms reported in a clinical interview or on a sleep diary) also has sleep apnea or PLMD. This distinction is important with respect to clinical practice. If the prevalence of sleep apnea and PLMD is high, the PSG assessment of these sleep disorders among mTBI patients who present with insomnia symptoms would be warranted. 
Objective 1 and hypothesis 1 . The first aim of this dissertation was to determine the prevalence of patients with a history of mTBI and current chronic insomnia symptoms who have an underlying/comorbid sleep apnea ${ }^{2}$ or PLMD detected by PSG. It was hypothesized that PSG would identify these specific diagnoses (OSA plus PLMD) in the same proportion as in general insomnia samples ( $\geq 30 \%$ ). A secondary goal was to explore the diagnostic utility and acceptability of home PSG in this patient group.

\section{B. Circadian sleep-wake disorders among individuals presenting with symptoms of} chronic insomnia following mild traumatic brain injury. Insomnia is a shared symptom of CRSWD. However, the pathophysiology, assessment and treatment of circadian sleep disorders is different from that of insomnia disorder; therefore, differential diagnosis is crucially important.

In the only study concerning circadian disorders in mTBI, a sub-group of 15 of 42 individuals with insomnia were selected by a sleep specialist for a circadian assessment (Ayalon et al., 2007). These 15 patients were diagnosed with either delayed phase sleep-wake disorder or irregular sleep-wake rhythm disorder. The study had some limitations, including: (1) only onethird of the sample received a circadian assessment and selection criteria were not reported; (2) melatonin was stated to have been assessed, but dim light melatonin onset $\left(\mathrm{DLMO}^{3}\right.$ - the most reliable marker of circadian phase) was not reported; (3) the time since injury was not specified; (4) sleep data of those with and without a circadian sleep disorder were not reported and compared; and (5) age range of the sample was restricted to young and early-middle age adults.

Objective 2 and hypothesis 2 . The second objective was to replicate and extend the previous research by determining both the percentage of abnormal DLMO and the prevalence of CRSWD

\footnotetext{
${ }^{2}$ OSA, central or mixed apnea

${ }^{3}$ Endogenous melatonin is released in the circadian evening. DLMO is the clock time when melatonin concentration reaches a standard threshold; for further details please see Appendix D and Appendix E.
} 
in a full sample of individuals reporting symptoms of chronic insomnia following mTBI. Based on previous research, the hypothesis was that rate of delayed DLMO and CRSWD would be higher in the mTBI sample than the known prevalence in a relevant comparison group - i.e. in individuals who seek treatment for chronic insomnia in sleep clinics (10\%) (AASM, 2008).

Main Goal 2: Determine Which Objectively Measured EEG and Subjective Factors are Associated with the Perception of Poor Sleep among Patients Presenting with Insomnia Symptoms following a mTBI. Patients often seek help for insomnia if they feel that there is "something wrong" with their sleep based on their perception of being awake at night, having low sleep quality or not feeling refreshed from sleep. It is possible that perceived time spent awake during the night, low sleep quality and subjective ratings of feeling unrefreshed after sleep are associated with objectively detected sleep parameters, for example the percentage of different sleep stages, short awakenings from sleep, or EEG signs of arousal. Another possibility is that subjective ratings of sleep quality and being unrested have stronger relations with subjective variables, for example people's perception of time spent awake during the night, the pain or fatigue they experience before they go to bed, or with their general mood and anxiety.

\section{A. Testing the REM instability hypothesis in chronic insomnia following a mTBI. The REM} sleep instability hypothesis of insomnia posits that worry about sleep leads to short awakenings (i.e., micro arousals) from sleep and, in particular, from REM sleep (Riemann et al., 2012). During micro-awakenings from REM sleep, the individual becomes cognizant of anxious dream content, which resembles daytime and evening sleep-focused anxious thoughts. Consequently, REM sleep fragmentation blurs the distinction between sleep and wakefulness and causes a false perception of being awake while the individual is, in fact, asleep. 
The REM sleep instability hypothesis ${ }^{4}$ is based on a study in which the frequency of micro-arousals was observed to be significantly higher in a group of 100 medication-free individuals with primary insomnia as compared to a control group of 100 good sleepers (Feige et al., 2008). Furthermore, subjective wake time during the night was predicted by both objective PSG wake time and the time spent in REM sleep. The authors' interpretation of this finding was that individuals with insomnia perceived and remembered REM sleep as time spent awake. Interestingly, the number of arousals was not a predictor of subjective wake time above and beyond REM sleep duration in the above study. If the REM instability hypothesis is correct, there may be a variable - other than the number or rate of micro-arousals- that corresponds with a (mis)perception of being awake while the individual is in REM sleep.

Sleep and wakefulness represent different levels of consciousness. One can speculate that patients with insomnia disorder have a heightened level of consciousness and increased awareness of the dream which reflects daytime thoughts and experiences. This could contribute to the misperception of REM sleep as wakefulness.

Objective 3 and hypothesis 3. The third objective of the dissertation study was to test and extend the REM instability hypothesis in patients with mTBI. Based on previous results in primary insomnia, the third dissertation hypothesis was that wake time according to sleep diary (sleep onset latency plus wake after sleep onset) would be significantly correlated with PSG wake time (sleep onset latency plus wake after sleep onset) and with time spent in REM. As an extension of the theory, it was also proposed that subjective wake time would be associated with the relative level of consciousness during REM sleep.

\footnotetext{
${ }^{4}$ The authors did not define the concept "REM instability" but presumably it refers to REM sleep disruption by micro-arousals. The current study measured micro-arousal rate in REM sleep (see Chapter 6) but the dissertation uses the term "REM instability" only to refer to the original name of the theory (described shortly here and in more detail in Chapter 6).
} 


\section{B. Correlates of sleep quality and the feeling of being rested in the morning among patients}

with chronic insomnia symptoms following a mTBI. Subjective low sleep quality and not feeling refreshed from sleep are common but poorly understood complaints of individuals with insomnia disorder (AASM, 2014). Given that our understanding is limited of these common subjective experiences of patients with insomnia disorder, an exploratory approach to investigate these constructs is merited.

Previous research is limited by: (1) reliance of questionnaires in assessing sleep quality and feeling restored from sleep and (2) exclusive focus on "primary insomnia" in studies attempting to determine the EEG correlates of subjective sleep perception. This study extends previous research by exploring the association of daily, prospective VAS measures of sleep quality and feeling rested in the morning with routinely measured sleep EEG parameters, subjective sleep parameters, mood, anxiety, pain and fatigue among individuals who suffer from persistent insomnia symptoms following a mTBI.

Objective 4 and hypothesis 4 . The last objective of the present study was to explore the relationship between subjective sleep diary measures (i.e. sleep onset latency, wake after sleep onset, total wake time, total sleep time); macro and micro EEG parameters (i.e. sleep onset latency, wake after sleep onset, total wake time, total sleep time, slow wave sleep duration, and micro-arousal rate); as well as subjective measures of pain, fatigue, anxiety, and mood, as predictors of perceived sleep quality and ratings of feeling rested in the morning. The hypothesis was that subjective total wake time would have the highest correlation with the outcome measures. 
Panel 1.6. Objectives and Hypotheses

Main Goal 1: Determine the prevalence of sleep disorders (insomnia disorder, sleep apnea, PLMD, CRSWD) that contribute to chronic insomnia symptoms following a mTBI.

Objective 1. Determine the prevalence of patients with a history of mTBI and current chronic insomnia who have sleep apnea or PLMD detected by PSG.

Hypothesis 1. PSG would identify specific diagnoses in the same proportion as in general insomnia samples $(\geq 30 \%)$. A secondary goal was to explore the diagnostic utility and acceptability of home PSG in this patient group.

Objective 2. Replicate and extend the previous research by determining both the percentage of abnormal DLMO and the prevalence of CRSWD in a full sample of individuals reporting symptoms of chronic insomnia following mTBI.

Hypothesis 2. Based on previous research, the hypothesis was that rate of delayed DLMO and CRSWD would be higher in the mTBI sample than is reported for individuals who seek treatment for chronic insomnia in sleep clinics (10\%) (AASM, 2008).

Main Goal 2: Determine which objectively measured EEG and subjective variables are associated with the perception of poor sleep among patients with chronic insomnia symptoms following a mTBI.

Objective 3. Test and extend the REM instability hypothesis in patients with mTBI.

Hypothesis 3. Based on previous results in primary insomnia, the hypothesis was that wake time according to sleep diary (sleep onset latency plus wake after sleep onset) would be significantly correlated with PSG wake time (sleep onset latency plus wake after sleep onset) and with time spent in REM. As an extension of the theory, it was also proposed that subjective wake time would be associated with the relative level of consciousness during REM sleep.

Objective 4. Explore the relationship between subjective sleep diary measures (i.e. sleep onset latency, wake after sleep onset, total wake time, total sleep time); macro and micro EEG parameters (i.e. sleep onset latency, wake after sleep onset, total wake time, total sleep time, slow wave sleep duration, and micro-arousal rate); as well as subjective measures of pain, fatigue, anxiety, and mood, as predictors of perceived sleep quality and ratings of feeling rested in the morning.

Hypothesis 4. Subjective total wake time would have the highest correlation with the outcome measures. 


\section{Chapter 2: Methods}

\section{Participant Characteristics}

Individuals reporting symptoms of chronic insomnia following a mTBI and seen at a hospital or community clinics were referred to participate in this study. Potential participants were referred from health care settings that provide mTBI assessment and management, including head injury clinics $(36 \%)$, family medicine practices $(32 \%)$, concussion clinics and sport medicine clinics (12\%), practices of speech language pathologists, neurologists, physiatrists, and physiotherapists (8\%) and emergency medicine specialists (4\%) in Ontario, Canada. An additional $8 \%$ of the referrals were directed to the study from other sleep clinics. Inclusion criteria were: (a) mTBI (Glasgow coma score of 13 or higher) three to 24 months prior to the screening assessment; (b) chronic insomnia symptoms that started after or were present prior to the injury but significantly amplified following the injury; (c) age between 16 and 65 years; (d) could read and speak English without the use of a translator; and (e) able to provide informed consent. The lower threshold for including participants in the study was three months post-injury because insomnia symptoms must persist for at least three months to meet diagnostic criteria for insomnia disorder. The 24 months was a somewhat arbitrary upper limit to include individuals who sustained a TBI in a relatively recent past.

Excluded were those who: (a) had alcohol or other substance use problems within three months prior to enrollment based on DSM 5 criteria (APA, 2015); (b) needed immediate psychiatric help based on in-person assessment (for example imminent and high suicidal risk or acute psychosis); (c) had pre-existing brain disorders (e.g. brain tumor, neurodegenerative disorders, infections) or had neurosurgery; or (d) had travelled across more than two time zones less than three weeks prior to the phone screening. According to the protocol, shift workers on 
night or rotating shift schedule were to be excluded from the melatonin assessment but none of the individuals referred to the study had such work schedule.

Eighty-one persons completed the phone screening and 62 of them participated in the inperson clinical interview. Reasons for not participating in the clinical interview were: (a) not being eligible to participate in the study (10 persons); (b) scheduling conflicts (three persons); (c) booking a sleep assessment at another clinic closer to residence (two persons). Others did not come for the interview for unknown reasons. Based on the interview results, 12 individuals were excluded from the study (11 did not endorse symptoms of chronic insomnia and one had substance use disorder). The final sample consisted of 50 participants, which was the required sample size based on a priori calculations.

\section{Sample Size Calculation}

The sample size was geared towards the first two hypotheses - based on previous research it was anticipated that if these hypotheses were confirmed, approximately 15-25 participants would have sleep apnea, PLMD or CRSWD in the sample of 50 (Ayalon et al, 2007; Mathias \& Alvaro, 2012). With the sample size required to test the first two hypotheses, the study was also powered to detect medium correlations (correlation coefficient is at least .40) for the third and fourth hypotheses with $\alpha=.05$ and power $=.80$ based on a $\mathrm{G}$ Power calculation.

\section{Procedures}

Study procedures are outlined in Table 2.1 and Figure 2.1.

\section{Apparatus and Measures}

Assessment tools are listed below in Table 2.1 and described in detail in Appendix D. 
Table 2.1.

Study Procedures

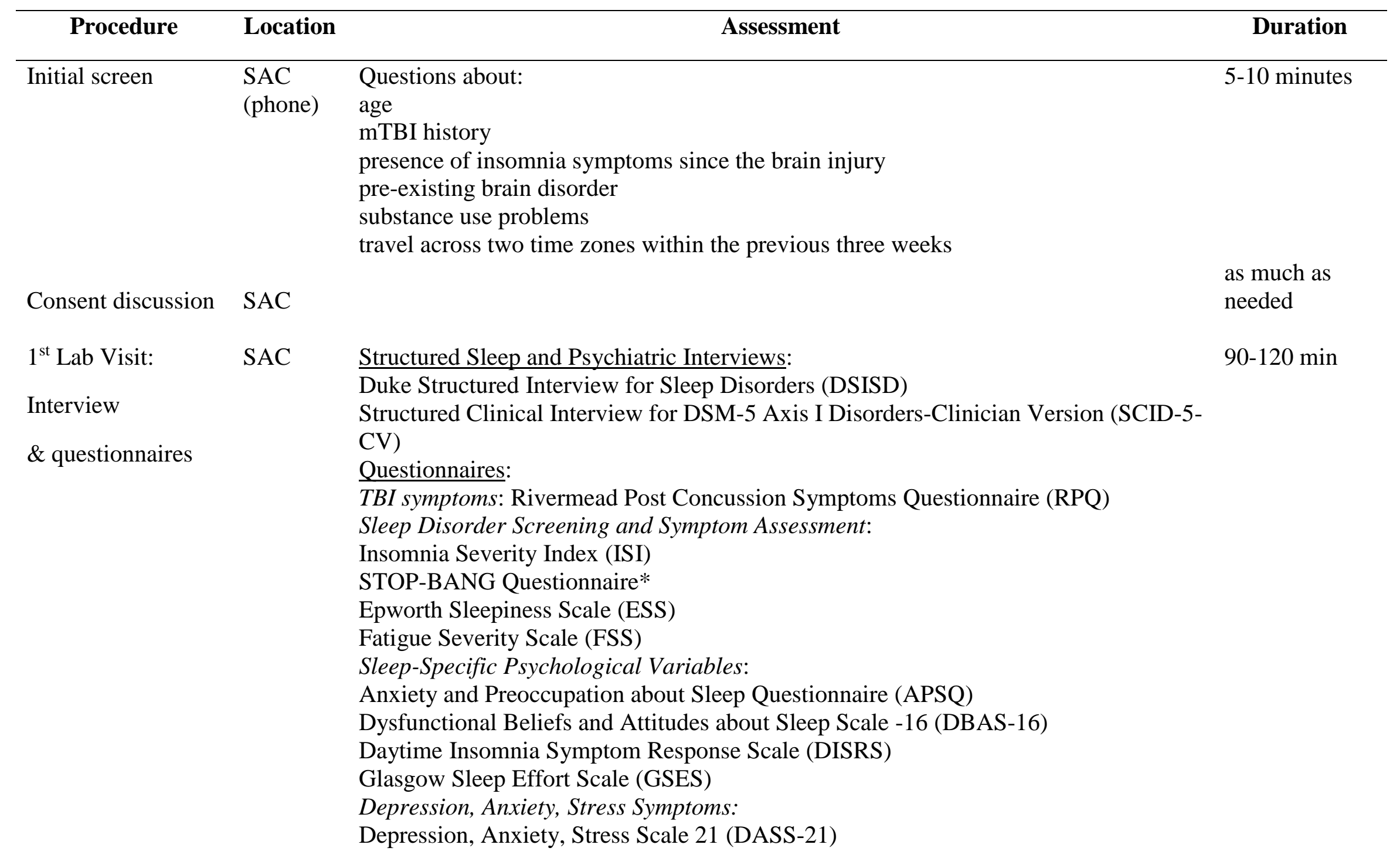




\begin{tabular}{|c|c|c|c|}
\hline Procedure & Location & Assessment & \\
\hline $\begin{array}{l}\text { Prospective Sleep } \\
\text { and Fatigue } \\
\text { Monitoring }\end{array}$ & Home & $\begin{array}{l}\text { Actigraphy (continuous for } 2 \text { weeks) } \\
\text { Sleep diary completed in the mornings (approx. } 5 \text { minutes) } \\
\text { Daily VAS sleep quality and restorative sleep ratings (approx. } 1 \text { minute/VAS) } \\
\text { Daily VAS fatigue and pain ratings (approx. } 1 \text { minute/VAS) }\end{array}$ & 2 weeks \\
\hline $\begin{array}{l}\text { Home PSG } \\
\text { recording }\end{array}$ & Home & $\begin{array}{l}\text { Two consecutive nights of PSG with NeuroZone MSH home recording system 6-7 } \\
\text { days following the lab PSG }\end{array}$ & $\begin{array}{l}\text { Normal sleep } \\
\text { duration plus } \\
\text { approximately } \\
30 \text { minutes set- } \\
\text { up time }\end{array}$ \\
\hline $3^{\text {rd }}$ Lab Visit: & $\begin{array}{l}\text { SAC } \\
\text { MET }\end{array}$ & Dim Light Melatonin Onset (DLMO) test & 7.5 hours \\
\hline $\begin{array}{l}\text { Return actiwatch, } \\
\text { PSG device, \& } \\
\text { sleep log; } \\
\text { complete } \\
\text { questionnaires; } \\
\text { DLMO test }\end{array}$ & & $\begin{array}{l}\text { Questionnaires: } \\
\text { Morningness-Eveningness Questionnaire } \\
\text { Assessment Acceptability Questionnaire }\end{array}$ & \\
\hline
\end{tabular}

Note. SAC = Sleep and Alertness Clinic; TBI = traumatic brain injury; PSG = Polysomnography; VAS = Visual Analogue Scale, MET $=$ Melatonin Testing Lab * STOP-BANG scores were calculated after BMI and neck circumference measurement.

Participants received $\$ 150$ to cover parking and as for their time. 
Visit 1: Interview \& questionnaires

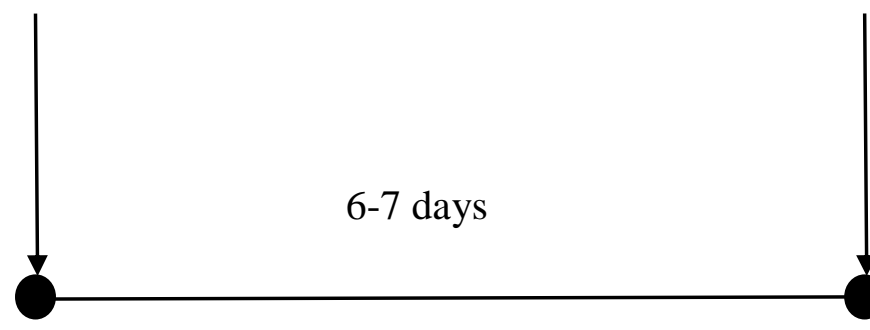

actigraphy, daily sleep diary \& VAS
Visit 2: Lab PSG

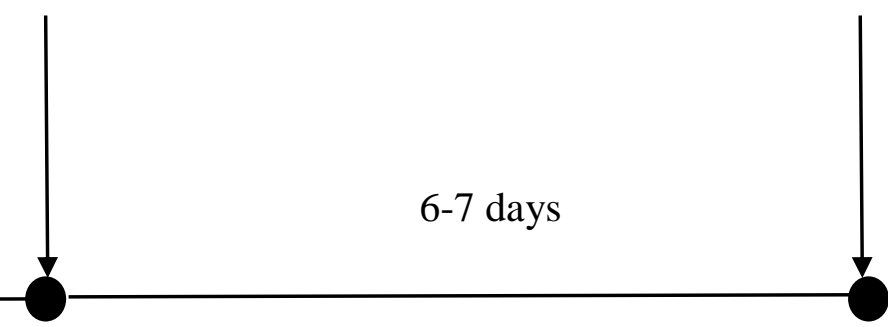

2 nights of home PSG, actigraphy, daily sleep diary \& VAS

\section{Figure 2.1. Study procedures}

This figure shows that participants visited the sleep lab three times during the 12-14 days of the study. They also wore a wrist actiwatch, completed a standard sleep diary daily and used VAS to indicate their level of fatigue and pain (evenings) and rate in their sleep quality and the extent to which they felt rested/refreshed (mornings). Additionally, they conducted two nights PSG recordings at home during the second week of the study period.

Hypnotic medications were discontinued before data collection commenced. Norepinephrine reuptake inhibitors as well as alpha and beta-adrenergic medications were discontinued before the DLMO test. 


\section{Chapter 3: Description of Participant Characteristics, Questionnaire Scores, Sleep Diary and Actigraphy Sleep Indices}

Participants' characteristics, questionnaire score distributions as well as the summary of sleep diary and actigraphy sleep indices are described in this section. These results are summarized at the end of this chapter and the key points are discussed in Chapter 8 . The results pertaining to the four study objectives are presented in Chapters 4-7.

\section{Analyses of Participant Characteristics, Questionnaire Scores, Sleep Diary and Actigraphy Sleep Indices}

Descriptive statistics were used to summarize participant characteristics, questionnaire score distributions as well as sleep diary and actigraphy sleep indices. Bivariate correlation analyses determined the strength of relationship between questionnaire scores. Non-parametric, tests (Kendell's tau) were conducted to quantify the correlation between non-normally distributed actigraphy and sleep diary indices. ANOVAs compared the mean scores of sleep specific psychological variables across sleep disorder diagnostic categories. Chi square tests compared the percentage of patients with excessive daytime sleepiness across diagnostic groups. Bootstrapping was used to calculate the $95 \%$ bias-corrected accelerated confidence intervals of the point estimates (means, medians and correlation coefficients) based on 2000 resampling. Specific analyses related to the study objectives and hypotheses are described in Chapters 4 -7.

\section{Missing Data}

The percentage of missing data per study procedure is summarized in Table 3.1 below. Missing data were excluded pairwise in statistical analyses. 
Table 3.1.

Missing Data

Assessment

Missing (percent of the sample)

Clinical interview

0

Visit 1 Questionnaire booklet

Sleep Diary

12

VAS

12

Actigraphy

4

Lab PSG

0

Home PSG

one night missing: 23 two nights missing: 5

DLMO

6

Visit 3 Questionnaires

MEQ: 18 AAQ: 30

Note. VAS = visual analogue scales; $\mathrm{PSG}=$ polysomnography; $\mathrm{DLMO}=$ dim light melatonin onset test; MEQ = Morningness-Eveningness Questionnaire; AAQ = Assessment Acceptability Questionnaire. The MEQ was added to the protocol approximately two months following the commencement of data collection and therefore it is not available from the first group of participants.

\section{Participants}

Participant characteristics are summarized in Table 3.2 below. 
Table 3.2.

Participant Characteristics (Categorical Demographic Variables)

$$
\mathrm{n}(\%)
$$

\section{Gender}

Male

$18(36)$

Female

$32(64)$

\section{Education}

High school

$13(26)$

College

$12(24)$

University

$25(50)$

\section{Employment status}

Full time

$21(42)$

Part time

$5(10)$

Student

Unemployed

8 (16)

Sick leave/disability

$11(22)$

Retired

$1(2)$

\section{Living arrangement}

With family/partner

37 (74)

With roommates

Alone

9 (18) 
Table 3.2.

Participant Characteristics (mTBI History)

$\mathrm{n}(\%)$

\begin{tabular}{lr}
\hline Number of concussions* & \\
Single & $32(64)$ \\
Multiple & $18(36)$ \\
Two & $7(14)$ \\
Three & $3(6)$ \\
Four & $3(6)$ \\
Five & $2(4)$ \\
Exact number & $3(6)$ \\
unknown &
\end{tabular}

\section{Cause of injury}

Car accident

Sport injury

Fall

Object hit the head

Physical assault

Workplace injury
$18(36)$

$18(36)$

9 (18)

$2(4)$

$2(4)$

$1(2)$

Note: * Based on self-report. If a participant had multiple concussions, it is the the injury that preceded the onset of the sleep problem 
Table 3.2.

Participant Characteristics (Medical History)

$$
\mathrm{n}(\%)
$$

\section{Participants having comorbid medical conditions}

$22(44)$

Chronic pain

Hypertension

Gastrointestinal disease (e.g. GI reflux)

Hyperlipidemia

Asthma

Autoimmune disease

Hypothyroidisms*

$2(4)$

Osteoporosis

Heart disease

Participants reporting past psychiatric conditions

$23(46)$

Current psychiatric conditions

$13(26)$

Major Depressive Disorder

Social Anxiety Disorder

Panic Disorder

Obsessive Compulsive Disorder

Specific Phobia

Participants used sleep interventions (lifetime)*

\section{Participants sought medical help for sleep} (lifetime)*

Note: Some participants had multiple comorbidities; therefore, the sum of percentages is higher than $100 \%$. * participants reporting a history of hypothyroidism were on thyroid supplements *data was not available from the full sample 
In brief, age range was 17-62 (median age: 39.5), approximately two-thirds of the sample was female; worked full time, part time or studied as a student; and had a single mTBI from various causes. Approximately three-quarters of the sample completed higher education (college or university) and lived with their family. Participants were enrolled in this study on average 13 months (SD: 7.42) following their head injury. Three quarters of the sample denied having subjective sleep problems or sleep disorders prior to their injury and $100 \%$ reported moderate or significant worsening of their sleep since the injury. The most common causes of the injury were motor vehicle accidents and sport injuries (36\% each), followed by falls (18\%) and other causes (physical assault, object hit the head and workplace injury). Close to half of the sample reported a history of psychiatric conditions and one-quarter reported symptoms that met criteria for current mental health conditions, predominantly major depressive disorder, see Table 3.2 on the previous page. Apart from the insomnia symptoms, the mTBI was the only medical condition in $56 \%$ of the sample, while $44 \%$ had comorbidities; the most common ones were chronic pain (26\%), hypertension (10\%), gastrointestinal diseases (10\%) as well as hyperlipidemia and asthma (6\% each), see Table 3.2.

\section{Questionnaire Scores}

The descriptive statistics of the questionnaires are summarized in Table 3.3. 
Table 3.3.

Descriptive Characteristics of the Study Questionnaires

\begin{tabular}{|c|c|c|c|c|c|c|c|}
\hline Variables & Mean & $\begin{array}{c}95 \% \text { bias } \\
\text { corrected CI }\end{array}$ & Median & $\mathrm{SD}$ & Minimum & Maximum & $\begin{array}{c}\text { Interquartile } \\
\text { range }\end{array}$ \\
\hline FSS & 5.49 & $5.12-5.77$ & 5.85 & 1.11 & 1.00 & 7.00 & 1.35 \\
\hline ESS* & 10.42 & $9.04-11.74$ & 9.5 & 5.22 & 0.00 & 21 & 8 \\
\hline ISI* & 20.08 & $19.08-21.10$ & 20.00 & 3.85 & 11 & 28 & 5 \\
\hline DASS-21 Stress & 17.56 & $14.73-20.39$ & 16 & 9.96 & 0 & 36 & 16 \\
\hline DBAS* & 6.28 & $5.89-6.66$ & 6.37 & 1.42 & 2.94 & 9.06 & 2.2 \\
\hline DISRS* & 52.79 & $49.69-55.77$ & 54 & 11.18 & 28 & 77 & 12 \\
\hline
\end{tabular}

Note: $\quad$ FSS = Fatigue Severity Scale; ESS = Epworth Sleepiness Scale; ISI = Insomnia Severity Index; DASS-21= Depression Anxiety Stress Scale -21; DBAS = Dysfunctional Beliefs and Attitudes Scale; DISRS = Daytime Insomnia Symptom Response Scale; APQS = Anxiety and Preoccupation about Sleep Questionnaire; GSES = Glasgow Sleep Effort Scale; MEQ = MorningnessEveningness Questionnaire. 95\% bias corrected CI = 95\% bias corrected confidence interval of the mean based on 1000 bootstrap Samples. The star sign denotes normally distributed questionnaire scores. 


\section{Questionnaire assessing TBI symptoms.}

The Rivermead Post-Concussion Symptom Questionnaire (RPQ) assesses 16 symptoms that patients commonly report after concussion (RPQ; King et al., 1995). Participants rated the extent to which they endorsed each symptom (as compared to before the accident). In this study, items that garnered the highest scores were sleep and fatigue (median scores $=4$ ). Participants also reported that headache and cognition (i.e. forgetfulness, poor concentration, difficulty with thinking) were moderate problems since the injury (median scores $=3$ ). The scores on the noise and light sensitivity as well as on the irritability items were in the mild to moderate range (mean =2.5). Participants rated the remaining items as being mild problems or not a problem.

\section{Sleep disorder screening and symptom questionnaires.}

The Insomnia Severity Index (ISI) measures the subjective severity of insomnia symptoms and has been recommended by an expert panel for use in insomnia research (Buysse, Ancoli-Israel, Edinger, Lichstein, \& Morin, 2006; Morin, 1993a). The mean ISI score in this sample was in the range of "moderate" insomnia (Morin, 1993b). Each participant scored above the recommended threshold for screening clinical samples (ISI $\geq 11$ ) and $92 \%$ had scores indicating the presence of clinically significant, moderate or severe insomnia symptoms at the time of the clinical interview, see Table 3.4 below.

Table 3.4.

Insomnia Severity Categories Based on ISI Scores

\begin{tabular}{lc}
\hline & n (\%) \\
\hline None (score 0-7) & $0(0)$ \\
Sub threshold (score 8-14) & $4(8)$ \\
Moderate (score 15-21) & $24(48)$ \\
Severe (score 22-28) & $22(44)$ \\
\hline
\end{tabular}


Note. Severity of insomnia in the sample based on recommended cut-off scores (Bastien, Vallieres, \& Morin, 2001). ISI = Insomnia Severity Index

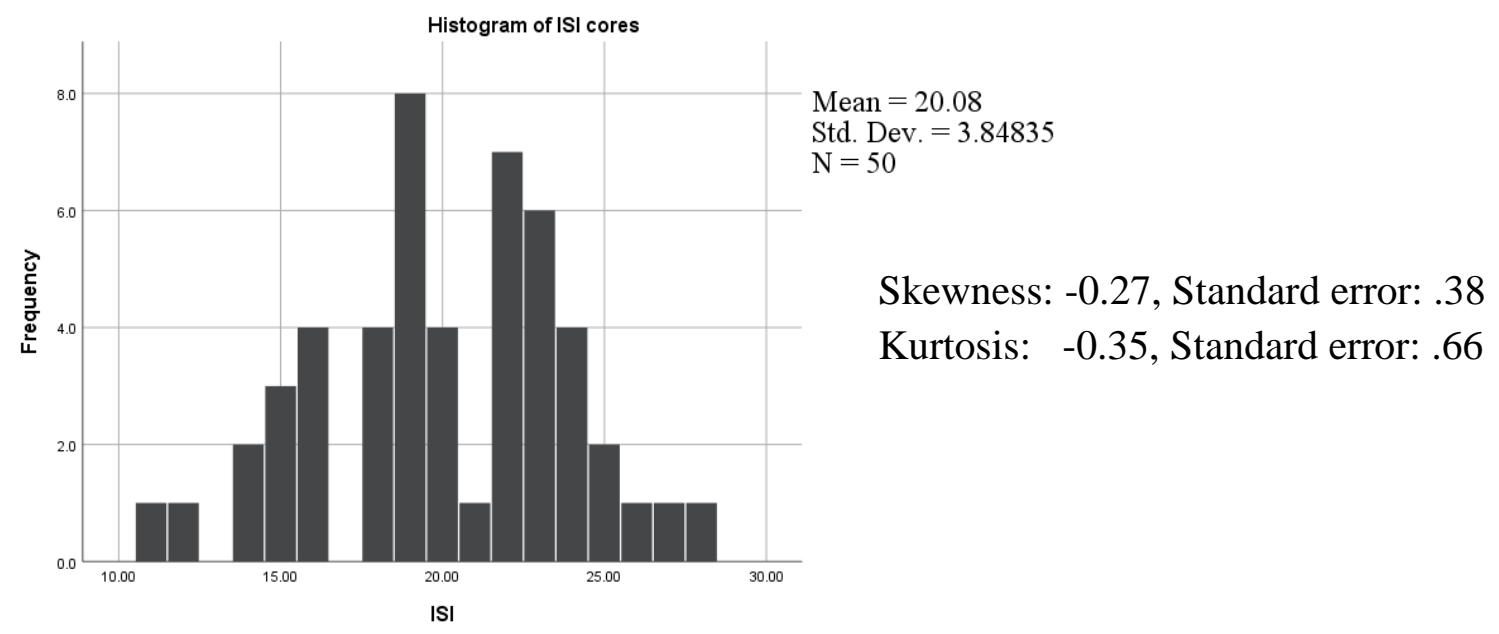

Figure 3.1. Insomnia Severity Index (ISI) Score Distribution

The Fatigue Severity Scale (FSS) measures fatigue-related functional impairment (Krupp, LaRocca, Muir-Nash, \& Steinberg, 1989). The full sample - with the exception of only three participants - obtained a score indicating clinically significant, moderate or severe fatigue related functional impairment $(\mathrm{FSS} \geq 4)$.

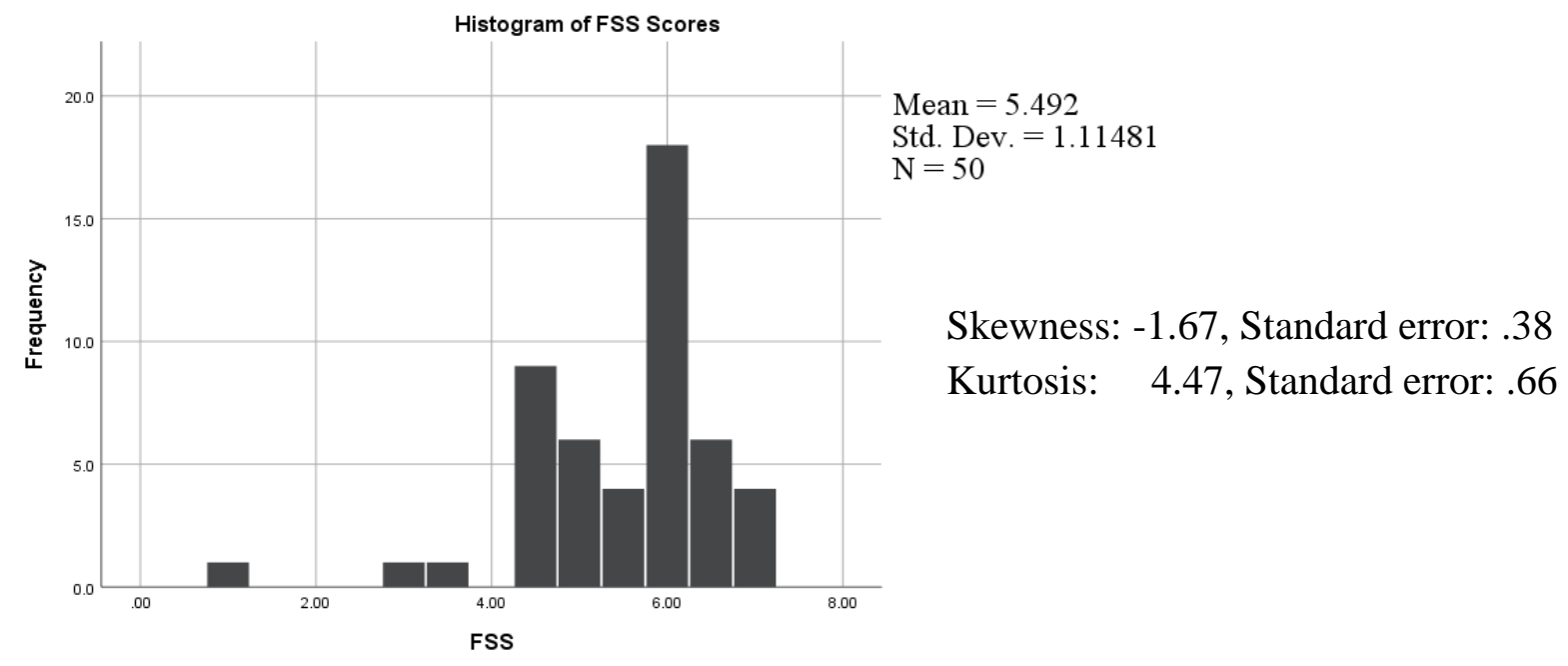

Figure 3.2. Fatigue Severity Scale (FSS) Score Distribution 
The Epworth Sleepiness Scale (ESS; Johns 1991) measures subjective excessive daytime sleepiness. The ESS lists eight life situations and asks respondents to mark the likelihood to which they would fall asleep in the given situation. Scores range from 0 to 24 . A score above 10 indicates excessive subjective daytime sleepiness. Almost half (48\%) of this sample reported symptoms of excessive subjective daytime sleepiness. Two - thirds of patients with insomnia disorder, half of the group with OSA/PLMD and one-third of patients with a CRSWD had excessive daytime sleepiness; these percentages are not statistically different across the above diagnostic categories $\chi^{2}=5.36, p=.68$

The STOP-BANG questionnaire is a screening tool for OSA. The scores range between zero and eight and higher score indicates a higher likelihood that someone has OSA. The Questionnaire scores had a restricted range (0-5) in this study; see Figure 3.3 (Chung et al., 2008). Seventy nine percent of this sample scored below the cut-off score three, recommended to rule out OSA cases in sleep clinic settings. This means that $79 \%$ of participants would not have received further OSA assessment based on these screening results. The predictive value of different OSA cut-off scores in this study suggests that a lower cut-off may be needed to rule out OSA in post mTBI chronic insomnia - this is discussed in Chapter 4. 


\section{STOP-BANG scores}

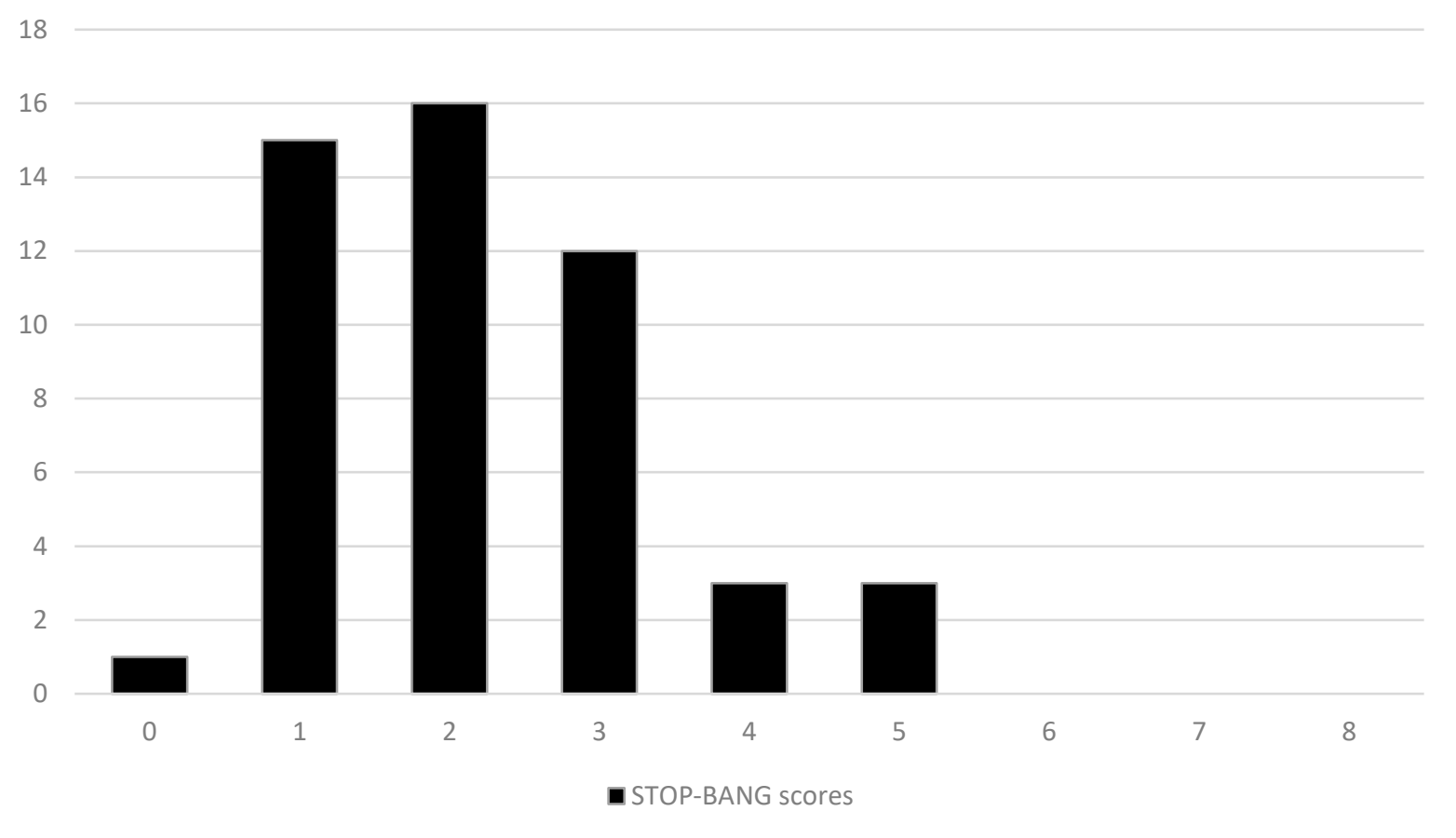

Figure 3.3. STOP-BANG Questionnaire Score Distribution

The figure shows the distribution of the STOP-BANG questionnaire scores in the sample. The horizontal axis shows the questionnaire scores and the vertical axis shows the number of participants. The questionnaire scores range from zero to eight. The minimum score was zero and the maximum score was 5 in this sample. The recommended cut-off to rule out OSA is 3 and the cut-off for moderate/severe OSA is 5 in sleep clinics.

\section{Depression, anxiety and stress symptoms.}

Depression Anxiety Stress Scale -21 (DASS -21) is a 21-item questionnaire assessing symptoms of depression, anxiety, and stress (Lovibond \& Lovibond, 1995). Specifically, the depression subscale contains items that refer to low mood, the anxiety subscale assesses symptoms of psychophysiological arousal, and the stress subscale captures the signs of overlapping tension/irritability that are components of both depression and anxiety (Antony, Bieling, Cox, Enns, \& Swinson, 1998). In this study, depression, anxiety and stress scores ranged 
from normal to extremely severe, see Table 3.5. It is important to note that these scores do not map directly into psychiatric diagnostic categories.

Table 3.5.

Distribution of DASS Scores

\begin{tabular}{lccc}
\hline & $\begin{array}{c}\text { Depression } \\
\mathrm{n}(\%)\end{array}$ & $\begin{array}{c}\text { Anxiety } \\
\mathrm{n}(\%)\end{array}$ & $\begin{array}{c}\text { Stress } \\
\mathrm{n}(\%)\end{array}$ \\
\hline Normal & $18(32)$ & $19(38)$ & $23(46)$ \\
Mild & $4(8)$ & $6(12)$ & $7(14)$ \\
Moderate & $15(30)$ & $10(20)$ & $5(10)$ \\
Severe & $4(8)$ & $7(14)$ & $10(20)$ \\
Extremely severe & $9(18)$ & $8(16)$ & $5(10)$ \\
\hline
\end{tabular}

Note. DASS = Depression, Anxiety, Stress Scale. The severity ratings are based on the recommended cut off scores (Lovibond \& Lovibond, 1995). Depression: normal (0-9), mild (1013), moderate (14-20), severe (21-27), extremely severe (28+). Anxiety: normal (0-7), mild (89 ), moderate (10-14), severe (15-19), extremely severe (20+). Stress: normal (0-14), mild (1518), moderate (19-25), severe (26-33), extremely severe (37+). 


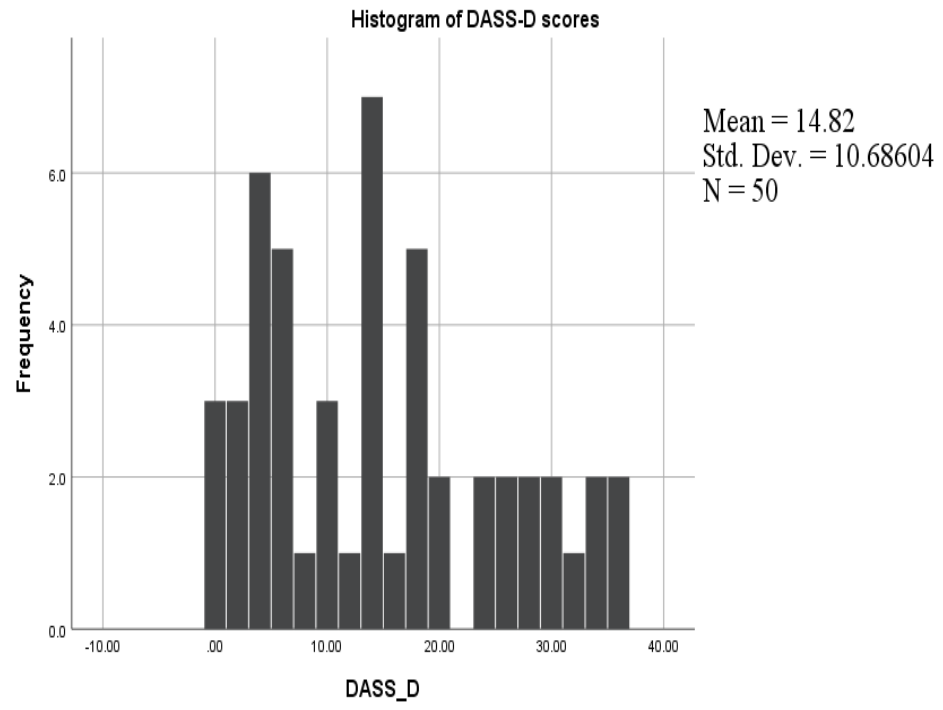

Skewness: -0.49, Standard error: .38

Kurtosis: -0.91 , Standard error: .66

Figure 3.4. Depression Anxiety Stress Scale - Depression Subscale (DASS-D) score distribution

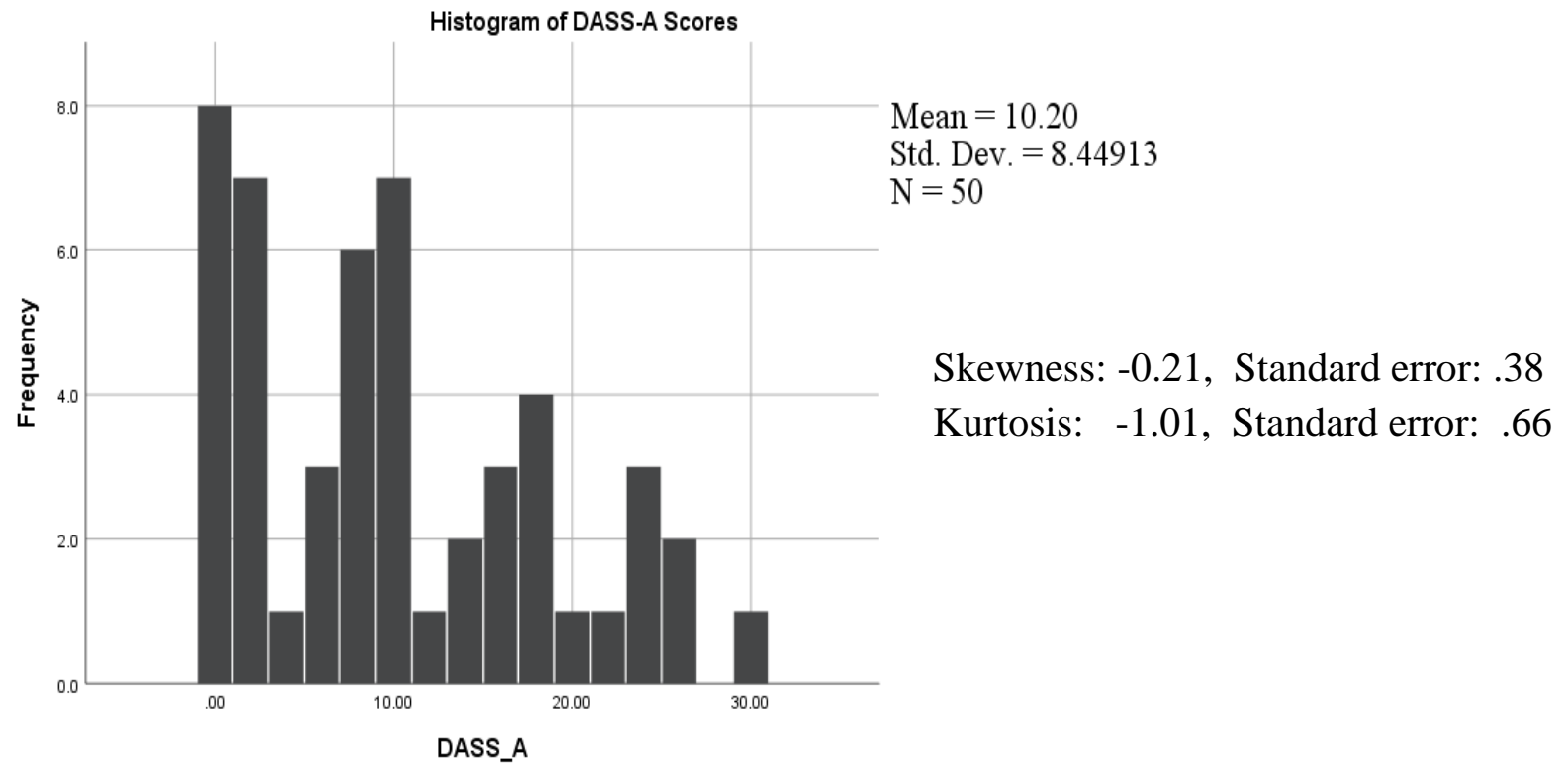

Figure 3.5. Depression Anxiety Stress Scale - Stress Subscale DASS-A score distribution 


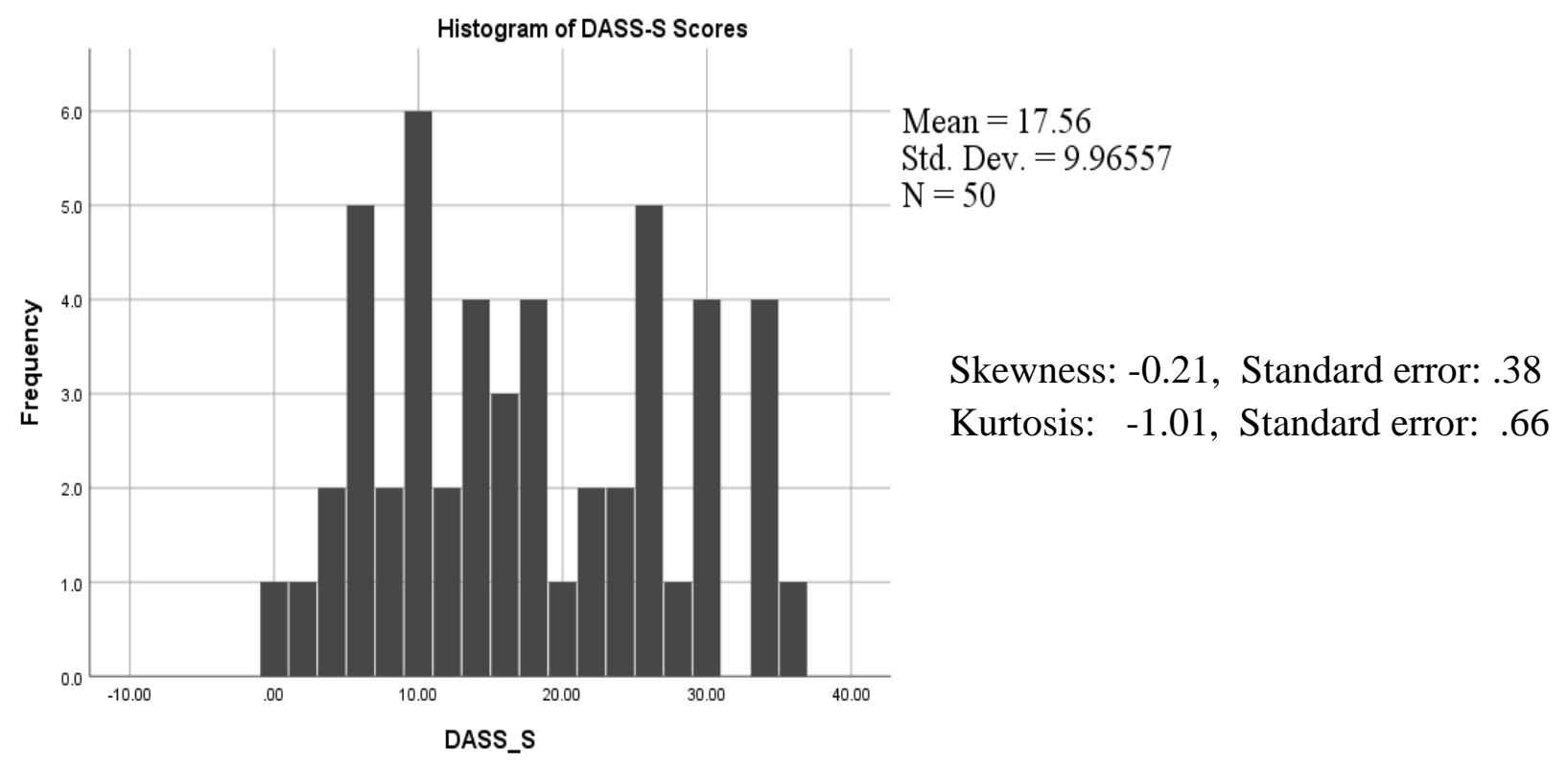

Figure 3.6. Depression Anxiety Stress Scale - Stress Subscale DASS-S score distribution

\section{Sleep-specific psychological variables.}

The Dysfunctional Beliefs and Attitudes Scale (DBAS-16) assesses beliefs and attitudes that maintain chronic insomnia (Morin, 1994; Morin, Vallieres, \& Ivers, 2007). The mean score in the current study (6.28) was higher than the mean score in the validation insomnia sample (Morin, 1994). 


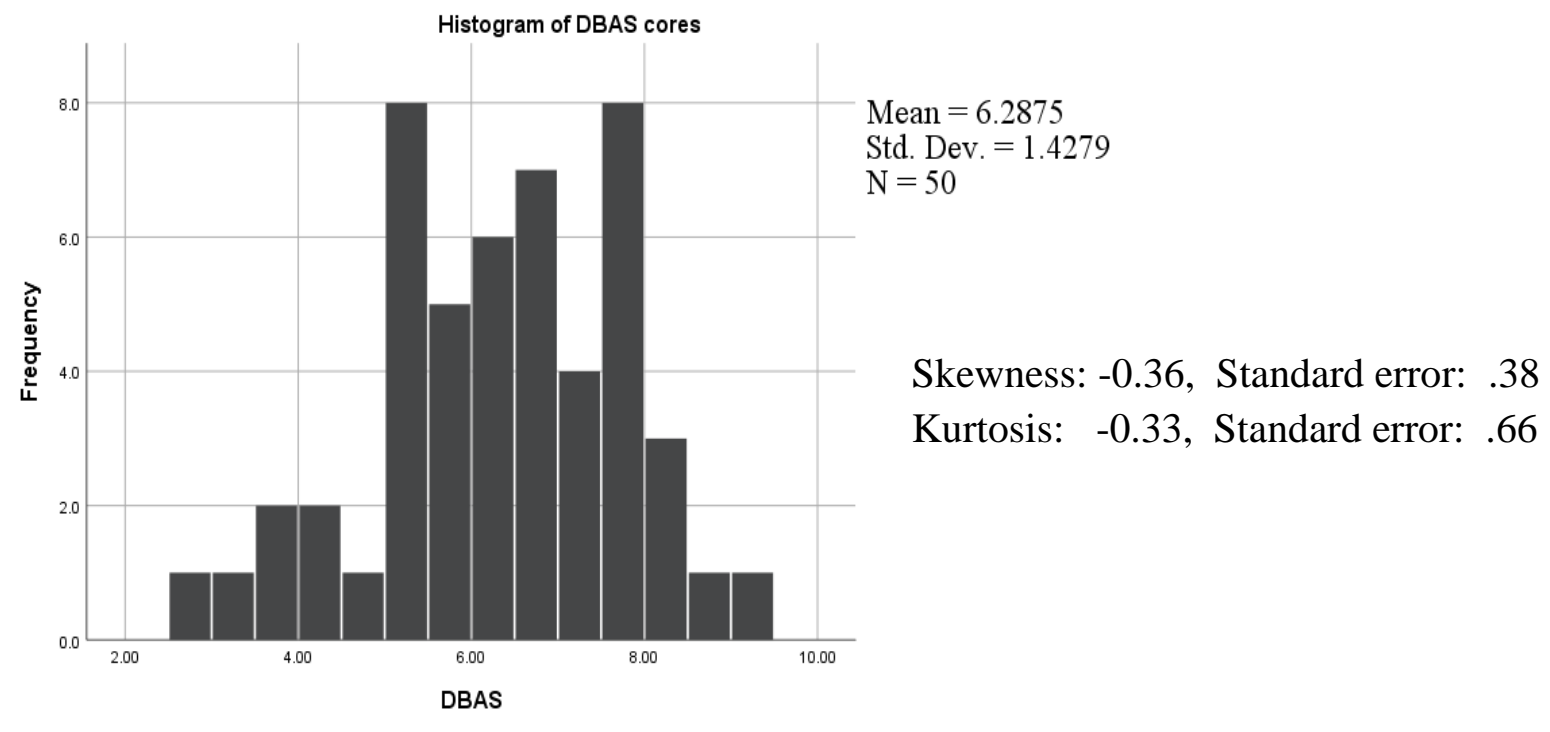

Figure 3.7. Dysfunctional Beliefs and Attitudes Scale (DBAS -16) score distribution

The Daytime Insomnia Symptom Response Scale (DISRS) assesses rumination about the daytime symptoms of insomnia, including decreased motivation, low mood, and tiredness (Carney, Harris, Falco, \& Edinger, 2013). The mean score in this sample $(52.79, S D=11.18)$ was higher than the mean score of the non-clinical validation sample $(41.43, S D=11.42)$ but was similar to the mean score of the clinical (insomnia and depression) validation sample 56.20 $(S D=11.60)$ (Carney et al., 2013). 


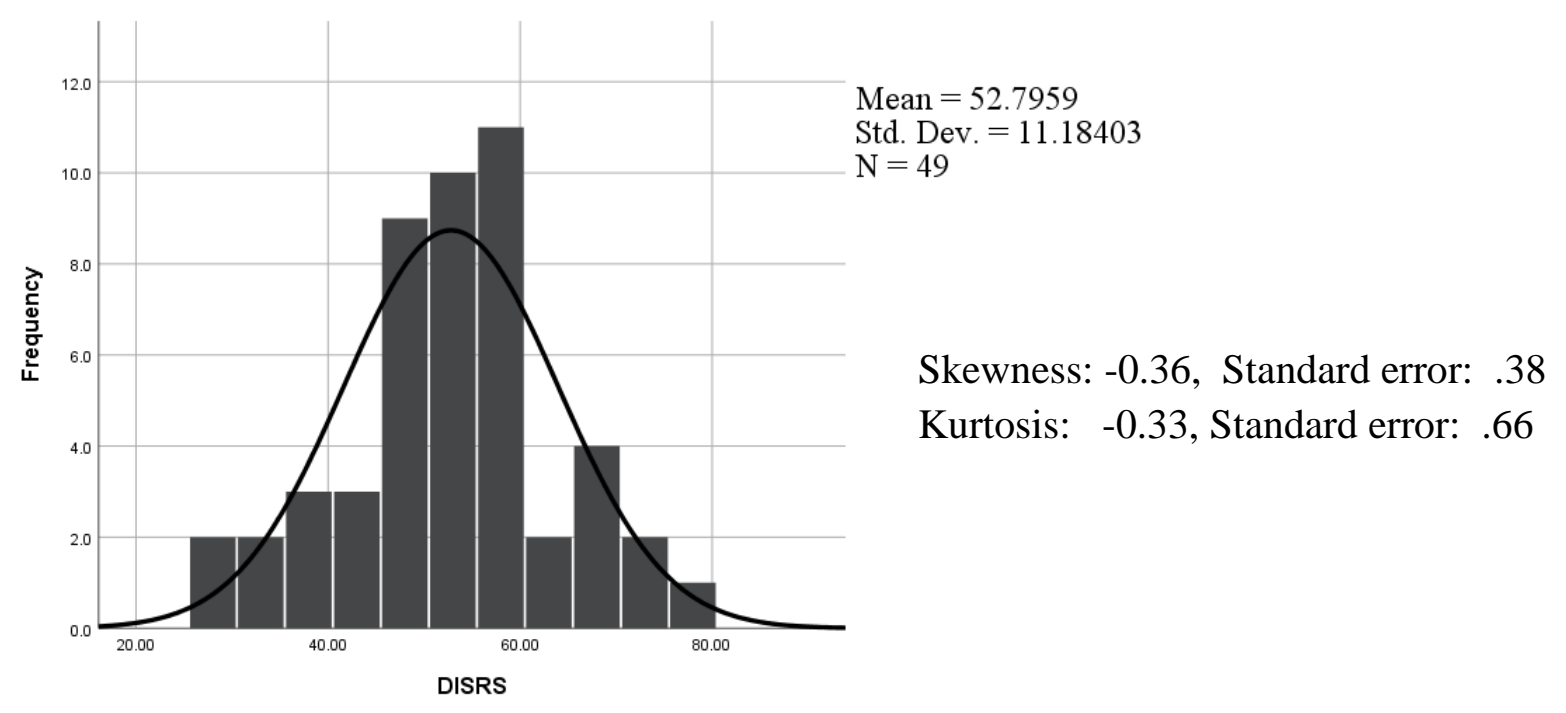

Figure 3.8. Daytime Insomnia Symptoms Response Scale (DISRS) score distribution

The Anxiety and Preoccupation about Sleep Questionnaire (APSQ) was developed to measure the subjective intensity of sleep-specific worry in insomnia (Tang \& Harvey, 2004). The mean and the median scores (73.00 and 76.00) in this sample were higher than the mean scores $(59.39, S D=17.56)$ in a chronic insomnia sample before receiving cognitive behavioural therapy intervention (Harvey, Sharpley, Ree, Stinson, \& Clark, 2007).

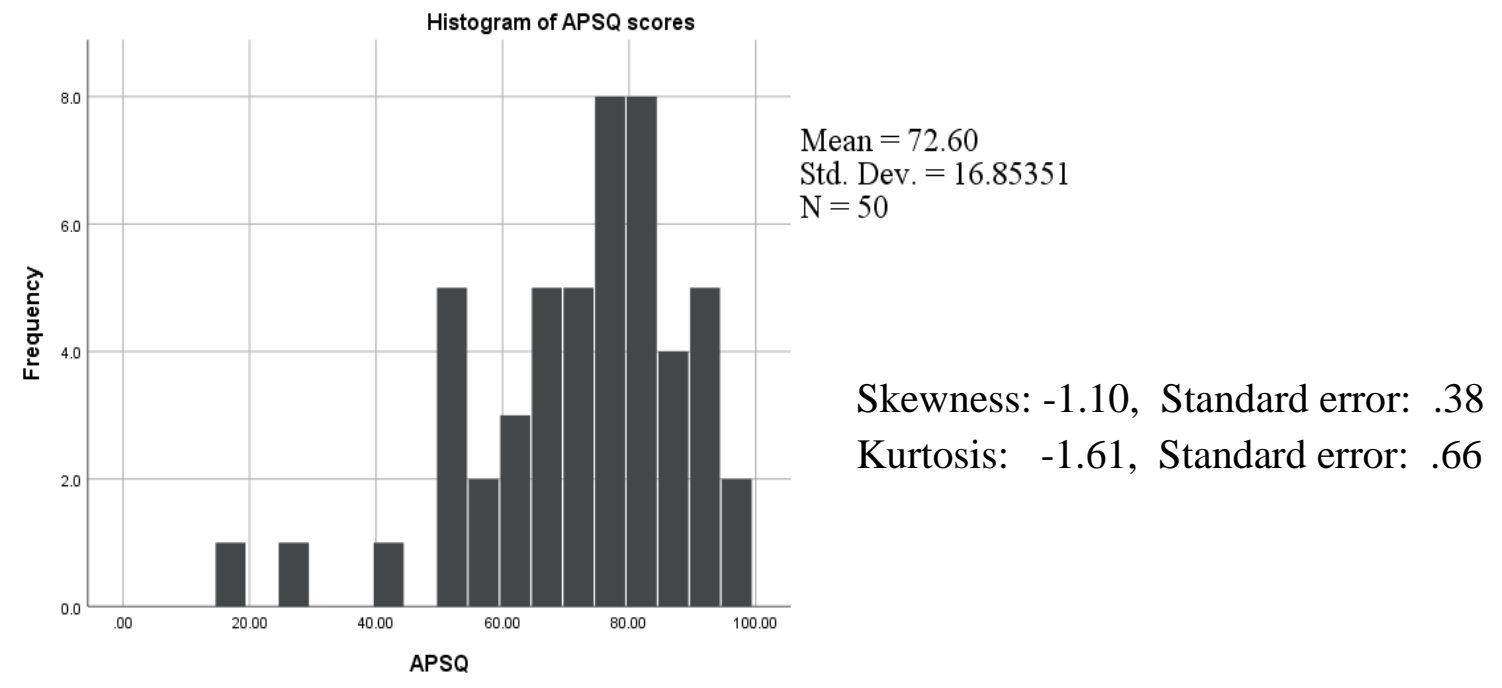

Figure 3.9. Anxiety and Preoccupation about Sleep Questionnaire (APSQ) score distribution 
The Glasgow Sleep Effort Scale (GSES) was developed to assess sleep effort based on the attention-intention-effort model of insomnia (Broomfield \& Espie, 2005). The mean score in this sample was similar to the mean score of the primary insomnia validation sample (7.94 and 7.06 respectively). Each participant in this sample scored higher than the cut-off score of 2 which discriminates between people with insomnia and good sleepers (Blomfield \& Espie, 2005).

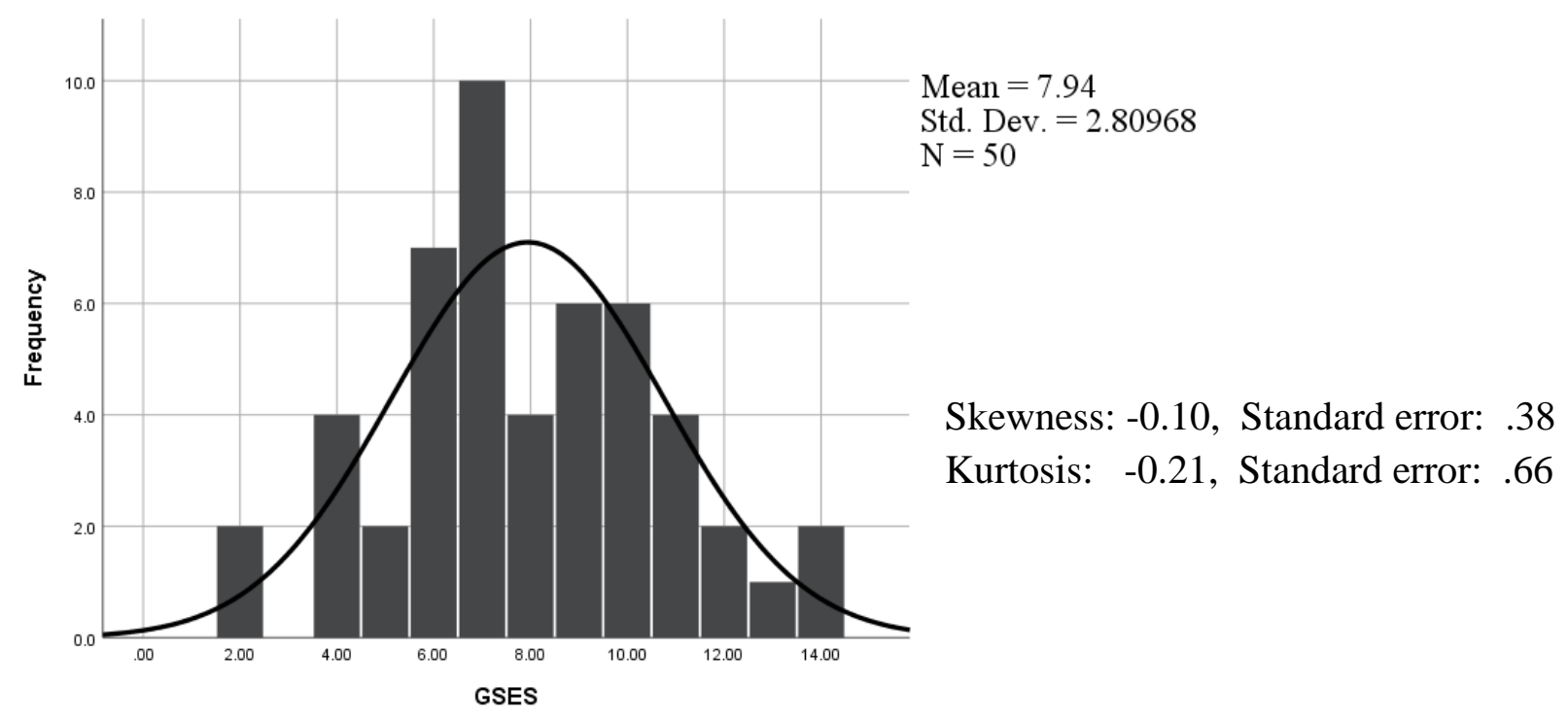

Figure 3.10. Glasgow Sleep Effort Scale (GSES) score distribution

Correlation of Sleep-specific Psychological Variables. As anticipated, there were statistically significant, positive correlations among the sleep-specific psychological variables (DBAS, APSQ, DISRS, and GSES) as well as between these questionnaires and the ISI (Table 3.6). The only exception was the minimal, non-significant relationship between ISI and GSES, $r=.15$ [$.19, .47], p=.29$. 
Table 3.6.

Correlations Among the Insomnia Severity Index and the Cognitive Insomnia Questionnaires

\section{DBAS DISRS APSQ GSES}

\begin{tabular}{|c|c|c|c|c|}
\hline ISI & $.44^{*}$ & $.34 *$ & $.38 * *$ & .15 \\
\hline DBAS & & $.54 * *$ & $.53^{* *}$ & $.31^{*}$ \\
\hline DISRS & & & $.40 * *$ & $.40 *$ \\
\hline APSQ & & & & $.36^{* * *}$ \\
\hline
\end{tabular}

Note. The table presents the Person coefficients for normally distributed variables (ISI, DBAS, DISRS and GSES) and the Kendell's tau for non-normally distributed variables. ISI= Insomnia Severity Index; DBAS = Dysfunctional Beliefs and Attitudes about Sleep Scale; DISRS = Daytime Insomnia Symptoms Response Scale; APSQ = Anxiety and Preoccupation about Sleep Questionnaire; GSES = Glasgow Sleep Effort Scale. ${ }^{*} p<.05 * * p<.01$

\section{Sleep-Specific Psychological Variables in Different Sleep Disorder Diagnostic Categories.}

There were no statistically significant differences in the sleep-specific questionnaire scores across sleep disorder diagnostic categories (DBAS: $\mathrm{F}(2,47)=.20, p=.82$; APSQ: $\mathrm{F}(2,47)=$ $.57, p=.57$; DISRS: $\mathrm{F}(2,46)=.17, p=.87$; GSES: $\mathrm{F}(2,47)=.40, p=.68)$.

\section{Circadian preference.}

The Morningness-Eveningness Scale (MEQ) was developed to assess individual circadian phase preferences (Horne \& Ostberg, 1976). Sixty percent of this sample reported intermediate circadian preference (i.e. neither morning nor evening type); $20 \%$ had scores in the evening chronotype category and the $20 \%$ was in the morning chronotype range. The relationships between the MEQ distribution and age; MEQ scores and DLMO as well as MEQ categories and circadian sleep disorders are presented in the circadian chapter (Chapter 5). 


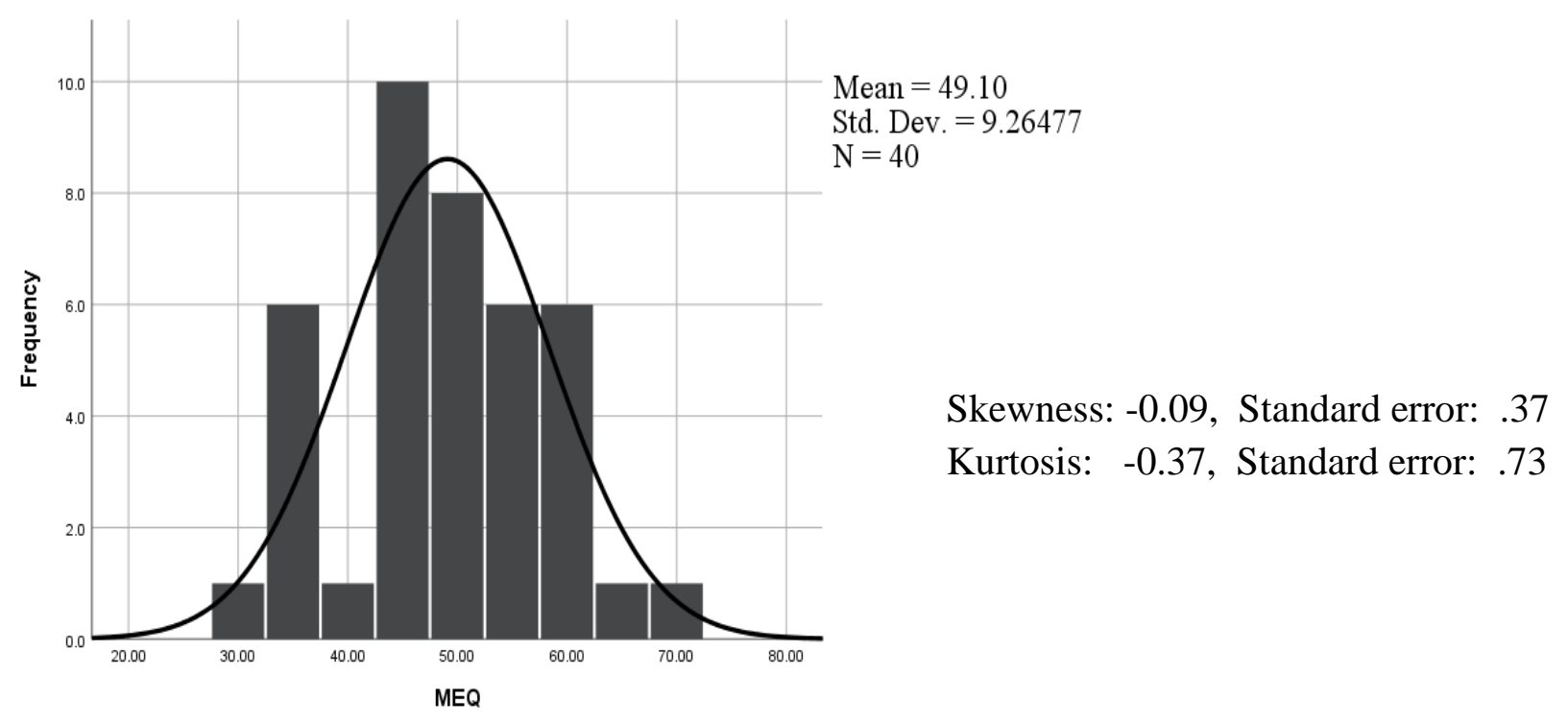

Figure 3.11. Morningness -Eveningness Questionnaire (MEQ) Score Distribution

\section{Visual Analogue Scale (VAS) Ratings}

The VAS scores were normally distributed. The average score distributions are summarized in Table 3.7. In general, participants reported high fatigue and pain before bedtime as well as poor sleep quality and not feeling rested in the morning. Further description and analyses of VAS ratings are provided in Chapter 7. 
Table 3.7.

Average Visual Analogue Scale (VAS) Ratings

Mean $(S D) \quad 95 \%$ BCa CI of the Mean

\begin{tabular}{lcc} 
Fatigue & $61.30(19.49)$ & $56.71-66.42$ \\
Pain & $44.19(19.49)$ & $37.57-50.73$ \\
Sleep Quality & $36.10(11.07)$ & $32.27-40.32$ \\
Refreshed in the Morning & $32.05(11.33)$ & $28.47-35.93$ \\
\hline
\end{tabular}

Note: VAS were $100 \mathrm{~mm}$ lines. The score equals to the distance from the left in millimeters. Higher ratings indicate higher fatigue, more pain, higher sleep quality and more refreshed. $\mathrm{SD}=$ standard deviation; $95 \% \mathrm{BCa} \mathrm{CI}=95 \%$ bias corrected accelerated confidence interval based on 1000 bootstrap samples.

\section{Sleep Diary}

Sleep dairy data is shown in Table 3.8. Eighty eight percent of the sample had a sleep efficiency $^{5}$ (SE) which was lower than $85 \%$. These participants had a sleep onset latency ${ }^{6}$ (SOL) of longer than 30 minutes and/or a wake after sleep onset ${ }^{7}$ (WASO) of at least 30 minutes. The remaining $12 \%$ had normal SOL and sleep continuity according to their sleep diary during the monitoring period, although they reported insomnia symptoms during the clinical interview and their ISI score was in the clinical insomnia range. Most (72\%) of these individuals had sleep abnormalities detected only with PSG and/or with circadian assessment.

\footnotetext{
${ }^{5}$ Sleep efficiency $=($ total sleep time/total time in bed $) \times 100$

${ }^{6}$ Period between sleep attempt and subjective sleep onset

${ }^{7}$ Time spent awake between sleep onset and final awakening
} 
Table 3.8.

Sleep Diary Data

\begin{tabular}{lccc}
\hline & Median (IR) & Mean (SD) & $\begin{array}{c}\text { Bias Corrected } \\
\mathbf{9 5 \%} \text { CI }\end{array}$ \\
\hline SOL (min) & $40.2(28.50)$ & & $38.78-57.92$ \\
WASO (min) & $45.6(49.35)$ & & $36.48-57.60$ \\
\# Awakenings & $2.78(2.29)$ & & $2.44-3.34$ \\
TIB (min) & & $513.27(72.72)$ & $494-531.94$ \\
TST (min) & & 370.12 & $347.76-391.48$ \\
SE (\%) & & $72.43(11.36)$ & $69.26-75.32$ \\
\hline
\end{tabular}

Note: Mean is shown for normally distributed data and median is shown for non- normal distributions. SOL = sleep onset latency (time between bedtime and sleep onset); WASO = wake after sleep onset (time spent awake between sleep onset and final awakening); Awakenings = number of awakenings during the sleep period, not counting the final awakening; TIB = time in bed; TST = total sleep time per night; $\mathrm{SE}=$ sleep efficiency (total sleep time/time in bed); $I R=$ interquartile range; $S D=$ standard deviation; $95 \%$ bias corrected $\mathrm{CI}=95 \%$ bias corrected confidence interval of the measure of central tendency based on 1000 resampling.

Normal values: $\mathrm{SOL} \leq 30 \mathrm{~min}, \mathrm{WASO} \leq 30 \mathrm{~min}, \mathrm{SE} \geq 85 \%$

\section{Actigraphy}

The main actigraphy sleep parameters are summarized in Table 3.9. In brief, the actigraphy median SOL was in the normal range but WASO was higher than normal, resulting in a slightly reduced mean SE commensurate with insomnia.

\section{Correlation between Sleep Diary and Actigraphy Sleep Indices}

Bias corrected and accelerated bootstrap 95\% confidence intervals based on 2000 resampling are reported in square brackets. Sleep diary and actigraphy time in bed (TIB) were 
moderately and significantly related, $\tau=.47[.27, .64], p<.001$. There was also a statistically significant but smaller correlation between sleep diary and actigraphy total sleep time (TST), $\tau=$ $.30[.05, .51], p<.01$. There were weak and non-significant correlations between the sleep diary and actigraphy SOL $(\tau=.19,[-.03, .38] p=.08)$ and sleep diary and actigraphy WASO $(\tau=.19,[-$ $.06, .39] p=.08$ ). Likewise, the relationship between the sleep diary and actigraphy SE was weak and statistically non-significant, $\tau=.17,[-.07, .41], p=.11$.

Table 3.9.

Actigraphy Data

\section{Median (IR) Bias Corrected 95\% \\ CI of median}

\begin{tabular}{lcc}
\hline SOL (min) & $18.07(22.14)$ & $14.80-24.35$ \\
WASO (min) & $39.61(24.17)$ & $35.04-45.75$ \\
\# Awakenings & $32.17(9.62)$ & $30.76-36.70$ \\
TIB (min) & $469.86(58.93)$ & $458.68-481.96$ \\
TST (min) & $421.68(46.46)$ & $427.61-437.50$ \\
SE (\%) & $83.15(5.54)$ & $81.92-84.65$ \\
\hline
\end{tabular}

Note: Median is shown because variables were not normally distributed.

SOL = sleep onset latency (time between bedtime and sleep onset); WASO = wake after sleep onset (time spent awake between sleep onset and final awakening); Awakenings = number of awakenings during the sleep period, not counting the final awakening; TIB = time in bed; TST = total sleep time per night; $\mathrm{SE}=$ sleep efficiency (total sleep time/time in bed); $I R=$ interquartile range; $95 \%$ bias corrected $\mathrm{CI}=95 \%$ bias corrected confidence interval of the median based on 1000 resampling

Normal values: $\mathrm{SOL} \leq 30 \mathrm{~min}, \mathrm{WASO} \leq 30 \mathrm{~min}, \mathrm{SE} \geq 85 \%$ 


\section{Summary of Questionnaire, Sleep Diary and Actigraphy Results}

Insomnia symptoms. The questionnaire results confirmed that participants of this study experienced insomnia symptoms: their highest scores on the RPQ were sleep and fatigue and they all scored above the cut-off score on the ISI recommended for identifying individuals for clinical insomnia studies. Furthermore, the distribution of insomnia types based on the sleep diary data was similar to what has been described in epidemiological research (Pillai, Roth, \& Drake, 2015). Specifically, $88 \%$ of participants in this study had sleep onset insomnia (average $\mathrm{SOL}>30 \mathrm{~min}$ and $\mathrm{SE}<85 \%$ ) or sleep maintenance insomnia (average $\mathrm{SOL}>30 \mathrm{~min}$ and $\mathrm{SE}<$ $85 \%$ ) or combined sleep onset and sleep maintenance insomnia (average SOL > $30 \mathrm{~min}$ and WASO > 30 minutes and SE $<85$ ), while the remaining $12 \%$ had normal sleep pattern according to the diary data but reported sleep onset or sleep maintenance difficulties and poor sleep quality during the intake interview.

The actigraphy results also confirmed the presence of insomnia symptoms but showed less nighttime wakefulness and higher SE than the sleep diary data. In general, patients with insomnia overestimate their wake time on sleep diary compared to PSG; in contrast, actigraphy underestimates wake time compared to the PSG (Baglioni et al., 2013; Lichstein et al., 2006; Marino et al., 2013; Sanchez-Ortuno, Edinger, Means, \& Almirall, 2010a). These general, systematic measurement properties explain the discrepancy between the diary and the actigraphy measures observed in the current study.

With respect to daytime symptoms, fatigue was the leading complaint in the sample. Participants uniformly obtained high scores that were in the clinically significant range on the FSS, showing that every patient (except for only three individuals) struggled with functional limitations which they attributed to their fatigue. The average, prospective VAS fatigue scores 
were also high, showing the participants experienced high fatigue in the evenings during the study period. A less typical finding in an insomnia sample is the high prevalence of subjective excessive daytime sleepiness that was detected in this clinical group - this is further discussed in Chapter 8.

Psychological, sleep-specific variables. From a psychological perspective, participants' scores on the cognitive - behavioural insomnia questionnaires were at least as high as the scores obtained in "primary insomnia" samples. The correlations between these questionnaires and the ISI were also similar to what has been described in primary insomnia, with the only exception of a weak correlation between the GSES and the ISI. These results suggest that psychological factors may have an important role in maintaining insomnia symptoms in this clinical group this is further discussed in Chapter 8.

Psychiatric conditions. In this study, one quarter of the sample had a psychiatric disorder which is the prevalence of psychiatric comorbidity of chronic insomnia in the general population (Ohayon \& Roth, 2003). The percentage of patients diagnosed with major depressive disorder $(14 \%)$ as well as the frequency of self-reported depression symptoms (68\%) was similar to what has been found in other mTBI samples (Osborn, Mathias, \& Fairweather-Schmidt, 2014).

\section{Conclusions}

Overall, this sample has features of both a typical mTBI sample (e.g., causes of TBI and age) and a typical insomnia sample (i.e., gender distribution, prevalence of psychiatric comorbidities, perception of sleep and daytime symptoms as well as psychological factors associated with insomnia). Some of the sample characteristics were atypical of a mTBI sample (e.g., more females than males) or of an insomnia sample (high prevalence of excessive daytime 
sleepiness) - these were the unique features of a clinical group that had both a mTBI and chronic insomnia symptoms. 


\section{Chapter 4: The Use of Polysomnography in the Diagnostic Assessment of Chronic Insomnia following Mild Traumatic Brain Injury}

A decade-old clinical practice guideline recommends that clinicians employ PSG in insomnia evaluation only when there is an indication that the patient has another sleep disorder that may give rise to insomnia symptoms or may be comorbid with insomnia disorder (AASM, 2017; Schutte-Rodin et al., 2008). PSG often shows normal sleep in patients with "primary insomnia" ${ }^{8}$ or if PSG abnormalities are detected, these are less pronounced than what patients report in their sleep diaries (Baglioni et al., 2013). The typical findings on the PSG, for example increased $\mathrm{SOL}^{9}$ and $\mathrm{WASO}^{10}$, increased number of awakenings, or $\mathrm{SE}^{11}$ do not provide information above and beyond what patients report. In other words, the utility of PSG is limited in confirming the presence of insomnia symptoms, a wealth of which can be obtained from patients' subjective reports. This, however, does not discount the importance of PSG in differential diagnosis; i.e., detecting sleep disorders that may fully or partially account for the insomnia symptoms or detecting significant sleep fragmentation about which the patient is oblivious. The challenges with conducting PSG in only a few selected cases of insomnia is that the patient may not report symptoms of other sleep disorders that may cause insomnia symptoms, unless specifically asked or prompted - this often does not occur in non-sleep medical practices. Even when specifically asked, patients may not be aware of symptoms of sleep disorders that lead to insomnia complaints; for example, the specific symptoms of PLMD are often unnoticed by both patients and their bedpartners.

\footnotetext{
${ }^{8}$ Insomnia without a history of psychiatric disorders, sleep disruptive medical comorbidities or other sleep disorders ${ }^{9}$ Sleep Onset Latency: Time between "lights off" and the first sleep epoch

${ }^{10}$ Wake After Sleep Onset: Time spent awake between sleep onset and final awakening

${ }^{11}$ Sleep efficiency $=($ total sleep time/total time in bed $) \times 100$
} 
There is evidence to support the notion that diagnostic interviews and self-report tools are insufficient to detect which patients with insomnia symptoms require PSG assessment. For example, a seminal study concerning the diagnostic utility of PSG in insomnia assessment identified PLMD or obstructive sleep apnea disorder (OSA) in 33\% of treatment-seeking patients with sleep initiation or maintenance insomnia and ruled out a suspected PLMD or OSA in an additional $16 \%$ of the sample; altogether providing diagnostically important information in half of the sample (Edinger, et al., 1989). Most of these patients had been previously treated (and possibly mistreated) for chronic insomnia disorder. Through detailed clinical exam and with use of sleep diaries alone, expert sleep and insomnia specialists could identify only half of those patients with PLMD and one-third of those with OSA (Of note, sleep disordered breathing was likely under-detected in that study because the ambulatory PSG did not measure oxygen desaturation).

In another study, a PSG assessment modified or changed the initial clinical impression of $49 \%$ of patients with chronic insomnia whose symptoms had first been assessed by a sleep expert physicians using a clinical interview, sleep diary and questionnaires (Jacobs, Reynolds, Kupfer, Lovin, \& Ehrenpreis, 1988). In the majority of these cases, the PSG provided new information with regards to nocturnal myoclonus (an old term for PLMD) and in a small percentage, it also revealed undetected OSA. In a more recent study, PSG revealed diagnostic markers of unsuspected sleep disorders in $34 \%$ of patients who had already been assessed by sleep specialists, diagnosed with chronic insomnia disorder, and referred to cognitive behavioural therapy for insomnia as a stand-alone treatment (Cronlein et al., 2012b). PSG findings changed the previously prescribed treatment plan among $21 \%$ of the sample. Three additional studies have reported that OSA was diagnosed in 43-69\% of patients - specifically older adults and 
patients with major depressive disorder - whose self-reported sleep symptoms met DSM or ICSD criteria for chronic insomnia disorder-i.e., they did not report symptoms of OSA (Kinugawa et al., 2012; Lichstein, Riedel, Lester, \& Aguillard, 1999; Ong, Gress, San Pedro-Salcedo, \& Manber, 2009).

In the studies cited above, the initial assessments were conducted by sleep specialists; one can presume that the clinical interviews provided by non-sleep specialist may have a higher percentage of missed diagnoses of OSA and PLMD. Additionally, if in a medical population a base rate of various - non-insomnia - sleep disorders is high, one can speculate that the percentage of missed diagnoses based on interview alone (i.e., diagnosis of insomnia disorder only when in fact, there is an underlying or comorbid other sleep disorder) is increased. Though objective tools are not recommended for routine insomnia diagnosis in general clinical practice, selected objective tools may be required in specific populations, see Figure 1.2.

The only meta-analysis of sleep disorders in TBI came to the conclusion that the prevalence of various sleep disorders appears to be many times higher in TBI samples relative to the base rate in the general population (Mathias \& Alvaro, 2012b). Most of these sleep disorders can cause insomnia symptoms (which would subside if the disorder were treated) or can be comorbid with insomnia disorder (in which case both the insomnia disorder and the comorbid sleep disorder may require specific treatment). There are no studies involving a pure mTBI sample that used insomnia-specific questionnaires, in combination with a clinical interview, sleep diary and PSG to differentiate between insomnia symptoms and insomnia disorder. Consequently, it is unknown what percentage of patients with clinically significant insomnia symptoms also have another (i.e., not insomnia) sleep disorder subsequent to mTBI. This distinction is important with respect to clinical practice. If the prevalence of underlying sleep 
disorders is high, the PSG assessment of these sleep disorders among mTBI patients who present with chronic insomnia symptoms is warranted.

\section{The Objective and Hypothesis of the Current Study}

The first objective of this dissertation was to determine the prevalence of sleep apnea ${ }^{12}$ and PLMD in patients with a history of mTBI and current chronic insomnia symptoms. It was hypothesized that PSG would identify these specific diagnoses (OSA plus PLMD) in the same proportion as in general insomnia samples $(\geq 30 \%)$. A secondary goal was to explore the diagnostic utility and acceptability of home PSG in this patient group.

\section{Method}

Participants. Please see Table 3.1 and a descriptive summary of the sample in Chapter 3.

Procedures (relevant highlights). The study protocol included three nights of PSG recording: one night in the laboratory and two nights in the participants' homes. The laboratory night was recorded 6 or 7 days after the clinical interview at the Sleep and Alertness Clinic in Toronto, see Table 2.1 and Figure 2.1. After the laboratory night, there was an unrecorded night, which was followed by the first home PSG and then the second home PSG.

In the lab, the research staff explained to participants how to conduct the home PSG recording and assisted participants to place the home recording electrode montage. Next, the laboratory technician set up the standard laboratory recording. Participants also kept the Consensus Sleep Diary for the duration of the study, including the PSG nights. At the end of the study, they provided evaluation of the home sleep study experience on Likert type scales and in a descriptive paragraph.

${ }^{12}$ OSA, central and mixed apnea 
Apparatus and measures. The description of the relevant assessment tools and methods, including laboratory and home PSG, STOP-BANG Questionnaire, Epworth Sleepiness Scale, and the Assessment Acceptability Questionnaire can be found in Appendix D.

\section{Analyses}

Descriptive statistics were used to: (1) summarize the frequency of OSA and PLMD diagnoses in the sample; (2) calculate the true positive, true negative, false positive, and false negative rates of the clinical interview and STOP-BANG questionnaire in identifying patients with sleep disorders; (3) cross-tabulate the categorical variables that were thought to be associated with OSA (i.e. age; gender, obesity, snoring; neck size above the cut-off value on the STOP-BANG questionnaire; excessive subjective daytime sleepiness; the diagnosis of high blood pressure); and (4) explore the sleep indices that were subsequently compared in repeated samples analyses.

ANOVA (normally distributed variables) or Friedman's ANOVA (non-normally distributed variables) were employed to compare sleep parameters (i.e., TIB; TST; N1, N2, N3 and REM stages of sleep; number of wake periods; arousal index; REM latency) at the three time points (i.e., sleep lab, first night at home and second night at home). Post-hoc pairwise comparisons with Bonferroni corrections were used to determine if there were significant differences between the laboratory and the home nights. For non-normally distributed variables both the robust parametric $\mathrm{t}$ tests statistics based on 2000 bootstrapping samples and the nonparametric test results are reported, if there was a disagreement between these tests either in significance level or effect sizes. Only the robust test results are reported when there was an agreement between the robust and the non-parametric tests both in terms of significance and effect size. 
Chi-square tests quantified the association between OSA diagnosis and items of the STOP-BANG questionnaire as well as excessive daytime sleepiness. Finally, logistic regression determined the predictive value of the STOP-BANG questionnaire scores on OSA diagnosis.

\section{Results}

Diagnostic findings (i.e., results related to Objective 1). After integrating the PSG findings with the information gathered via the clinical interview and subjective assessment, 22 individuals (44\% of the sample) received the diagnosis of OSA and an additional 4 patients (eight percent) was diagnosed with PLMD. Thus, PSG was required for forming a complete sleep disorder diagnostic opinion in 52\% of the sample. Most of these patients (73\%) had retained a comorbid insomnia disorder diagnosis, while OSA or PLMD appeared to fully account for the insomnia symptoms in $27 \%$ of this group, in which cases comorbid insomnia disorder was not diagnosed. In addition to the diagnostic findings, PSG revealed increased apneahypopnea index (AHI) ${ }^{13}$ in REM sleep and/or in supine position in 4 patients ( $8 \%$ of the sample) and it detected high PLM rate (PLM index >15) in 3 patients ( $6 \%$ of the sample). These individuals were not diagnosed with OSA or PLMD because symptoms did not meet full diagnostic criteria. Nevertheless, these findings became part of the clinical case formulations and may become important for treatment planning.

It is noteworthy, that 28 patients ( 56 percent) of the full sample would have been referred to a PSG study based on the clinical interview and questionnaire data according to the current clinical guidelines. PSG confirmed the diagnosis in 22 of these patients and revealed an additional/different diagnosis in three patients. In addition, PSG detected a sleep disorder in six participants who would not have been referred for a PSG based on the information obtained via

\footnotetext{
${ }^{13}$ Number of apneas and hypopneas per hour
} 
interview and questionnaires. Overall, the clinical interview had 70\% sensitivity and 77\% specificity compared to PSG to detect sleep disorders other than insomnia disorder in this study.

Home versus lab sleep recording (i.e. results related to the minor objective). The home recording procedures, including the users' guide were piloted with the first seven participants. Of the remaining 43 participants, 31 (72\%) provided two nights of home PSG recordings; 10 participants ( $23 \%$ ) conducted one night of scorable recording; and two participants $(5 \%)$ did not record their sleep at home.

There was no significant difference between the time participants spent in bed in the sleep lab, compared to the time they spent in bed at home in the nights when they recorded their sleep with PSG, $F(2,62)=2.08, p=.13, \eta^{2}=.06$. Likewise, there was no significant difference between the amount of time participants were asleep in the lab and the time they spent with sleep during the home recording nights, $F(2,62)=1.34, p=.27, \eta^{2}=.04$. Accordingly, there was no significant difference in SE between the lab and the home nights, $F(2,64)=1.53, p=.22, \eta^{2}=$ .05 or Friedman's $\chi^{2}(2)=1.85, p=.40$. Since TIB and TST were similar at both sleep locations, the absolute values of sleep parameters could be compared (Appendix F).

Though there was no difference in the quantity of sleep between the lab and home nights, participants had lower sleep quality in the lab than they did at home. Specifically, they had more N1 sleep in the lab than in the first night and in the second night at home, while there was no difference between the two nights spent at home, $F(1.58,47.63)=12.88, p<.001, \eta^{2}=.30$. Additionally, they had less N3 sleep in the lab compared to the first or the second night at home, whereas they had a similar amount of deep sleep during the two nights recorded at home, $F(2$, $60)=21.79, p<.01, \eta^{2}=.42$. Furthermore, participants had more wake periods during the night in the lab than at home, whereas there was no difference between the number of wake periods 
during the two home nights, $F(2,64)=10.73, p<.01, \eta^{2}=.25$. As for short arousals, the overall difference in arousal index ${ }^{14}(\mathrm{AI})$ between the lab and home nights approached statistical significance on the omnibus non-parametric test, Friedman's $\chi^{2}(2)=5.63, p=.06$ and was statistically significant using ANOVA, $F(2,64)=5.08, p=.01, \eta^{2}=.14$. Based on both the post-hoc Wilcoxon signed-ranked test and paired sampled t test with 2000 resamples, this difference was caused by the significantly fewer arousals in the second night at home than in the laboratory, $\mathrm{Z}=-3.19, p<.01, r=.51$ or $t(38)=3.3, p<.01, r=.67$, which translates to a large effect. In contrast, there was no significant difference in the arousal index between the laboratory and first night home recordings confirmed by both the non-parametric and the robust, parametric tests, $\mathrm{Z}=0.96, p=.33, r=.15$ and $t(37)=0.99, p=0.33, r=.16$. Although participants had more arousals in the first than in the second night at home, this difference was small and statistically non-significant after Bonferroni corrections were applied, $\mathrm{Z}=1.46, p=0.15, r=.23$ and $t(32)=2, p=0.05, r=.34$.

In addition to having less deep sleep, more wakefulness and more fragmented sleep in the lab, participants also showed difference in their REM sleep between the two sleep locations. Specifically, there was a significant difference in REM latency across the nights, $F(1.71,51.48)$ $=6.25, p<.01, \eta^{2}=.17$ caused by a significantly longer REM latency in the lab than in the first night at home, $t(37)=2.58, p=.01, r=.39$ and the second night at home, $t(37)=3.96, p<$ $0.01, r=.54$. Participants not only entered their first REM cycle later, they also had less REM sleep in the laboratory than at home, $F(1.91,59.40)=5.03, p=.01, \eta^{2}=.14$ both during the first home night, $t(37)=3.32, p<.01, r=.48$; and the second home night, $t(37)=2.37, p=.02, r=$

\footnotetext{
${ }^{14}$ number of arousals per hour
} 
.36. Time spent in stage 2 sleep was similar across the three nights, $F(1.65,49.66)=3.14, p=$ .06.

With respect to the PSG findings with diagnostic value, in most instances (73\%), sleep disordered breathing was detected both in the lab and in the home recordings, while in $11.5 \%$ of the group with disordered breathing, the breathing problem was identified only in the home recordings. The breathing abnormalities captured only at home recordings were exclusively mild OSA based on the AHI for TST, and mildly or moderately increased AHI in REM sleep. Some participants (15.4\% of the OSA group) had only lab recordings. Each of these individuals had at least moderate OSA (AHI > 15), which would have also been captured by the PSG conducted at home. Increased PLM index was mostly (57.1\%) detected only in the laboratory nights, while in some cases (14.3\%) it was detected only with the home PSG recordings or both in the laboratory and at home $(29 \%)$.

On average, participants rated the home PSG recordings as $2=$ "tolerable" on a scale that ranged from $0=$ "extremely burdensome" to $4=$ "highly acceptable". They found the home PSG between "somewhat easy" and "easy" to use (this equals to an average rating of 1.6 on a scale between $0=$ "not easy at all" and $3=$ "very easy"). If they had a choice next time, twothirds of the participants would have preferred home PSG testing, whereas one-third would have chosen to have a PSG recording in the lab. When asked about how the home PSG device would have been easier to use, two themes emerged: (1) getting help in setting up the recording at home and (2) using a device with fewer electrodes. On the positive side, participants found the manual well-written and the procedure well-explained.

Additional results: predictors of OSA. As anticipated, the OSA screening STOPBANG questionnaire scores significantly predicted whether a patient had OSA or did not have 
OSA, $b=1.66$, Wald $\chi^{2}(1)=12.18, p<.01$. The odds ratio shows that with one score increase on the STOP-BANG questionnaire, the change in odds of having OSA versus not having OSA was 5.25; see Table 4.1 below.

Table 4.1.

Coefficients of the model with STOP-BANG score as a predictor whether the individual has obstructive sleep apnea

\begin{tabular}{lcccc}
\hline & $b$ & \multicolumn{2}{c}{$95 \%$ CI for Odds Ratio } \\
& $\begin{array}{l}\text { Lower } \\
\text { i95\% bias corrected confidence } \\
\text { intervals based on 1000 samples] }\end{array}$ & & & Upper \\
\hline Constant & & & & \\
& -3.88 & & & \\
& {$[-6.94,-2.55]$} & 5.25 & 2.07 & 13.33 \\
STOP-BANG & 1.66 & & & \\
score & {$[.84,3.65]$} & & & \\
\hline
\end{tabular}

Obtaining a STOP-BANG score below or above the cut-off recommended for excluding OSA cases in sleep clinic settings (i.e., score 3), significantly predicted if a person had OSA or did not have OSA, $b=2.88$, Wald $\chi^{2}(1)=14.25 p<.01$. Using this cut-off, the logistic regression model could accurately identify $89 \%$ of OSA cases and could correctly classify $68 \%$ of individuals who did not have OSA, yielding an overall correct classification rate of $80 \%$. The sensitivity and specificity of the questionnaire at this cut-off score were $68 \%$ and $89 \%$ compared to the gold standard PSG. A cut-off score of two had higher sensitivity (86\%) and lower specificity $(50 \%)$ in the current sample; these values are similar to those obtained with the cutoff score of three in sleep clinic settings (Nagappa et al., 2015).

When looking at which items of the STOP-BANG questionnaire were uniquely associated with having OSA in this sample, only age (i.e., being 50 or older) showed a significant association with OSA diagnosis, $\chi^{2}(1)=16.64, p<.01$. Based on the odds ratio, the odds of receiving an OSA diagnosis was 32 times higher if patients were at least 50 years of age 
than if they were younger. The cut-off of 50 years on the STOP-BANG questionnaire reflects the increased OSA prevalence in the general population in this age group. However, in the current sample participants who were at least 40 years old were also 13 times more likely to be diagnosed with OSA than patients who were below 40 and even patients who were at least 30 years old were 8.7 times more likely to receive an OSA diagnosis than younger participants. Both 30 years and 40 years of age as cut-off values for categorizing patients into age groups were both significantly associated with OSA diagnosis, $\chi^{2}(1)=8.18, p<.05$ and $\chi^{2}(1)=15.90$, $p<.01$.

With respect to the remaining STOP-BANG items, although a larger percentage of males than females were diagnosed with OSA (55.6\% versus $37.5 \%)$, sex was not significantly associated with having OSA versus not having OSA in this sample, $\chi^{2}(1)=1.52, p=0.22$. Likewise, a higher percentage $(61 \%)$ of those who reported snoring were subsequently diagnosed with OSA compared to those who did not report snoring (34\%); nevertheless, snoring reported by patients was not significantly related to a subsequent OSA diagnosis, $\chi^{2}(1)=3.34, p=.07$. One-tenth of the sample had obesity and 12 percent had hypertension; neither was a unique predictor of OSA diagnosis in the sample. Only one participant reported a history of observed breathing cessations and only two participants had a neck size above the cut-off circumference criterion on the STOP-BANG Questionnaire; therefore, these items did not predict the diagnosis of OSA in this clinical group. Finally, knowing that someone was fatigued was not useful in identifying patients with OSA, since the whole sample, except for three participants, reported significant fatigue-related functional impairment ( $F S S \geq 4$ ). In addition to fatigue, excessive daytime sleepiness is also a symptom of OSA. In the current sample, $45.5 \%$ of patients with OSA reported symptoms of increased sleep propensity on the ESS (ESS $\geq 11)$ and $42 \%$ of 
patients with excessive daytime sleepiness had OSA but subjective excessive daytime sleepiness and OSA were not significantly related, $\chi^{2}(1)=.10, p=.78$.

\section{Discussion}

Sleep disorder diagnoses (Objective 1). This study shows that $52 \%$ of patients with mTBI and chronic insomnia symptoms had a sleep disorder which was dependent on PSG diagnostic assessment. The PSG-dependent diagnosis was mainly OSA (22 individuals or 44\% of the sample), while an additional four patients (eight percent) had PLMD based on ISCD 3 criteria.

The frequency of OSA in this sample is higher than what previous studies described in general insomnia samples (3\% and $22 \%$ ) but is similar to the prevalence of OSA in populations which are known to have particularly high OSA morbidity- specifically older adults with insomnia disorder and patients with major depressive disorder and comorbid insomnia disorder (Kinugawa et al., 2012; Lichstein et al., 1999; Ong et al., 2009). Notably, there were no older adults in the current study and only four participants had comorbid major depressive disorder and OSA; accordingly, old age and depression did not account for the high OSA prevalence in this sample.

With respect to PLMD, the exact population prevalence is unknown. In general, PLMD is relatively rare among individuals below 40 years of age but it is as high as $45 \%$ among older adults (AASM, 2014). In line with this typical age distribution, $75 \%$ of patients diagnosed with PLMD were older than 40 in the current sample. While increased PLM index is commonly associated with certain sleep disorders - for example with restless legs syndrome and OSA- the prevalence of PLMD among individuals with insomnia is less well documented. Studies using 
older (less strict) diagnostic criteria identified $7.7 \%$ - $25 \%$ of patients with chronic insomnia to have PLMD (Cronlein et al., 2012, Edinger et al., 1989, Jacobs et al., 1988).

There is limited data on the prevalence of OSA and PLMD among individuals who sustained mTBI. Only one of the six previous PSG studies involving exclusively individuals with a history of mTBI reported diagnostic PSG data (Rao et al., 2011). That study recruited seven individuals in the acute stage of mTBI who denied having any sleep disorder prior to their TBI. Nevertheless, PSG revealed sleep abnormalities in six of the seven participants: three participants had mild OSA; two participants had increased PLM index; and one had mild OSA and severely elevated PLM index during the recorded night. The PSG results were not integrated with clinical information; therefore, it is not known if these individuals reported symptoms that met diagnostic criteria for OSA disorder or PLMD. One additional study included predominantly individuals who sustained mTBI (Collen, Orr, Lettieri, Carter, \& Holley, 2012). In that study, a consecutive sample of 116 soldiers (85\% with a mTBI and $15 \%$ with moderate or severe TBI) had a sleep assessment in a sleep laboratory. Based on the PSG results, $34.5 \%$ of the soldiers was diagnosed with OSA, while $19.5 \%$ had increased PLM index (the rate of PLMD was not reported). More than half (59\%) of soldiers who were diagnosed with OSA also had insomnia disorder based on DSM-IV criteria. A meta-analysis that pooled sleep data from all TBI severities reported that $25 \%$ of participants had OSA and 2.6 - 35\% had increased PLM rate (not PLMD) defined by standard diagnostic criteria (Mathias \& Alvaro, 2012a). Some of these data were obtained in studies that recruited individuals with poor sleep while in other studies sleep was not assessed before inclusion; none of the studies focused specifically on the comorbidity between insomnia and OSA/PLMD. In comparing the OSA and PLMD percentage in our sample with previous insomnia or TBI data we can conclude that the frequency of OSA is higher 
in the current sample, while the prevalence PLMD may be similar to what has been described in insomnia or in TBI populations.

Possible functional links between OSA and insomnia. The high comorbidity between insomnia and OSA suggests that there may be causal mechanisms between these conditions (unless there is a third variable that explains both): either OSA leads to the insomnia or insomnia contributes to OSA, see Figure 4.1.

OSA could directly give rise to insomnia symptoms of non-restorative sleep, a sense of having "shallow sleep" and frequent awakenings at night (Figure 4.1.; section 1). These subjective symptoms are potentially caused by sleep fragmentation due to respiratory arousals, decreased amount of deep sleep, oxygen desaturation, and PLM arousals. OSA also causes HPA axis hyperactivity and hyperarousal, which in turn, could induce insomnia symptoms in vulnerable individuals (Krakow, Ulibarri, Moore, \& McIver, 2015). Longitudinal studies have shown that positive airway pressure treatment of OSA leads to the resolution of insomnia symptoms in $50 \%$ of patients (Glidewell, Renn, Roby, \& Orr, 2014). The greatest improvement occurs if the patient has sleep maintenance insomnia (i.e. awakenings at night) and severe OSA (Bjornsdottir et al., 2013; Glidewell et al., 2014). This observation confirms that severe OSA in and of itself can cause sleep maintenance insomnia symptoms. When this is the case, it is sufficient to provide effective OSA treatment, which will also eliminate the insomnia symptoms.

An alternative pathway leading from OSA to insomnia may be that OSA triggers insomnia in the first place but insomnia eventually decouples from the OSA and persists as an independent, comorbid entity, see Figure 4.1; section 2. This occurs when the awakenings caused by the respiratory disturbance activate psychological factors (e.g., dysfunctional beliefs about sleep, sleep-focused anxiety; worry and rumination about sleep; sleep effort and non-adaptive 
coping behaviours) that maintain insomnia independently of the OSA. In these situations, insomnia-specific treatment should be provided in addition to OSA treatment.

An opposite direction of relationship between insomnia disorder and OSA may develop if the psycho-physiological arousal and sleep fragmentation in insomnia disorder increases upperairway collapsibility and thus predisposes the individual to have OSA; see Figure 4.1; section 3 (Series, Roy, \& Marc, 1994). 
1. OSA directly causes insomnia symptoms (no insomnia disorder)

OSA

insomnia symptoms

2. OSA triggers acute insomnia which is eventually decouples from OSA (OSA and insomnia disorder)

OSA

$\Rightarrow$ autonomic hyperarousal

$\longrightarrow$ insomnia

dysfunctional beliefs and behaviours

$\longrightarrow$ insomnia disorder

\section{Insomnia disorder leads to OSA}

Insomnia disorder $\longrightarrow$ sleep fragmentation increased upper airway collapsibility OSA

Figure 4.1. Possible Directions of Relationship between OSA and Insomnia 
In considering how the general models may apply to this insomnia clinical group with a history of mTBI, one possibility is that patients experienced insomnia symptoms merely because they had OSA, as shown in Figure 4.1.; section 1. Although this can occur when the OSA is severe and it causes multiple, short awakenings during the night, only $24 \%$ of apnea patients in our sample had sleep maintenance insomnia, while $76 \%$ had sleep onset insomnia only; combined sleep onset and sleep maintenance insomnia; or difficulty with falling back to sleep after their final awakening. Based on the phenomenology of their insomnia, it is unlikely that OSA could fully account for the insomnia symptoms in this group.

It is more likely that in most cases insomnia disorder developed as a comorbid condition (Figure 4.1.; section 2) and variables related to the brain injury may have mediated the relationship between OSA and insomnia disorder. On the physiological level, pain - which is a common symptom following the injury - may cause sleep fragmentation, compounding the effects of OSA (Bjurstrom \& Irwin, 2016). Therefore, patients with OSA who have post-injury pain may be at an increased risk for developing insomnia compared to those without OSA. Indeed, in this sample $85 \%$ of patients with chronic pain had both OSA and insomnia disorder. It is also important to know that sedative analgesics and depressants prescribed after the injury to treat pain and improve mood can worsen untreated sleep apnea and compromise sleep quality (Bohra, Kaushik, Temple, Chung, \& Shapiro, 2014).

On a psychological level, the distress caused by the injury and its impact on health and everyday functioning can increase psycho-physiological arousal and intensify the effects of OSA and pain. Once the insomnia appears, it may also be maintained by sleep-specific psychological factors, for example sleep-focused anxiety and worry about sleeplessness. Indeed, participants with OSA endorsed dysfunctional beliefs and attitudes about sleep; anxiety and preoccupation 
about sleep; rumination abut insomnia symptoms and sleep effort to the same extent as participants who did not have OSA, which suggest psychopathological factors may have been contributing to their insomnia. If patients are on sick leave after the injury, they also have more freedom to engage in non-adaptive coping behaviours, for example spending excessive time in bed, having long naps, or decreased social and physical activity. These non-adaptive coping behaviours can worsen insomnia and pain and contribute to the functional impairment as shown in Figure 4.2.

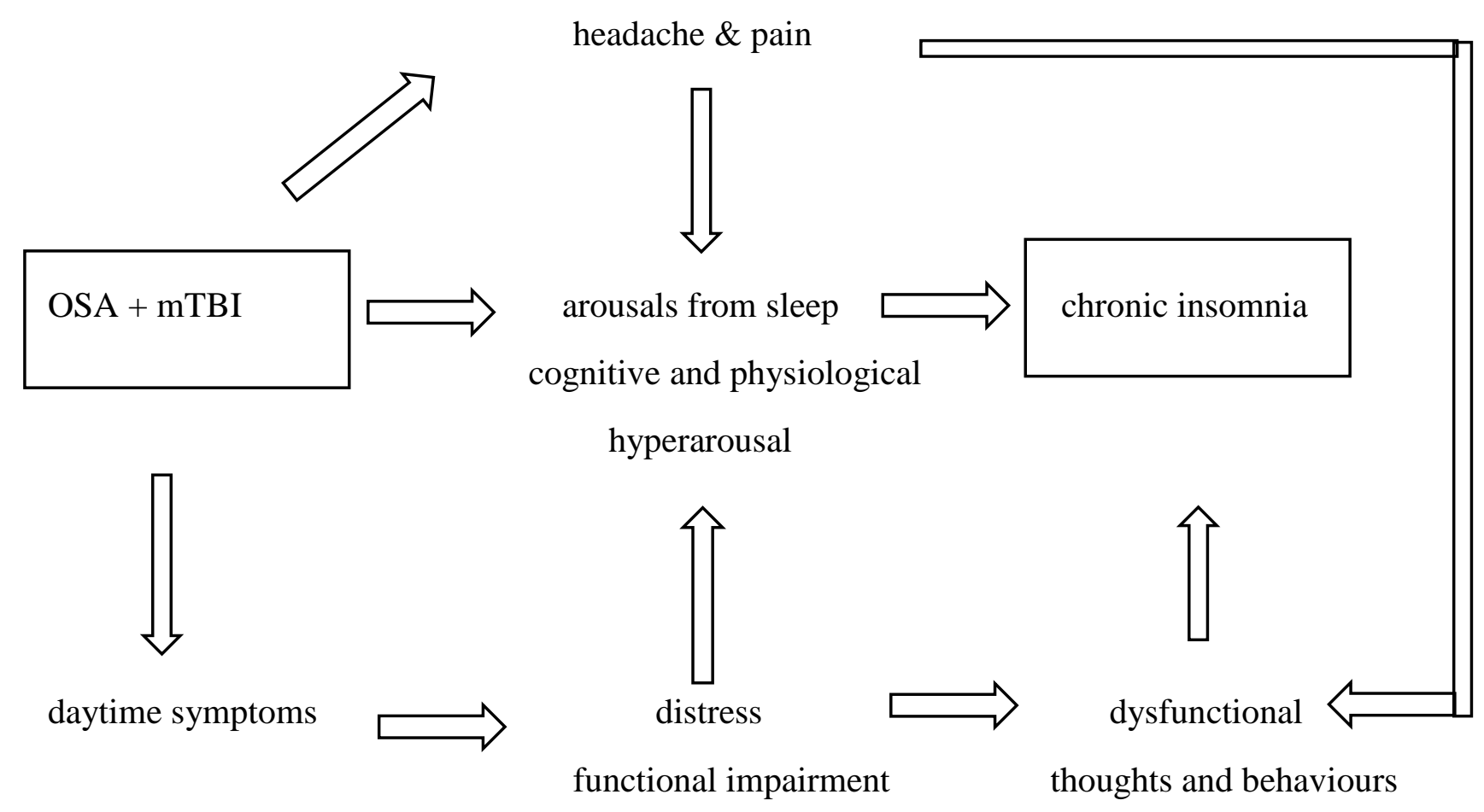

Figure 4.2. Theoretical pathways between OSA and Insomnia following mTBI

An adjunct explanation for the high frequency of co-occurring chronic insomnia and OSA in this sample is that patients who have both disorders may be more likely receive a referral for a sleep assessment than those who have OSA or insomnia symptoms only. People who have OSA without insomnia may not receive a referral to a sleep clinic because the sleepiness, fatigue, 
and cognitive problems caused by the OSA (and amplified by the mTBI) will likely be attributed to the brain injury in clinical settings where TBI care is provided. Likewise, insomnia is often not seen as a significant medical issue on its own right that would merit referral to a sleep clinic.

However, when both conditions are present, the distress caused by the insomnia symptoms and the noticeable functional limitations due to daytime sleepiness, extreme fatigue, significant cognitive difficulties and mood problems caused by both sleep disorders- are alarming for both the individual and the concussion care provider and point to a possible sleep issue that requires sleep assessment, as illustrated in Figure 4.3 below.
A. Undetected OSA before the head injury
B. The OSA symptoms manifest

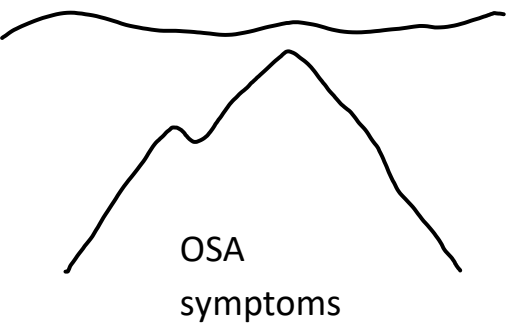

following the head injury

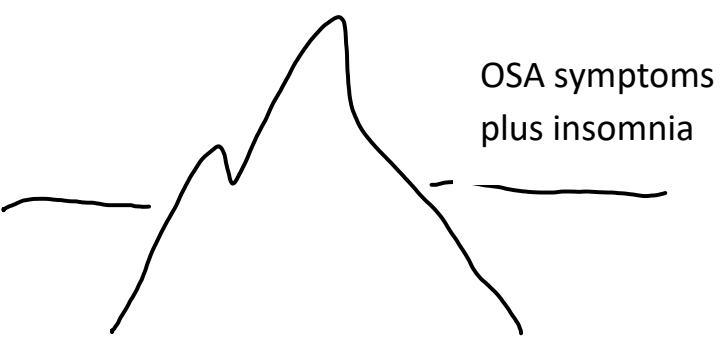

Figure 4.3. Undiagnosed OSA manifests following a mTBI

This figure shows that OSA symptoms may be undetected before TBI, as an iceberg below the surface of the water. The brain may not be able to compensate for the combined burden of OSA and TBI and symptoms become apparent after the injury, as the iceberg becomes visible. If the patient also has insomnia symptoms, the sleep difficulty may draw attention to the possible sleep origin of the symptoms and may trigger a referral for a sleep assessment.

Discussion of home sleep testing results (minor objective). PSG is the gold-standard tool in the diagnostic assessment of OSA and PLMD. PSG is traditionally conducted in sleep laboratories and evaluated according to standard criteria. While laboratory PSG is the standard method to assess OSA and PLMD, it has some drawbacks, including: (1) access to sleep 
laboratories is limited in many geographical regions; (2) wait times can be long; (3) the assessment is costly because of the human resources required for conducting the sleep study and evaluating the results.

Home sleep testing has recently gained popularity as an affordable alternative for laboratory-based PSG. Portable monitors that record airflow, respiratory effort and blood oxygenation can be used for the diagnostic assessment of OSA as part of a comprehensive clinical evaluation (Schutte-Rodin et al., 2008). Unattended home PSG with automatic scoring has the advantages of both a PSG (EEG and leg movement recording) and the home monitors (affordable and operated at home by the patient). Home PSG has particularly high validity in the sleep assessment of individuals with insomnia disorder because sleep in insomnia has increased night-to-night variability, which is better captured with home than with lab PSG (Edinger et al., 1997; Hirscher et al., 2015).

Most participants (95\%) in this study provided at least one scorable home PSG recording and three quarters recorded two nights without any technical assistance at home. This is the same success rate as was previously reported in the literature in studies where the set-up was provided in the laboratory or at home by sleep technicians (Bruyneel, Libert, Ameye, \& Ninane, 2015). The fact that the participants in the current study were able to set up the recording without professional assistance at home suggests that subjective post-concussion cognitive difficulties do not preclude the use of unassisted home PSG as part of a sleep assessment following mTBI.

With regards to their sleep architecture, participants of the current study had more "light" N1 sleep; less deep N3 sleep and more awakenings during the night in the laboratory compared to the nights recorded at home. In contrast, the comparison between home PSG recordings did not show systematic difference between the two nights with regards to sleep continuity; sleep 
fragmentation; PSG and sleep diary sleep efficiency; or sleep architecture. Previous research has shown that there is no "first night effect" when PSG recording is conducted at home, which implies that it is the unusual sleep environment in the laboratory rather than sleeping with an equipment that causes low sleep quality during the first night in the lab (Edinger, et al., 1997). In line with these findings, the results of the current study suggest that there is no "first night effect" when home recordings are conducted in post-concussion chronic insomnia; however, one has to keep in mind that in this study that the lab and home nights were not counter-balanced.

In terms of its diagnostic utility, home PSG provided results that were consistent with the sleep laboratory findings with regards to OSA detection, which replicates previous findings in patients with sleep apnea (Banhiran, Chotinaiwattarakul, Chongkolwatana, \& Metheetrairut, 2014) . Furthermore, it detected mild sleep apnea in total sleep time or high AHI in REM sleep when the laboratory sleep recordings were normal. Previous research has shown that AHI was higher in the home than in the laboratory, which could explain why mild sleep-related breathing issues are better captured with home PSG than with lab PSG (Banhiran et al., 2014). However, there was no significant difference between the lab and the home nights in terms of total AHI in the current sample. A more likely explanation is that patients may have been more relaxed when they slept at home - indicated by less light sleep, more deep sleep, less awakenings, and lower arousal rate in the home recordings. A deeper muscle relaxation may have allowed the mild OSA to manifest during the home recordings.

In contrast to the consistency between the laboratory and home PSG in detecting OSA, only 29\% of high PLM index was captured in both settings, whereas 57\% was captured only in the sleep laboratory and $14 \%$ only at home. This may have occurred by chance, since the there is a high nigh-to-night variability of PLM (Trotti et al., 2009). Since PLM typically occurs in N1 
and N2 sleep, it is also possible that the PLM index was higher in the lab because participants spent longer time in N1 sleep in the laboratory than they did at home.

It is noteworthy that the sleep disorder diagnostic results were consistent between the two home nights, which suggests that one night of home recording provides a good representation of sleep and multiple home recordings are not required for clinical diagnostic purposes.

Sleep apnea screening may gain an important role in identifying patients with chronic insomnia who likely have OSA and need PSG assessment following a mTBI. The OSA screening STOP-BANG Questionnaire score $\geq 3$ had $89 \%$ specificity with regards to OSA diagnosis in this sample compared to PSG. Based on the positive likelihood ratios, there was moderate increase of likelihood that someone with a score $\geq 3$ had OSA. Accordingly, the study results suggest that STOP-BANG is a useful tool to identify individuals who likely have OSA in this clinical group. Specifically, if patients with post-concussion insomnia obtain a score $\geq 3$ on the STOP-BANG Questionnaire, they likely have OSA and should be referred to further sleep assessment. Based on its sensitivity and negative likelihood ratio, the cut-off score of 2 could be used to rule out OSA. These cut-off scores are lower than the one recommended for use in sleep clinics - see Appendix D (Nagappa et al., 2015). Given the relatively small sample size of this study, further research will need to examine the psychometric properties of the STOP-BANG Questionnaire among patients who seek assessment for chronic insomnia following a mTBI.

\section{Chapter Summary and Conclusions}

This study showed that half of the patients reporting symptoms of chronic insomnia after a mTBI had a specific sleep disorder (OSA or PLMD) that required PSG for diagnostic assessment. This finding shows that PSG has a crucial role in the sleep evaluation of patients who report chronic insomnia symptoms following a mTBI. Home PSG appears to be a feasible 
alternative to laboratory PSG with some possible advantages (e.g., patient preference, superior OSA diagnosis, and high ecological validity) and drawbacks (less likely to detect PLMD).

Future research should further examine the psychometric properties of OSA screening tools and compare home and laboratory PSG in patients who experience persistent insomnia symptoms following a mTBI. 


\section{Chapter 5: Circadian Sleep-Wake Phase Disorders among Individuals with Chronic Insomnia Following Mild Traumatic Brain Injury}

\section{Circadian Sleep-Wake Disorders among Patients with a History of TBI}

In the field of TBI, human studies concerning melatonin secretion have predominantly been conducted in the context of severe TBI, see Table 5.1 (Ayalon et al., 2007; Grima, Ponsford, St Hilaire, Mansfield, \& Rajaratnam, 2016; Guaraldi et al., 2014; Paparrigopoulos et al., 2006; Paul \& Lemmer, 2007; Seifman et al., 2008; Shekleton et al., 2010; Steele, Rajaratnam, Redman, \& Ponsford, 2005)

These studies showed that the daily melatonin rhythm was absent or attenuated among patients who are treated in an intensive care unit (ICU) following a severe TBI (Paparrigopoulos et al., 2006; Paul \& Lemmer, 2007). Melatonin concentrations measured in these studies were lower compared to melatonin values in non-TBI ICU controls and healthy controls. Another consistent finding of this line of research is that melatonin secretion was most impaired (based on its concentration and lack of daily rhythm) in patients who had the worst clinical status (Guaraldi et al., 2014; Paparrigopoulos et al., 2006; Seifman et al., 2008). It is likely to be a combination of the neurological impact of the injury and environmental factors (e.g., diminished 24-hour environmental light-dark cycle and administration of medications that influence melatonin secretion) that contribute to impaired melatonin production of patients in the ICU. 
Table 5.1.

Summary of Studies Assessing Melatonin Secretion and Circadian Sleep-wake Disorders Following Traumatic Brain Injury

\begin{tabular}{|c|c|c|c|c|c|}
\hline Authors (year) & Study sample & $\begin{array}{c}\text { Sample } \\
\text { size }\end{array}$ & $\begin{array}{c}\text { Method of melatonin } \\
\text { assessment }\end{array}$ & Main findings & Limitations \\
\hline $\begin{array}{l}\text { Paparrigopoulus et } \\
\text { al. (2006) }\end{array}$ & $\begin{array}{l}\text { Sedated and } \\
\text { mechanically } \\
\text { ventilated patients } \\
\text { at the ICU } \\
\text { following severe } \\
\text { TBI }\end{array}$ & 8 & $\begin{array}{l}\text { Blood samples were } \\
\text { collected in every four } \\
\text { hours from } 8 \text { am on } \\
\text { the first day of ICU } \\
\text { admission for two } \\
\text { consecutive days } \\
\text { 24-hour serum } \\
\text { melatonin profile was } \\
\text { determined }\end{array}$ & $\begin{array}{l}\text { Reduced peak and mean } \\
\text { melatonin concentrations } \\
\text { compared to normal values. } \\
\text { Four patients with GCS } \\
\text { score < } 7 \text { had flat melatonin } \\
\text { profiles, whereas the four } \\
\text { participants with GCS > } 7 \\
\text { retained the normal diurnal } \\
\text { fluctuation of melatonin } \\
\text { concentration. } \\
\text { GCS and APACHE II } \\
\text { scores had significant } \\
\text { correlations with mean } \\
\text { melatonin concentrations } \\
\text { and mean day/night } \\
\text { melatonin ratios }\end{array}$ & $\begin{array}{l}\text { Small sample size } \\
\text { and large individual } \\
\text { normal variations in } \\
\text { melatonin secretion } \\
\text { may have } \\
\text { confounded the } \\
\text { comparison with } \\
\text { literature values } \\
\text { Patients were on } \\
\text { medications that } \\
\text { may have affected } \\
\text { their melatonin } \\
\text { secretion } \\
\text { Area under the } \\
\text { melatonin curve } \\
\text { was not determined }\end{array}$ \\
\hline & & & & $\begin{array}{l}\text { There was no correlation } \\
\text { between melatonin and core } \\
\text { body temperature }\end{array}$ & \\
\hline
\end{tabular}

Note: ICU = intensive care unit; TBI = traumatic brain injury; GSC = Glasgow Coma Scale; APACE II = Acute Physiology and Chronic Health Evaluation 
Table 5.1 (cont.)

Summary of studies assessing melatonin secretion and circadian sleep-wake disorders following traumatic brain injury

\begin{tabular}{|c|c|c|c|c|c|}
\hline Authors (year) & Study sample & $\begin{array}{l}\text { Sample } \\
\text { size }\end{array}$ & $\begin{array}{c}\text { Method of melatonin } \\
\text { assessment }\end{array}$ & Main findings & Limitations \\
\hline $\begin{array}{l}\text { Paul \& Lemmer } \\
\text { (2007) }\end{array}$ & $\begin{array}{l}\text { Sedated and } \\
\text { ventilated patients } \\
\text { following severe } \\
\text { TBI or cerebral } \\
\text { hemorrhage and a } \\
\text { control group of } \\
\text { participants } \\
\text { following } \\
\text { surgery/non-brain } \\
\text { trauma/acute } \\
\text { respiratory distress } \\
\text { in the same } \\
\text { environment at the } \\
24 \text { bed ICU unit }\end{array}$ & 24 & 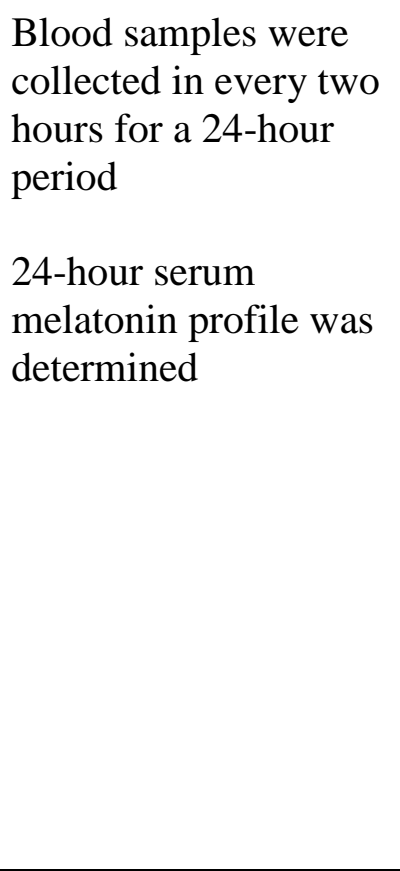 & $\begin{array}{l}\text { Lower mean melatonin } \\
\text { concentrations in the brain } \\
\text { injury group compared to } \\
\text { the ICU control group } \\
\text { Disturbed or missing } \\
\text { melatonin rhythm, } \\
\text { especially in the brain injury } \\
\text { group } \\
\text { Lack of normal circadian } \\
\text { rhythm of blood pressure, } \\
\text { heart rate, serum cortisol, } \\
\text { and core body temperature. } \\
24 \text { hour mean blood } \\
\text { pressure was higher and } \\
\text { heart rate was lower in the } \\
\text { brain injury group. }\end{array}$ & $\begin{array}{l}\text { Patients were on } \\
\text { medications that } \\
\text { may have affected } \\
\text { their melatonin } \\
\text { secretion and } \\
\text { cardiovascular } \\
\text { functions. } \\
\text { Area under the } \\
\text { melatonin curve } \\
\text { was not determined }\end{array}$ \\
\hline
\end{tabular}

Note: $\mathrm{ICU}=$ intensive care $\mathrm{unit} ; \mathrm{TBI}=$ traumatic brain injury 
Table 5.1 (cont.)

Summary of studies assessing melatonin secretion and circadian sleep-wake disorders following traumatic brain injury

\begin{tabular}{|c|c|c|c|c|c|}
\hline Authors (year) & Study sample & $\begin{array}{c}\text { Sample } \\
\text { size }\end{array}$ & $\begin{array}{c}\text { Method of melatonin } \\
\text { assessment }\end{array}$ & Main findings & Limitations \\
\hline $\begin{array}{l}\text { Seifman et al. } \\
(2008)\end{array}$ & $\begin{array}{l}\text { Sedated patients } \\
\text { following severe } \\
\text { TBI at the ICU and } \\
\text { controls (patients } \\
\text { before elective } \\
\text { brain surgeries, for } \\
\text { example clipping of } \\
\text { unruptured MCA } \\
\text { aneurism, insertion } \\
\text { of VP shunt or } \\
\text { decompression of } \\
\text { trigeminal } \\
\text { neuralgia) }\end{array}$ & $\begin{array}{c}59 \\
\text { (39 TBI } \\
\text { and } 20 \\
\text { controls) }\end{array}$ & $\begin{array}{l}\text { Blood samples were } \\
\text { taken in the first } 10 \\
\text { post-injury days at } 9 \\
\text { am (TBI patients) } \\
\text { One CSF and blood } \\
\text { sample was collected } \\
\text { from the control group } \\
\text { Mean group CSF and } \\
\text { serum melatonin } \\
\text { concentrations were } \\
\text { calculated }\end{array}$ & $\begin{array}{l}\text { CSF mean melatonin } \\
\text { concentration showed a } \\
\text { biphasic curve, with peaks } \\
\text { at day } 2 \text { and } 8 \text { post-injury } \\
\text { Patients with more severe } \\
\text { injury had lower melatonin } \\
\text { levels }\end{array}$ & $\begin{array}{l}\text { Patients were on } \\
\text { medications that } \\
\text { may have affected } \\
\text { their melatonin } \\
\text { secretion and } \\
\text { cardiovascular } \\
\text { functions. } \\
\text { The control group } \\
\text { was older than the } \\
\text { TBI group and the } \\
\text { control group had } \\
\text { lower than normal } \\
\text { melatonin. } \\
\text { Only one sample } \\
\text { per day was } \\
\text { analyzed; therefore } \\
\text { diurnal changes in } \\
\text { melatonin levels } \\
\text { could not be } \\
\text { captured. }\end{array}$ \\
\hline
\end{tabular}

Note: $\mathrm{ICU}=$ intensive care unit; TBI = traumatic brain injury; $\mathrm{CSF}$ = cerebrospinal fluid 
Table 5.1. (cont.)

Summary of studies assessing melatonin secretion and circadian sleep-wake disorders following traumatic brain injury

\begin{tabular}{|c|c|c|c|c|c|}
\hline Authors (year) & Study sample & $\begin{array}{c}\text { Sample } \\
\text { size }\end{array}$ & $\begin{array}{c}\text { Method of melatonin } \\
\text { assessment }\end{array}$ & Main findings & Limitations \\
\hline $\begin{array}{l}\text { Guaraldi et al. } \\
\text { (2014) }\end{array}$ & $\begin{array}{l}\text { Patients in } \\
\text { vegetative state 6- } \\
18 \text { months } \\
\text { following severe } \\
\text { TBI and healthy } \\
\text { controls } \\
\text { Lesions were in the } \\
\text { cortex and affected } \\
\text { the thalamo-cortical } \\
\text { connection, while } \\
\text { the hypothalami } \\
\text { were preserved }\end{array}$ & $\begin{array}{c}15 \\
\text { (six } \\
\text { patients } \\
\text { and nine } \\
\text { controls) }\end{array}$ & $\begin{array}{l}\text { Serum melatonin } \\
\text { concentration was } \\
\text { determined from } \\
\text { blood samples taken } \\
\text { hourly between } 0.30 \\
\text { am and } 3.30 \text { am at the } \\
\text { baseline (light } \\
\text { intensity < } 5 \text { lux) and } \\
\text { on the experimental } \\
\text { night ( } 470 \text { nm blue } \\
\text { light exposure). }\end{array}$ & $\begin{array}{l}\text { Serum melatonin } \\
\text { significantly increased } \\
\text { between } 0.30 \text { am and } 3.30 \\
\text { am in the control but not in } \\
\text { the TBI group. } \\
\text { Serum melatonin was lower } \\
\text { (significantly by } 3.30 \text { am) } \\
\text { in the TBI group. } \\
\text { Significant suppression of } \\
\text { melatonin by blue light by } 3 \\
\text { am in the healthy but not in } \\
\text { the TBI group. } \\
\text { There was a significant } \\
\text { positive correlation between } \\
\text { CRS-R and plasma } \\
\text { melatonin at } 3.30 \text { am, } \\
\text { showing the patients in } \\
\text { better clinical state had } \\
\text { higher melatonin } \\
\text { concentration. }\end{array}$ & $\begin{array}{l}\text { Patients were on } \\
\text { medications that } \\
\text { may have affected } \\
\text { their melatonin } \\
\text { secretion. } \\
\text { Only four samples } \\
\text { were taken and } \\
\text { therefore the } \\
\text { melatonin secretion } \\
\text { curve and light } \\
\text { response curve } \\
\text { could not be } \\
\text { determined. }\end{array}$ \\
\hline
\end{tabular}

Note: $\mathrm{TBI}=$ traumatic brain injury; CRS-R = Coma Recovery Scale - Revised 


\begin{tabular}{|c|c|c|c|c|c|}
\hline Authors (year) & Study sample & $\begin{array}{c}\text { Sample } \\
\text { size }\end{array}$ & $\begin{array}{c}\text { Method of melatonin } \\
\text { assessment }\end{array}$ & Main findings & Limitations \\
\hline Steele et al. (2005) & $\begin{array}{l}\text { Individuals on } \\
\text { average } 17 \text { months } \\
\text { post-TBI recruited } \\
\text { during their } \\
\text { outpatient } \\
\text { rehabilitation } \\
\text { appointments. } \\
\text { Injury severity } \\
\text { ranged from mild to } \\
\text { severe. Participants } \\
\text { were not selected } \\
\text { based on sleep } \\
\text { history. }\end{array}$ & $\begin{array}{c}20 \\
(10 \\
\text { patients } \\
\text { and } 10 \\
\text { healthy } \\
\text { controls })\end{array}$ & $\begin{array}{l}\text { DLMO test: salivary } \\
\text { melatonin } \\
\text { concentration was } \\
\text { determined from } \\
\text { samples taken in } \\
\text { every } 30 \text { minutes } \\
\text { between } 18.00 \text { and } \\
00.30 \text { under dim light } \\
\text { conditions. }\end{array}$ & $\begin{array}{l}\text { There was no } \\
\text { difference between } \\
\text { mean subjectively } \\
\text { reported sleep } \\
\text { schedule and DLMO } \\
\text { in the two groups. } \\
\text { In four of the } 10 \text { TBI } \\
\text { patients DLMO could } \\
\text { not be determined } \\
\text { because melatonin did } \\
\text { not rise. } \\
\text { There was significant } \\
\text { correlation between } \\
\text { DLMO and } \\
\text { Morningness- } \\
\text { Eveningness } \\
\text { Questionnaire Scores. }\end{array}$ & $\begin{array}{l}\text { Number of participants } \\
\text { with } \\
\text { mild/moderate/severe TBI } \\
\text { was not reported. } \\
\text { Only group means were } \\
\text { compared. Based on the } \\
\text { range of DLMOs and the } \\
\text { range of habitual sleep } \\
\text { and wake times, there } \\
\text { may have been } \\
\text { individuals with } \\
\text { somewhat advanced or } \\
\text { delayed rhythm in both } \\
\text { groups. Given the small } \\
\text { TBI sample size, showing } \\
\text { individual curves and } \\
\text { associated average sleep } \\
\text { onset and wake times } \\
\text { would have been } \\
\text { informative. }\end{array}$ \\
\hline
\end{tabular}

Note: $\mathrm{TBI}=$ traumatic brain injury; DLMO = dim light melatonin onset 


\begin{tabular}{|c|c|c|c|c|c|}
\hline Authors (year) & Study sample & $\begin{array}{l}\text { Sample } \\
\text { size }\end{array}$ & $\begin{array}{c}\text { Method of melatonin } \\
\text { assessment }\end{array}$ & Main findings & Limitations \\
\hline $\begin{array}{l}\text { Shekleton et al. } \\
\text { (2010) }\end{array}$ & $\begin{array}{l}\text { Individuals six } \\
\text { months following } \\
\text { TBI (most had } \\
\text { severe TBI, some } \\
\text { had moderate) } \\
\text { attending } \\
\text { rehabilitation. } \\
\text { Patients were not } \\
\text { selected based on } \\
\text { sleep history. } \\
\text { Patients with high } \\
\text { risk of sleep apnea } \\
\text { (based on } \\
\text { questionnaire } \\
\text { scores) were } \\
\text { excluded. }\end{array}$ & $\begin{array}{c}18 \\
\text { (nine } \\
\text { patients } \\
\text { and nine } \\
\text { healthy } \\
\text { controls) }\end{array}$ & $\begin{array}{l}\text { DLMO test: salivary } \\
\text { melatonin } \\
\text { concentration was } \\
\text { determined from } \\
\text { samples taken in } \\
\text { every } 30 \text { minutes } \\
\text { between } 18.00 \text { and } \\
00.30 \text { under dim light } \\
\text { conditions. }\end{array}$ & $\begin{array}{l}\text { There was no difference } \\
\text { between the DLMO in the } \\
\text { two groups. } \\
\text { The TBI group produced } \\
\text { less melatonin (based on } \\
\text { the area under the partial } \\
\text { curve) than the control } \\
\text { group. } \\
\text { DLMO could not be } \\
\text { determined in nine } \\
\text { participants in each group } \\
\text { (may have been outside of } \\
\text { the normal range). } \\
\text { The TBI group had lower } \\
\text { subjective sleep quality } \\
\text { than controls. } \\
\text { Patients with TBI had } \\
\text { higher SWS than controls. } \\
\text { PSG WASO and SE were } \\
\text { not significantly different } \\
\text { between the groups after } \\
\text { controlling for depression } \\
\text { questionnaire scores. }\end{array}$ & $\begin{array}{l}\text { Sleep diary and } \\
\text { actigraphy data was } \\
\text { not collected to } \\
\text { assess sleep-wake } \\
\text { schedule. } \\
\text { Sleep disorders were } \\
\text { not assessed/reported. }\end{array}$ \\
\hline
\end{tabular}

Note: $\mathrm{TBI}$ = traumatic brain injury; DLMO = dim light melatonin onset; PSG = polysomnography; WASO = wake after sleep onset; $\mathrm{SE}=$ sleep efficiency 


\begin{tabular}{|c|c|c|c|c|c|}
\hline Authors (year) & Study sample & $\begin{array}{c}\text { Sample } \\
\text { size }\end{array}$ & $\begin{array}{c}\text { Method of melatonin } \\
\text { assessment }\end{array}$ & Main findings & Limitations \\
\hline Grima et al. (2016) & $\begin{array}{l}\text { Individuals on } \\
\text { average six years } \\
\text { following severe } \\
\text { TBI reporting } \\
\text { symptoms of } \\
\text { chronic insomnia. } \\
\text { Patients with high } \\
\text { risk of sleep apnea } \\
\text { (based on } \\
\text { questionnaire } \\
\text { scores) were } \\
\text { excluded. }\end{array}$ & $\begin{array}{c}20 \\
\quad(10 \\
\text { patients } \\
\text { and } 10 \\
\text { healthy } \\
\text { controls })\end{array}$ & $\begin{array}{l}\text { DLMO test: salivary } \\
\text { melatonin } \\
\text { concentration was } \\
\text { determined from } \\
\text { samples taken from } \\
\text { seven hours prior } \\
\text { habitual bedtime to } \\
\text { two hours after } \\
\text { habitual wake time } \\
\text { under dim light } \\
\text { conditions. }\end{array}$ & $\begin{array}{l}\text { DLMO was, on average, } \\
1.5 \text { later in the TBI than in } \\
\text { the control group } \\
\text { The TBI group produced } \\
42 \% \text { less melatonin (based } \\
\text { on the area under the } \\
\text { curve) than the control } \\
\text { group. }\end{array}$ & $\begin{array}{l}\text { Actigraphy data was } \\
\text { not collected to } \\
\text { assess sleep-wake } \\
\text { schedule. } \\
\text { Only average group } \\
\text { DLMO was reported. } \\
\text { It would have been } \\
\text { interesting to know } \\
\text { from a clinical } \\
\text { perspective if there } \\
\text { were individuals who } \\
\text { had delayed/ } \\
\text { advanced DLMOs or } \\
\text { circadian sleep } \\
\text { disorders. Also, given } \\
\text { the sample } \\
\text { size/group, even a } \\
\text { few people with } \\
\text { extreme DLMOs } \\
\text { could have delayed } \\
\text { the mean DLMO. }\end{array}$ \\
\hline
\end{tabular}

Note: $\mathrm{TBI}=$ traumatic brain injury; DLMO $=$ dim light melatonin onset 


\begin{tabular}{|c|c|c|c|c|c|}
\hline Authors (year) & Study sample & $\begin{array}{c}\text { Sample } \\
\text { size }\end{array}$ & $\begin{array}{c}\text { Method of melatonin } \\
\text { assessment }\end{array}$ & Main findings & Limitations \\
\hline Ayalon et al. (2007) & $\begin{array}{l}\text { Sample was } \\
\text { selected from } 42 \\
\text { individuals with a } \\
\text { history of mTBI } \\
\text { who had insomnia } \\
\text { symptoms and also } \\
\text { reported other } \\
\text { symptoms of } \\
\text { circadian sleep- } \\
\text { wake disorders at } \\
\text { initial screening. }\end{array}$ & 15 & $\begin{array}{l}\text { DLMO test: salivary } \\
\text { melatonin } \\
\text { concentration was } \\
\text { determined from } \\
\text { samples taken in } \\
\text { every two hours for a } \\
\text { 24-hour period. }\end{array}$ & $\begin{array}{l}\text { Each of the } 15 \text { patients } \\
\text { were diagnosed with a } \\
\text { circadian sleep-wake } \\
\text { disorder: eight with } \\
\text { delayed phase sleep-wake } \\
\text { disorder and seven with } \\
\text { irregular sleep-wake } \\
\text { rhythm }\end{array}$ & $\begin{array}{l}\text { DLMO was not } \\
\text { reported. } \\
\text { Saliva samples were } \\
\text { taken in every two } \\
\text { hours instead of the } \\
\text { standard } 30 \text { min or } 60 \\
\text { minute intervals. } \\
\text { Only } 36 \% \text { of the } \\
\text { initial sample of } 42 \\
\text { patients received } \\
\text { circadian assessment. }\end{array}$ \\
\hline
\end{tabular}

Note: $\mathrm{TBI}=$ traumatic brain injury; DLMO = dim light melatonin on 
With respect to the chronic stage of severe TBI, rehabilitation patients and healthy controls had similar mean DLMOs (Shekleton et al., 2010; Steele et al., 2005). However, when patients with chronic insomnia were selected, their mean DLMO was approximately 1.5 hours later than the mean DLMO of the control group (Grima, Ponsford, St Hilaire, et al., 2016). This finding suggests that abnormal (i.e., decreased and/or delayed) melatonin production is associated with insomnia symptoms and may be prevalent among patients who experience insomnia in the chronic stage of severe TBI.

Insomnia is a shared symptom of CRSWD, including the ones caused by delayed melatonin production (Table 5.2). There is also evidence to suggest that healthy individuals with insomnia produce less melatonin than good sleeper controls (Riemann et al., 2002). It is not possible to discern if the findings of the above TBI studies simply reflect an increased prevalence of CRSWD and DLMO abnormalities among patients with insomnia or if there is a unique role of the brain injury. From a clinical perspective, it would be important to know if there is a high prevalence of CRSWD among patients who report insomnia symptoms following a TBI so as to make informed decisions about assessment and treatment. The above studies reported only mean DLMOs but did not describe what percentage of patients had a delayed DLMO or a CRSWD. Understanding what percentage of patients with insomnia has a CRSWD and exploring how abnormal melatonin production contributes to their insomnia would also be crucially important with respect to mTBI, given that insomnia symptoms are most frequently reported by individuals who had an mTBI but the clinical underpinnings of insomnia symptoms are not well explored in this population. 
Table 5.2.

Key Features of the Circadian Sleep-Wake Disorders Diagnosed in This Study

\begin{tabular}{|c|c|c|c|c|}
\hline Disorder & Sleep pattern & Main clinical features & Epidemiology & Treatment \\
\hline DSWPD & $\begin{array}{l}\text { Delayed main sleep } \\
\text { episode (usually by at } \\
\text { least two hours) } \\
\text { relative to the socially } \\
\text { desired or required } \\
\text { sleep time. }\end{array}$ & $\begin{array}{l}\text { Sleep initiation insomnia } \\
\text { and difficulty with waking } \\
\text { when sleep schedule is } \\
\text { socially imposed, but } \\
\text { significantly improved sleep } \\
\text { when sleep schedule is } \\
\text { freely chosen. } \\
\text { - Excessive sleepiness in the } \\
\text { morning. } \\
\text { Risk for the development of } \\
\text { mood disorders. }\end{array}$ & $\begin{array}{l}\text { Usually starts in } \\
\text { adolescence. } \\
\text { Prevalence in general } \\
\text { population: } 0.17 \%-1.53 \% \\
\text { - } 5-10 \% \text { of individuals with } \\
\text { chronic insomnia in sleep } \\
\text { clinics }\end{array}$ & $\begin{array}{l}\text { - Strategically } \\
\text { timed oral } \\
\text { melatonin based } \\
\text { on DLMO }{ }^{\mathrm{c}, \mathrm{ad}, \mathrm{a}} \\
\text { - } \\
\text { Post-awakening } \\
\text { light therapy in } \\
\text { conjunction with } \\
\text { behavioural } \\
\text { treatment }\end{array}$ \\
\hline ASWPD & $\begin{array}{l}\text { Advanced main sleep } \\
\text { episode (at least by two } \\
\text { hours) relative to the } \\
\text { socially desired or } \\
\text { required sleep time }\end{array}$ & $\begin{array}{l}\text { Inability to stay awake until } \\
\text { the socially desirable time } \\
\text { in the evening and inability } \\
\text { to remain asleep until a } \\
\text { socially desirable wake } \\
\text { time. } \\
\text { Excessive sleepiness in the } \\
\text { evening. }\end{array}$ & $\begin{array}{l}\text { Approximately } 1 \% \text { of the } \\
\text { general population has } \\
\text { advanced sleep phase } \\
\text { Prevalence of ASWPD in } \\
\text { the general population and } \\
\text { in sleep clinic insomnia } \\
\text { samples is unknown but } \\
\text { thought to be rare. }\end{array}$ & $\begin{array}{l}\text { - Evening light } \\
\text { therapy }\end{array}$ \\
\hline ISWRD & $\begin{array}{l}\text { Irregular sleep episodes } \\
\text { typically shorter than } 4 \\
\text { hours; total } 24 \text { h sleep } \\
\text { duration can be normal. }\end{array}$ & $\begin{array}{l}\text { Insomnia at night and excessive } \\
\text { sleepiness during the day. }\end{array}$ & $\begin{array}{l}\text { Described in neuro- } \\
\text { developmental and neuro- } \\
\text { degenerative disorders and } \\
\text { after TBI. }\end{array}$ & $\begin{array}{l}\text { Light therapy in } \\
\text { elderly patients } \\
\text { with dementia } \\
\text { - Melatonin c, ad }\end{array}$ \\
\hline
\end{tabular}

Note: DSWPD: Delayed Sleep-Wake Phase Disorder; ASWPD: Advanced Sleep-Wake Phase Disorder; IPSWD: Irregular Sleep-

Wake Rhythm Disorder; TBI: traumatic brain injury; DLMO: dim light melatonin onset; c: children; ad: adolescents; a: adults 
There has been only one study concerning melatonin assessment subsequent to mTBI (Ayalon et al., 2007). In that study, 15 individuals were selected from 42 patients who were referred for insomnia assessment to a sleep clinic (selection criteria were not reported). These 15 individuals received a circadian assessment, including actigraphy, 24-hour saliva melatonin and core body temperature measurement. Eight of the 15 patients were diagnosed with delayed sleepwake phase disorder (DSWPD) and the remaining seven received an irregular sleep-wake rhythm disorder (ISWRD) diagnosis; however, DLMOs were not reported for either sub-group.

Completing sleep diary, actigraphy and DLMO assessment for the whole group of 42 patients (instead of only for 15) and comparing participants with CRSWD to those without could have yielded interesting findings that could have shed light on the circadian aspect of insomnia symptoms in patients with and without a CRSWD. For example, it is possible that patients with post-concussion insomnia disorder have low melatonin secretion with normal DLMO; whereas the group with CRSWD may show the opposite pattern. It is also possible that there are different phase angles between DLMO and main sleep diary/actigraphy sleep parameters (e.g., sleep onset, sleep midpoint, and wake time) in the insomnia and the circadian disorder sub-groups, which has implications for DLMO estimations from sleep diary data. Further limitations of this study were that time since injury was not specified and the age range was restricted to young and early-middle age adults. The authors suggested that future studies should use larger samples and determine the DLMO among individuals with mTBI.

\section{The Objective and Hypothesis of the Current Study}

The second objective of the dissertation study was to replicate and extend the previous research by determining both the percentage of abnormal DLMO and the prevalence of CRSWD in a full sample of individuals reporting symptoms of chronic insomnia following mTBI. Based 
on previous research, the hypothesis was that rate of delayed DLMO and CRSWD would be higher in the mTBI sample than is reported for individuals who seek treatment for chronic insomnia in sleep clinics (10\%) (AASM, 2008).

\section{Method}

Participants. All participants of the dissertation study completed at least two components of the circadian assessment (sleep diary, actigraphy and DLMO test); please see dissertation sample characteristics in Table 3.2 and a descriptive summary of the sample in Chapter 3.

Missing data. Two participants had missing actigraphy data but both provided sleep diary data that could be used to determine if they had a normal sleep phase. Three participants did not have a DLMO assessment: one did not participate because she had low back pain and two participants did not show up on the scheduled date. Altogether, 47 of the 50 participants completed the DLMO test. DLMO could not be precisely determined from five samples. One of these was from a participant with a delayed sleep phase (according to his sleep history, sleep diary and actigraphy), whose melatonin did not start to rise during the test. The most parsimonious explanation is that his DLMO occurred approximately between 1 am and 1.30 am (based on his sleep pattern) after the last sample was taken. Two participants had high saliva melatonin concentrations from the first measurement; one clearly had advanced sleep phase and DLMO was probably missed even with an early first sample, whereas the other participant had normal sleep phase and the reason for high melatonin level is unknown. There were technical problems with two additional assays and the samples were not retained for re-analysis.

Procedures. As described earlier, the data for the circadian component of this dissertation were collected concurrently as part of the comprehensive assessment protocol (please see Figure 2.1 and Table 2.1). More specifically, individuals participated in a clinical interview and completed 
questionnaires at the Sleep and Alertness Clinic, Toronto, Canada. In the subsequent 12-14 days, they wore an actiwatch on their non-dominant wrist and they also kept the Consensus Sleep Diary during this period. Finally, they participated in a DLMO test 12-14 days following their initial interview.

Assessment tools and methods. The initial interview used the Duke Structured Sleep Interview and the SCID 5. Participants also completed a questionnaire booklet; the most relevant questionnaire to this chapter is the MEQ. Participants' sleep patterns were captured with the Consensus Sleep Diary and wrist actigraphy. Saliva melatonin samples were collected in the Melatonin Testing Laboratory, Toronto, Canada; for detailed description of these measures and procedures please see Appendix D.

\section{Analyses}

Saliva samples were analysed using enzyme-linked immunosorbent assay (ELISA) as per manufacturer's instructions (Buhlmann EK DSM (C) Saliva Melatonin ELISA kit; Buhlmann Laboratories, Switzerland). DLMO was determined according to consensus guidelines, see Appendix D (Benloucif et al., 2008).

Descriptive statistics summarized the sample characteristics, the sleep diary data (diary bedtime, sleep onset, sleep midpoint, wake time and rise time), the frequency of CRSWD diagnoses, the relationship between CRSWD diagnoses and demographic data and the correspondence between CRSWD and MEQ categories.

Pearson's correlations were used to quantify the direction and strength of relationship between MEQ, DLMO, and age as well as among DLMO and sleep diary bedtime, sleep onset, sleep midpoint, wake time and rise time. Independent-samples t tests compared if there was a statistically significant difference between patients without CRSWD and those diagnosed with 
DSWPD in sleep diary bedtime, sleep onset, sleep midpoint, wake time and rise time as well as the period elapsed between these sleep parameters and DLMO (Only the non CRSWD and the DSWPD groups were compared because the number of patients with other CRSWD was too small for inclusion into statistical group comparisons). Bonferroni corrections were applied to each statistical test to reduce family-wise error rates.

\section{Results}

Based on a compressive assessment, including clinical interview, sleep diary, actigraphy and DLMO, 13 individuals (26\% of the sample) received a circadian CRSWD diagnosis according to ISCD 3 criteria. The most common diagnosis (10 patients) was DSWPD; nine of these individuals were younger than 32 years. Two participants had advanced sleep-wake phase disorder (ASWPD) and one had ISWRD, these participants were older than 40. Additionally, six of 47 participants who completed a DLMO test had a DLMO outside of the normal time frame (five early and one delayed) without a CRSWD diagnosis. Altogether, $38 \%$ of the full sample (42\% of those who completed the DLMO test) had abnormal DLMO results.

The MEQ had a significant, positive correlation with age, $r=.48[.23, .70], p<.01$. This implies that the older the participants were the more indicative their MEQ scores were for morning circadian preference in accordance with findings in the general population (Paine, Gander, \& Travier, 2006). The MEQ was also significantly related to DLMO; specifically, there was a medium, negative correlation between these two variables, $r=-.36[-.61,-.07], p=.04$ showing that individuals reporting more morning circadian preference had earlier rise of saliva melatonin. However, the relationship between DLMO and MEQ was somewhat weaker and became statistically non-significant after controlling for age, $r=-.30[-.61, .09], p=.08$. 
The correspondence between the MEQ and the clinical diagnoses was the highest in patients with ASWRD and poorest in the DSWRD group (Table 5.3).

Table 5.3.

Relationship between Chronotype Categories and CRSWDs

\begin{tabular}{lccc}
\hline & $\begin{array}{c}\text { Morning Type } \\
(\mathbf{\%})\end{array}$ & $\begin{array}{c}\text { Intermediate type } \\
(\boldsymbol{\%})\end{array}$ & $\begin{array}{c}\text { Evening type } \\
(\boldsymbol{\%})\end{array}$ \\
\hline ASPD $(\mathrm{n}=2)$ & 100 & 0 & 0 \\
No circadian disorder $(\mathrm{n}=37)$ & 20 & 63 & 43 \\
DWSPD $(\mathrm{n}=10)$ & 0 & 57 & 43 \\
\hline
\end{tabular}

Note: The table shows the percentage of participants without a circadian disorder; the percentage of participants with delayed phase disorder (DSWPD) and the percentage of participants with advanced sleep-wake phase disorder (ASWPD) whose scores were in the intermediate, evening and morning type categories on the Morningness-Eveningness Scale.

There were large and significant differences both in the subjective behavioural (sleep diary) and objective, biological (DLMO) timing of sleep and circadian rhythm between patients with DSWRD and those without a CRSWD, confirming that the clinical diagnoses accurately separated patients with DSWRD from those without a circadian disorder (Table 5.4).

The comparison of differences (phase angles) between DLMO and sleep diary sleep times showed that patients with DSWPD fell asleep 48 minutes closer to their DLMO and woke 42 minutes further away from their DLMO than patients with insomnia disorder. These differences have medium effect sizes and may be clinically significant despite lacking statistical significance $(t=1.93(35), p=.06, r=.31$ for sleep onset and $t(7.6)=-0.86, p=.18, r=.29$ for wake time).

With regards to the correlations between DLMO and sleep diary sleep timing, there were strong and statistically significant bivariate correlations between DLMO and bedtime, sleep onset, sleep midpoint, time of final awakening and rise time in the sample; however, this was due 
to the strong collinearity among the sleep diary variables. When the unique relationships between the individual sleep variables and the DLMO were examined, the correlations were nonsignificant in the whole sample as well as in the insomnia disorder group and the DSWPD groups separately (Table 5.5.). However, the effect sizes suggest that the sleep phase of the DSWPD group was more in synchrony with their internal time than the sleep phase of the insomnia disorder group. 
Table 5.4.

Comparison of DLMO and Sleep Timing between Patients Diagnosed with Insomnia Disorder and Delayed Phase Sleep-Wake Disorder

\begin{tabular}{|c|c|c|c|c|}
\hline & \multicolumn{2}{|c|}{$\begin{array}{c}\text { Insomnia Disorder } \\
(N=24)\end{array}$} & \multicolumn{2}{|c|}{$\begin{array}{l}\text { Delayed Phase Sleep-Wake Disorder } \\
\qquad(N=10)\end{array}$} \\
\hline & Mean $(S D)$ & $\begin{array}{l}95 \% \mathrm{BCa} C I \text { of } \\
\text { the Mean }\end{array}$ & Mean $(S D)$ & $\begin{array}{l}95 \% \mathrm{BCaCI} \text { of } \\
\text { the Mean }\end{array}$ \\
\hline DLMO (hours: minutes) & $20: 24(1.8)^{*}$ & $20: 06-20: 40$ & $22: 73(0.63)^{*}$ & $22: 18-23: 18$ \\
\hline Bedime (hours: minutes) & $23: 34(0.8)^{*}$ & $23: 17-23: 50$ & $24: 55(1.0) *$ & $24: 19-25: 40$ \\
\hline Sleep onset time (hours: minutes) & 24: $17(0.9)^{*}$ & $24: 01-24: 39$ & 02:00 $(1.0)^{*}$ & $01: 26-02: 36$ \\
\hline Sleep midpoint (hours: minutes) & $03: 50(.53)^{*}$ & 03:41-04:03 & $05: 20(1.3)^{*}$ & $04: 32-06: 06$ \\
\hline Wake time (hours: minutes) & $7: 21(0.5)^{*}$ & $7: 14-7: 34$ & $8: 41(1.9)^{*}$ & $7: 30-9: 45$ \\
\hline Getting out of bed time (hours: minutes) & $8: 02(0.6)^{*}$ & $7: 53-8.18$ & $9: 41(1.8)^{*}$ & $8: 29-10: 47$ \\
\hline DLMO - bedtime (hours) & $3.3(1.3)$ & $2.8-3.8$ & $3.1(1.1)$ & $2.0-4.0$ \\
\hline DLMO - sleep onset (hours) & $4.0(0.9)$ & $3.6-4.4$ & $3.2(1)$ & $2.4-4.0$ \\
\hline DLMO - sleep midpoint (hours) & $7.5(0.8)$ & $7.2-7.8$ & $6.8(1.4)$ & $5.6-7.9$ \\
\hline DLMO - wake time (hours) & $13.0(0.8)$ & $13.0-13.4$ & $13.6(1.8)$ & $12.3-15.2$ \\
\hline DLMO - getting out of bed time (hours) & $12.3(0.9)$ & $12.0-12.6$ & $12.7(2.1)$ & $11.3-14.2$ \\
\hline
\end{tabular}

Note: $95 \mathrm{BCa} \mathrm{CI}=95 \%$ bias corrected accelerated confidence interval based on 1000 bootstrapping samples. * denotes statistically significant differences between the two groups. Time is in the International Organization for Standardization (ISO) 8601 format 
Table 5.5.

Correlations between DLMO and Sleep Diary Sleep Timing Variables

\begin{tabular}{lccccc}
\hline & Lights Out & Sleep Onset & Sleep Midpoint & $\begin{array}{c}\text { Final } \\
\text { Awakening }\end{array}$ & $\begin{array}{c}\text { Rise time } \\
\text { Full Sample }\end{array}$ \\
Insomnia Disorder & $.02[-.29 ; .30]$ & $-.38[-.71 ; .13]$ & $.36[-.24 ; .64]$ & $-.36[-.72 ; .15]$ & $.16[-.10 ; .40]$ \\
DSWPD & $-.25[-.72 ; .18]$ & $-.08[-.50 ; .17]$ & $-.09[-.56 ; .84]$ & $-.08[-.52 ; .18]$ & $.30[-.27 ; .60]$ \\
\hline
\end{tabular}

Note. The table shows the partial correlation coefficients for the unique relationship between the Dim Light Melatonin Onset (DLMO) and sleep diary sleep timing variables (with the other four sleep timing variables partialed out). Bootstrap bias $95 \%$ corrected and accelerated confidence intervals for the correlation coefficients are shown in brackets. None of the correlations were statistically significant after Bonferroni corrections were applied. 


\section{Discussion}

The second objective of this dissertation and the focus of the present chapter was to determine what percentage of patients who develop chronic insomnia following a mTBI have a CRSWD or abnormal timing of DLMO that might contribute to their insomnia symptoms. Following a comprehensive assessment of the circadian timing of sleep and wakefulness, $26 \%$ of the sample received a CRSWD diagnosis and $42 \%$ of patients who participated in a DLMO test had a DLMO outside of the normal clock time frame.

Delayed Sleep - Wake Phase Disorder related findings. Most (77\%) of the participants who received a CRSWD diagnosis had DSWPD. The percentage of patients with DSWPD in the current study (20\%) is almost identical to the one described in the previous study (19\%) concerning circadian phase sleep-wake disorders among patients with insomnia following mTBI with the caveat the in the previous study only 15 patients were provided with a circadian assessment (Ayalon et al., 2007). This proportion is one-two orders of magnitude higher than the prevalence of DSWPD in the general population $(0.17 \%-1.53 \%)$ and over three times that among patients with chronic insomnia treated at sleep clinics $(6.7 \%)$ (Paine, Fink, Gander, \& Warman, 2014; Schrader, Bovim, \& Sand, 1993; Weitzman et al., 1981).

One could argue that DSWPD is more common among patients with mTBI than in general sleep clinic insomnia samples because they are younger than patients with insomnia who are treated at sleep clinics. Indeed, the mean age was 26 years in the previous mTBI circadian study and each participant (except for one individual) diagnosed with DSWPD in the current study was an adolescent or a young adult. However, the estimated prevalence of DSWPD is between $3.3-7.3 \%$ in the adolescent population and between $7 \%$ and $16 \%$ in clinical samples of this age group (AASM, 2014; Lovato, Gradisar, Short, Dohnt, \& Micic, 2013; Saxvig, Pallesen, 
Wilhelmsen-Langeland, Molde, \& Bjorvatn, 2012; Sivertsen et al., 2013). In contrast, 56\% of patients $\leq 31$ years of age had DSWPD in the current study. Based on these results, we can conclude that the prevalence of DSWPD is approximately three-fold among patients with insomnia and mTBI compared to general sleep clinic insomnia samples and it is particularly high (approximately five-fold) among adolescents and young adults who seek assessment for insomnia following a mTBI.

This study did not explore the causes of DSWPD but several etiological factors merit consideration. One possibility is that the injury impacted the anatomical pathways involved in the circadian timing of sleep and wakefulness. One study noted that patients who reported having sleep problems following a mTBI had a longer tentorium (measured from the posterior-inferior margin of the torcula to its anterior termination on the MRI) and a flatter tentorial angle (formed by the McRae line through the foramen magnum and a line drawn along the length of the tentorium) than patients who did not have sleep problems. The authors speculated that the pineal gland would be impacted by injuries caused by an anterior - posterior force in individuals with this anatomical predisposition (Yaeger, Alhilali, \& Fakhran, 2014).

Another biological predisposing factor could be a susceptibility of the sleep and circadian system for phase delay. Population-based studies show that symptoms of phase delay are relatively common compared to the prevalence of the DSWPD, as a clinical disorder (Lovato et al., 2013; Saxvig et al., 2012; Sivertsen et al., 2013). A possible explanation for the high frequency of DSWPD among patients with insomnia following a mTBI is that these individuals had a circadian predisposition for phase delay or perhaps even had symptoms of this disorder before the injury but the full syndrome manifested only in the post-injury period. 
Indeed, at least half of the patients with DSWPD in the current study reported that they could wake up early to attend sport training or school or work in the morning but they preferred late bedtimes and late wake times when they were free to choose their sleep schedule, for example on the weekends or when they were on vacation. This evening preference may have had a biological underpinning - for example a long circadian period, heightened responsiveness to the melatonin supressing effect of light in the evening; or slow accumulation and dissipation of homeostatic sleep drive - mechanisms that have been implicated in the etiology of DSWPD (Micic et al., 2016).

In the post-injury period, when there is a gradual return to pre-injury activities, early morning activities may not be scheduled. During this period the sleep phase can follow the circadian signals (rather than being set by social demands) and naturally shifts to later hours in individuals with a biological predisposition for phase delay. This can lead to a vicious cycle, in which longer light exposure in the evening and lack of light exposure in the morning further delays the circadian cycle (Burgess \& Eastman, 2006). Additionally, the plummeting physical activity level and the opportunity to sleep longer in the morning may decrease the homeostatic sleep drive, which, in turn, decreases sleepiness in the evening and can contribute to the delay of the sleep period. Finally, it has been shown that DSWPD has an insomnia component, including psycho-physiological arousal, which also delays sleep onset. Patients with DSWPD in the current sample had high scores on cognitive insomnia questionnaires, suggesting that psychological factors also contributed to their sleep initiation difficulty. Ultimately, the combination of biological, social and psychological factors may lead to the development of DSWPD among individuals with a biological predisposition for phase delay. Once the DSWPD develops, it can 
become an obstacle to resuming the pre-injury activity level and this, in turn, can perpetuate the sleep and circadian problem.

It is also important to note that having a pre-injury delayed DLMO could increase the risk for sustaining a TBI. Individuals with a late DLMO and evening chronotype are sleepy when they get up early for sport training, school or work. Athletic performance fluctuates across the circadian cycle and excessive sleepiness impacts cognitive performance and increases the risk for accidents and injuries (Atkinson \& Reilly, 1996; Bioulac et al., 2017; Dewald, Meijer, Oort, Kerkhof, \& Bogels, 2010; C. L. Drake et al., 2010; Melamed \& Oksenberg, 2002; Milewski et al., 2014; Silva, Wang, Ronda, Wyatt, \& Duffy, 2010). Thus, the high prevalence of DSWPD in mTBI samples may, in part reflect a higher prevalence of TBI among individuals with a late DLMO and evening chronotype.

It is important to emphasize that close to half of the DSWPD group had scores on the MEQ in the intermediate chronotype range and approximately one third of patients with DSWPD reportedly had an intermediate sleep schedule even when they were free of social demands. These patients less likely had a circadian predisposition for DSWPD and other factors may have played a relatively larger role in the development of DSWPD.

\section{Advanced Sleep-Wake Phase Disorder and Irregular Rhythm Sleep - Wake Disorder} related findings. Two participants received a diagnosis of ASWPD in the present study. An additional five participants had an advanced DLMO but did not receive a diagnosis of ASWPD because: (1) the early sleep phase did not cause distress or functional impairment; (2) they were diagnosed with OSA or PLMD that could also explain the early morning awakenings or (3) their sleep phase was not advanced. The prevalence of ASWPD is not known in the general population or in insomnia clinical samples but is estimated to be rare, given that only individual 
cases and family cohorts have been described thus far (AASM, 2014; Schrader et al., 1993). The previous circadian study did not identify any patients with ASWPD in the mTBI insomnia sample (Ayalon et al., 2007). One possible reason for this is that those patients were not selected for circadian assessment. Another possibility is this disorder is as rare in this clinical group as it is in the general population. A third explanation is that the previous mTBI insomnia sample consisted of young and middle age adults (the mean age was 26 years and the maximum age was 45 years). Sleep phase normally advances with age and it is reasonable to assume that ASWPD is more common among older than younger adults who suffer from subjective symptoms of insomnia (Paine et al., 2014). In the current study, the two patients with ASWPD that we identified were older than 45 . One of them reported that the early evening sleepiness and early morning final awakening started shortly after the head injury. The other participant described having a normal sleep phase until approximately four years before the head injury. At the time, the participant moved to the countryside and adopted a new lifestyle that included going to bed and waking up early. This sleep phase further advanced following the injury which caused significant distress for the individual. There was no family history of early sleep phase in either case based on the information provided by the participants.

Finally, one participant in the present study received the diagnosis of ISWRD. This circadian sleep-phase disorder has been described among individuals with brain pathology, including neurodevelopmental or neurodegenerative disorders and traumatic brain injury (AASM, 2014). In the previous circadian study, 16 percent of the participants were diagnosed with ISWRD - eight times more than in the present study (Ayalon et al., 2007). The reason for this substantial difference cannot be easily explained. One possibility is that patients in the previous study were assessed sooner after the injury, when their sleep schedule was more 
disrupted, including daytime naps and more significant insomnia at night. Unfortunately, the information about the time elapsed since the injury was not provided in the previous publication. One can also speculate that a complicated mTBI or perhaps a brain pathology pre-dating the injury increases the risk for ISWRD. The person in the present study who received an ISWRD diagnosis reportedly had had alcohol dependence until six months before his injury. There is an interaction between the circadian system and alcohol use disorders: circadian disruption increases the risk for developing alcohol use disorders and alcohol directly impacts the function of the central pacemaker- both its internal rhythm and its response to light (Rosenwasser, 2015). The cause of the ISWRD cannot be identified in the current study but it is possible that the patient had a pre-existing circadian disruption related to his alcohol dependence that increased his risk for developing an ISWRD following the injury. The mTBI may have played causal or additive, direct or indirect role in the evolution of his sleep disorder.

The relationship between sleep diary and DLMO data. In this study we collected sleep diary, actigraphy and DLMO data from the full sample not only to avoid inaccurate diagnoses but also to investigate if a misalignment between the DLMO and sleep phase could contribute to insomnia symptoms in patients with and without a CRSWD diagnosis. One can hypothesize that a too short or a too long phase angle between the timing of sleep and DLMO can contribute to insomnia symptoms. For example, if bedtime is too close to the DLMO, the person may have a sleep initiation difficulty unless the homeostatic sleep drive is strong (for a description of the interaction between the homeostatic and circadian systems, please see Appendix E). Conversely, if the phase angle between DLMO and sleep phase is too wide -i.e., bedtime is late compared to the DLMO- the circadian awakening signals will shorten sleep duration and may cause subjective symptoms of sleep maintenance insomnia or early final awakening. 
The findings of this study show that the average difference between DLMO and bedtime was approximately 30 minutes longer and the average period between DLMO and sleep onset was approximately one hour longer in participants with insomnia disorder than previously published values for good sleepers (Burgess \& Eastman, 2005). The time lapse between the DLMO and sleep onset in the current study was the same as in a previous study involving patients with sleep onset insomnia (Wright, Lack, \& Bootzin, 2006). In contrast to the elongated DLMO-sleep onset period, the difference between wake time and DLMO in the insomnia disorder group was the same as it is in good sleepers (Burgess \& Eastman, 2005). This implies that the awakening signals from the circadian system woke the participants in their biological morning even though they fell asleep late in their biological night. The sleep phase of patients with DSWPD were better aligned with their DLMO and the phase angle between DLMO and sleep onset was similar to that of good sleepers, which replicates the results of a previous study (Wright et al., 2006). However, the variation of the wake time and the DLMO-wake time phase angle was more than 1 SD larger in the DSWPD than in the insomnia disorder group, suggesting that some patients with DSWPD could sleep in their biological sleep window (i.e. they had a late wake up time) while others had to wake up relatively early relative to their internal time.

Collectively, the above observations suggest that the circadian system contributed to the insomnia symptoms differently in the insomnia disorder versus in the DSWPD groups. In patients who did not have a CRSWD, the sleep onset difficulty could not be explained by a too early bedtime relative to the DLMO. Instead, other - most likely psychological factors accounted for their sleep initiation difficulty. With regards to wake times in the insomnia group, the otherwise normally entrained circadian system may have contributed to their sleep 
maintenance difficulty by sending awakening signals when they tried to extend their sleep to compensate for the "lost" hours of sleep at night.

As opposed to patients with insomnia disorder, participants with DSWPD fell asleep later than they desired because of their delayed DLMO. In contrast to the patients with normally entrained circadian system, their sleep duration was not curtailed by the wakening signals of the circadian system; rather it may have been constrained by social demands.

Clinical implications. The findings of this study have important implications for the assessment and treatment of post concussion chronic insomnia. With regards to assessment, clinicians should collect information about pre-injury preferred sleep schedule and the change in the timing of sleep since the brain injury. Referral to a sleep specialist for a circadian assessment is warranted if there is a significant shift of the sleep period to an advanced or delayed regular sleep onset and wake time or if the patient sleeps only for short (maximum 4 hours) periods around the clock. The threshold for conducting a circadian assessment should be especially low if a teenager or a young adult reports significant sleep onset insomnia or significant delay of sleep phase following a mTBI. Actigraphy monitoring - ideally for two consecutive weeks - is recommended for the assessment of sleep-wake schedule to inform diagnosis; if actigraphy is not available, patients should be provided with a sleep diary to collect prospective information about sleep (Auger et al., 2015).

The actigraphy assessment is ideally followed by a DLMO test if the actogram shows an abnormal sleep-wake pattern. Though not required by the clinical guidelines, conducting DLMO test provides important information for diagnosis and treatment planning (Keijzer, Smits, Duffy, \& Curfs, 2014; Morgenthaler et al., 2007). With regards to its diagnostic utility, DLMO is the best biological marker of the circadian phase; thus, determining the DLMO helps to clarify if the 
abnormal sleep schedule has a biological underpinning (Rahman, Kayumov, Tchmoutina, \& Shapiro, 2009). Another advantage of conducting a DLMO assessment is that it aids treatment planning. The first line evidence-based treatment for DSWPD is low dose melatonin taken during the phase advance part of the circadian melatonin phase response curve (Auger et al., 2015). Specifically, patients should be instructed to take $0.5 \mathrm{mg}$ melatonin 2.5 hours prior to their DLMO to achieve a maximum phase advance with a minimum hypnotic effect (Burgess \& Emens, 2016; Burgess, Revell, \& Eastman, 2008; Lewy, Emens, Sack, Hasler, \& Bernert, 2002). In contrast, administering melatonin 10 hours after the DLMO has a phase advance effect (Lewy, Ahmed, Jackson, \& Sack, 1992). Thus, knowing precisely when the DLMO occurs helps to plan individually-tailored treatment.

Unfortunately, DLMO testing is expensive and DLMO testing laboratories are scarce. Home-based saliva collection is cheaper and produces comparable results to in-lab DLMO tests but it still relies on laboratories to conduct the assays (Burgess, Park, Wyatt, \& Fogg, 2016; Burgess, Wyatt, Park, \& Fogg, 2015). MEQ scores correlated with the DLMO in this study and in previous research but the DLMO cannot be precisely calculated from these questionnaire scores (Kantermann, Sung, \& Burgess, 2015). Notably, the agreement between DLMO and chronotype categories was the poorest among participants who received a DSWPD diagnosis in this study - which is exactly the clinical group where we need to gauge DLMO accurately. A recent study showed that a combination of sleep diary sleep timing parameters accounted for $77 \%$ of variance in DLMO and could be used to predict the DLMO within 1.5 hours precision using regression models in 79\% of patients with DSWPD (Lovato et al., 2016). Regression could also predict DLMO with one-hour precision in 74\% of good sleepers (Burgess 
\& Eastman, 2005). Although these are large effects, clinicians need a simpler method than using a regression to estimate DLMO from sleep diary data, if DLMO testing is not available.

The general practice of estimating DLMO from bedtime or sleep onset may lead to an error in working with patients with insomnia disorder, especially ones with sleep onset insomnia. The results of this study, in accordance with a previous study suggest that the time lapse between the DLMO and perceived sleep onset is longer than normal among people with insomnia disorder (Wright et al., 2006). Specifically, DLMO occurs approximately four hours prior to subjective sleep onset - as opposed the 2.5 hours described in good sleepers. Since patients with insomnia disorder may have a varying degree of sleep onset difficulty and sleep misperception, using a general guideline to estimate DLMO from sleep diary sleep onset may be misleading. Instead, wake time appears to be a better point to anchor DLMO calculations in patients with insomnia disorder, since insomnia patients woke approximately 13 hours before their DLMO, as good sleepers do. In the current sample, DLMO could be estimated with 1.5 hours precision in $84 \%$ of the sample by adding 13 hours to sleep diary wake time. When the same method was employed in the insomnia disorder group and the DSWPD groups separately, DLMO could be estimated within 1.5 hours precision in $91 \%$ of the insomnia disorder group and in $75 \%$ of the DSWPD group; the latter was probably less accurate because of the larger variations in the phase angle between DLMO and wake time. It is important to note that the sample size for DSWPD was small and further studies with larger sample sizes will be required to provide further information with regards to the phase angles in patients who develop DSWPD following a mTBI.

In conclusion, one-quarter of patients referred for a post mTBI insomnia assessment had a CRSWD. The most common diagnosis was DSWPD, with a three-fold prevalence compared to 
sleep clinic insomnia samples. Based on these results, clinicians should recognise the symptoms of CRSWD and initiate a circadian assessment when patients with chronic insomnia report a shift of sleep phase following a mTBI.

In comparing patients with insomnia disorder to individuals with DSWPD, the sleep phase of patients with insomnia disorder was more out of synchrony compared to their internal time than the sleep phase of patients with DSWPD. The phase angle between DLMO and sleep onset was increased in patients with insomnia disorder compared to previously published good sleeper data, while the phase angle of waketime was normal. This has implications for estimating DLMO from sleep diary data, when DLMO testing is not available. A simple method of adding 13 hours to sleep diary wake time could be a useful for estimating DLMO, but this method appears to be more accurate in patients with post-concussion insomnia disorder than in patients with post-concussion DSWPD because the larger variations in final wake time among patients with DSWPD following a mTBI. 


\section{Chapter 6: Testing the REM Instability Hypothesis in Insomnia Following a Mild Traumatic Brain Injury}

Chronic insomnia in adults is regarded to be an essentially subjective experience of persistent sleep difficulty and the daytime consequences arising from this perception (AASM, 2014). Individuals - both good sleepers and patients with insomnia disorder - may over or underestimate their sleep duration and their wake time but the overestimation of wake time and underestimation of sleep time is more common among patients with insomnia disorder than among good sleepers (Means, Edinger, Glenn, \& Fins, 2003b). While individual studies may not show a significant difference in the PSG recordings of insomnia patients and good sleepers, the pooled data of more than 1000 participants from 23 studies showed that the SOL of patients with primary insomnia ${ }^{15}$ was six minutes longer than the SOL of good sleeper controls and patients with insomnia disorder spent 20 minutes more awake after their sleep onset than good sleepers did when these parameters were precisely measured in the sleep laboratory (Baglioni et al., 2013). Furthermore, they had six more awakenings per night than controls did. Subjective sleep diary data showed a more substantial discrepancy: patients with insomnia overestimated their SOL and WASO by approximately 20 minutes and 50 minutes, respectively. The results of this meta-analysis show that despite the heterogeneity in their accuracy of sleep perception, patients with primary insomnia disorder, as a group, have objectively detectable sleep continuity problems and at the same time, their perception of sleep impairment is magnified relative to the objective findings.

Objective differences in sleep architecture between primary insomnia patients and good sleepers are even more subtle than differences in their sleep continuity indices. Overall, patients

\footnotetext{
${ }^{15}$ Primary insomnia is a diagnostic category used in previous version of the DSM (DSM-IV). It referred to insomnia that was not caused by a medical (including psychiatric) condition.
} 
with primary insomnia had an approximately two percent reduction of both SWS and REM sleep per night compared to good sleepers (Baglioni et al., 2013). However small these differences appear to be, they amount to a substantial deficit of 600 minutes of SWS and 300 minutes of REM sleep per month. When individuals are effectively deprived of these two key sleep components, they may experience memory problems and mood instability, which may appear to be disproportionately severe relative to the objective reduction of their TST during the night. Based on these findings, it has been proposed that changes in SWS and in REM sleep may be related to the psychopathology of insomnia disorder (Baglioni et al., 2013).

The objective sleep abnormalities in TBI samples are similar to what has been described in primary insomnia. Based on a meta-analysis, patients with TBI have longer WASO, reduced TST and lower SE than controls and these differences are independent of the severity of the TBI (Grima, Ponsford, Rajaratnam, Mansfield, \& Pase, 2016). Furthermore, the sleep architecture of patients with mTBI showed reduced percentage of REM sleep compared to controls, while this difference was not present in studies involving patients with a history of moderate/severe TBI. As in primary insomnia, the magnitude of objective differences has been small compared to the degree of sleep problems patients report as per subjective sleep assessment tools (Grima, Ponsford, Rajaratnam, et al., 2016). In contrast to what has been described in primary insomnia, no difference has been detected in the amount of SWS between TBI and good sleeper groups (Grima, Ponsford, Rajaratnam, et al., 2016).

The objective sleep abnormalities in TBI samples may be caused by sleep disorders; however, the specific contribution of sleep disorders cannot be determined because in most studies sleep disorders were not diagnosed. Given that the same pattern of changes has been shown in sleep (mis)perception, objective WASO, SE, and REM sleep in mTBI and primary 
insomnia, a plausible possibility is that the difference of sleep between the mTBI and control samples was driven by individuals who had suffered from insomnia following the TBI.

Since sleep misperception is a characteristic feature of insomnia disorder and REM sleep has been proposed to play a role in its pathophysiology, combined with the fact that REM sleep is also affected in mTBI, it is plausible to test theoretical models of sleep misperception that focus on REM sleep in a clinical group of patients with both mTBI and chronic insomnia. The REM sleep instability hypothesis of insomnia posits that worry about sleep leads to short awakenings (i.e. micro arousals) from sleep and, in particular, from REM sleep (Riemann et al., 2012). During micro-awakenings from REM sleep, the individual becomes cognizant of anxious dream content, which resembles daytime and evening sleep-focused anxious thoughts. Consequently, REM sleep fragmentation blurs the distinction between sleep and wakefulness and causes a false perception of being awake while the individual is, in fact, asleep.

The REM sleep instability hypothesis is based on a study in which the AI was observed to be significantly higher in a group of 100 medication-free individuals with primary insomnia as compared to a control group of 100 good sleepers (Feige et al., 2008). Furthermore, subjective wake time during the night was predicted by both objective PSG wake time and the time spent in REM sleep. The authors' interpretation of this finding was that individuals with primary insomnia perceived and remembered REM sleep as time spent awake.

Though AI in REM sleep was higher among insomnia patients than in controls in the above study, the number of arousals was not a significant, independent predictor of subjective wake time above and beyond REM duration. If the REM instability hypothesis is correct, there may be a variable other than the number or rate of micro-arousals that corresponds with a (mis)perception of REM sleep as wakefulness in this clinical group. 
REM sleep represents a "third state" state of consciousness that is uniquely different from both wakefulness and deep sleep. During wakefulness, the level of consciousness (also conceptualized as arousal or vigilance) is high and the content of consciousness is shaped by awareness to the environment. The opposite pattern characterizes deep sleep - the level of consciousness and awareness to the environment are both decreased compared to the wake state. REM sleep is unique in that the awareness to the environment is decreased (as in deep sleep) but there is heightened level of consciousness (as in wakefulness), which, on the neurological level corresponds to an extended functional connectivity of the default mode network (DMN) compared to the wake state (Chow et al., 2013).

In line with the REM instability hypothesis, it is possible that chronic insomnia is associated with a unique pattern of consciousness in REM sleep; specifically, with a heightened level of consciousness/arousal and an increased awareness to the dream content which reflects daytime thoughts and experiences tinted with negative valence (Feige et al., 2008). This pattern could contribute to the misperception of REM sleep as wakefulness in patients with chronic insomnia.

\section{Objective and Hypothesis}

The third objective of the dissertation (and the focus of the current chapter) was to test and extend the previous research concerning the REM instability hypothesis of chronic insomnia in a group of patients with both mTBI and chronic insomnia. As per the original theory, the hypothesis was that subjective (sleep diary) wake time would be significantly correlated with PSG wake time and with time participants spend in REM sleep. As an extension of the theory, it was also proposed that subjective wake time would be associated with level of consciousness during REM sleep. 


\section{Method}

Participants. This dissertation component involved the same participants who took part in the larger dissertation study; please see Table 3.1 and a descriptive summary of the sample in Chapter 3.

Procedures (relevant highlights). The study protocol included three nights of PSG recording: one night in the laboratory and two nights in the participants' homes. The laboratory night was recorded 6 or 7 days after the clinical interview at the Sleep and Alertness Clinic in Toronto (Figure and Table 2.1). After the laboratory night, there was an unrecorded night, which was followed by the first home PSG and then the second home PSG. Participants also kept a Consensus Sleep Diary for the duration of the study, where they recorded their perceived sleep onset latency, time spent awake during the night and terminal wakefulness among other subjective sleep parameters.

Apparatus and measures. The description of the relevant assessment tools and methods, including the laboratory and home PSG as well as the sleep diary can be found in Appendix D. Analyses. Non-parametric Friedman's ANOVA (for non-normally distributed variables) and repeated measures ANOVAs compared the values of the predictor and outcome variables between the laboratory and home nights. The sleep diary (subjective) and the PSG (objective) wake times were compared using t tests. Bivariate correlation analyses quantified the relationship between the predictor and outcome variables. Bootstrapping was used in each analysis to obtain bias corrected and accelerated confidence intervals. For non-normally distributed variables both the robust parametric test statistics based on 2000 bootstrapping samples and the non-parametric test results are reported, if there was a disagreement between these tests either in significance level or effect sizes. Only the robust test results are reported 
when there was an agreement between the robust and the non-parametric tests both in terms of significance and effect size. The analyses were first conducted in the full sample and subsequently were repeated in the sub-group diagnosed with insomnia disorder.

\section{Results}

Objective PSG wake time (i.e., TIB - TST) was similar across the three recorded nights, $F(2,60)=0.85, p=.43, \eta^{2}=.03$ (Table 6.1 ). Likewise, there were no statistically significant differences between the subjective sleep diary wake times across these three nights $F(2,62)=$ $0.32, p=.72, \eta^{2}=.01$. However, when the subjective (sleep diary) and objective (PSG) wake times were compared to each other, a consistent pattern emerged. Specifically, patients overestimated the time they spent awake during the night compared to the objective PSG measure of wake time and these differences were statistically significant with a medium effect size both in the laboratory (mean difference: 31 minutes, BCa 95\% CI: $6.11-55.83, t(40)=$ 2.52, $p=.02, r=.37$ ) and at home (mean difference: 44 minutes, BCa 95\% CI: $9.34-78.78, t$ $(23)=2.62, p=.02, r=.47)$. 
Table 6.1.

Descriptive Statistics of Proposed Predictor and Outcome Variables - REM Instability Hypothesis

\section{LABORATORY NIGHT}

\begin{tabular}{|c|c|c|c|c|c|}
\hline & Mean (SD) & $\begin{array}{c}95 \% \text { CI of the } \\
\text { Mean }\end{array}$ & Median & $\begin{array}{l}\text { BCa } 95 \% \text { CI of } \\
\text { the Median }\end{array}$ & IR \\
\hline Diary Wake Time & $116.65(61.50)$ & $98.36-134.83$ & 109.80 & $94.80-135.07$ & 75.00 \\
\hline PSG Wake Time & & & 68.00 & $49.70-93.90$ & 74.80 \\
\hline Lab REM sleep duration (minutes) & $58.21(34.86)$ & $48.61-67.31$ & 66.00 & $37.50-72.00$ & 75.25 \\
\hline Relative LC & & & 0.0080 & $-0.0010-0.0370$ & 0.05 \\
\hline \multicolumn{6}{|c|}{ HOME NIGHTS } \\
\hline Diary Wake Time & $139.39(75.16)$ & $115.64-165.50$ & 118.65 & $104.70-165.00$ & 131.85 \\
\hline PSG Wake Time & $109.15(81.98)$ & $82.64-137.43$ & 82.60 & $58.97-126.65$ & 140.56 \\
\hline REM sleep duration (minutes) & & & 82.00 & $60.63-88.25$ & 65.94 \\
\hline Relative LC & 0.0197 & $0.0093-0.0306$ & 0.0123 & $0.0013-0.0375$ & 0.04 \\
\hline
\end{tabular}

Note: Wake times are calculated as time in bed minus total sleep time in minutes. Mean values are shown only for normally distributed variables 
The median AI in the full sample was normal both in the laboratory and at home (Table 6.2). Since OSA causes arousals from sleep, the AI of participants with OSA was compared to the AI of participants without OSA. Participants with OSA had significantly higher AI than participants who did not have OSA when they slept in the laboratory $(t(33.59)=2.99, p<.01, r$ $=.46)$ but not during the nights recorded at home $(p=.22, r=.20$ and second night home, $p=$ $.37, r=.22$ ), see Table 6.2 .

The overall difference in AI between the lab and home nights approached statistical significance on the omnibus non-parametric test, Friedman's $\chi^{2}(2)=5.63, p=.06$ and was statistically significant using ANOVA, $F(2,64)=5.08, p=.01, \eta^{2}=.14$ in the sample. Based on both the post-hoc Wilcoxon signed-ranked test and paired-samples t test with 2000 resamples, this difference was explained by significantly fewer arousals in the second night at home than in the laboratory, $\mathrm{Z}=-3.19, p<.01, r=.51$ or $t(38)=3.3, p<.01, r=.67$, which translates to a large effect. In contrast, there was no significant difference in the arousal indices between the laboratory and first night home recordings confirmed by both the non-parametric and the robust, parametric tests, $\mathrm{Z}=0.96, p=.33, r=.15$ and $t(37)=0.99, p=.33, r=.16$. Although participants had more arousals in the first than in the second night at home, this difference was small and statistically non-significant after Bonferroni corrections were applied, $\mathrm{Z}=1.46, p=$ $0.15, r=.23$ and $t(32)=2, p=.05, r=.34$. The greater AI among patients with OSA did not account for the difference between the laboratory and home AI, F OSA x location $(1,10)=0.67, p=$ $.42)$. 
Table 6.2.

Arousal Index Descriptive Statistics

\begin{tabular}{lcccccc}
\hline & \multicolumn{2}{c}{ Full Sample $(N=\mathbf{5 0})$} & $\begin{array}{c}\text { Participants with OSA } \\
(N=\mathbf{2 2})\end{array}$ & $\begin{array}{c}\text { Participants without } \\
\text { OSA }(\boldsymbol{N}=\mathbf{2 8})\end{array}$ \\
\hline & Median & IR & Median & IR & Median & IR \\
\hline Lab AI & 12.80 & 12.30 & 24.60 & 17.90 & 11.20 & 5.60 \\
Lab REM AI & 6.45 & 7.33 & 8.60 & 16.20 & 5.80 & 7.35 \\
Lab NREM AI & 17.9 & 17.38 & 21.40 & 15.40 & 16.00 & 17.60 \\
$1^{\text {st }}$ night home AI & 12.30 & 8.35 & 16.00 & 12.00 & 10.65 & 6.55 \\
$1^{\text {st }}$ night home REM AI & 4.95 & 5.98 & 5.05 & 9.75 & 4.74 & 7.03 \\
$1^{\text {st }}$ night home NREM AI & 15.70 & 15.13 & 21.65 & 21.08 & 14.70 & 10.30 \\
$2^{\text {nd }}$ night home AI & 9.7 & 6.4 & 13.00 & 8.90 & 8.60 & 5.95 \\
$2^{\text {nd }}$ night home REM AI & 9.05 & 7.23 & 10.80 & 13.95 & 7.50 & 7.70 \\
$2^{\text {nd }}$ night home NREM AI & 15.85 & 9.25 & 20.80 & 12.10 & 13.60 & 7.40 \\
\hline
\end{tabular}

Note: $\mathrm{AI}=$ arousal index; IR = interquartile range. Lab AI and $1^{\text {st }}$ night home AI (both in REM and NREM sleep) were normally distributed in patients with OSA but only medians are reported for easier comparisons. 
The comparison of the REM and NREM AI shows that participants had a significantly higher AI in their NREM sleep than in REM sleep in each recorded night with a large effect size (lab night $t(38)=3.43, p<.01, r=.48$; first night home $t(38)=4.04, p<.01, r=.55$ and second night home $t(38)=5.37, p<.01, r=.66)$. In terms of REM sleep duration, participants had less REM sleep in the laboratory than at home, $F(1.91,59.40)=5.03, p=.01, \eta^{2}=.14$ both during the first home night, $t(37)=3.32, p<0.01, r=.48$; and the second home night, $t(37)=$ 2.37, $p=0.02, r=.36$.

The level of consciousness in REM sleep was significantly closer to the level of consciousness in N2 sleep in laboratory night than during the home nights, which was caused by the difference between the lab and the second night at home, $t(17)=2.60, p=.02, r=.73$ while the differences were not significant between the lab and the first home night, $t(19)=1.63, p=$ $.12, r=.35$ or between the two nights at home $t(21)=0.38, p=.71, r=.08$.

Before conducting the regression analysis, the assumption of linearity between the predictor and the outcome variables was checked. There was a significant correlation between the relative level of consciousness (level of consciousness in REM sleep compared to level of consciousness in $\mathrm{N} 2$ sleep) and subjective wake time in the laboratory $(r=.40, p=.04)$ but not in the home nights $\left(r_{1 \text { st night home }}=-.01, p=.98 ; r_{2 n d \text { night home }}=-.34, p=.16\right)$. There were no significant correlations between the sleep diary and the PSG wake times (lab $r=.20, p=.06$; home $r=.39, p=.06$ ) - although the medium effect sizes suggest the study may have been underpowered to detect statistically significant results due to missing data in the home nights. Likewise, there were no significant linear relationships between the time participants spent in REM sleep and their perception of wake time in the three recorded nights, (lab $r=-.07, p=.50$; 
home $r=-.27, p=.14$ ). Due to the lack of collinearity between most of the proposed predictor and outcome variables, the planned regression analyses could not be executed.

\section{Discussion}

Consistent with the typical pattern of sleep state misperception among patients with chronic insomnia disorder, participants overestimated their wake time during the night compared to their objectively measured wake time. The discrepancy between the subjective and objective measures was similar to that which has been described among individuals with primary insomnia (Baglioni et al., 2013).

In contrast to the original study from which the REM instability hypothesis was generated, the AI was higher in NREM sleep than in REM sleep in the current sample (Feige et al., 2008). This may be due to the fact that we compared REM AI to the overall NREM AI, whereas in the other study the NREM arousal index was based solely on the AI in N2 sleep. In general, the micro-arousal frequency is highest in NREM N1 sleep and in REM sleep (Halasz, Terzano, Parrino, \& Bodizs, 2004). Thus, the high NREM AI in our sample may reflect an increased AI in N1. The micro-arousal distribution has not been described in other insomnia studies; therefore, we cannot make comparisons to previous literature other than to the REM instability hypothesis study cited above. For future comparisons, it will be important to determine the AI distribution in primary and comorbid insomnia samples.

With respect to the proposed predictors of subjective wake time, only the level of consciousness in the laboratory night correlated with sleep diary wake time, whereas PSG wake time, REM duration, and AI did not have a linear relationship with the outcome variable. There are several possible explanations for the discrepancy of results between the previous and the current study. 
Firstly, the sample characteristics were different: the previous study enrolled only individuals with primary insomnia, while most participants in the current sample had a comorbid sleep pathology or a sleep disorder other than insomnia disorder. Secondly, $26 \%$ of the sample in this study reported having chronic pain, which may have affected the amount and distribution of arousals. Furthermore, some of the participants were on psychotropic medications in the current study, which may have affected their sleep architecture, including the duration of their REM or the AI. Accordingly, it is possible that the REM instability hypothesis applies only to people who suffer from insomnia disorder but do not have any comorbid sleep, brain, or psychiatric pathology. Since the REM instability hypothesis was generated based on the results of one study, future studies should replicate the original results to establish if there is a consistent pattern of relationship between REM duration, $\mathrm{AI}$ and subjective wake time in primary insomnia. If such relationships are consistently present, it still remains to be explored if those findings are generalizable to clinical populations in which insomnia disorder is comorbid with other medical (including sleep and psychiatric) conditions. The results of this study suggest that the subjective wake time of a representative sample of patients with chronic insomnia following a mTBI may be influenced by variables other than REM duration and REM AI.

It was also proposed that an increased level of consciousness in REM sleep may bias patients' perception or recollection of REM sleep. There was a significant correlation between the relative level of consciousness in REM sleep and subjective wake time in one of the recorded nights, which suggests that it may be worthwhile to further explore the relationship between level of consciousness and sleep perception bias. We did not have a healthy, good sleeper control group in the present study but in future work, it will be important to compare the level of consciousness between healthy, good sleepers and individuals with insomnia disorder to establish if there is a 
significant group difference in the measure we used in the current study. It is also noteworthy that level of consciousness can be measured in several ways, including neuroimaging of DMN functional connectivity; neuroimaging of cerebral blood flow and glucose metabolism; and sleep EEG analysis. We used the latter method in the present study but future studies could incorporate multiple methods to investigate the relationship between sleep state misperception and the level of consciousness during sleep in chronic insomnia.

In addition to using multiple methods to measure the level of consciousness during sleep, the misperception of sleep as wakefulness can also be captured in various ways. The disadvantage of asking participants in the morning to estimate how much time they spent awake during the night is that the overestimation of wake time can be a result of either a misperception of sleep or can be caused by a retrospective memory bias. A more direct method to capture misperception is to wake participants from different sleep stages and ask them if they had just been awake or asleep. As an extension, they can also be asked what was in their minds just before they woke up as a means to collect information about their dream content. If the REM instability hypothesis is correct, a significantly larger percentage of insomnia patients would say that they were awake when woken from REM sleep and would be able to recall their dream content better than good sleepers. Furthermore, their dreams would reflect more "wake like" or realistic themes and scenarios than the dream content of good sleepers.

Finally, it is important to note arousals can be conceptualized more broadly than how micro-arousals are defined by current sleep scoring criteria (Halasz et al., 2004). High amplitude slow waves (for example delta bursts and $\mathrm{K}$ complexes) in isolation or in combination with low amplitude high frequencies, with or without autonomic arousal are integrated in cyclic alternating patterns (CAP) of brain activity (Parrino, Ferri, Bruni, \& Terzano, 2012). CAP 
reflects the brain's ability to protect sleep and at the same time maintain a responsiveness to internal or external environmental stimuli. CAP rate (a ratio of CAP time to NREM sleep time) is a measure of sleep instability in addition to the more widely used micro-arousal index. There is evidence to suggest that CAP rate is increased in primary insomnia; in particular during the period between PSG sleep onset and subjective sleep onset as well as in N1 and in N2 sleep (Parrino et al., 2012). It has been proposed that sleep instability - marked by high CAP rate in $\mathrm{N} 1$ and N2 sleep - may contribute to the sleep misperception of individuals with primary insomnia; specifically to the overestimation of sleep onset latency and to the misperception of fragmented sleep as continuous wakefulness (Parrino, Milioli, De Paolis, Grassi, \& Terzano, 2009). Interestingly, in OSA - the second most common sleep disorder in our sample - CAP rate is decreased (Parrino et al., 2012). It has not been described how comorbid OSA and insomnia changes the CAP profile of patients. Likewise, changes in CAP rate or profile have not been explored in TBI.

In summary, the findings of this study were consistent with previous research in primary insomnia with regards to the differences in subjective and objective wake time. In contrast, the NREM - REM distribution of AI was reversed in the current study compared to what previously has been described in good sleepers and among patients with primary insomnia. The results of the current study do not support the REM instability hypothesis of insomnia disorder among patients who suffer from chronic insomnia following a mTBI ; however, the study was limited by the presence of comorbid sleep disorders and psychotropic medication use in a sub-group of participants. Future studies should test the REM instability hypothesis among individuals with stand-alone and comorbid insomnia disorder. Furthermore, the potential role of level of consciousness in sleep perception may be a promising area to be further explored. 


\section{Chapter 7: Correlates of Sleep Quality and the Feeling of being Rested in the Morning among Patients with Chronic Insomnia Symptoms following Mild Traumatic Brain Injury}

People seek help for insomnia if they feel that there is "something wrong" with their sleep (or with their ability to obtain good sleep) based on their perception of having difficulty with sleeping or having poor sleep quality and not feeling refreshed from sleep. The subjective experience of poor sleep is a pivotal characteristic of insomnia disorder, yet, subjective sleep quality and the subjective perception of not feeling refreshed from sleep are not clearly conceptualized and understood (AASM, 2014). Given that our understanding is limited of these common subjective experiences of patients with insomnia disorder, an exploratory approach to investigate these constructs was undertaken.

Previous research on subjective sleep quality and the feeling of being restored from sleep is limited by: (1) predominantly using questionnaires in assessing sleep quality and feeling restored from sleep (2) primary focus on good sleepers and - to a lesser extent - on individuals with "primary insomnia" in studies investigating correlates of sleep quality and (3) an exclusive focus on patients with "primary insomnia" in studies attempting to determine the micro EEG correlates of subjective sleep perception (without involving specific measures of subjective sleep quality).

With regards to the limitation of assessment tools, a fundamental issue with the existing questionnaires is that their construct validity has not been adequately established. For example, scores of a widely used "sleep quality" questionnaire - the Pittsburgh Sleep Quality Index - do not significantly relate to prospective sleep quality ratings and are inflated by anxiety in psychiatric samples (Hartmann et al., 2015). Furthermore, there are only a few "non-restorative sleep" questionnaires and despite the promising initial psychometric results, consistent data of 
the construct validity of these questionnaires is not currently available (Drake et al., 2014; Vernon, Dugar, Revicki, Treglia, \& Buysse, 2010; Wilkinson \& Shapiro, 2013).

Another disadvantage of using retrospective questionnaires is that these tools may not capture the nightly variance of sleep quality and the daily changes in feeling refreshed from sleep. This could be especially problematic in assessing sleep in insomnia disorder, where the night-to-night variability of sleep is high but retrospective memory bias to poor sleep or sleepspecific anxiety may blur this daily variance and shift the overall rating in a negative direction (Hartmann et al., 2015).

Unidimensional scales are recommended to assess some subjective experiences, such as measuring pain intensity with VAS (Hjermstad et al., 2011). Also, ecological momentary assessment is recommended to capture the "in the moment" values and daily fluctuation of subjective experiences - for example fatigue, pain, or mood - in clinical populations to avoid memory biases that inflate retrospective subjective evaluations (Abdel-Kader et al., 2014; Curran, Beacham, \& Andrykowski, 2004; Hacker \& Ferrans, 2007; Kim, Kikuchi, \& Yamamoto, 2013; Kratz, Murphy, \& Braley, 2017; Powell, Liossi, Schlotz, \& Moss-Morris, 2017). Sleep quality and feeling (non)restored from sleep are subjective experiences with potential daily fluctuations but overall low values among patients with chronic insomnia. Accordingly, VAS appears to be a more useful tool for the prospective assessment of subjective sleep quality and feeling restored from sleep than retrospective questionnaires among patients with chronic insomnia disorder.

One key aspect of construct validity assessment is hypothesis testing with regards to the construct that is being assessed. One recent review commented that a particularly promising approach to understand subjective sleep perception in insomnia disorder would be to evaluate the 
intra-group relationship between objective EEG parameters and subjective sleep quality (Feige et al., 2013). Only a few studies have taken this approach hitherto (Feige et al., 2008; Maes et al., 2014; Normand, St-Hilaire, \& Bastien, 2016; Parrino et al., 2009; Perlis, Smith, Andrews, Orff, \& Giles, 2001). These studies involved only patients with primary insomnia and measured sleep misperception (i.e., the discrepancy between subjective and objective measures) but did not assess subjective sleep quality specifically. The findings of these studies suggest that REM sleep duration, CAP rate and high frequency EEG power density may be related to sleep misperception but these studies do not provide evidence of the EEG correlates of subjective sleep quality or the feeling of not being rested from sleep.

One can speculate that of the routine objective EEG measures, total sleep duration, time spent awake during the night, AI (as a measure of sleep fragmentation) and SWS may be related to subjective sleep quality and the feeling of not being restored from sleep. Intuitively, and according to previous research in good sleepers, if people are sleep deprived or struggle to fall/stay asleep at night, they may not feel refreshed in the morning and may evaluate their sleep to be poor (Akerstedt, Hume, Minors, \& Waterhouse, 1997). Deep sleep (SWS) is regarded to be the most restorative part of the sleep on the brain; thus, SWS deprivation may cause subjectively poor or non-restorative sleep (Akerstedt et al., 1997; Dijk, 2009; Shapiro, Bortz, Mitchell, Bartel, \& Jooste, 1981; Tononi \& Cirelli, 2014). Sleep fragmentation has been used as an indicator of poor objective sleep quality and it is related to daytime sleepiness among patients with sleep apnea (Parrino et al., 2012). Accordingly, sleep fragmentation may be associated with subjective poor sleep quality and not feeling refreshed in the morning. Likely no single feature is paramount but a constellation of variables may lead to a subjective experience of poor and non-restorative sleep. 
In addition to objectively measured EEG variables, subjective experiences that are common among patients with chronic insomnia disorder in general and in "post-concussion syndrome" in particular - i.e. depression, pain, or a subjective perception of curtailed and disrupted sleep - may also shape people's perception of their sleep quality or how refreshed they feel in the morning. When people have low motivation, feel depressed, or experience pain and fatigue, they may attribute these experiences to poor sleep or vice versa; they may assume that depression and pain cause low sleep quality and non-refreshing sleep (Harvey, Stinson, Whitaker, Moskovitz, \& Wirk, 2008). Likewise, subjective sleep quality evaluations and the extent to which someone feels rested in the morning may be based on the individuals' perception of how much they slept at night and how much time they spent awake during the night (Harvey et al, 2008).

This study extends previous research by exploring the association of daily, prospective VAS measures of sleep quality and feeling rested in the morning with routinely measured sleep EEG parameters, subjective sleep parameters, mood, anxiety, pain and fatigue among individuals who suffer from persistent insomnia symptoms following a mTBI.

\section{Objective and Hypothesis}

The last objective of the dissertation study (and the focus of the present chapter) was to explore the relationship among: (1) subjective sleep diary measures (i.e., SOL, WASO, total wake time, TST); (2) macro and micro EEG parameters (i.e., SOL, WASO, total wake time, TST, SWS duration, AI); (3) as well as subjective measures of pain, fatigue, anxiety, and mood, as predictors of perceived sleep quality and ratings of feeling rested in the morning. The hypothesis was that subjective total wake time would have the highest correlation with the outcome measures - this hypothesis is based on clinical experience/impressions when providing 
cognitive behavioural therapy for insomnia disorder (CBT-I) and on previous research on sleep quality in good sleepers (Akerstedt et al., 1997).

\section{Method}

Participants. Please see Table 3.2. and a descriptive summary of the sample in Chapter 3.

Procedures (relevant highlights). Participants kept the Consensus Sleep Diary for two weeks. They also rated their fatigue and pain (including headache) in the evenings before bedtime and they rated their sleep quality and the degree of feeling restored from sleep every morning using 100 mm VAS. They had three nights of PSG recording; the first of these nights was recorded in the sleep laboratory and two additional nights were recorded at home. A summary of these study procedures can be found in Figure 2.1. and in Table 2.1.

Apparatus and measures. The description of the relevant assessment tools and methods, including the VAS, the Consensus Sleep Diary, the DASS -21 as well as the laboratory and home PSG are described in Appendix D.

Analyses. Descriptive statistics summarized the distribution of variables. Repeated measures ANOVAs were used to compare the two-week average VAS ratings to the VAS ratings of the lab and home PSG nights to establish if the nights that included PSG recordings were representative with respect to sleep quality, feeling restored from sleep, pain and fatigue to the two-week study period. Repeated measures ANOVA compared if there was a significant difference between the VAS ratings across the three nights that included parallel PSG, Sleep Diary and VAS recordings. Finally, correlation analyses quantified the direction and strength of relationship between the variables of interest. 


\section{Results}

The VAS ratings were normally distributed. Descriptive statistics are reported in Table 7.1 .

Table 7.1.

Average Visual Analogue Scale (VAS) Ratings

\begin{tabular}{lcccc}
\hline & Mean $(S D)$ & $\begin{array}{c}\text { 95\% BCa CI of } \\
\text { the Mean }\end{array}$ & Skewness $(S E)$ & Kurtosis $(S E)$ \\
\hline Fatigue & $61.30(19.49)$ & $56.71-66.42$ & $-.45(.35)$ & $.06(.70)$ \\
Pain & $44.19(19.49)$ & $37.57-50.73$ & $-.18(.36)$ & $-.65(.70)$ \\
Sleep Quality & $36.10(11.07)$ & $32.27-40.32$ & $.13(.35)$ & $-.65(.70)$ \\
Feeling rested & $32.05(11.33)$ & $28.47-35.93$ & $.08(.35)$ & $-.51(.70)$ \\
\hline
\end{tabular}

Note: VAS were $100 \mathrm{~mm}$ lines. The score equals the distance from the left in millimeters. Higher ratings indicate higher fatigue, more pain, higher sleep quality and feeling more rested.

$\mathrm{SD}=$ standard deviation; $95 \% \mathrm{BCa} \mathrm{CI}=95 \%$ bias corrected accelerated confidence interval based on 1000 bootstrap samples, $\mathrm{SE}=$ standard error

The VAS ratings related to the three PSG recorded nights were similar to the two-week average VAS ratings for each variable $\left(\mathrm{F}_{\text {fatigue }}(3,84)=2.11, p=.11\right.$, partial $\eta^{2}=.02 ; \mathrm{F}_{\text {pain }}(3,81)$ $=0.99, p=.40$, partial $\eta^{2}=.04 ; \mathrm{F}_{\text {sleep quality }}(3,74.5)=1.02, p=.39$, partial $\eta^{2}=.04 ; \mathrm{F}_{\text {restorative sleep }}$ $(3,71.74)=1.02, p=.38$, partial $\left.\eta^{2}=.04\right)$; therefore, these VAS ratings adequately represent participant's fatigue, pain, sleep quality and sense of feeling restored from sleep during the twoweek study period.

Participants' perceived fatigue, pain, sleep quality and sense of being restored from sleep were also consistent across the three nights when PSG recordings were used $\left(\mathrm{F}_{\text {fatigue }}(2,62)=\right.$ $2.72, p=.07$, partial $\eta^{2}=.08 ; \mathrm{F}_{\text {pain }}(2,62)=1.45, p=.24, \eta^{2}=.05 ; \mathrm{F}_{\text {sleep quality }}(2,58)=0.77, p=$ $.47, \eta^{2}=.03 ; F_{\text {restorative sleep }}(2,58)=1.06, p=.35, \eta^{2}=.04$. Though the laboratory VAS ratings 
were similar to the home ratings, separate correlation analyses were conducted for the lab VAS ratings, because three variables included in the analyses (i.e., sleep diary SOL, SWS and AI) were significantly different in the laboratory recordings than in the home nights. Specifically, participants' perception was that they fell asleep faster in the sleep lab than at home. Additionally, they had less SWS and higher AI in the night they spent in the sleep laboratory than when they slept at home (for a more detailed description, please see the results section of Chapter 4). There were no statistically significant differences in the remaining independent variables across the three nights (for more detailed description of these variables across the three PSG night, please see the results section of Chapter 6). Since the ratings were similar in the two home PSG nights, the first and second night home ratings were averaged and these averages were used in the correlation analyses.

There was a strong and significant positive relationship between subjective sleep quality and feeling restored from sleep both in the laboratory $(r=.84$, BCa 95\% CI .62 - .93, $p<.01)$ and at home $(r=.80, \mathrm{BCa} 95 \% \mathrm{CI} .59-.92, p<.01)$, see Table 7.2. Additionally, there was a statistically significant relationship between anxiety and lower average ratings of "feeling rested" ratings during the study period, $r=-.35$, BCa 95\% CI.-.52 to - .15 $p<.01$. Neither sleep quality nor feeling rested had a statistically significant correlation with any of the subjective sleep diary measures, PSG measures, VAS measures of pain and fatigue, or with the DASS mood scores after Bonferroni corrections were applied. However, the point estimates of effect sizes show that subjective sleep quality had a strong correlation with sleep diary total time spent awake at night $(r=-.45)$ during the home nights (see Table 7.2). There was also medium correlation in the laboratory night between sleep quality and sleep diary WASO $(r=-.30)$. Feeling restored ratings 
had the strongest, meaningful correlation with total time spent awake at night $(r=-.46)$ at home. The absolute values of the remaining correlations were $r<.30$. 
Table 7.2.

VAS Sleep Quality and Feeling Rested Correlations

\section{CORRELATIONS WITH SUBJECTIVE SLEEP QUALITY}

\begin{tabular}{|c|c|c|c|c|c|c|}
\hline \multicolumn{4}{|c|}{ SLEEP LABORATORY } & \multicolumn{3}{|c|}{ HOME } \\
\hline Variables & $\begin{array}{l}\text { Correlation } \\
\text { coefficient }\end{array}$ & $\begin{array}{c}\mathrm{BCa} 95 \% \mathrm{CI} \text { of } \\
\text { the correlation } \\
\text { coefficient }\end{array}$ & $\mathrm{p}$ & $\begin{array}{l}\text { Correlation } \\
\text { coefficient }\end{array}$ & $\begin{array}{c}\text { BCa } 95 \% \text { CI of } \\
\text { the correlation } \\
\text { coefficient }\end{array}$ & $\mathrm{p}$ \\
\hline VAS rested & .84 & .73 to .91 & $<.01 *$ & .80 & .59 to .92 & $<.01 *$ \\
\hline PSG SWS & .27 & -.03 to .53 & .11 & -.18 & -.43 to .06 & .18 \\
\hline Diary WAKE & -.23 & -.44 to -.01 & .05 & -.45 & -.61 to .26 & .01 \\
\hline PSG SOL & -.13 & -.31 to .17 & .46 & .23 & -.07 to .49 & .11 \\
\hline PSG AI & .09 & -.30 to .14 & .46 & -.03 & -.35 to .28 & .82 \\
\hline Diary SOL & .03 & -.21 to .12 & .82 & -.25 & -.50 to .06 & .14 \\
\hline PSG TST & -.03 & -.27 to .20 & .79 & -.17 & -.47 to .21 & .40 \\
\hline PSG WASO & -.05 & -.26 to .17 & .65 & .03 & -.28 to .35 & .83 \\
\hline
\end{tabular}


Table 7.2. (cont.)

CORRELATIONS WITH FEELING RESTED/RESFSHED IN THE MORNING

\begin{tabular}{|c|c|c|c|c|c|c|}
\hline \multicolumn{4}{|c|}{ SLEEP LABORATORY } & \multicolumn{3}{|c|}{ HOME } \\
\hline & $\begin{array}{l}\text { Correlation } \\
\text { coefficient }\end{array}$ & $\begin{array}{c}\text { BCa } 95 \% \text { CI of } \\
\text { the correlation } \\
\text { coefficient }\end{array}$ & $\mathrm{p}$ & $\begin{array}{l}\text { Correlation } \\
\text { coefficient }\end{array}$ & $\begin{array}{c}\text { BCa } 95 \% \text { CI of } \\
\text { the correlation } \\
\text { coefficient }\end{array}$ & $\mathrm{p}$ \\
\hline Diary WASO & -.22 & -.45 to .00 & .06 & -.05 & -.35 to .25 & .66 \\
\hline Diary WAKE & -.20 & -.43 to .02 & .09 & -.46 & -.66 to -.20 & .01 \\
\hline Diary TST & .21 & -.02 to .40 & .08 & .22 & -.13 to .49 & .19 \\
\hline PSG WAKE & -.19 & -.40 to .07 & .09 & -.09 & -.36 to .53 & .68 \\
\hline PSG SWS & .17 & -.04 to .37 & .14 & -.17 & -.41 to .10 & .16 \\
\hline PSG TST & -.08 & -.33 to .15 & .47 & -.18 & -.52 to .21 & .39 \\
\hline PSG AI & -.03 & -.27 to .19 & .78 & -.02 & -.33 to .30 & .88 \\
\hline PSG WASO & -.03 & -.25 to .17 & .76 & .13 & -.15 to .40 & .36 \\
\hline Diary SOL & .05 & -.20 to .32 & .65 & -.26 & -.44 to -.07 & .03 \\
\hline PSG SOL & .00 & -.24 to .21 & .97 & $.36^{a}$ & .12 to .59 & .01 \\
\hline
\end{tabular}

Note: Correlations with magnitude .30 and above are bolded. $*$ denotes statistically significant results after Bonferroni corrections. VAS = visual analogue scale, WASO = wake after sleep onset, $\mathrm{SOL}=$ sleep onset latency, TST $=$ total sleep time; $\mathrm{SWS}=$ slow wave sleep, $\mathrm{AI}=$ arousal index, WAKE = total time spent awake at night. a: The direction of correlation between SOL and VAS rested is opposite to what would be expected, which cannot be easily explained. However, SOL is part of wake time, which has a medium, negative correlation with VAS rested ratings. BCa 95\% CI = bias corrected accelerated $95 \%$ confidence intervals 
Table 7.2. (cont.)

VAS Sleep Quality

\begin{tabular}{lccccc}
\hline & $\begin{array}{l}\text { Correlation } \\
\text { coefficient }\end{array}$ & $\begin{array}{c}\text { BCa } 95 \% \mathrm{CI} \\
\text { of the } \\
\text { correlation } \\
\text { coefficient }\end{array}$ & $\mathrm{p}$ & $\begin{array}{c}\text { Correlation } \\
\text { coefficient }\end{array}$ & $\begin{array}{c}\text { BCa 95\% CI of the } \\
\text { correlation } \\
\text { coefficient }\end{array}$ \\
\hline VAS fatigue & .08 & -.28 to .40 & .62 & -.08 & -.45 to .27 \\
VAS pain & -.05 & -.32 to .27 & .74 & -.10 & -.39 to .19 \\
DASS Anxiety & -.21 & -.40 to .00 & .05 & -.35 & -.52 to .15 \\
DASS Depression & -.16 & -.41 to .09 & .13 & -.17 & -.39 to .08 \\
\hline Note: Correlations with magnitude .30 and above are bolded. $*$ denotes statistically significant results after Bonferroni corrections
\end{tabular}

VAS = visual analogue scale, WASO = wake after sleep onset, $\mathrm{SOL}=$ sleep onset latency, TST = total sleep time; SWS = slow wave sleep, AI = arousal index, DASS Depression - Depression Anxiety Stress Scale 21 - Depression subscale

BCa $95 \% \mathrm{CI}=$ bias corrected accelerated $95 \%$ confidence intervals 
Table 7.3.

Correlations between the ESS, FSS, and VAS measures

\begin{tabular}{lcc}
\hline & $\begin{array}{c}\text { Correlation } \\
\text { coefficient }\end{array}$ & $\mathrm{p}$ \\
\hline ESS - VAS fatigue & .10 & .52 \\
ESS - VAS sleep quality & -.04 & .82 \\
ESS - VAS rested & -.03 & .86 \\
FSS - VAS fatigue & .07 & .50 \\
FSS - VAS sleep quality & .01 & .95 \\
FSS - VAS rested & -.03 & .78 \\
\hline
\end{tabular}

Note: The FSS and the VAS fatigue measure different constructs (i.e. fatigue-related functional impairment versus fatigue intensity). $\mathrm{BCa} 95 \% \mathrm{CI}=$ bias corrected accelerated $95 \%$ confidence intervals

\section{Discussion}

On average, participants reported that they were highly fatigued and were in pain when they went to bed in the evening. Their perceived sleep quality was low and they did not feel rested when they woke up in the morning. These subjective experiences are commensurate with persistent "post-concussion" symptoms and with the typical symptoms of chronic insomnia disorder. The fact that the scores on each VAS scale were normally distributed implies that the scale captured a range of experiences with regards to these variables and may reflect the distribution of scores in this clinical population.

The results of this study show that subjective sleep quality and feeling refreshed in the morning had the strongest correlations with subjective total time spent awake during the night and one of its components (i.e., WASO), while the correlations with subjective TST were somewhat weaker. Previous research on sleep quality in good sleepers showed that sleep quality 
was related to subjective ease of falling asleep, subjective "calmness of sleep", subjective number of awakenings, and the subjective perception of "sleeping through" (Akerstedt et al., 1997). This, in combination with the current findings suggests that being awake when one intends to sleep is a more important factor with regards to subjective sleep quality and "being refreshed" ratings than the perceived amount of sleep people get at night. CBT-I has mediumlarge effect sizes on subjective SOL and WASO but only small effect size on TST during treatment; nevertheless, patients report a substantive improvement in their sleep quality (GeigerBrown et al., 2015; Koffel, Koffel, \& Gehrman, 2015; Morin, Culbert, \& Schwartz, 1994). The results of this study provide a possible explanation for this phenomenon. These results also suggest that sleep treatments that reduce time spent awake during the night (e.g., CBT-I or hypnotic medications) may improve the subjective sleep quality and the feeling of being rested in patients who suffer from persistent insomnia following a mTBI.

The lack of relationship between subjective sleep perception and EEG macrostructure is not unexpected. The definition and diagnosis of insomnia disorder are based on subjective symptoms because there is no clear and consistent correspondence between conventionally scored (and often normal) EEG and patients' perception of poor sleep. Since changes in sleep macro-architecture are subtle and inconsistent among patients with insomnia disorder, the pursuit to find EEG correlates of subjective sleep perception has been focused on EEG micro-analysis over the past decade (Feige et al., 2013).

High sleep fragmentation has been regarded to be an objective measure of poor sleep quality and found to be increased in patients with chronic insomnia (Parrino et al., 2009). Nevertheless, in the present study there was no correlation between AI at night and subjective ratings of sleep quality or feeling refreshed in the following morning. There is evidence to 
suggest that CAP rate - another measure of sleep instability - correlates with daytime sleepiness in patients with OSA and it also has been suggested that it contributes to sleep misperception in insomnia disorder (Parrino et al., 2012). It is possible that CAP rate - rather than AI - is also related to subjective sleep quality and not feeling refreshed in the morning. Alternatively, sleepiness and sleep misperception may be separate constructs from subjective sleep quality and a feeling of being rested and these experiences may not correlate with known, objective measures of sleep instability. Indeed, neither sleepiness nor sleep misperception correlated with subjective sleep quality and feeling restored from sleep in our sample. This underscores the importance of understanding patients' conceptualization of sleep quality and what nonrestorative sleep means to them, and exploring the relationship of these experiences with other, apparently similar variables, such as sleepiness and sleep misperception. It is also noteworthy that there are numerous aspects of sleep and sleep-related physiological processes that were not measured in this study but may be associated with subjective sleep perception, for example EEG power, CAP rate and distribution, core body temperature, SWS-related growth hormone secretion, or brain activity associated with cortical hyperarousal.

It is important to emphasise that not feeling refreshed in the morning may be caused by factors other than sleep. For example, low mood, rumination, a sense of hopelessness or anxiety may contribute to the feeling of tiredness which people may attribute to low sleep quality or nonrestorative sleep (Harvey et al, 2008). In this study, there was a medium, statistically significant, negative relationship between the two-week average "feeing rested" and DASS - anxiety scores. This means that patients who were "on edge" and experienced physical symptoms of anxiety felt less rested in the morning. Previous research also showed that pre-sleep cognitive and somatic arousal as well as pre-sleep stress and worry were associated with perceived sleep quality 
(Akerstedt et al, 2012; Tang, Goodchild, Sanborn, Howard, \& Salkovskis, 2012). In contrast, the relationships between depression scores and the outcome measures were weak and nonsignificant. Of note, this study did not include daily VAS mood measures; instead it used the baseline DASS subscale scores in the analyses. In the current study - as in previous research there was a discrepancy between VAS and questionnaire results, showing that these tools measure different constructs and that the method of assessment (i.e. momentary versus retrospective; single item versus multiple items) has implications for the data collected (Table 7.3). Future research should include daily (instead of composite) mood ratings to determine if these ratings better correlate with daily measures of sleep quality.

Pain before bedtime/during the night may also disrupt sleep and impair sleep quality. Yet, the relationship between evening pain and morning VAS ratings were weak, despite the anticipated shared method variance. In accordance with the results of the current study, longitudinal studies have shown that sleep perception is not influenced by evening pain intensity in patients with insomnia disorder and chronic pain; rather, sleep duration and sleep fragmentation affects pain on the following day (Finan, Goodin, \& Smith, 2013; Tang, Goodchild, Sanborn, Howard, \& Salkovskis, 2012). Previous research in comorbid chronic pain and insomnia disorder suggests that the relationship between insomnia and pain is partially mediated by cognitive and behavioural variables that are targeted in CBT-I, which explains why CBT-I improves both sleep and pain, while CBT or medication treatment for pain does not cure chronic insomnia (Campbell et al., 2015; Lerman, Finan, Smith, \& Haythornthwaite, 2017; Martinez et al., 2014; Vitiello et al., 2014; Vitiello et al., 2013; Vitiello, Rybarczyk, Von Korff, \& Stepanski, 2009). 
When interpreting the results of this chapter, it is important to keep in mind that the discussed effect sizes were based on the point estimates and the confidence intervals show that there is a considerable variance in the strength and direction of relations between the studied variables in the population. Notwithstanding this limitation, the results of this study suggest that the experience of being awake when one intends to sleep and anxiety are the main determinants of the subjectively poor sleep among patients with persistent insomnia symptoms following mTBI.

The clinical implication of these results is that treatments that reduce anxiety and shorten wake time during the intended sleep period may improve subjective sleep quality and help patients with a history of mTBI and current insomnia disorder to feel more rested in the morning. However, these treatments (e.g. CBT) do not resolve OSA - which was comorbid with insomnia disorder in 17 individuals (34\%) of this sample. Even when there is a subjective improvement in sleep continuity and sleep quality during a course of an insomnia treatment, it is crucially important to identify and treat OSA to improve long-term health. It would also be interesting to know if an undetected/untreated OSA attenuates the effect of insomnia disorder treatments on subjective sleep quality and feeling rested after a sleep period. 


\section{Chapter 8: Summary and Conclusions}

This dissertation had two main goals: first, to determine the prevalence of sleep (including circadian) disorders that contribute to chronic insomnia symptoms following mTBI and secondly, to determine if objectively measured EEG and subjective variables are associated with the perception of poor sleep among patients presenting with chronic insomnia symptoms following a mTBI.

\section{Main Goal 1 - Diagnostic Findings}

The main finding with respect to the first goal was that 62 percent of patients with chronic insomnia symptoms had insomnia disorder, but only 28 percent had insomnia disorder as stand-alone condition (Figure 8.1 and 8.2 below). Most patients (72 percent of the sample) had a specific sleep disorder - other than an insomnia disorder (Figure 8.1 and 8.2); these conditions either contributed to the chronic insomnia symptoms or were fully responsible for the insomnia symptoms. These results have important implications for the sleep assessment and management of patients who report persistent insomnia symptoms following a mTBI.

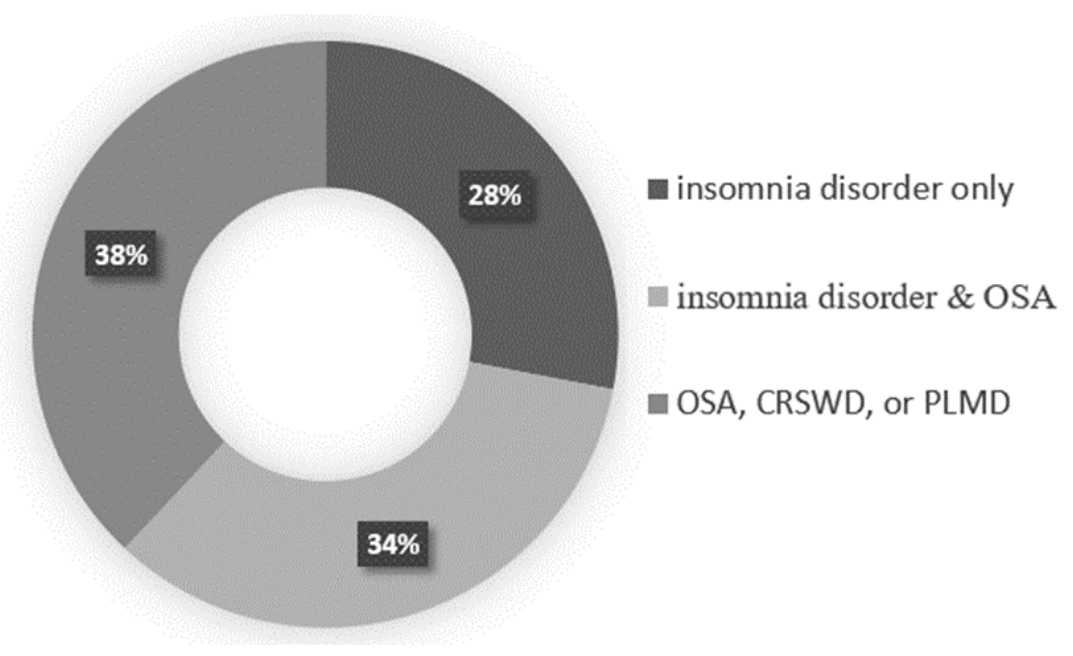

Figure 8.1. Summary of the Sleep and Circadian Diagnostic Findings 


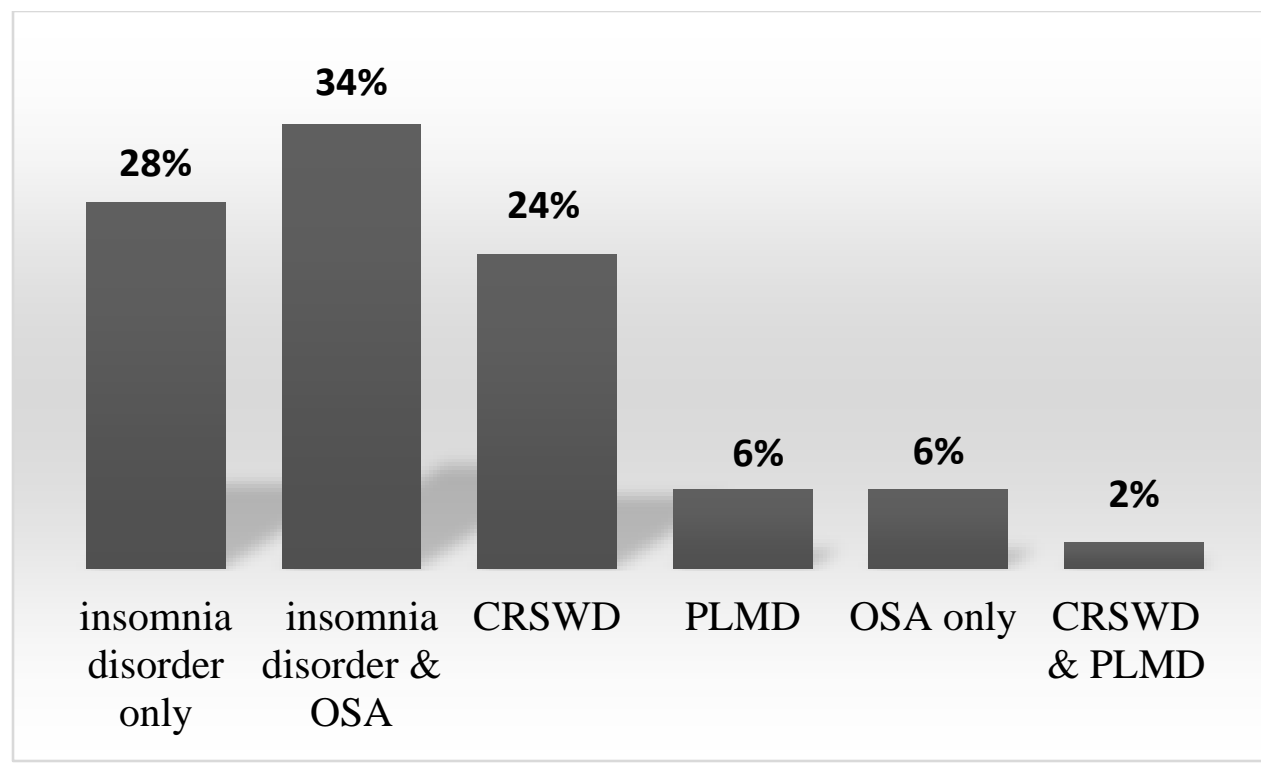

Figure 8.2. Sleep and Circadian Diagnostic Findings - Break-down of specific disorders

Implications for Assessment. The most important implication with regards to assessment is that a multi-method assessment is required for patients who report persistent, clinically significant insomnia symptoms following a mTBI. It is important to emphasize that although the general, North American insomnia assessment guidelines do not require the use of objective assessment tools as part of a standard insomnia assessment, this thesis makes it apparent that selected objective sleep assessment methods are imperative in the clinical evaluation of patients with chronic insomnia following a mTBI.

More than $80 \%$ of patients above the age of 50 years and approximately half of the group above 40 had OSA in this clinical sample, which suggests that it would be desirable to offer PSG assessment for individuals above 40 who sustain significant, chronic insomnia symptoms (e.g. ISI > 11) following a mTBI (Figure 8.2). Using the OSA screening STOP-BANG Questionnaire may also help to decide which patients would most likely benefit from a PSG assessment: in the present study a cut-off score of three on the STOP-BANG Questionnaire had high specificity 
(89\%) compared to the PSG and at this cut-off score $80 \%$ of the patents were correctly identified as having OSA versus not having OSA.

It is possible that many individuals who were diagnosed with OSA had this sleep disorder before the TBI but the OSA symptoms may have become apparent or may have been amplified and therefore possibly misdiagnosed as "post-concussion symptoms" - following the injury. In other cases, OSA may have developed following the injury, for example, if the enervation of the pharynx had been injured during a whiplash injury (Guilleminault et al., 2000) or if the individual gained weight due to the lifestyle changes in the post-injury period or due to interactions with a comorbid insomnia disorder (for the description of the possible relationship between OSA and insomnia following a mTBI, please see Chapter 4). Ideally, all individuals above 40 and those who obtain scores $\geq 3$ on STOP-BANG will be offered a PSG as early as three months post injury to ensure early diagnosis and treatment. If the first PSG does not show OSA in a patient of this age group, but the patient suffers from persistent post-concussion insomnia symptoms and/or gains a significant amount of weight following the injury, it may be worthwhile to repeat the PSG in a year.

Laboratory sleep studies are relatively easily available in certain geographical locations (for example in the Greater Toronto Area) but are more difficult to access in other regions. Home PSG is a cheaper alternative to laboratory-based PSG and has been approved for OSA screening (Schutte-Rodin et al., 2008). We used a home PSG system in the present study that provides a full PSG recording (including a full EEG and leg movements). It has several advantages over simpler portable OSA monitors and could be used not just for screening but also for diagnostic purposes. With regards to OSA diagnosis, the home recordings detected mild OSA and increased AHI in REM sleep when these events were not apparent in some of the laboratory recordings. 
This suggest that home recording may identify patients who are more likely to develop clinically significant OSA in the future and may require repeated testing if the sleep/post-concussion symptoms do not resolve. It is noteworthy that $95 \%$ of the present sample was able to provide home PSG recordings without professional assistance and rated the home PSG device easy/somewhat easy to use, which implies that (subjective) cognitive problems following a mTBI do not preclude the possibility of conducting home PSG studies.

In addition to detecting OSA, PSG was also useful to identify sleep abnormalities - for example mild OSA, high apnea index in REM sleep, or high PLM index - in cases when the patients reported chronic insomnia symptoms on the ISI and during the clinical interview, but their sleep dairy data were normal with respect to subjective wake time and sleep efficiency. In these cases, the PSG findings were important to verify that there is a sleep abnormality and these PSG results informed treatment.

The second most common sleep disorder (other than insomnia disorder) after OSA was DSWPD. The prevalence of this condition was extraordinarily high, in particular among patients who were in their late teens and twenties. Accordingly, clinicians have to be vigilant to recognize the symptoms of DSWPD when patients in this age group report symptoms of chronic insomnia (Table 5.2.). Actigraphy and DLMO testing play an important role in the assessment of this condition.

It is important to remember that evidence-based assessment must precede the treatment of sleep disorders, since the assessment provides information for diagnosis and treatment planning. Unfortunately, the current Ontario guidelines for management of persistent post-concussion sleep-wake disorders is not based on this fundamental principle (ONF, 2013). 
The current "Ontario Neurotrauma Foundation Guidelines for Concussion/Mild Traumatic Brain Injury and Persistent Symptoms" recommends that every patient with persistent sleep-wake disturbances following a mTBI be placed on a sleep hygiene program (ONF, 2013). It further recommends that they complete a cognitive behavioural therapy for insomnia program or to take medications, such as trazodone, mirtazapine, tricyclic antidepressants or quetiapine supplemented with magnesium, zinc or melatonin. If the sleep-wake disturbances persist, patients can be offered further treatments and lifestyle changes, such as acupuncture, mindfulness based stress reduction, or exercise. At this point, they should be screened by their health care providers for sleep-related breathing disorders, nocturnal seizures, periodic limb movements or narcolepsy. If there is a suspicion that any of these conditions are present, the patients should be referred for a sleep specialist consultation. To summarize, the Guideline recommends, in order: (1) symptom assessment (i.e. insomnia or excessive daytime sleepiness); (2) symptom treatment (irrespective of what the symptoms are and which sleep disorders cause these symptoms); (3) screening/assessment by non-sleep specialist for disorders, such as PLMD which cannot be assessed without PSG and - as the last step - (4) referral to a sleep specialist.

One fundamental issue with the Guideline is that it recommends treatment of symptoms before patients are given the opportunity to participate in a formal sleep assessment. If professionals follow these recommendations, evidence-based sleep assessment may be delayed by months or years, while patients may be placed on ineffective (e.g. sleep hygiene ${ }^{16}$ ) or potentially harmful (e.g. tricyclic antidepressant or antipsychotic) interventions, while their sleep disorders remain undiagnosed and untreated. It is important to reiterate that ongoing, clinically significant insomnia symptoms necessitate a sleep and circadian assessment. The results of this

\footnotetext{
${ }^{16}$ Sleep hygiene is a necessary but not sufficient component of insomnia disorder treatment. Since it is ineffective as a stand-alone intervention, it is used as placebo in behavioural insomnia treatment trials.
} 
study suggest that subjective assessment methods are necessary but - in most cases - not sufficient to arrive at an accurate diagnosis that informs treatment planning in this clinical group.

Implications for Treatment. Once the diagnosis is made, the treatment should be based on the best evidence available for the sleep disorders that we aim to treat. With regards to insomnia disorder, the first line treatment is cognitive behavioural therapy for insomnia (CBT-I). However, only one study has tested CBT-I among patients with TBI. This study included 11 adults (only one had mTBI and two had mild-moderate TBI, while most participants had more severe brain injury) and used a single-case design to test the potential efficacy of CBT-I in this clinical group. The results were promising but the efficacy of CBT-I in treating persistent postconcussion insomnia has not been tested in a clinical trial (Ouellet \& Morin, 2007).

The results of this study suggest that CBT-I may be used as a stand-alone treatment option only in approximately one-quarter of patients who suffer from chronic insomnia symptoms following a mTBI. These are the patients who were diagnosed with an insomnia disorder and did not have comorbid sleep disorders. The dissertation provides evidence to suggest that there are specific CBT-I treatment targets in this group of patients. Specifically, participants had high scores on questionnaires that assess maladaptive psychological factors that perpetuate insomnia disorder, for example sleep effort, dysfunctional beliefs and attitudes about sleep, sleep-specific anxiety and rumination about the daytime symptoms of insomnia. Another, relevant finding of this study is that there was an unusually high prevalence of excessive daytime sleepiness $(\mathrm{ESS} \geq 11)$ in this sample compared to general insomnia disorder samples. CBT-I temporarily increases daytime sleepiness; thus, this treatment may increase the risk for repeated accidents or injuries when used to treat insomnia disorder among individuals with a recent mTBI. Accordingly, it would be worthwhile to test a modified CBT-I protocol that does not 
involve an acute sleep restriction, allows short naps, and includes instructions for extra caution when people drive during treatment.

In addition to being a stand-alone treatment option for patients with insomnia disorder, CBT-I might also be a potential treatment option for patients who suffer from comorbid OSA and insomnia disorder (34\% of the current sample). However, there is no sufficient data even in the general insomnia literature as to what is the best way to implement CBT-I in patients with comorbid insomnia disorder and OSA (e.g. combined or consecutive treatment) to improve adherence to OSA treatment. According to the above, CBT-I could be a treatment option for approximately two-thirds of patients with chronic insomnia disorder that develops/worsens following a mTBI; either implemented as a stand-alone treatment or as an intervention that is combined with OSA treatment. As an important future research agenda, clinical trials are required to test the efficacy of CBT-I among patients with insomnia disorder who had a relatively recent mTBI.

Another important clinical implication of the dissertation results is the potential role of melatonin in post-concussion sleep treatment. Melatonin is one of the recommended treatments of certain CRSWDs, including DSWPD, which was the most common circadian diagnosis in this sample. One study has tested the efficacy of melatonin for the treatment of DSWPD following whiplash injury (van Wieringen, Jansen, Smits, Nagtegaal, \& Coenen, 2001). This study showed that $5 \mathrm{mg}$ melatonin administered five hours prior to the DLMO significantly advanced DLMO and wake time. This result suggests that melatonin - taken in the appropriate time in relation to the DLMO - could be used in the treatment of DSWPD following mTBI. It would be important to conduct melatonin testing when the patient appears to have circadian sleep-wake disorderfor a more detailed description, please see Chapter 5. Unfortunately, DLMO testing is not easily 
available. The results of this dissertation suggest that DLMO could be estimated with acceptable precision based on sleep diary wake time (wake time plus 13 hours). When using this calculation, it is important to collect sleep data when patients are on a free sleep schedule.

\section{Main Goal 2 - Findings Related to Sleep Perception}

The second goal of the dissertation was to determine which objective sleep parameters and subjective variables were associated with subjective wake time, subjective sleep quality and the degree to which participants felt restored/refreshed in the morning.

The first subjective outcome measure was subjective wake time. With regards to this outcome measure, this study detected the expected subjective overestimation of wake time. Unlike in the original study proposing the REM instability hypothesis (Feige et al., 2008), however, PSG wake time and REM sleep duration did not correlate with subjective estimates of wake time in the current mTBI sample. One explanation for this discrepancy could be that the REM instability hypothesis is valid only in primary insomnia. In contrast, one could make the point that REM sleep is also compromised in various sleep disorders, including OSA, which was a prevalent sleep disorder in the current sample. Some argue that insomnia and OSA are "two sides of the same coin", evidenced by the high rate of co-occurrence, the shared clinical symptoms, and the linked pathophysiology. The present study also suggests that OSA and insomnia may have shared psychopathology. This is indicated by the same degree of endorsement of dysfunctional sleep-specific cognitions and sleep-specific worry in every diagnostic group in this study, irrespective of the final clinical diagnoses. If there are shared pathophysiological and psychopathological mechanisms between OSA and chronic insomnia, the factors that shape subjective sleep perception in these conditions may also overlap. In this context, the lack of replication of the REM instability hypothesis is less easily explained. 
This study extended the REM instability hypothesis and proposed that the level of consciousness in REM sleep would predict subjective wake time. This was only partially supported by the results: there was a medium-strong correlation in the night participants spent in the laboratory, when their level of consciousness in REM sleep was closer to the level of consciousness in N2 sleep than during the home nights. As it was discussed in Chapter 6, it would be worthwhile to further compare the level of consciousness in REM sleep in good sleepers, patients with insomnia and patients with TBI and explore its relationship with sleep perception bias. Given the limited supporting data, there is also a need for further research to replicate the original REM instability hypothesis results in primary insomnia samples.

The second sleep perception outcome measure was subjective sleep quality. The results showed strong, negative relations for sleep quality with anxiety and sleep diary wake indices. These results suggest that anxiety (specifically psycho-physiological arousal) and subjective wake time should be targeted in treatments that aim to improve the subjective sleep quality of patients who suffer from post mTBI chronic insomnia symptoms.

Finally, this study assessed how rested/refreshed participants felt after they woke from sleep in the morning. In the sleep literature, "non-restorative sleep" has been defined as a "subjective feeling of being unrefreshed upon awakening that may be the result of poor sleep quality or unrestful sleep" (Drake et al., 2014) or "a subjective experience that sleep has not been sufficiently refreshing or restorative" (Wilkinson \& Shapiro, 2013). According to these definitions, at least three conditions should be met: (1) ratings of subjectively poor sleep quality; (2) feeling unrefreshed after a sleep period; (3) being unrefreshed is caused by or is attributed to poor sleep. The current study showed a correlation between sleep quality and feeling rested ratings but not to the extent that would suggest that these are the same constructs. Previous 
research also found that sleep quality and being refreshed from sleep are separate constructs (Akerstedt, 1999).

In the current study EEG measures were not related to the feeling of being rested in the morning but the results showed a strong correlation with subjective variables, including sleep quality, subjective wake time and anxiety. A limitation of this study is that - similarly to previous research - attributions were not assessed. It is recognized in health psychology that the same physical experiences may have various subjective interpretations and attributions and these attributions influence illness behaviour and experience (Cameron \& Moss-Morris, 2004). Future research should explore what individuals mean by "non-restorative sleep", how they distinguish between poor sleep quality and not feeling rested after a sleep period, and what attributions good sleepers, patients with sleep disorders, and patients with persistent TBI symptoms make when they do not feel rested in the morning.

\section{Additional Results}

Questionnaire scores pertaining to post- concussion symptoms (RPQ), subjective sleepiness (ESS) and psychological, sleep-specific variables (DBAS, GSES, APSQ and DISRS) also have yielded interesting results with potential clinical implications.

With respect to "post-concussion symptoms", the worsening of sleep and fatigue items had the highest scores on the RPQ. Although this is not surprising, it is a noteworthy finding, because it indicates that patients experiencing clinically significant insomnia symptoms following a mTBI could be identified based on these two items on the RPQ. Since the RPQ (or similar, post-concussion symptom questionnaires) are already in the assessment "toolkit" of sport medicine and head injury clinics, these clinics could use these tools to identify patients who would benefit from additional, sleep-specific assessments. 
Another, important finding was that the cognitive items - including forgetfulness, poor concentration, and difficulty with thinking- had the second highest scores on the RPQ. These subjective cognitive problems are easily attributed to the head injury and perhaps it is less appreciated these cognitive difficulties are also common daytime consequences of various sleep disorders, as illustrated in Figure 8.3 below. If symptoms that patients experience post-TBI are fully attributed to the TBI, it may hinder the diagnosis of potentially well-treatable conditions, including sleep disorders. Therefore, it is important to educate patients and health care professionals of the overlapping symptoms of sleep disorders and mTBI in order to facilitate accurate diagnosis and effective treatment. To this end, I have jointly co-authored an educational booklet on concussion that includes a chapter on sleep problems, sleepiness and fatigue (Zalai, Margaliot, Shapiro, \& Cusimano, 2018).

\section{SHARED SYMPTOMS OF INSOMNIA AND CONCUSSION}

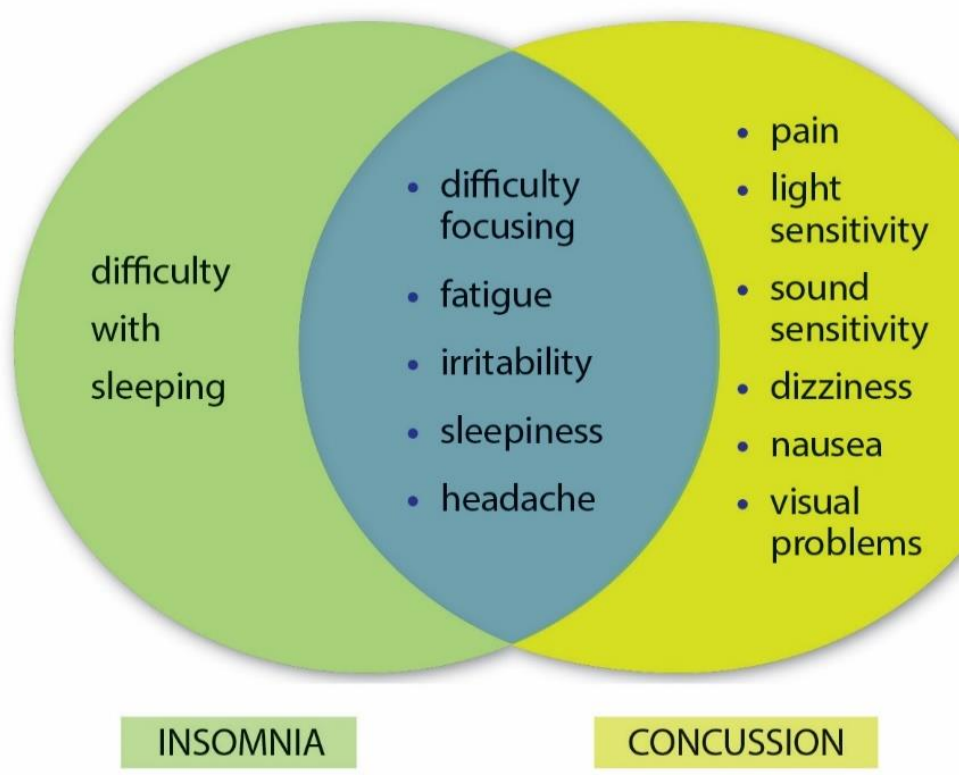

Figure 8.3. Overlapping Symptoms of Insomnia and mTBI (page 43 in Zalai at al., 2018) 
A further interesting finding was the high frequency of excessive daytime sleepiness in each sleep disorder diagnostic category in this study. This is an unusual result because excessive daytime sleepiness is not characteristic of insomnia disorder although it is relatively common in OSA and in other sleep disorders. The high rate of excessive daytime sleepiness among patients whose only diagnosis was insomnia disorder cannot be explained by age or short sleep duration, because age was not related to being sleepy or not sleepy in this study and most participants with insomnia disorder had normal sleep duration. It can be inferred from the above that the unusually high rate of subjective excessive daytime sleepiness among participants with insomnia disorder (without OSA) was due to other factors, most likely due to the TBI. Indeed, sleepiness is a common symptom after a mTBI. It also has been shown that there is a general slowing and desynchrony of wake EEG following a mTBI, which is an electrophysiological sign of high sleep pressure and local sleep (Modarres, Kuzma, Kretzmer, Pack, \& Lim, 2016). It appears that patients who have persistent insomnia following a mTBI form a unique insomnia group who experience both nighttime sleep difficulties and excessive subjective daytime sleep propensity. This has relevance for treatment, as was previously described in this chapter.

In addition to the concussion and sleepiness scales, the psychological sleep-specific questionnaires (DBAS, GSES, APSQ and DISRS) also yielded interesting information in this study. Firstly, the mean scores on these questionnaires were at least as high as the mean scores are in non-TBI insomnia samples suggesting that non-adaptive sleep-specific psychological factors are present when individuals experience insomnia symptoms following a mTBI. According to Spielman's stress-diathesis model, psychological factors take on a psychopathological role in perpetuating insomnia symptoms after the direct impact of an original trigger (in this case the impact of the TBI) subsides (Spielman et al., 1987). As a result, 
insomnia decouples from the trigger and becomes an independent condition (i.e. insomnia disorder) that can effectively be treated if the maintaining factors are targeted, as it is done in CBT-I.

The sleep-specific psychological variables assessed in this study are conceptualized to be specific to insomnia disorder. Curiously though, participants in each sleep diagnostic category

obtained scores in the clinical range on these questionnaires. In other words, patients whose final diagnosis was not insomnia disorder endorsed dysfunctional beliefs and attitudes about sleep, anxiety and preoccupation about sleep, sleep effort, and rumination about daytime symptoms of insomnia to the same extent as patients with insomnia disorder did. Only one previous study compared dysfunctional beliefs and attitudes about sleep in different sleep disorders (Cronlein et al., 2014). Consistent with the results of the current study, there was no difference in the DBAS scores across sleep disorder diagnostic categories. The current study extends those previous results, showing that not only dysfunctional beliefs and attitudes about sleep but also preoccupation and worry about sleep, rumination about daytime symptoms of insomnia and sleep-effort are similar across sleep disorder diagnostic groups.

In the current study the psychological sleep-specific questionnaire scores significantly correlated with the ISI. Although correlation does not mean causality, a parsimonious explanation is that when individuals perceive or think that they have a sleep difficulty, this may activate dysfunctional cognitions about sleep and lead to sleep-focused worry, rumination, anxiety, and sleep effort independent of the clinical sleep disorder diagnosis. The clinical importance is that these psychological factors may cause psycho-physiological arousal, which, in turn may amplify the sleep problem and maintain (subclinical) insomnia. It remains to be 
established if these psychological factors should be targeted in treatment of sleep disorders other than insomnia disorder.

\section{Study Limitations}

In the evaluation of the results of this study, a number of factors merit consideration. Firstly, these results apply to patients with chronic insomnia symptoms who had a mTBI between three months and 24 months before the sleep assessment. The percentage of various sleep disorders may be different among individuals who do not experience insomnia symptoms following the TBI or who had the TBI more than two years before a sleep assessment.

Secondly, participants of this study agreed to have a sleep assessment at a sleep clinic, which suggests that they may represent severe cases of insomnia or the ones who are most distressed about their sleep. At the same time, these are the individuals who are most likely referred for a sleep assessment following a mTBI. With regards to the inclusion criteria, the duration of insomnia symptoms and the sleep history were based on subjective report. It has been noted that individuals may not report symptoms that had been present before the injury; thus, it is possible that some participants had ongoing insomnia symptoms (or symptoms of other sleep disorders) when they suffered the mTBI. This did not change the current sleep disorder diagnoses but precludes making inferences about a potential causal role of the mTBI.

Patients who had comorbid medical conditions and who took medications were included in this study to obtain a representative sample of patients seen at medical settings following a mTBI. This was an advantage in the clinical diagnostic part of the study (Objectives 1 and 2) but was a limitation in the more theoretical parts (Objectives 3 and 4) that ideally would have included participants without comorbidities. 
With regards to assessment tools, the sleep disorder diagnoses were based on wellestablished assessments tools and methods; however, the measurement properties of these tools had not been examined in patients with a history of post-mTBI chronic insomnia. On the positive side, this study provides preliminary information for the psychometric properties of the assessment tools in this clinical group.

Finally, this study did not include comparison/control samples which would have strengthened the study and would have been particularly important for Objective 3 (testing the REM instability hypothesis).

\section{Future Directions}

The dissertation has answered the primary research questions and at the same time generated questions for future research. Ideas for future research studies involving individuals with mTBI are listed below.

- Examine the psychometric properties of insomnia and sleep apnea screening tools among patients with mTBI.

- Examine the relationship between subjective excessive daytime sleepiness (e.g. ESS scores) and the standard measure of objective excessive daytime sleepiness (Multiple Sleep Latency Test) in patients with/without insomnia disorder following a mTBI.

- Compare laboratory and home PSG in a cross-over design study over multiple nights in patients with insomnia following mTBI.

- Test the feasibility and clinical utility of sleep assessment triaging models among patients with post-concussion syndrome.

- Test the potential efficacy of CBT-I among patients with insomnia disorder following a mTBI. 
- Test the efficacy of melatonin/bright light as treatment interventions for patients with CRSWD following a mTBI.

- Explore the concept of subjective sleep quality among good sleepers, individuals with insomnia disorder, and individuals with persistent post-concussion symptoms.

- Explore attributions of poor sleep quality and not feeling rested in healthy individuals, patients with insomnia, and individuals following a mTBI.

- Examine symptom attributions among patients with mTBI and their health care providers. Determine how these attributions are related to health care utilization and mTBI outcomes.

- Compare level of consciousness in REM sleep between healthy good sleepers, patients with insomnia disorder, and patients with mTBI without sleep disorders.

- Determine arousal distribution and CAP rate as well as the relationship of these sleep instability measures to subjective sleep quality in primary insomnia disorder, in insomnia disorder following mTBI and in patients with mTBI without sleep disorders.

\section{Conclusions}

This dissertation reveals that only $28 \%$ of patients who are referred for an assessment of chronic insomnia within the first two years following a mTBI have insomnia disorder as a standalone condition. In reality, most patients have additional/other sleep disorders that require a multi-method diagnostic sleep and/or circadian assessment. The study identified that the most common non-insomnia sleep disorders among patients referred for an assessment of post-mTBI chronic insomnia are OSA and CSWRD and this finding can be used to guide patient triaging and inform diagnostic sleep assessments in this clinical group. Accurate diagnostic assessment is 
the prerequisite of effective treatment. Given that patients with chronic insomnia symptoms have adverse long-term mTBI outcomes and that sleep disorders can contribute to persistent "postconcussion symptoms", accurate diagnosis and effective treatment of sleep disorders may hasten patients' recovery and improve long-term mTBI outcomes. 


\section{Appendix A: Explanation of Insomnia Disorder Definitions}

Chronic insomnia disorder (also referred to as chronic insomnia) is characterized by persistent difficulties with initiating or maintaining sleep and/or waking up earlier than one desires (Diagnostic and Statistical Manual of Mental Disorders, (DSM-5 ), 2013; International Classification of Sleep Disorders: Diagnostic and Coding Manual, 2014). Typically, SOL of more than half an hour and/or WASO of more than half an hour are considered clinically significant sleep initiation or maintenance problems. These night-time symptoms are associated with perceived daytime consequences of poor sleep including fatigue, reduced motivation or desire to take initiative, feeling sleepy, subjective cognitive impairment, mood disturbance, proneness to errors or accidents attributed to poor sleep, concerns about or dissatisfaction with sleep, and impaired performance in important (e.g. social, vocational, academic) areas of functioning.

Insomnia is defined as a clinically significant disorder only in the presence of at least one of the above daytime complaints. For frequency and duration criteria to be met, the sleep difficulty and daytime symptoms must occur at least three times per week for at least three months, despite adequate sleep opportunity and circumstances for sleep. Some patients have sleep difficulties that last for weeks and are interspersed with periods of good sleep. Chronic insomnia disorder can be diagnosed if the sleep difficulty is recurrent, even if the periods of poor sleep do not last for three consecutive months. The diagnosis of short-term insomnia disorder should be assigned in cases where frequency and duration requirements for chronic insomnia are not met (International Classification of Sleep Disorders: Diagnostic and Coding Manual, 2014).

From a clinical perspective, it is important to distinguish between insomnia symptoms and insomnia disorder. Insomnia symptoms (i.e. difficulty falling asleep or staying asleep or waking up too early) can arise due to a variety of sleep disorders including OSA, restless legs 
syndrome, PLMD, or CRSWD. A diagnosis of insomnia disorder is assigned only if the insomnia symptoms are independent in their onset, if they vary over time from the symptoms of the comorbid sleep disorder, or if symptoms persist despite adequate treatment of the comorbid sleep disorder. The identification of psychopathological insomnia-maintaining factors aids differential diagnosis. For example, OSA can cause frequent awakenings during the night and a final awakening earlier than desired in the morning. These insomnia symptoms can be fully accounted for by sleep-disordered breathing and may completely resolve when the OSA is treated. In this situation, an insomnia disorder diagnosis is not made. Conversely, if an individual has symptoms of insomnia disorder before symptoms of OSA first appear, has substantial difficulties resuming sleep after nighttime awakenings because of intense worry about sleep, or has sleep difficulties that persist after the OSA is resolved, a comorbid chronic insomnia diagnosis is appropriate.

Individuals may also report insomnia symptoms when they do not have any underlying sleep disorders. For example, they may have sleep initiation or maintenance difficulties or may habitually wake up early in the morning without being concerned about their sleep or experiencing daytime symptoms. In this case, although insomnia symptoms are present, an insomnia diagnosis is not made. It may also be the case that an individual is concerned about sleep initiation or maintenance difficulties or early-morning rising even though there is no evidence (via sleep diary or PSG) of any sleep problem. This can occur when individuals have unrealistic expectations about sleep; for example, if one believes that he or she should sleep until at least 7:00 am every morning or should sleep through the night without any awakening. It is also not unusual for an individual's subjective perception of sleep to be different from objectively measured sleep parameters. This occurs, for example, when patients overestimate the 
time it takes to fall asleep or the time they spend awake during the night or underestimate their sleep duration. In these cases, insomnia disorder can be diagnosed on the basis of subjective complaints. However, in insomnia research, and—based on the clinician's judgement—in clinical practice, the diagnosis may be deferred in the absence of a detectable sleep abnormality. 


\section{Appendix B: Insomnia Assessment}

Ideally, insomnia evaluation incorporates a multi-method assessment, summarized in Table 1.1. In clinical practice, especially in non-specialized settings, it is not uncommon for clinicians to conduct only a symptom evaluation. Although this approach can confirm the presence of self-reported insomnia symptoms, it does not provide data that would corroborate patients' retrospective, global self-reports or allow appropriate differential diagnosis and individually-tailored, evidence-based treatment planning. At a minimum, assessment should include multiple, complementary subjective assessment tools; for example, questionnaires, a standard sleep diary, and a clinical interview. A sleep history, including a semi-structured sleep interview and questionnaires (in particular the Insomnia Severity Index or the Pittsburgh Sleep Quality Index), is an essential component of insomnia research (Buysse et al., 2006).

Additionally, taking a medical history and conducting a semi-structured psychiatric interview, preferably using the Structured Clinical Interview for DSM (SCID), Axis I, is recommended in research settings.

Self-report scales yield information about insomnia severity, SOL, TST, WASO, or endorsement of psychological factors associated with insomnia, including dysfunctional beliefs and attitudes, excessive worry and rumination about sleep, and subjective psychophysiological arousal. Most symptom questionnaires have demonstrated differences between patients with insomnia and good-sleeper samples. Some - for example, the ISI and the DBAS—-have also demonstrated sensitivity to treatment-related change. Thus, these questionnaires can be used as outcome measures and can be given to patients repeatedly to track changes over the course of treatment. One disadvantage of self-report scales is that they provide retrospective global assessments that are influenced by patients' memories, sleep (mis)perceptions, and moods, among other factors. Only a few measures have been validated in specific populations. Since 
most cognitive measures have not been used in specific populations-for example, in older adults or in populations with medical disorders - the contribution of specific cognitive factors to insomnia is unknown in these populations. Furthermore, most cognitive measures do not have clinical cut-offs to distinguish individuals with insomnia from good sleepers and from patients with other sleep disorders.

Sleep diaries are prospective assessment tools completed in the morning that address the previous night's sleep and the previous day's behaviours. A standard “consensus sleep diary” contains daily entries about bedtime, time of first sleep attempt, SOL, number of awakenings during the night, WASO, terminal WASO (time spent in bed between the final awakening and rising time), rising time, ratings of sleep quality and feelings of refreshment from sleep, as well as daytime naps, hypnotic use, and alcohol and caffeine consumption (Carney et al., 2012). TIB, wake time (SOL + WASO + terminal WASO), TST and SE are calculated from sleep diary data and used in treatment planning and as outcome measures in cognitive behavioural therapy for insomnia. Sleep diaries are inexpensive and easy to complete; thus, these tools can be used to collect longitudinal data about sleep. At least one week of sleep monitoring is recommended to capture the variability in sleep. This is particularly important in insomnia because night-to-night variability is large in these patients (Buysse et al., 2010). It is also important to keep in mind that sleep diaries are subjective measures and, therefore, the data they offer are subject to systematic biases. Patients with insomnia particularly tend to overestimate their SOL and WASO and underestimate their TST compared to precise PSG measures (Coates et al., 1982; Means, Edinger, Glenn, \& Fins, 2003a).

Wrist actigraphy detects movement using a motion sensor and stores the data along with clock time and, in some devices, light intensity. The data can be inspected visually or analysed 
using software algorithms that determine sleep parameters including SOL, WASO, TST, and SE. Monitoring is required for a minimum of five days to capture inter-night variability in sleep and wake patterns, but longer recording periods are recommended (Sadeh, 2011). Actigraphy provides a reliable and valid tool for the assessment of sleep-wake patterns in healthy adults with good sleep quality, but the validity of sleep-wake algorithms has been questioned among infants, young children, older adults, and adults with poor sleep quality and fragmented sleep (Insana, Gozal, \& Montgomery-Downs, 2010; Paquet, Kawinska, \& Carrier, 2007; Sitnick, GoodlinJones, \& Anders, 2008). A major problem is that specificity is poor when attempting to distinguish between motionless wakefulness and sleep (Sadeh, 2011). This can be an issue when sleep is interspersed with awakenings (i.e., sleep fragmentation), when patients with insomnia lie awake in bed waiting for sleep, or among patients with low levels of consciousness and tetraparesis—-for example, following TBI (Buysse et al., 2006; Sinclair, Ponsford, \& Rajaratnam, 2014; Zollman, Cyborski, \& Duraski, 2010). The limited accuracy of actigraphy for the detection of wakefulness may affect the calculation of sleep parameters such as SE, sleep time, and WASO. Actigraphy has good correspondence with subjective sleep measures including sleep diary, sleep schedule, and sleep time, but correlations with WASO, night awakenings, and SE are relatively poor (Sadeh, 2011). This may be explained by the inaccuracy of subjective sleep perception and/or because of the inadequate specificity of actigraphy to detect motionless wakefulness. It has been suggested that multi-method simultaneous assessment using sleep diary, actigraphy, and PSG is required to clarify this issue (Sadeh, 2011). It is also important to note that the psychometric properties of actigraphy depend on the type of device and the algorithm it uses, and there has been a lack of consistency in research regarding these properties (Buysse et al., 2006). 
With respect to insomnia assessment, actigraphy measures of SOL, WASO, TST and SE differentiated between 282 good sleepers and 126 individuals with insomnia (Natale, Plazzi, \& Martoni, 2009). The largest PSG-actigraphy validation study $(N=57)$ in insomnia did not detect statistically significant differences between actigraphy and PSG in determining TST, SE, WASO, the number of awakenings, and SOL (Lichstein et al., 2006). Correlations were moderate for the first four measures and weaker for SOL. Other studies have yielded similar results, both in the lab and in the home environment (Sanchez-Ortuno, Edinger, Means, \& Almirall, 2010b; Vallieres \& Morin, 2003). In one study involving older adults with insomnia, actigraphy showed high sensitivity (95.2\%) for sleep but low specificity (36.3\%) in detecting wakefulness, and consequently overestimated total sleep time and SE (Sivertsen et al., 2006). Generally speaking, actigraphy is not indicated for routine insomnia diagnosis, but it can provide valuable information about TST, SE, WASO, and number of awakenings, particularly when patients do not/cannot complete sleep diaries or when subjective data appear to be unrealistic. Another important use of actigraphy is in differential diagnosis between insomnia and CRSWDs. With respect to the research agenda related to actigraphy, it has been recommended that validation be expanded to specific clinical populations and that comparisons between actigraphy and PSG be extended to cover greater periods, both in and out of bed (Sadeh, 2011).

PSG is the gold standard tool for sleep evaluation and it provides the most comprehensive objective assessment of sleep. PSG records EGG, electromyography, electrooculography, and electrocardiography during sleep. Additionally, measures of blood oxygen saturation and respiration are often taken. In certain circumstances, other physiological measures that change during sleep are collected (e.g. temperature, hormonal levels, and oxygen consumption), each of which may be relevant to diagnosis of insomnia but not practical to measure on a routine basis. 
Practice parameters recommend PSG for assessment of insomnia if sleep-related breathing disorders or PLMD are suspected based on the clinical interview, when the initial insomnia diagnosis is uncertain, when treatment fails, or when precipitous arousals occur with violent or injurious behaviour (Littner et al., 2003). Additionally, PSG is useful in detecting potential discrepancies between subjective reports and objective sleep parameters and in insomnia research to determine if a participant meets inclusion or exclusion criteria (Buysse et al., 2006).

The use of PSG has some limitations in insomnia of which it is important to be aware.

First, there is a conditioned association between insomnia and the usual home sleep environment; in other words, individuals with insomnia may sleep better when they sleep in the lab. Alternatively, they may be unusually anxious and uncomfortable in a new environment and, therefore, may have worse sleep than they have at home, especially during the first night (also called the "first night effect"). Secondly, the lab schedule may impose a standard bedtime and rise time and may miss an important part of sleep, especially in patients with sleep initiation or circadian sleep problems who would typically sleep longer in the morning. Thirdly, there is high inter-night variability in sleep among patients with insomnia; therefore, one or two nights may not be sufficient to adequately capture the sleep abnormality (Buysse et al., 2010; Wohlgemuth, Edinger, Fins, \& Sullivan, 1999). Other general drawbacks include the expense of PSG equipment and the need to have a technician present during the night. Home PSG monitoring mitigates most of the above problems since it is conducted in the habitual sleep environment and can be used for longitudinal sleep monitoring. Indeed, there is evidence to suggest that individuals using home PSG do not experience the first night effect and home PSG captures inter-night variability better than lab PSG (Edinger et al., 1997). Home PSG demonstrates the differences between insomnia and good sleep better than laboratory-based PSG and is also 
cheaper than PSG conducted in a sleep laboratory. A disadvantage of home PSG monitoring is that artifacts and data loss may be greater compared to studies conducted in the lab; however, obtaining data from multiple nights can increase validity and statistical power and "make up" for lost data. Another disadvantage is that ambulatory PSG may not encompass video monitoring and, therefore, the scorer and the clinician cannot make behavioural observations.

In summary, insomnia diagnostic assessment includes multiple self-report methods like sleep interview, medical and psychiatric history, and self-report scales. Objective tools such as actigraphy and PSG are not prescribed for routine assessment but are useful in corroborating subjective findings and distinguishing between insomnia and other sleep disorders that either present with insomnia symptoms or are often comorbid with insomnia disorder; for example, OSA, PLMD, and CRSWD. 


\section{Appendix C: Description of Insomnia Assessment Studies that Included Participants with Mild Traumatic Brain Injury}

Despite the high frequency of sleep difficulties reported by patients following mTBI, only a few sleep-focused studies involved pure mTBI samples, see Table 1.4. These studies have used questionnaires to assess "sleep difficulties." Approaches to assessment have included postconcussion checklists with single sleep disturbance items, or multi-item but non-standard questionnaires tapping into insomnia symptoms like difficulty falling asleep or maintaining sleep. Only one recent study has included a validated insomnia screening tool, the Pittsburgh Sleep Quality Index (Theadom et al., 2015).

In a recent, population-based $(N=346)$ longitudinal study in patients with mTBI, poor sleep (Pittsburgh Sleep Quality Index scores $>5$ ) was experienced at rates of approximately $65 \%, 54 \%, 46 \%$, and $41 \%$ of the sample at two weeks, one month, six months, and 12 months post-injury (Theadom et al., 2015). At a higher cut-off for insomnia (Pittsburgh Sleep Quality Index scores $\geq 8$ ), the respective rates were approximately $37 \%, 30 \%, 22 \%$, and $20 \%$ at two weeks, one month, six months, and 12 months post-injury. Although the prevalence of insomnia symptoms remained stable beyond six months, sleep quality further deteriorated in close to $40 \%$ of individuals between six months and 12 months after the injury, while $45 \%$ reported improvement in their sleep quality as indicated by Pittsburgh Sleep Quality Index scores. Furthermore, baseline low sleep quality predicted a range of adverse, self-reported outcomes a year after mTBI, including: poorer cognition, reduced productivity, difficulty with social integration, high endorsement of anxiety, depression, and post-concussion symptoms (Theadom et al., 2015). 
Another study involving a large post-concussion sample $(N=443)$ found that $13.3 \%$ of participants reported sleep disturbance (one item on a post-concussion questionnaire) 10 days after the injury, but this proportion increased to $33.5 \%$ by six weeks post-injury (Chaput, Giguere, Chauny, Denis, \& Lavigne, 2009). Baseline sleep disturbance predicted sleep problems, depressive symptoms, irritability, and headaches at six weeks post-injury.

With regards to the chronic phase of mTBI, $21 \%$ of university students and $32-40 \%$ of high school students from a large sample of participants $(N=3666)$ sustained sleep difficulties following injury (Segalowitz \& Lawson, 1995). In another study $(N=39), 52.6 \%$ of the sample reported sleep initiation difficulties, $53 \%$ had problems with sleep duration, and $65 \%$ reported awakenings from sleep on a detailed sleep questionnaire (Perlis, Artiola, \& Giles, 1997). Patients with a history of TBI also reported more significant sleep difficulties than the orthopedic control group. There is evidence to suggest that the prevalence and severity of insomnia increases with multiple concussions (with a prevalence of 5.6\% in controls, $20.4 \%$ after a single concussion, and 50\% after multiple concussions) after controlling for depression, post-traumatic stress disorder, and concussion symptom severity (Bryan, 2013). Studies involving samples in which the severity of TBI is mixed have also demonstrated rates of insomnia symptoms of 50-75\% among participants, with higher rates observed in individuals with histories of mTBI (Beetar et al., 1996; Clinchot et al., 1998; Collen, Orr, Lettieri, Carter, \& Holley, 2012; Ouellet et al., 2006).

It is important to re-emphasize the difference between insomnia symptoms and insomnia disorder. Insomnia disorder is diagnosed if symptoms meet criteria for duration, frequency, and daytime consequences and if these symptoms are present independent of other sleep disorders. While research has consistently shown that insomnia symptoms are ubiquitous following mTBI 
and that symptoms are more common in mTBI samples than in the general population, only three studies in the TBI sleep literature have used standard diagnostic criteria to distinguish insomnia symptoms from insomnia disorder. Further, these studies have predominantly involved patients with moderate and severe injuries (Fichtenberg, Putnam, Mann, Zafonte, \& Millard, 2001;

Ouellet et al., 2006; Ouellet \& Morin, 2006a).

The aim of the first of these studies was to determine the cut-off score for the Pittsburgh Sleep Quality Index for insomnia in TBI (Fichtenberg et al., 2001). Participants completed the Pittsburgh Sleep Quality Index and sleep diaries and participated in clinical interviews. The authors described that diagnoses of insomnia were based on DSM IV criteria that were operationalized for the purpose of the study. Upon closer inspection, the criteria they set for diagnosis did not fully match the DSM IV definition, mainly because the authors included a sleep duration component (i.e., sleep duration is less than 6.5 hours), which is not in the DSM definition, and did not include the requirement for daytime symptoms attributed to the sleep difficulty. Based on their assessment, 15 of the 50 participants (30\%) were diagnosed with insomnia disorder. Sample characteristics including TBI severity or time since injury were not reported, but one could presume that most participants had moderate or severe injuries because they were all required to participate in neurorehabilitation.

The second study $(N=452)$ assessed insomnia disorder using a detailed questionnaire created for the purpose of the study based on DSM IV, ISCD, and insomnia research diagnostic criteria (Ouellet et al., 2006). According to these standard criteria, 50.2\% of the sample reported insomnia symptoms and $29.4 \%$ of the whole sample met all diagnostic criteria for an insomnia syndrome. The majority $(94.4 \%)$ of participants with insomnia syndrome noted that the insomnia started after the injury. Specifically, they reported that the sleep difficulty appeared days 
(43.2\%), a few weeks (14.4\%), or a few months (28.8\%) following the TBI. Ninety-nine percent of individuals with an insomnia syndrome suffered from chronic insomnia, defined in the study as insomnia enduring at least six months. The average insomnia duration was 6.2 years. More than $80 \%$ had moderate or severe insomnia based on ISI scores. With respect to TBI severity, the majority of the sample had severe TBI and only $13.7 \%$ had mTBI. MTBI and milder injury predicted the presence of insomnia syndrome. Insomnia syndrome was also associated with high pain intensity, depressive symptoms, and fatigue.

While the previous two studies used subjective reports of insomnia symptoms to arrive at diagnosis, the third study also used PSG and compared subjective and objective measures of sleep (Ouellet \& Morin, 2006a). Fourteen individuals were included in the study, four of whom had mTBI and one of whom had mild-to-moderate TBI. All were diagnosed with insomnia based on the Diagnostic Interview for Insomnia (Morin, 1993). Individuals with symptoms of other sleep disorders were excluded. PSG results were consistent with insomnia (i.e., SOL and/or WASO longer than 30 minutes) in 10 patients. There were no statistically significant differences in PSG measures between the insomnia population and the age- and sex-matched control groups, but moderate to large effect sizes (differences between the two groups) were detected in some insomnia measures, including WASO, SE, TST, number of awakenings lasting for five minutes or longer, time spent awake during the night, and stage 1 sleep. When patients taking psychotropic medications were excluded from the analysis, the insomnia group had significantly more arousals from sleep lasting for at least five minutes and significantly shorter REM latencies compared to controls. The latter finding is characteristic of PSG biomarkers of depression and the authors speculated that some patients with insomnia may have been developing depression. 
In summary, research has demonstrated that insomnia symptoms are common in individuals with a history of mTBI and may even be more frequent in patients with mild injuries than in severe TBI. There is also evidence to suggest that $30 \%$ of patients have insomnia disorder - three times the rate that has been observed in the general population. However, this data was obtained from samples in which the majority of individuals demonstrated moderate or severe TBI. The evidence accumulated so far indicates that sleep difficulties and insomnia disorder in the first weeks/months after injury predict the presence of persistent post-injury (postconcussion) symptoms (Chaput et al., 2009; Ouellet et al., 2006; Theadom et al., 2015). This is an important issue not only with respect to quality of life and recovery but also with regards to litigation and financial compensation (Ontario Neurotrauma Foundation, 2013). Insomnia is the major obstacle to community reintegration in Ontario workers with delayed recovery from mTBI (Mollayeva, Shapiro, Mollayeva, Cassidy, \& Colantonio, 2015). In spite of the evidence showing the clinical significance of insomnia symptoms among individuals with a history of mTBI, there have not been any studies using proper diagnostic criteria and conducting a diagnostic assessment in a homogenous sample of patients with a mTBI. 


\section{Appendix D: Assessment Tools and Methods}

\section{Laboratory Polysomnography (PSG)}

XLTek EEG-Polysomnigraphy system was used to record PSG in the sleep laboratory.

The following montage was used to perform the tests:

\begin{tabular}{|c|c|c|c|c|c|}
\hline $\begin{array}{l}\text { Channel } \\
\text { Name }\end{array}$ & Channel Type & Frequency & $\begin{array}{l}\text { Gain } \\
(\mu \mathrm{V} / \mathrm{mm})\end{array}$ & $\begin{array}{l}\text { High Filter } \\
\text { in } \mathbf{H z}\end{array}$ & $\begin{array}{l}\text { Low Filter } \\
\text { in } \mathrm{Hz}\end{array}$ \\
\hline$F 4-M 1$ & $E E G$ & $200 \mathrm{~Hz}$ & 35 & 0.3 & \\
\hline$F 3-M 2$ & $E E G$ & $200 \mathrm{~Hz}$ & 35 & 0.3 & \\
\hline$C 4-M 1$ & $E E G$ & $200 \mathrm{~Hz}$ & 10 & 35 & 0.3 \\
\hline C3-M2 & $E E G$ & $200 \mathrm{~Hz}$ & 10 & 35 & 0.3 \\
\hline$O 2-M 1$ & $E E G$ & $200 \mathrm{~Hz}$ & 10 & 35 & 0.3 \\
\hline$O 1-M 2$ & $E E G$ & $200 \mathrm{~Hz}$ & 10 & 35 & 0.3 \\
\hline$L-E O G$ & Ocular, Left & $200 \mathrm{~Hz}$ & 10 & 35 & 0.3 \\
\hline$R-E O G$ & Ocular, Right & $200 \mathrm{~Hz}$ & 10 & 35 & 0.3 \\
\hline CHIN & EMG, Sub-mental & $200 \mathrm{~Hz}$ & 10 & 100 & 10 \\
\hline$E C G$ & $E C G$ & $200 \mathrm{~Hz}$ & 70 & 70 & 1 \\
\hline$R-T I B$ & Legs, Right & $200 \mathrm{~Hz}$ & 100 & 100 & 10 \\
\hline$L-T I B$ & Legs, Left & $200 \mathrm{~Hz}$ & 100 & 100 & 10 \\
\hline FLOW & Airflow & $200 \mathrm{~Hz}$ & & 30 & 0.1 \\
\hline CHEST & Chest & $200 \mathrm{~Hz}$ & & 30 & 0.1 \\
\hline$A B D O M E N$ & Abdomen & $200 \mathrm{~Hz}$ & & 30 & 0.1 \\
\hline $\begin{array}{l}\text { NASAL } \\
\text { PRESS }\end{array}$ & Nasal & $200 \mathrm{~Hz}$ & $\begin{array}{c}\text { According } \\
\text { to the } \\
\text { manufactu- } \\
\text { rerer }\end{array}$ & & \\
\hline$B-P O S^{*}$ & Body Position & $200 \mathrm{~Hz}$ & & & \\
\hline $\mathrm{SaO} 2$ & $\mathrm{SaO}_{2}$ & $200 \mathrm{~Hz}$ & & & \\
\hline
\end{tabular}


Oro-nasal thermal sensor (thermistor/thermocouple) was used to detect nasal airflow in order to determine if there is a cession of airflow (apnea). Nasal pressure transducer detected hypopneas. Piezoelectric belts were used to detect respiratory effort. Blood oxygen saturation was monitored with pulse oximeter with a maximum acceptable signal averaging time of 3 seconds. Manual scoring of sleep staging and events followed the current standard guidelines (AASM, 2016). The scoring followed the following stages: (1) sleep staging; (2) arousal detection; (3) respiration analysis; (4) limb movement analysis; (6) ECG analysis; (7) adjustment of the body position as required; (8) review of the video-recording; (9) other findings.

\section{Home Polysomnography}

Compumedics Sompté ( ) (Victoria, Australia) PSG device recorded full PSG in the home of the participants. PSG allowed the continuous collection of physiologic data during sleep, including EEG, electrooculogram (EOG) and submental electromyogram (EMG). Additionally, electro-cardiogram (ECG), respiratory movements, limb movements, and blood oxygen saturation were recorded. Participants attached skin electrodes to the following standard positions: Fp1 and Fp2, $\mathrm{C} 3$ and $\mathrm{C} 4, \mathrm{O} 1$ and $\mathrm{O} 2$, and $\mathrm{T} 3$ and $\mathrm{T} 4$. Submental EMG was recorded with one electrode placed underneath the chin and ECG was conducted with two electrodes attached to the chest. Respiratory effort was recorded with piezoelectric thoracic and abdominal belts. Airflow was measured using nasal pressure transducer. A pulse oximeter was used for measurement of oxygen saturation. Surface EMG electrodes placed over the tibialis anterior recorded EMG. A body posture sensor recorded body position during sleep. PSG data was sent for analysis via secure cloud technology.

The recordings were subjected to automated analysis by Esprit Nova TM (Neurozone MSH Inc. Dundas, ON, Canada), the latest generation of sleep diagnostic tools. Esprit Nova ${ }^{\mathrm{TM}}$ can 
analyze laboratory and home sleep studies without a technologist and provide all the parameters related to the PSG in a spreadsheet form, which serves directly as an input to statistical packages. For the purpose of the current study, the automatic scoring was conducted according to the AASM standard scoring criteria (AASM, 2016). Two sleep technologists with 25 years scoring experience checked each record for accuracy of scoring. In addition to the standard scoring, micro-architectural sleep analysis determined a measure of the distance (level of consciousness) between N2 and REM sleep.

\section{Wrist Actigraphy}

Participants wore a Philips Respironics Actiwach on their non-dominant wrists for 12-14 consecutive 24-hour periods, starting on the date of their first study visit. The device contains a solid piezoelectric accelerometer with $0.35-7.5 \mathrm{~Hz}$ bandwidth and $0.5-2 \mathrm{G}$ peak value. The actiwatch was set to record 30-second epochs at medium sensitivity to detect wake threshold (40 activity counts per minute). Data was downloaded to Actiware 6.0.7 software and scored both automatically and manually (i.e., the rest periods were set manually and software algorithms automatically calculated sleep parameters).

\section{Dim Light Melatonin Onset Test (DLMO Test)}

Participants completed the MEQ after they arrived for the DLMO test. Eight saliva samples were collected from each participant using a swab according to standard procedures (Buhlmann EK DSM @ Saliva Melatonin ELISA kit; Buhlmann Laboratories, Switzerland). The first three or four (baseline) samples were taken at every 30 minutes and the remining samples were collected hourly. When individuals had a sleep schedule in the normal circadian phase (based on their sleep diary data), the first sample was taken by $6 \mathrm{pm}$ and the last sample was collected at $12 \mathrm{am}$. For individuals with delayed sleep schedule, the sample collection was 
extended to 1 or $2 \mathrm{am}$. In individuals with advanced sleep schedule, the first sample was collected a minimum of six hours before their average habitual bedtime and the last sample was taken at $11 \mathrm{pm}$. Melatonin samples were frozen and analyzed using enzyme-linked immunosorbent assay (ELISA) as per manufacturer’s instructions (Buhlmann EK DSM @ Saliva Melatonin ELISA kit; Buhlmann Laboratories, Switzerland) in the Sick Children's Hospital and in the Melatonin Assessment and Testing Lab in Toronto, ON, Canada. Duplicates of each saliva sample were analyzed (one undiluted and one diluted 1:4 within the range of linearity as instructed in the manufacturer's protocol). For each data point, the values were averaged to increase precision. The diluted samples were used to determine melatonin values above the upper threshold of functional sensitivity $(20.5 \mathrm{pg} / \mathrm{ml})$ to increase the accuracy at higher concentrations, as per manufacturer's instructions. The intra-assay and inter-assay coefficiences of variation in this study were 7.62 and 8.88 .

As per the consensus guidelines, DLMO was determined as the clock time, when the melatonin concentration reached and remained above the threshold (two $S D$ above the baseline or, when there was less then 3 baseline values, an absolute value of $3 \mathrm{pg} / \mathrm{ml}$; (Benloucif et al., 2008).

\section{Interviews}

Duke Structured Interview for Sleep Disorders ( Edinger, Kirby, A., Lineberger, M., Loiselle, M., Wohlgemuth, W., \& Means, M., 2004). The Duke Structured Interview for Sleep Disorders (DSISD) is dived into four modules assessing insomnia disorder, sleep disorders associated with excessive sleepiness and hypersomnia, CRSWDs, and parasomnias according to DSM IV and ICSD 2 criteria. The study used an updated version of the DSISD in which the insomnia section has been modified to match the new DSM 5 criteria. The original DSISD 
possessed adequate inter-rater reliability for most insomnia subtypes $(\kappa=.42-71)$ except paradoxical insomnia and inadequate sleep hygiene insomnia $(\kappa=.20)$ (Carney et al., 2008). Since the DSM 5 does not distinguish between different types of insomnia, the inter-rater reliability is higher for the DSM 5 insomnia diagnosis (Edinger, Wohlgemuth, Wyatt, \& Krystal, 2015).

Structured Clinical Interview for DSM-5 Axis I Disorders - Clinician Version (First, 2002). The Structured Clinical Interview for DSM-5 Axis I Disorders - Clinician Version (SCID-5 CV) is a semi-structured interview for diagnosing Axis I disorders according to DSM 5 criteria. It was used in the study to assess for the presence of comorbid disorders and to exclude participants whose symptoms meet exclusion criteria. Kappa values for both test-retest and interrater reliability were high for the previous version of the SCID (SCID-IV). The SCID 5 was released in October 2015, and reliability values have not been published.

\section{Consensus Sleep Diary (Carney et al., 2012)}

The Consensus Sleep Diary was developed to provide a standard sleep log based on patients' input and expert consensus. Participants entered the time they went to bed, the time when they first tried to get to sleep, the time it took them to fall asleep, the number of awakenings they experienced during the night (excluding the final awakening), the total duration of nighttime awakenings, the time of their final awakening, the time they spent in bed between their final awakening and rising time, the time when they got out of bed, and their estimated total sleep time. They also recorded whether they woke up earlier than desired and, if yes, how much earlier. They entered information about alcohol use, consumption of caffeinated beverages, hypnotic use, and naps on the previous day. In one study the sleep indices in the Consensus Sleep Diary differentiated between individuals with insomnia and good sleepers and were 
significantly correlated with the ISI and with actigraphy measures (Maich, Lachowski, \& Carney, 2016).

\section{Self-report Scales}

Anxiety and Preoccupation about Sleep Questionnaire (APSQ; Tang \& Harvey, 2004). The APSQ was developed to measure the subjective intensity of sleep-specific worry in insomnia (Tang \& Harvey, 2004). The original scale consists of 10 items; respondents are asked to rate, on a 10-point scale, how true each statement has been for them in the past month.

Consistent with the cognitive model of insomnia, the APSQ assesses worry both about sleep and about the daytime consequences of poor sleep. The modified version of the APSQ (using a fivepoint rather than 10-point response scale) yielded a two-factor structure (worries about the consequences of poor sleep and about the uncontrollability of sleep) based on data obtained from a large $(N=2333)$ community sample (Jansson-Frojmark, Harvey, Lundh, Norell-Clarke, \& Linton, 2011). The internal consistency of both subscales was high $(\alpha=.91$ and $\alpha=.86)$. APIC scores were significantly higher in participants with insomnia than in poor sleepers and were the lowest in good sleepers. The scores were moderately correlated with self-reported SOL, WASO, early morning awakening, TST, and daytime impairment. The scale is sensitive to treatmentrelated change in worry (Harvey, Sharpley, Ree, Stinson, \& Clark, 2007).

The Assessment Acceptability Questionnaire was developed for the dissertation study to inquire about participants' experience of the assessment methods and their suggestions for how to make the assessments the least burdensome. The items included: (1) broad, open ended questions, for example: "Thinking of the study as a whole, what comments (negative or positive) do you have regarding the study?"; (2) focused, open ended questions, for example: "How could we make the above assessments less burdensome?"; (3) Likert-type scales of the ease of use the 
assessment tools and the degree of tolerability of the various assessment methods; and (4) one forced choice item of the preference between laboratory and home PSG.

Daytime Insomnia Symptom Response Scale (Carney, A. L. Harris, A. Falco, \& J. D. Edinger, 2013). The Daytime Insomnia Symptom Response Scale (DISRS) assesses rumination about the daytime symptoms of insomnia, including decreased motivation, low mood, and tiredness. Respondents indicate the frequency with which they endorse the scale's 20 items when they are feeling down or tired. Total scores range from 20 to 80, with higher scores indicating more intense rumination. The DISRS was developed in a sample of undergraduate students and in adults with comorbid insomnia and depression (Carney et al., 2013). The construct validity of the DISRS is supported by: (a) its correlation with measures of insomnia severity, fatigue, and general rumination; (b) significantly higher scores in poor sleepers than in good sleepers; (c) the significant, independent predictive value of the DISRS in terms of insomnia severity; and (d) separate factor loadings of rumination and worry items (Carney et al., 2013). The scale has good internal consistency in both nonclinical and clinical samples ( $\alpha=.93$ and .94 respectively). A subsequent study showed that the DISRS mediated the relationship between insomnia severity and fatigue, suggesting that preoccupation with fatigue is a psychopathological factor that contributes to insomnia-related fatigue (Harris, Zalai, Moss, \& Carney, 2016).

Depression Anxiety Stress Scale-21 (DASS-21; Lovibond \& Lovibond, 1995). The DASS-21 is a 21-item questionnaire assessing symptoms of depression, anxiety, and stress (Lovibond \& Lovibond, 1995). Specifically, the depression subscale contains items that refer to low mood, the anxiety subscale assesses symptoms of psychophysiological arousal, and the stress subscale captures the signs of overlapping tension/irritability that are components of both depression and anxiety (Antony et al., 1998). There is evidence that the items from the three 
subscales load onto a general negative affect factor; subscale scores may be summed to calculate this value (Henry \& Crawford, 2005). According to the standard instructions, scores from each subscale are added and multiplied by two, which yields a total subscale score ranging from 0 to 42. The depression subscale scores differentiate between mild (scores of 10 to 13), moderate (14 to 20 ), severe (21 to 27 ), and extremely severe (above 28 ) depression symptoms. With respect to anxiety, scores differentiate between mild (scores of 8 to 9), moderate (10 to 14 ), severe (15 to 20), and extreme (above 20) anxiety. On the stress subscale, scores differentiate between mild (15 to 18 ), moderate (19 to 25 ), severe (26 to 33 ), and extremely severe (34) stress. The DASS21 demonstrates good internal consistency (alpha coefficients of .94 for the depression subscale, .92 for the anxiety subscale, and .95 for the stress subscale). Convergent validity measures indicate good construct validity in clinical samples (i.e., the correlation between the depression subscale and the Beck Depression Inventory (BDI) was .79; the correlation between the anxiety subscale and the Beck Anxiety Inventory (BAI) was .85; the correlation between the stress scale and BDI, State-Trait Anxiety Inventory- Trait subscale, and BAI were .69, .68, 70, respectively) (Antony et al., 1998). The DASS-21 was chosen to assess depression and anxiety in the current study because the depression subscale does not contain somatic items and the DASS reliably differentiates between anxiety and depression (Antony et al., 1998). Mood and anxiety symptoms are often associated with insomnia and are also common after mTBI; their association with subjective sleep quality and feeling rested upon awakening were calculated in the proposed study.

Dysfunctional Beliefs and Attitudes about Sleep Scale (DBAS-16; Morin et al., 2007). The DBAS-16 was derived from the original 30-item DBAS to assess beliefs and attitudes that 
maintain chronic insomnia (Morin, 1994; Morin et al., 2007) The 16 items are statements that tap into causal attributions, beliefs about negative consequences of insomnia, unrealistic expectations about sleep, perception of low control and predictability of sleep, and dysfunctional beliefs about sleep-promoting behaviours. There is no specific recall period. The response choices range on a continuous scale from 0 ("Strongly disagree") to 10 ("Strongly agree"). In the original development study, the DBAS-16 showed an internal consistency of 0.79 , a testretest reliability of $r=0.89$ for a two-week interval, and adequate convergent validity with the Insomnia Severity Index, a sleep diary, and PSG (Morin et al., 2007).

Epworth Sleepiness Scale (ESS; Johns 1991). Fatigue and sleepiness are conceptually separate conditions and distinguishing between them is important both in research and in clinical settings. The ESS was used in the current study to identify individuals with subjective excessive daytime sleepiness. The ESS lists eight life situations and asks respondents to mark on a scale the likelihood with which they would fall asleep in these situations. Summary scores range from 0 to 24 . A score above 10 indicates excessive subjective daytime sleepiness.

Fatigue Severity Scale (FSS; Krupp, 1989). The FSS measures fatigue-related functional impairment over the past two weeks (Krupp et al., 1989). The FSS is a valid and reliable measure of fatigue in chronic fatigue syndrome, cancer, MS, sleep disorders, major depressive disorder, chronic hepatitis C infection, Parkinson's disease, brain injury, and amyotrophic lateral sclerosis (Dittner, Wessely, \& Brown, 2004). When completing the questionnaire, respondents use a Likert scale ranging from 1 to 7 to indicate the degree to which they agree with nine statements about the impact of fatigue. The item scores are summed and then divided by 9 to calculate the average fatigue score. An average score equal to or greater than 4 indicates higher than normal 
fatigue. Fatigue was measured as part of the study because it is the leading daytime symptom associated with insomnia; it is also a common complaint following TBI.

Glasgow Sleep Effort Scale (GSES; Broomfield \& Espie, 2005). The GSES was developed to assess sleep effort based on the attention-intention-effort model of insomnia (Broomfield \& Espie, 2005). The scale consists of seven statements and respondents indicate whether the statements were "very much," "to some extent," or "not at all" true for them during the past week. The scale demonstrated adequate internal consistency $(\alpha=.77)$ during development in a clinical insomnia sample and it moderately correlated with the DBAS. Furthermore, it correctly identified $92 \%$ of insomnia patients and $87.5 \%$ of good sleepers at a cut-off score of 2 (Broomfield \& Espie, 2005). The GSES also predicted insomnia severity in a recent study involving patients with primary insomnia, but it did not predict PSG-measured TST (Hertenstein et al., 2015). The GSES was significantly associated with sleep disturbance (Pittsburgh Sleep Quality Index) scores in an outpatient depression and anxiety sample after controlling for negative affect (Fairholme \& Manber, 2014).

Insomnia Severity Index (ISI; Morin, 1993). The ISI was developed to measure the subjective severity of insomnia symptoms and has been recommended by an expert panel for use in insomnia research (Buysse et al., 2006; Morin, 1993a). The degree of insomnia severity is determined by the summary scores of seven items as follows: 0 to 7 means no clinical insomnia; 8 to 14 indicates "sub-threshold" insomnia; 15 to 21 is moderate insomnia; and 22 to 28 is severe insomnia. The questionnaire has good internal consistency and convergent validity with sleep diary, sleep onset latency on PSG, and the global score on the BBAS (Bastien, Vallieres, \& Morin, 2001). A summary score of 11 has been recommended as a cut-off score in insomnia research. This score identified participants within a clinical insomnia sample with $97.2 \%$ 
sensitivity and $100 \%$ specificity (Morin, Belleville, Belanger, \& Ivers, 2011). The ISI was used in the study to select individuals with clinically significant insomnia symptoms for study participation.

Morningness-Eveningness Scale (MEQ). The MEQ was developed to assess individual circadian phase preferences (Horne \& Ostberg, 1976). The questionnaire consists of 19 items that inquire preferences for bedtime, wake up time, sleep time, cognitive and physical activities, and work, in addition to assessing dependence on alarm clock, morning appetite and tiredness. Respondents are asked to indicate preferences based on their "feeing best" times. Summarized scores range between 18 and 65, with higher scores suggesting morning chronotype and lower scores indicating evening chronotype. When used as a categorical measure, scores 16-30 suggest “definite evening"; 31-41 "moderate evening"; 42-58 "intermediate"; 59-69 "moderate morning" and 70-86 "definite morning” circadian preference. These cut-off scores were determined based on data obtained from the validation sample of 18-32-year-old students. Data from a larger population-based sample of middle-aged individuals showed that scores below 53 were evening types, whereas people obtaining scores above 64 were morning types. In the current study, the original cut-offs cores were used among participants between 17 and 32, and the cut-offs established for middle-age adults were used among participants above 32. The MEQ summary score moderately correlates with DLMO $(r=.70)$ in healthy, young, good sleepers (Taillard, Philip, Chastang, \& Bioulac, 2004).

Visual Analogue Scale for Sleep Quality (VAS-SQ). Participants completed a singleitem sleep quality scale in the mornings for two consecutive weeks. They completed the VASSQ instead of sleep diary sleep quality ratings because the five items on the sleep diary restrict the range of responses and, making correlations difficult to calculate. Participants were asked to 
draw an $\mathrm{X}$ on a 100-millimeter horizontal line to mark the degree of the perceived quality of the previous night sleep. The scale ranged between two ends of a continuum of sleep quality, with an anchor on the left end labeled "extremely poor sleep quality" and an anchor on the right labeled "excellent sleep quality." The VAS-SQ was scored by measuring the distance between the left end and the point where the $\mathrm{X}$ bisects the horizontal line (e.g. a bisection at $73 \mathrm{~mm}$ from the left will be assigned a score of 73). It has been recommended that daily, subjective measures be used in insomnia research due to the variability in sleep and arousal in this patient group. The VAS is especially useful for repeated assessments because it is sensitive to change (Steiner \& Norman, 2008).

Visual Analogue Scale for Restorative Sleep (VAS-RS). The VAS-RS was used to ask participants to rate how rested/refreshed they feel when waking up in the morning. The anchor on the left was labeled "not at all rested/refreshed" and the one on the right was be labeled "very well rested/refreshed." Participants were asked to place an X on this $100 \mathrm{~mm}$ horizontal line. Ratings were scored by measuring the distance between the left end and the point where the $\mathrm{X}$ bisects the horizontal line (e.g. bisection at $50 \mathrm{~mm}$ will be assigned a score of 50).

Visual Analogue Scale for Pain (VAS-P). Participants marked their pain level every night before they went to bed on a 100-mm visual analogue scale. The scale was anchored so that the left end indicated "no pain" and the right end marked "extreme pain." A daily pain scale was used because pain and, in particular, headache is very common after TBI and may interfere with sleep or cause EEG arousals.

Rivermead Post-Concussion Symptom Questionnaire (RPQ; King et al, 1995). The RPQ assesses 16 symptoms that patients commonly report after concussion. Participants rated the extent to which they endorsed each symptom (as compared to before the accident). The 
Likert-type response scales range from 0 ("Not experienced at all") to 4 ("A severe problem").

The RPQ has been recommended for the assessment of post-concussion symptoms by the Ontario Neurotrauma Foundation (2013).

STOP-BANG Questionnaire. The STOP-BANG questionnaire is a self-administered screening tool for obstructive sleep apnea (Chung et al., 2008). The questionnaire includes seven yes/no questions and a scale where respondents can mark their height and body weight, which is also converted to a positive/negative answer. The questionnaire was validated with pre-surgical patients. A cut-off of three "yes" answers showed a sensitivity of $84 \%, 93 \%$ and $100 \%$ for identifying individuals with apnea-hypopnea indices (AHI) above five, 15 and 30 respectively. The specificity of scores three, four, five, six, seven, eight were $40.3 \%, 60.6 \%, 79.7 \%, 91.5 \%$, $97.5 \%$ and $98.5 \%$ in the pre-surgical population. The cut-off of three was suggested in populations with a high risk for sleep apnea because of the high sensitivity at this cut-off. On the other hand, the cut-off of five is recommended if one wants to achieve high specificity to detect moderate and severe sleep apnea (Chung et al., 2012).

In sleep clinic populations, the sensitivity of the STOP-BANG questionnaire is $85 \%$ to detect sleep apnea, while it has a specificity of $49 \%$ at the score of $\geq 3$. A meta-analysis suggested that a cut-off score of 3 is used at sleep clinics to exclude patients who do not have apnea (scores 0-2) and the cut-off score of 5 is used to identify patients with moderate or severe sleep apnea (Nagappa et al., 2015). 


\section{Appendix E: Circadian Sleep Regulation and the Effect of Melatonin on Sleep}

Human sleep-wake cycles are regulated by the synergistic activity of homeostatic system (Process S) and the circadian system (Process C). In the homeostatic process, sleep debt accumulates during wake hours and dissipates during sleep. The strength of the homeostatic drive is marked by non-rapid eye movement slow wave sleep activity on the sleep EEG and by theta activity on the wake EEG. The sleep homeostat oscillates within a set band: when it reaches its upper limit, sleep is initiated and the sleep drive gradually declines; when it reaches its lower boundary, the individual wakes up and sleep drive starts to accumulate.

Process $\mathrm{C}$ is regulated by the central pacemaker in the suprachiasmatic nucleus and by peripheral oscillators. The oscillation of the suprachiasmatic pacemaker is an autonomous process, creating internal rhythms harmonized with the $24 \mathrm{~h}$ solar day. The internal clock is synchronized with the solar time through environmental cues, also called "zeitgebers". The most potent zeitgeber is light that is captured by photosensitive ganglion cells in the retina. These cells send signals (glutamate, substance $\mathrm{P}$, and pituitary adenyl cyclase-activating peptide) to the suprachiasmatic nucleus where these transmitters affect the expression of clock genes. The output of the "master clock" synchronize sleep and wakefulness, core body temperature, food intake and metabolism, as well as immune and endocrine functions so that their oscillations are aligned with each other and are in harmony with the environment.

With respect to sleep and wakefulness cycles, the circadian system promotes wakefulness during the biological day and thus counteracts the accumulating homeostatic drive for sleep. In the biological evening, when the sleep drive from the homeostat is high, the alerting effect of the circadian system switches off. In the properly entrained system, the biological evening occurs when the environmental light intensity decreases, and under dim light conditions (i.e., 
environmental dusk), the suprachiasmatic nucleus's inhibitory output on the pineal gland declines, which permits the secretion of melatonin from the pinealocytes. Sleep onset occurs approximately 2-3 hours after melatonin starts to rise (dim light melatonin onset or DLMO). Melatonin level increases during the night and peaks early in the morning, typically between 2 am and 5 am (melatonin acrophase). The endogenous melatonin level drops to the low, baseline daytime level (dim light melatonin offset) in the biological morning after the morning habitual wake time and the cycle of wakefulness and sleep to starts over.

Melatonin is called the "hormone of the dark", because it is produced in darkness in all mammals and its secretion is supressed by light. With respect to its effects on sleep, melatonin has "chrono-hypnotic" properties: it exerts a strong chrono-biotic effect (i.e., it marks internal time and affects the timing of sleep and wakefulness) and it also has modest hypnotic properties, especially in higher doses.

According to the professional consensus guidelines, the standard melatonin assessment method for clinical and most research purposes is collecting saliva samples in regular 30 or 60minute intervals under dim light ( $<30$ lux or - using more strict criteria $-<10$ lux $)$ conditions (for detailed description, please see Dim Light Melatonin Onset test in Appendix D). DLMO is the most reliable marker of the internal circadian phase. It is defined as the clock time when the melatonin concentration surpasses and stays above a standard threshold (two $S D$ above the daytime baseline; or above $3 \mathrm{pg} / \mathrm{ml}$ in saliva samples; or above $10 \mathrm{pg} / \mathrm{ml}$ in serum samples).

CRSWD arise if there is a malfunction of the circadian system; the individual's circadian oscillation is not entrained with the environment; or if there is a misalignment between the circadian propensity for sleep and the individually/socially desired sleep-wake pattern. If the individual tries to sleep when the circadian drive for sleep is low and tries to stay awake when 
the circadian pressure for sleep is high, they may experience: (1) symptoms of insomnia (i.e. difficulty with sleeping when one wants or "should" sleep); (2) sleep episodes that occur at "nonconventional" or socially undesirable times; (3) excessive daytime sleepiness; (4) impaired alertness when one is expected to be alert and engage in social activities; (5) clinically significant distress concerning one's sleep and wake pattern; and/or (6) functional impairment. A general definition of CRSWD is in panel 1.4 and the CRSWD that are relevant to this dissertation are summarized in Table 5.2. 


\section{Appendix F - PSG Sleep Parameters}

Descriptive Statistics of Polysomnography Indices

\begin{tabular}{|c|c|c|c|c|c|c|c|c|c|}
\hline \multicolumn{4}{|c|}{ LAB } & \multicolumn{3}{|c|}{ 1st $^{\text {sIGHT HOME }}$} & \multicolumn{3}{|c|}{$2^{\text {nd }}$ NIGHT HOME } \\
\hline & $\begin{array}{l}\text { Mean } \\
(\mathrm{SD})\end{array}$ & $95 \% \mathrm{CI}$ & $\begin{array}{l}\text { Median } \\
\text { (IR) }\end{array}$ & Mean (SD) & $95 \% \mathrm{CI}$ & $\begin{array}{c}\text { Median } \\
\text { (IR) }\end{array}$ & Mean (SD) & $95 \% \mathrm{CI}$ & $\begin{array}{c}\text { Median } \\
(\text { IR })\end{array}$ \\
\hline $\mathrm{TIB}(\min )$ & $\begin{array}{l}442.68 \\
(41.55)\end{array}$ & $\begin{array}{l}428.65- \\
456.02\end{array}$ & $\begin{array}{l}435.50 \\
(38.68)\end{array}$ & $\begin{array}{l}481.22 \\
(96.65)\end{array}$ & $\begin{array}{c}448.69- \\
520.29\end{array}$ & $\begin{array}{c}470.00 \\
(120.15)\end{array}$ & $\begin{array}{c}479.99 \\
(122.44)\end{array}$ & $\begin{array}{l}436.07- \\
522.43\end{array}$ & $\begin{array}{c}470.00 \\
(146.65)\end{array}$ \\
\hline $\mathrm{TST}(\min )$ & $\begin{array}{l}353.59 \\
(80.96)\end{array}$ & $\begin{array}{l}323.79- \\
380.54\end{array}$ & $\begin{array}{l}380.25 \\
(82.13)\end{array}$ & $\begin{array}{l}366.02 \\
(79.81)\end{array}$ & $\begin{array}{c}340.30- \\
391.49\end{array}$ & $\begin{array}{c}360.00 \\
(100.88)\end{array}$ & $\begin{array}{c}383.83 \\
(103.50)\end{array}$ & $\begin{array}{c}347.32- \\
418.90\end{array}$ & $\begin{array}{c}382.25 \\
(132.13)\end{array}$ \\
\hline SE (percent) & $\begin{array}{c}81.41 \\
(13.27)\end{array}$ & $\begin{array}{l}76.66- \\
85.37\end{array}$ & $\begin{array}{c}84.80 \\
(18.10)\end{array}$ & $\begin{array}{c}79.28 \\
(14.17)\end{array}$ & $\begin{array}{l}74.20- \\
84.32\end{array}$ & $\begin{array}{c}84.80 \\
(19.30)\end{array}$ & $\begin{array}{c}83.61 \\
(10.88)\end{array}$ & $\begin{array}{l}79.88- \\
87.14\end{array}$ & $\begin{array}{c}86.70 \\
(14.65)\end{array}$ \\
\hline $\mathrm{N} 1(\min )$ & $\begin{array}{c}43.29 \\
(29.21)\end{array}$ & $\begin{array}{l}32.47- \\
54.11\end{array}$ & $\begin{array}{c}35.50 \\
(44.50)\end{array}$ & $\begin{array}{c}26.61 \\
(14.59)\end{array}$ & $\begin{array}{c}16.97- \\
26.40\end{array}$ & $21(22)$ & $\begin{array}{c}20.03 \\
(16.17)\end{array}$ & $\begin{array}{l}14.60- \\
26.17\end{array}$ & $15(22)$ \\
\hline N1(percent) & $\begin{array}{l}11.82 \\
(8.73)\end{array}$ & $\begin{array}{l}9.21- \\
14.42\end{array}$ & $9.7(8.8)$ & $5.93(4.17)$ & $4.54-7.38$ & $6.00(6.73)$ & $5.56(4.23)$ & 4.11-7.03 & $4.75(6.60)$ \\
\hline $\mathrm{N} 2$ (min) & $\begin{array}{l}204.82 \\
(51.77)\end{array}$ & $\begin{array}{c}189.62- \\
220.03\end{array}$ & $\begin{array}{c}200 \\
(62.50)\end{array}$ & $\begin{array}{l}186.19 \\
(63.14)\end{array}$ & $\begin{array}{c}165.44- \\
206.95\end{array}$ & $\begin{array}{c}181 \\
(101.50)\end{array}$ & $\begin{array}{l}184.84 \\
(70.40)\end{array}$ & $\begin{array}{l}161.70- \\
207.98\end{array}$ & $\begin{array}{c}192.50 \\
(115.13)\end{array}$ \\
\hline $\mathrm{N} 2$ (percent) & $\begin{array}{c}56.75 \\
(10.93)\end{array}$ & $\begin{array}{r}53.61 \\
-59.89\end{array}$ & $\begin{array}{c}57.10 \\
(15.40)\end{array}$ & $\begin{array}{c}47.32 \\
(14.89)\end{array}$ & $\begin{array}{c}42.49- \\
52.15\end{array}$ & $49(18.80)$ & $\begin{array}{c}48.84 \\
(15.94)\end{array}$ & $\begin{array}{c}43.67- \\
54.01\end{array}$ & $\begin{array}{c}48.60 \\
(17.10)\end{array}$ \\
\hline $\mathrm{N} 3(\min )$ & $\begin{array}{c}45.49 \\
(31)\end{array}$ & $\begin{array}{c}36.69- \\
54.46\end{array}$ & $\begin{array}{c}38.50 \\
(50.20)\end{array}$ & $\begin{array}{c}75.08 \\
(35.04)\end{array}$ & $\begin{array}{c}63.47- \\
87.00\end{array}$ & $\begin{array}{c}79.00 \\
(57.00)\end{array}$ & $\begin{array}{c}84.58 \\
(50.67)\end{array}$ & $\begin{array}{l}68.13- \\
101.19\end{array}$ & $\begin{array}{c}78.50 \\
(78.50)\end{array}$ \\
\hline N3 (percent) & $\begin{array}{c}15.30 \\
(11.20)\end{array}$ & $\begin{array}{c}12.08- \\
18.52\end{array}$ & $\begin{array}{c}12.70 \\
(13.30)\end{array}$ & $\begin{array}{c}18.39 \\
(11.68)\end{array}$ & $\begin{array}{c}14.83- \\
21.94\end{array}$ & $\begin{array}{c}20.85 \\
(15.93)\end{array}$ & $\begin{array}{c}19.34 \\
(14.86)\end{array}$ & $\begin{array}{c}14.77- \\
23.92\end{array}$ & $17(21)$ \\
\hline
\end{tabular}


Table Appendix F (cont.)

\begin{tabular}{|c|c|c|c|c|c|c|c|c|c|}
\hline \multicolumn{4}{|c|}{ LAB } & \multicolumn{3}{|c|}{$1^{\text {st }}$ NIGHT HOME } & \multicolumn{3}{|c|}{$2^{\text {nd }}$ NIGHT HOME } \\
\hline & $\begin{array}{c}\text { Mean } \\
(\mathrm{SD})\end{array}$ & $95 \% \mathrm{CI}$ & $\begin{array}{l}\text { Median } \\
\text { (IR) }\end{array}$ & Mean (SD) & $95 \% \mathrm{CI}$ & $\begin{array}{l}\text { Median } \\
\text { (IR) }\end{array}$ & Mean (SD) & $95 \% \mathrm{CI}$ & $\begin{array}{c}\text { Median } \\
\text { (IR) }\end{array}$ \\
\hline REM (min) & $\begin{array}{c}58.21 \\
(34.86)\end{array}$ & $\begin{array}{c}48.09- \\
68.33\end{array}$ & $\begin{array}{c}66 \\
(53.38)\end{array}$ & $\begin{array}{c}87.26 \\
(49.01)\end{array}$ & $\begin{array}{l}71.15- \\
103.34\end{array}$ & $\begin{array}{c}81 \\
(58.13)\end{array}$ & $\begin{array}{c}83.06 \\
(57.04)\end{array}$ & $\begin{array}{l}64.31- \\
101.81\end{array}$ & $\begin{array}{c}81.25 \\
(61)\end{array}$ \\
\hline REM (percent) & $\begin{array}{l}16.32 \\
(7.60)\end{array}$ & $\begin{array}{c}14.14- \\
18.50\end{array}$ & $\begin{array}{c}18.30 \\
(11.95)\end{array}$ & $\begin{array}{l}22.37 \\
(9.55)\end{array}$ & $\begin{array}{l}19.27- \\
25.47\end{array}$ & $\begin{array}{l}21.90 \\
(9.30)\end{array}$ & $\begin{array}{c}21.80 \\
(12.89)\end{array}$ & $\begin{array}{l}17.62- \\
25.98\end{array}$ & $\begin{array}{l}21.00 \\
(11.30)\end{array}$ \\
\hline $\begin{array}{l}\text { REM latency } \\
(\min )\end{array}$ & $\begin{array}{l}132.79 \\
(76.99)\end{array}$ & $\begin{array}{l}111.12- \\
154.22\end{array}$ & $\begin{array}{c}102.50 \\
(117.75)\end{array}$ & $\begin{array}{c}93.61 \\
(41.60)\end{array}$ & $\begin{array}{l}81.14- \\
107.26\end{array}$ & $\begin{array}{c}80.50 \\
(52.50)\end{array}$ & $\begin{array}{c}85.11 \\
(39.72)\end{array}$ & $\begin{array}{l}72.05- \\
98.26\end{array}$ & $\begin{array}{c}76.25 \\
(46.25)\end{array}$ \\
\hline $\begin{array}{l}\text { number of } \\
\text { awakenings }\end{array}$ & $\begin{array}{c}20.27 \\
(10.81)\end{array}$ & $\begin{array}{l}17.03- \\
23.72\end{array}$ & $\begin{array}{c}16.00 \\
(11.50)\end{array}$ & $\begin{array}{l}13.87 \\
(7.5)\end{array}$ & $\begin{array}{l}11.60- \\
16.21\end{array}$ & $\begin{array}{c}12.00 \\
(10.50)\end{array}$ & $\begin{array}{l}12.48 \\
(7.46)\end{array}$ & $9.92-14.97$ & $\begin{array}{c}11.00 \\
(11.50)\end{array}$ \\
\hline AI & $\begin{array}{c}17.41 \\
(10.16)\end{array}$ & $\begin{array}{l}14.08- \\
20.87\end{array}$ & $\begin{array}{c}14.10 \\
(17.10)\end{array}$ & $\begin{array}{c}14.73 \\
(9.46)\end{array}$ & $\begin{array}{c}12.01- \\
18.00\end{array}$ & $\begin{array}{c}12.30 \\
(18.00)\end{array}$ & $\begin{array}{l}11.59 \\
(8.37)\end{array}$ & $9.29-14.48$ & $\begin{array}{c}9.70 \\
(7.50)\end{array}$ \\
\hline
\end{tabular}




\section{References}

AASM. (2014). International Classification of Sleep Disorders: Diagnostic and Coding Manual (3rd edition ed.). Darien American Academy of Sleep Medicine

AASM. (2017). Standards and Guidelines for the Practice of Sleep Medicine Evaluation and Management of Chronic Insomnia in Adults: American Academy of Sleep Medicine.

AASM Manual for the Scoring Sleep and Associated Events. (2016). (Version 2.3 ed.): American Academy of Sleep Medicine

Abdel-Kader, K., Jhamb, M., Mandich, L. A., Yabes, J., Keene, R. M., Beach, S., . . Unruh, M. L. (2014). Ecological momentary assessment of fatigue, sleepiness, and exhaustion in ESKD. BMC Nephrology, 15, 29. doi:10.1186/1471-2369-15-29

Akerstedt, T., Hume, K., Minors, D., \& Waterhouse, J. (1997). Good sleep--its timing and physiological sleep characteristics. Journal of Sleep Research, 6(4), 221-229.

American Psychiatric Association (2013) Diagnostic and Statistical Manual of Mental Disorders ( $5^{\text {th }}$ ed). Arlignton, VA. American Psychiatric Publishing

Antony, M. M., Bieling, P. J., Cox, B. J., Enns, M. W., \& Swinson, R. P. (1998). Psychometric properties of the 42 -item and 21-item versions of the Depression Anxiety Stress Scales in clinical groups and a community sample. Psychological Assessment, 10, 176-181.

Atkinson, G., \& Reilly, T. (1996). Circadian variation in sports performance. Sports Medicine, 21(4), 292-312.

Auger, R. R., Burgess, H. J., Emens, J. S., Deriy, L. V., Thomas, S. M., \& Sharkey, K. M. (2015). Clinical Practice Guideline for the Treatment of Intrinsic Circadian Rhythm Sleep-Wake Disorders: Advanced Sleep-Wake Phase Disorder (ASWPD), Delayed Sleep-Wake Phase Disorder (DSWPD), Non-24-Hour Sleep-Wake Rhythm Disorder 
(N24SWD), and Irregular Sleep-Wake Rhythm Disorder (ISWRD). An Update for 2015: An American Academy of Sleep Medicine Clinical Practice Guideline. Journal of Clinical Sleep Medicine, 11(10), 1199-1236. doi:10.5664/jcsm.5100

Ayalon, L., Borodkin, K., Dishon, L., Kanety, H., \& Dagan, Y. (2007). Circadian rhythm sleep disorders following mild traumatic brain injury. Neurology, 68(14), 1136-1140. doi:10.1212/01.wnl.0000258672.52836.30

Baglioni, C., Regen, W., Teghen, A., Spiegelhalder, K., Feige, B., Nissen, C., \& Riemann, D. (2013). Sleep changes in the disorder of insomnia: A meta-analysis of polysomnographic studies. Sleep Medicine Reviews, 18, 195-213.

Banhiran, W., Chotinaiwattarakul, W., Chongkolwatana, C., \& Metheetrairut, C. (2014). Homebased diagnosis of obstructive sleep apnea by polysomnography type 2: accuracy, reliability, and feasibility. Sleep Breath, 18(4), 817-823. doi:10.1007/s11325-014-0949-1

Bastien, C. H., Vallieres, A., \& Morin, C. M. (2001). Validation of the Insomnia Severity Index as an outcome measure for insomnia research. Sleep Medicine, 2, 297-307.

Baumann, C. R., Werth, E., Stocker, R., Ludwig, S., \& Bassetti, C. L. (2007). Sleep-wake disturbances 6 months after traumatic brain injury: a prospective study. Brain, 130( $\mathrm{Pt} 7)$, 1873-1883. doi:10.1093/brain/awm109

Benloucif, S., Burgess, H. J., Klerman, E. B., Lewy, A. J., Middleton, B., Murphy, P. J., . . Revell, V. L. (2008). Measuring melatonin in humans. Journal of Clinical Sleep Medicine, 4(1), 66-69.

Bioulac, S., Franchi, J. M., Arnaud, M., Sagaspe, P., Moore, N., Salvo, F., \& Philip, P. (2017). Risk of Motor Vehicle Accidents Related to Sleepiness at the Wheel: A Systematic Review and Meta-Analysis. Sleep, 40(10). doi:10.1093/sleep/zsx 134 
Bjornsdottir, E., Janson, C., Sigurdsson, J. F., Gehrman, P., Perlis, M., Juliusson, S., . . .

Benediktsdottir, B. (2013). Symptoms of insomnia among patients with obstructive sleep apnea before and after two years of positive airway pressure treatment. Sleep, 36(12), 1901-1909. doi:10.5665/sleep.3226

Bjurstrom, M. F., \& Irwin, M. R. (2016). Polysomnographic characteristics in nonmalignant chronic pain populations: A review of controlled studies. Sleep Medicine Reviews, 26, 74-86. doi:10.1016/j.smrv.2015.03.004

Bohra, M. H., Kaushik, C., Temple, D., Chung, S. A., \& Shapiro, C. M. (2014). Weighing the balance: how analgesics used in chronic pain influence sleep? British Journal of Pain, 8(3), 107-118. doi:10.1177/2049463714525355

Broomfield, N. M., \& Espie, C. A. (2005). Towards a valid, reliable measure of sleep effort. $J$ Sleep Research, 14(4), 401-407. doi:10.1111/j.1365-2869.2005.00481.x

Bruyneel, M., Libert, W., Ameye, L., \& Ninane, V. (2015). Comparison between home and hospital set-up for unattended home-based polysomnography: a prospective randomized study. Sleep Medicine, 16(11), 1434-1438. doi:10.1016/j.sleep.2015.04.006

Budhiraja, R., Roth, T., Hudgel, D. W., Budhiraja, P., \& Drake, C. L. (2011). Prevalence and polysomnographic correlates of insomnia comorbid with medical disorders. Sleep, 34(7), 859-867.

Burgess, H. J., \& Eastman, C. I. (2005). The dim light melatonin onset following fixed and free sleep schedules. Journal of Sleep Research, 14(3), 229-237. doi:10.1111/j.13652869.2005.00470.x 
Burgess, H. J., \& Eastman, C. I. (2006). A late wake time phase delays the human dim light melatonin rhythm. Neuroscience Letters, 395(3), 191-195. doi:10.1016/j.neulet.2005.10.082

Burgess, H. J., \& Emens, J. S. (2016). Circadian-Based Therapies for Circadian Rhythm SleepWake Disorders. Current Sleep Medicine Reports, 2(3), 158-165. doi:10.1007/s40675016-0052-1

Burgess, H. J., Park, M., Wyatt, J. K., \& Fogg, L. F. (2016). Home dim light melatonin onsets with measures of compliance in delayed sleep phase disorder. Journal of Sleep Research, 25(3), 314-317. doi:10.1111/jsr.12384

Burgess, H. J., Revell, V. L., \& Eastman, C. I. (2008). A three pulse phase response curve to three milligrams of melatonin in humans. Journal of Physiology, 586(2), 639-647. doi:10.1113/jphysiol.2007.143180

Burgess, H. J., Wyatt, J. K., Park, M., \& Fogg, L. F. (2015). Home Circadian Phase Assessments with Measures of Compliance Yield Accurate Dim Light Melatonin Onsets. Sleep, 38(6), 889-897. doi:10.5665/sleep.4734

Buysse, D. J., Ancoli-Israel, S., Edinger, J. D., Lichstein, K. L., \& Morin, C. M. (2006). Recommendations for a standard research assessment of insomnia. Sleep, 29(9), 11551173.

Buysse, D. J., Cheng, Y., Germain, A., Moul, D. E., Franzen, P. L., Fletcher, M., \& Monk, T. H. (2010). Night-to-night sleep variability in older adults with and without chronic insomnia. Sleep Medicine, 11(1), 56-64. doi:10.1016/j.sleep.2009.02.010 
Buysse, D. J., Reynolds, C. F., 3rd, Monk, T. H., Berman, S. R., \& Kupfer, D. J. (1989). The Pittsburgh Sleep Quality Index: a new instrument for psychiatric practice and research. Psychiatry Research, 28(2), 193-213.

Cameron, L. D., \& Moss-Morris, R. (2004). Ilness-Related Cognition and Behavior. In E. Kaptein \& J. Weinman (Eds.), Health Psychology. Oxford, UK: Blackwell Publishing.

Campbell, C. M., Buenaver, L. F., Finan, P., Bounds, S. C., Redding, M., McCauley, L., ... Smith, M. T. (2015). Sleep, Pain Catastrophizing, and Central Sensitization in Knee Osteoarthritis Patients With and Without Insomnia. Arthritis Care and Research (Hoboken), 67(10), 1387-1396. doi:10.1002/acr.22609

Carney, Harris, Falco, \& Edinger, J. D. (2013). The relation between insomnia symptoms, mood, and rumination about insomnia symptoms. Journal of Clinical Sleep Medicine, 9(6), 567575. doi: $10.5664 /$ jcsm. 2752

Carney, C. E., Buysse, D. J., Ancoli-Israel, S., Edinger, J. D., Krystal, A. D., Lichstein, K. L., \& Morin, C. M. (2012). The consensus sleep diary: standardizing prospective sleep selfmonitoring. Sleep, 35(2), 287-302. doi:10.5665/sleep.1642

Carney, C. E., Edinger, J. D., Olsen, M. K., Stechuchak, K. M., Krystal, A., \& Wyatt, J. K. (2008). Inter-rater reliability for insomnia diagnoses derived from the Duke Structured Interview for Sleep Disorders. Sleep, 31 (Suppl.), A250.

Carney, C. E., Harris, A. L., Falco, A., \& Edinger, J. D. (2013). The Relation between Insomnia Symptoms, Mood and Rumination about Insomnia Symptoms. Journal of Clinical Sleep Medicine, 9, 567-575.

Cassidy, J. D., Carroll, L. J., Peloso, P. M., Borg, J., von Holst, H., Holm, L., . . Injury, W. H. O. C. C. T. F. o. M. T. B. (2004). Incidence, risk factors and prevention of mild traumatic 
brain injury: results of the WHO Collaborating Centre Task Force on Mild Traumatic Brain Injury. Journal of Rehabilitation Medicine (43 Suppl), 28-60.

Castriotta, R. J., Wilde, M. C., Lai, J. M., Atanasov, S., Masel, B. E., \& Kuna, S. T. (2007). Prevalence and consequences of sleep disorders in traumatic brain injury. Journal of Clinical Sleep Medicine, 3(4), 349-356.

Chow, H. M., Horovitz, S. G., Carr, W. S., Picchioni, D., Coddington, N., Fukunaga, M., .. . Braun, A. R. (2013). Rhythmic alternating patterns of brain activity distinguish rapid eye movement sleep from other states of consciousness. Proceedings of the National Academy of Sciences of the U S A, 110(25), 10300-10305. doi:10.1073/pnas.1217691110

Chung, F., Subramanyam, R., Liao, P., Sasaki, E., Shapiro, C., \& Sun, Y. (2012). High STOPBang score indicates a high probability of obstructive sleep apnoea. British Journal of Anaesthesia, 108(5), 768-775.

Chung, F., Yegneswaran, B., Liao, P., Chung, S. A., Vairavanathan, S., Islam, S., . . Shapiro, C. M. (2008). STOP questionnaire: a tool to screen patients for obstructive sleep apnea. Anesthesiology, 108(5), 812-821. doi:10.1097/ALN.0b013e31816d83e4

Coates, T. J., Killen, J. D., George, J., Marchini, E., Silverman, S., \& Thoresen, C. (1982). Estimating sleep parameters: a multitrait--multimethod analysis. Journal of Consulting and Clinical Psychology, 50(3), 345-352.

Collen, J., Orr, N., Lettieri, C. J., Carter, K., \& Holley, A. B. (2012). Sleep disturbances among soldiers with combat-related traumatic brain injury. Chest, 142(3), 622-630. doi:10.1378/chest.11-1603

Cronlein, T., Geisler, P., Langguth, B., Eichhammer, P., Jara, C., Pieh, C., . . Hajak, G. (2012a). Polysomnography reveals unexpectedly high rates of organic sleep disorders in patients 
with prediagnosed primary insomnia. Sleep Breath, 16(4), 1097-1103. doi:10.1007/s11325-011-0608-8

Cronlein, T., Geisler, P., Langguth, B., Eichhammer, P., Jara, C., Pieh, C., . . Hajak, G. (2012b). Polysomnography reveals unexpectedly high rates of organic sleep disorders in patients with prediagnosed primary insomnia. Sleep and Breathing, 16(4), 1097-1103. doi:10.1007/s11325-011-0608-8

Cronlein, T., Wagner, S., Langguth, B., Geisler, P., Eichhammer, P., \& Wetter, T. C. (2014). Are dysfunctional attitudes and beliefs about sleep unique to primary insomnia? Sleep Medicine, 15(12), 1463-1467. doi:10.1016/j.sleep.2014.06.018

Curran, S. L., Beacham, A. O., \& Andrykowski, M. A. (2004). Ecological momentary assessment of fatigue following breast cancer treatment. Journal of Behavioral Medicine, $27(5), 425-444$.

Dewald, J. F., Meijer, A. M., Oort, F. J., Kerkhof, G. A., \& Bogels, S. M. (2010). The influence of sleep quality, sleep duration and sleepiness on school performance in children and adolescents: A meta-analytic review. Sleep Medicine Reviews, 14(3), 179-189. doi:10.1016/j.smrv.2009.10.004

Diagnostic and Statistical Manual of Mental Disorders, (DSM-5 ). (2013). Washington, DC: American Psychiatric Pub.

Dijk, D. J. (2009). Regulation and functional correlates of slow wave sleep. Journal of Clinical Sleep Medicine, 5(2 Suppl), S6-15.

Dittner, A. J., Wessely, S. C., \& Brown, R. G. (2004). The assessment of fatigue: a practical guide for clinicians and researchers. Journal of Psychosomatic Research, 56(2), 157-170. 
Douglass, A. B., Bornstein, R., Nino-Murcia, G., Keenan, S., Miles, L., Zarcone, V. P., Jr., . . Dement, W. C. (1994). The Sleep Disorders Questionnaire. I: Creation and multivariate structure of SDQ. Sleep, 17(2), 160-167.

Drake, Hays, R. D., Morlock, R., Wang, F., Shikiar, R., Frank, L., . . Roth, T. (2014).

Development and evaluation of a measure to assess restorative sleep. Journal of Clinical Sleep Medicine, 10(7), 733-741, 741A-741E. doi:10.5664/jcsm.3860

Drake, C. L., Roehrs, T., Breslau, N., Johnson, E., Jefferson, C., Scofield, H., \& Roth, T. (2010). The 10-year risk of verified motor vehicle crashes in relation to physiologic sleepiness. Sleep, 33(6), 745-752.

Edinger, J. D., Fins, A. I., Sullivan, R. J., Jr., Marsh, G. R., Dailey, D. S., Hope, T. V., . . . Vasilas, D. (1997). Sleep in the laboratory and sleep at home: comparisons of older insomniacs and normal sleepers. Sleep, 20(12), 1119-1126.

Edinger, J. D., Hoelscher, T. J., Webb, M. D., Marsh, G. R., Radtke, R. A., \& Erwin, C. W. (1989). Polysomnographic assessment of DIMS: empirical evaluation of its diagnostic value. Sleep, 12(4), 315-322.

Edinger, J. D., Kirby, A., Lineberger, M., Loiselle, M., Wohlgemuth, W., \& Means, M. (2004). The Duke Structured Interview for Sleep Disorders. In D. U. M. Center (Ed.).

Edinger, J. D., Wohlgemuth, W., Wyatt, J. K., \& Krystal, A. (2015). SSM-IV versus DSM 5: Are we going in the right direction with our insomnia classification efforts? . Paper presented at the SLEEP 2015, Seattle, WA.

Fairholme, C. P., \& Manber, R. (2014). Safety behaviors and sleep effort predict sleep disturbance and fatigue in an outpatient sample with anxiety and depressive disorders. 
Journal of Psychosomatic Research, 76(3), 233-236.

doi:10.1016/j.jpsychores.2014.01.001

Feige, B., Al-Shajlawi, A., Nissen, C., Voderholzer, U., Hornyak, M., Spiegelhalder, K., . . . Riemann, D. (2008). Does REM sleep contribute to subjective wake time in primary insomnia? A comparison of polysomnographic and subjective sleep in 100 patients. $J$ Sleep Research, 17(2), 180-190. doi:10.1111/j.1365-2869.2008.00651.x

Feige, B., Baglioni, C., Spiegelhalder, K., Hirscher, V., Nissen, C., \& Riemann, D. (2013). The microstructure of sleep in primary insomnia: an overview and extension. International Journal of Psychophysiology, 89(2), 171-180. doi:10.1016/j.ijpsycho.2013.04.002

Finan, P. H., Goodin, B. R., \& Smith, M. T. (2013). The association of sleep and pain: an update and a path forward. Journal of Pain, 14(12), 1539-1552. doi:10.1016/j.jpain.2013.08.007

Geiger-Brown, J. M., Rogers, V. E., Liu, W., Ludeman, E. M., Downton, K. D., \& Diaz-Abad, M. (2015). Cognitive behavioral therapy in persons with comorbid insomnia: A metaanalysis. Sleep Medicine Reviews, 23, 54-67. doi:10.1016/j.smrv.2014.11.007

Glidewell, R. N., Renn, B. N., Roby, E., \& Orr, W. C. (2014). Predictors and patterns of insomnia symptoms in OSA before and after PAP therapy. Sleep Medicine, 15(8), 899905. doi:10.1016/j.sleep.2014.05.001

Goril, S., Zalai, D., Scott, L., \& Shapiro, C. M. (2016). Sleep and melatonin secretion abnormalities in children and adolescents with fetal alcohol spectrum disorders. Sleep Medicine, 23, 59-64. doi:10.1016/j.sleep.2016.06.002

Grima, N. A., Ponsford, J., Rajaratnam, S. M., Mansfield, D., \& Pase, M. P. (2016). Sleep Disturbances in Traumatic Brain Injury: A Meta-Analysis. Journal of Clinical Sleep Medicine, 12(3), 419-428. doi:10.5664/jcsm.5598 
Grima, N. A., Ponsford, J. L., St Hilaire, M. A., Mansfield, D., \& Rajaratnam, S. M. (2016). Circadian Melatonin Rhythm Following Traumatic Brain Injury. Neurorehabilitation and Neural Repair, 30(10), 972-977. doi:10.1177/1545968316650279

Guaraldi, P., Sancisi, E., La Morgia, C., Calandra-Buonaura, G., Carelli, V., Cameli, O., .. . Piperno, R. (2014). Nocturnal melatonin regulation in post-traumatic vegetative state: a possible role for melatonin supplementation? Chronobiolology International, 31(5), 741745. doi:10.3109/07420528.2014.901972

Guilleminault, C., Yuen, K.M., Gulevich, M.G., Karadeniz, D., Leger, D \& Philip, P. (2000). Hypersomnia after head-neck trauma: a medicolegal dilemma. Neurology, 54(3), 653659.

Hacker, E. D., \& Ferrans, C. E. (2007). Ecological momentary assessment of fatigue in patients receiving intensive cancer therapy. Journal of Pain and Symptom Management, 33(3), 267-275. doi:10.1016/j.jpainsymman.2006.08.007

Halasz, P., Terzano, M., Parrino, L., \& Bodizs, R. (2004). The nature of arousal in sleep. Journal of Sleep Research, 13(1), 1-23.

Harris, A., Zalai, D., Moss, T. G., \& Carney, C. E. (2016). The relationship between sleep and fatigue in chronic insomnia: the role of sleep perception, beliefs and attitudes about sleep, and fatigue-specific cognitions. Journal of Psychosomatic Research (submitted for publication).

Hartmann, J. A., Carney, C. E., Lachowski, A., \& Edinger, J. D. (2015). Exploring the construct of subjective sleep quality in patients with insomnia. Journal of Clinical Psychiatry, 76(6), e768-773. doi:10.4088/JCP.14m09066 
Harvey, A. G., Sharpley, A. L., Ree, M. J., Stinson, K., \& Clark, D. M. (2007). An open trial of cognitive therapy for chronic insomnia. Behavior Research and Therapy, 45(10), 24912501. doi:10.1016/j.brat.2007.04.007

Henry, J. D., \& Crawford, J. R. (2005). The short-form version of the Depression Anxiety Stress Scales (DASS-21): construct validity and normative data in a large non-clinical sample. The British Journal of Clinical Psychology, 44(Pt 2), 227-239.

Hertenstein, E., Nissen, C., Riemann, D., Feige, B., Baglioni, C., \& Spiegelhalder, K. (2015). The exploratory power of sleep effort, dysfunctional beliefs and arousal for insomnia severity and polysomnography-determined sleep. Journal of Sleep Research, 24(4), 399406. doi:10.1111/jsr.12293

Hirscher, V., Unbehaun, T., Feige, B., Nissen, C., Riemann, D., \& Spiegelhalder, K. (2015). Patients with primary insomnia in the sleep laboratory: do they present with typical nights of sleep? Journal of Sleep Research, 24(4), 383-389. doi:10.1111/jsr.12280

Hjermstad, M. J., Fayers, P. M., Haugen, D. F., Caraceni, A., Hanks, G. W., Loge, J. H., . . European Palliative Care Research, C. (2011). Studies comparing Numerical Rating Scales, Verbal Rating Scales, and Visual Analogue Scales for assessment of pain intensity in adults: a systematic literature review. Journal of Pain and Symptom Management, 41(6), 1073-1093. doi:10.1016/j.jpainsymman.2010.08.016

Horne, J. A., \& Ostberg, O. (1976). A self-assessment questionnaire to determine morningnesseveningness in human circadian rhythms. International Journal of Chronobiology, 4(2), $97-110$. 
Insana, S. P., Gozal, D., \& Montgomery-Downs, H. E. (2010). Invalidity of one actigraphy brand for identifying sleep and wake among infants. Sleep Medicine, 11(2), 191-196. doi:10.1016/j.sleep.2009.08.010

International Classification of Sleep Disorders: Diagnostic and Coding Manual. (2014). (Third ed.): American Academy of Sleep Medicine

Jacobs, E. A., Reynolds, C. F., 3rd, Kupfer, D. J., Lovin, P. A., \& Ehrenpreis, A. B. (1988). The role of polysomnography in the differential diagnosis of chronic insomnia. American Journal of Psychiatry, 145(3), 346-349. doi:10.1176/ajp.145.3.346

Jansson-Frojmark, M., Harvey, A. G., Lundh, L. G., Norell-Clarke, A., \& Linton, S. J. (2011). Psychometric properties of an insomnia-specific measure of worry: the Anxiety and Preoccupation about Sleep Questionnaire. Cognitive Behavioral Therapy, 40(1), 65-76. doi:10.1080/16506073.2010.538432

Kantermann, T., Sung, H., \& Burgess, H. J. (2015). Comparing the Morningness-Eveningness Questionnaire and Munich Chronotype Questionnaire to dim light melatonin onset. Journal of Biologcal Rhythms, 30(5), 449-453.

Keijzer, H., Smits, M. G., Duffy, J. F., \& Curfs, L. M. (2014). Why the dim light melatonin onset (DLMO) should be measured before treatment of patients with circadian rhythm sleep disorders. Sleep Medicine Reviews, 18(4), 333-339. doi:10.1016/j.smrv.2013.12.001

Kim, J., Kikuchi, H., \& Yamamoto, Y. (2013). Systematic comparison between ecological momentary assessment and day reconstruction method for fatigue and mood states in healthy adults. British Journal of Health Psychology, 18(1), 155-167. doi:10.1111/bjhp.12000 
Kinugawa, K., Doulazmi, M., Sebban, C., Schumm, S., Mariani, J., \& Nguyen-Michel, V. H. (2012). Sleep apnea in elderly adults with chronic insomnia. Journal of the American Geriatric Society, 60(12), 2366-2368. doi:10.1111/jgs.12006

Koffel, E. A., Koffel, J. B., \& Gehrman, P. R. (2015). A meta-analysis of group cognitive behavioral therapy for insomnia. Sleep Medicine Reviews, 19, 6-16. doi:10.1016/j.smrv.2014.05.001

Krakow, B. J., Ulibarri, V. A., Moore, B. A., \& McIver, N. D. (2015). Posttraumatic stress disorder and sleep-disordered breathing: a review of comorbidity research. Sleep Medicine Reviews, 24, 37-45. doi:10.1016/j.smrv.2014.11.001

Kratz, A. L., Murphy, S. L., \& Braley, T. J. (2017). Ecological Momentary Assessment of Pain, Fatigue, Depressive, and Cognitive Symptoms Reveals Significant Daily Variability in Multiple Sclerosis. Archives of Physical Medicine and Rehabilitation, 98(11), 2142-2150. doi:10.1016/j.apmr.2017.07.002

Krupp, L. B., LaRocca, N. G., Muir-Nash, J., \& Steinberg, A. D. (1989). The fatigue severity scale. Application to patients with multiple sclerosis and systemic lupus erythematosus. Archives of Neurology, 46(10), 1121-1123.

Lerman, S. F., Finan, P. H., Smith, M. T., \& Haythornthwaite, J. A. (2017). Psychological interventions that target sleep reduce pain catastrophizing in knee osteoarthritis. Pain, 158(11), 2189-2195. doi:10.1097/j.pain.0000000000001023

Lewy, A. J., Ahmed, S., Jackson, J. M., \& Sack, R. L. (1992). Melatonin shifts human circadian rhythms according to a phase-response curve. Chronobiolology International, 9(5), 380392. 
Lewy, A. J., Emens, J. S., Sack, R. L., Hasler, B. P., \& Bernert, R. A. (2002). Low, but not high, doses of melatonin entrained a free-running blind person with a long circadian period. Chronobiology International, 19(3), 649-658.

Lichstein, K. L., Riedel, B. W., Lester, K. W., \& Aguillard, R. N. (1999). Occult sleep apnea in a recruited sample of older adults with insomnia. Journal of Consulting and Clinical Psychololgy, 67(3), 405-410.

Lichstein, K. L., Stone, K. C., Donaldson, J., Nau, S. D., Soeffing, J. P., Murray, D., .. . Aguillard, R. N. (2006). Actigraphy validation with insomnia. Sleep, 29(2), 232-239.

Littner, M., Hirshkowitz, M., Kramer, M., Kapen, S., Anderson, W. M., Bailey, D., .. . Woodson, B. T. (2003). Practice parameters for using polysomnography to evaluate insomnia: an update. Sleep, 26(6), 754-760.

Lovato, N., Gradisar, M., Short, M., Dohnt, H., \& Micic, G. (2013). Delayed sleep phase disorder in an Australian school-based sample of adolescents. Journal of Clinical Sleep Medicine, 9(9), 939-944. doi:10.5664/jcsm.2998

Lovato, N., Micic, G., Gradisar, M., Ferguson, S. A., Burgess, H. J., Kennaway, D. J., \& Lack, L. (2016). Can the circadian phase be estimated from self-reported sleep timing in patients with Delayed Sleep Wake Phase Disorder to guide timing of chronobiologic treatment? Chronobiology International, 33(10), 1376-1390.

doi:10.1080/07420528.2016.1220386

Lovibond, P. F., \& Lovibond, S. H. (1995). The structure of negative emotional states: comparison of the Depression Anxiety Stress Scales (DASS) with the Beck Depression and Anxiety Inventories. Behavioral Research and Therapy, 33(3), 335-343. 
Maes, J., Verbraecken, J., Willemen, M., De Volder, I., van Gastel, A., Michiels, N., . . Cluydts, R. (2014). Sleep misperception, EEG characteristics and autonomic nervous system activity in primary insomnia: a retrospective study on polysomnographic data. International Journal of Psychophysiology, 91(3), 163-171. doi:10.1016/j.ijpsycho.2013.10.012

Maich, K. H., Lachowski, A. M., \& Carney, C. E. (2016). Psychometric Properties of the Consensus Sleep Diary in Those With Insomnia Disorder. Behavioral Sleep Medicine, 118. doi:10.1080/15402002.2016.1173556

Marino, M., Li, Y., Rueschman, M. N., Winkelman, J. W., Ellenbogen, J. M., Solet, J. M., . . Buxton, O. M. (2013). Measuring sleep: accuracy, sensitivity, and specificity of wrist actigraphy compared to polysomnography. Sleep, 36(11), 1747-1755. doi:10.5665/sleep.3142

Martinez, M. P., Miro, E., Sanchez, A. I., Diaz-Piedra, C., Caliz, R., Vlaeyen, J. W., \& BuelaCasal, G. (2014). Cognitive-behavioral therapy for insomnia and sleep hygiene in fibromyalgia: a randomized controlled trial. Journal of Behavioral Medicine, 37(4), 683697. doi:10.1007/s10865-013-9520-y

Mathias, J. L., \& Alvaro, P. K. (2012). Prevalence of sleep disturbances, disorders, and problems following traumatic brain injury: a meta-analysis. Sleep Medicine, 13(7), 898-905. doi:10.1016/j.sleep.2012.04.006

Means, M. K., Edinger, J. D., Glenn, D. M., \& Fins, A. I. (2003a). Accuracy of sleep perceptions among insomnia sufferers and normal sleepers. Sleep Medicine, 4(4), 285-296.

Means, M. K., Edinger, J. D., Glenn, D. M., \& Fins, A. I. (2003b). Accuracy of sleep perceptions among insomnia sufferers and normal sleepers. Sleep Medicine, 4(4), 285-296. 
Melamed, S., \& Oksenberg, A. (2002). Excessive daytime sleepiness and risk of occupational injuries in non-shift daytime workers. Sleep, 25(3), 315-322.

Micic, G., Lovato, N., Gradisar, M., Ferguson, S. A., Burgess, H. J., \& Lack, L. C. (2016). The etiology of delayed sleep phase disorder. Sleep Medicine Reviews, 27, 29-38. doi:10.1016/j.smrv.2015.06.004

Milewski, M. D., Skaggs, D. L., Bishop, G. A., Pace, J. L., Ibrahim, D. A., Wren, T. A., \& Barzdukas, A. (2014). Chronic lack of sleep is associated with increased sports injuries in adolescent athletes. Journal of Pediatric Orthopedics, 34(2), 129-133. doi:10.1097/BPO.0000000000000151

Modarres, M., Kuzma, N. N., Kretzmer, T., Pack, A. I., \& Lim, M. M. (2016). EEG slow waves in traumatic brain injury: Convergent findings in mouse and man. Neurobiology of Sleep Circadian Rhythms, 1.

Mollayeva, T., Shapiro, C. M., Mollayeva, S., Cassidy, J. D., \& Colantonio, A. (2015). Modeling community integration in workers with delayed recovery from mild traumatic brain injury. BMC Neurology, 15, 194. doi:10.1186/s12883-015-0432-z

Morgenthaler, T. I., Lee-Chiong, T., Alessi, C., Friedman, L., Aurora, R. N., Boehlecke, B., . . . Standards of Practice Committee of the American Academy of Sleep, M. (2007). Practice parameters for the clinical evaluation and treatment of circadian rhythm sleep disorders. An American Academy of Sleep Medicine report. Sleep, 30(11), 1445-1459.

Morin, C. M. (1993a). Insomnia: Psychological assessment and management. New York, NY: Guilford Press.

Morin, C. M. (1993b). Insomnia: Psychological Assessment and Management. New York: The Guilford Press. 
Morin, C. M. (1994). Dysfunctional beliefs and attitudes about sleep: Preliminary scale development and description. . The Behavior Therapist, Summer, 163-164.

Morin, C. M., Belleville, G., Belanger, L., \& Ivers, H. (2011). The Insomnia Severity Index: psychometric indicators to detect insomnia cases and evaluate treatment response. Sleep, 34(5), 601-608.

Morin, C. M., Culbert, J. P., \& Schwartz, S. M. (1994). Nonpharmacological interventions for insomnia: a meta-analysis of treatment efficacy. American Journal of Psychiatry, 151(8), 1172-1180. doi:10.1176/ajp.151.8.1172

Morin, C. M., Vallieres, A., \& Ivers, H. (2007). Dysfunctional beliefs and attitudes about sleep (DBAS): validation of a brief version (DBAS-16). Sleep, 30(11), 1547-1554.

Nagappa, M., Liao, P., Wong, J., Auckley, D., Ramachandran, S. K., Memtsoudis, S., . . Chung, F. (2015). Validation of the STOP-Bang Questionnaire as a Screening Tool for Obstructive Sleep Apnea among Different Populations: A Systematic Review and MetaAnalysis. PLoS One, 10(12), e0143697. doi:10.1371/journal.pone.0143697

Natale, V., Plazzi, G., \& Martoni, M. (2009). Actigraphy in the assessment of insomnia: a quantitative approach. Sleep, 32(6), 767-771.

Normand, M. P., St-Hilaire, P., \& Bastien, C. H. (2016). Sleep Spindles Characteristics in Insomnia Sufferers and Their Relationship with Sleep Misperception. Neural Plasticity, 2016, 6413473. doi:10.1155/2016/6413473

Ohayon, M. M., \& Roth, T. (2003). Place of chronic insomnia in the course of depressive and anxiety disorders. Journal of Psychiatric Research, 37(1), 9-15.

ONF. (2013). Guidelines for Concussion/Traumatic Brain Injury \& Persistent Symptoms. In O. N. Foundation (Ed.), (Second ed.). Toronto, Canada. 
Ong, J. C., Gress, J. L., San Pedro-Salcedo, M. G., \& Manber, R. (2009). Frequency and predictors of obstructive sleep apnea among individuals with major depressive disorder and insomnia. Journal of Psychosomatic Research, 67(2), 135-141. doi:10.1016/j.jpsychores.2009.03.011

Osborn, A. J., Mathias, J. L., \& Fairweather-Schmidt, A. K. (2014). Depression following adult, non-penetrating traumatic brain injury: a meta-analysis examining methodological variables and sample characteristics. Neuroscience and Biobehavioral Reviews, 47, 1-15. doi:10.1016/j.neubiorev.2014.07.007

Ouellet, M. C., \& Morin, C. M. (2007). Efficacy of cognitive-behavioral therapy for insomnia associated with traumatic brain injury: a single-case experimental design. Archives of Physical Medicine and Rehabilitation, 88(12), 1581-1592.

doi:10.1016/j.apmr.2007.09.006

Paine, S. J., Fink, J., Gander, P. H., \& Warman, G. R. (2014). Identifying advanced and delayed sleep phase disorders in the general population: a national survey of New Zealand adults. Chronobiology International, 31(5), 627-636. doi:10.3109/07420528.2014.885036

Paine, S. J., Gander, P. H., \& Travier, N. (2006). The epidemiology of morningness/eveningness: influence of age, gender, ethnicity, and socioeconomic factors in adults (30-49 years). $J$ Biological Rhythms, 21(1), 68-76. doi:10.1177/0748730405283154

Paparrigopoulos, T., Melissaki, A., Tsekou, H., Efthymiou, A., Kribeni, G., Baziotis, N., \& Geronikola, X. (2006). Melatonin secretion after head injury: a pilot study. Brain Injury, 20(8), 873-878. doi:10.1080/02699050600832114

Paquet, J., Kawinska, A., \& Carrier, J. (2007). Wake detection capacity of actigraphy during sleep. Sleep, 30(10), 1362-1369. 
Parrino, L., Ferri, R., Bruni, O., \& Terzano, M. G. (2012). Cyclic alternating pattern (CAP): the marker of sleep instability. Sleep Medicine Reviews, 16(1), 27-45. doi:10.1016/j.smrv.2011.02.003

Parrino, L., Milioli, G., De Paolis, F., Grassi, A., \& Terzano, M. G. (2009). Paradoxical insomnia: the role of CAP and arousals in sleep misperception. Sleep Medicine, 10(10), 1139-1145. doi:10.1016/j.sleep.2008.12.014

Parrott, A. C., \& Hindmarch, I. (1978). Factor analysis of a sleep evaluation questionnaire. Psychological Medicine, 8(2), 325-329.

Paul, T., \& Lemmer, B. (2007). Disturbance of circadian rhythms in analgosedated intensive care unit patients with and without craniocerebral injury. Chronobiology International, 24(1), 45-61. doi:10.1080/07420520601142569

Perlis, M. L., Smith, M. T., Andrews, P. J., Orff, H., \& Giles, D. E. (2001). Beta/Gamma EEG activity in patients with primary and secondary insomnia and good sleeper controls. Sleep, 24(1), 110-117.

Pillai, V., Roth, T., \& Drake, C. L. (2015). The nature of stable insomnia phenotypes. Sleep, 38(1), 127-138. doi:10.5665/sleep.4338

Powell, D. J., Liossi, C., Schlotz, W., \& Moss-Morris, R. (2017). Tracking daily fatigue fluctuations in multiple sclerosis: ecological momentary assessment provides unique insights. Journal of Behavioral Medicine. doi:10.1007/s10865-017-9840-4

Quellet, M. C., \& Morin, C. M. (2006). Subjective and objective measures of insomnia in the context of traumatic brain injury: a preliminary study. Sleep Medicine, 7(6), 486-497. 
Rahman, S. A., Kayumov, L., Tchmoutina, E. A., \& Shapiro, C. M. (2009). Clinical efficacy of dim light melatonin onset testing in diagnosing delayed sleep phase syndrome. Sleep Medicine, 10(5), 549-555. doi:10.1016/j.sleep.2008.03.020

Rao, V., Bergey, A., Hill, H., Efron, D., \& McCann, U. (2011). Sleep disturbance after mild traumatic brain injury: indicator of injury? Journal of Neuropsychiatry and Clinical Neuroscience, 23(2), 201-205. doi:10.1176/appi.neuropsych.23.2.201

10.1176/jnp.23.2.jnp201

Riemann, D., Klein, T., Rodenbeck, A., Feige, B., Horny, A., Hummel, R., . . Voderholzer, U. (2002). Nocturnal cortisol and melatonin secretion in primary insomnia. Psychiatry Research, 113(1-2), 17-27.

Riemann, D., Spiegelhalder, K., Nissen, C., Hirscher, V., Baglioni, C., \& Feige, B. (2012). REM sleep instability--a new pathway for insomnia? Pharmacopsychiatry, 45(5), 167-176. doi:10.1055/s-0031-1299721

Rosenwasser, A. M. (2015). Chronobiology of ethanol: animal models. Alcohol, 49(4), 311-319. doi:10.1016/j.alcohol.2015.04.001

Ruff, R. M., Iverson, G. L., Barth, J. T., Bush, S. S., \& Broshek, D. K. (2009).

Recommendations for diagnosing a mild traumatic brain injury: a National Academy of Neuropsychology education paper. Archives of Clinical Neuropsychology, 24(1), 3-10. doi:10.1093/arclin/acp006

Sadeh, A. (2011). The role and validity of actigraphy in sleep medicine: an update. Sleep Medicine Reviews, 15(4), 259-267. doi:10.1016/j.smrv.2010.10.001 
Sanchez-Ortuno, M. M., Edinger, J. D., Means, M. K., \& Almirall, D. (2010a). Home is where sleep is: an ecological approach to test the validity of actigraphy for the assessment of insomnia. Journal of Clinical Sleep Medicine, 6(1), 21-29.

Sanchez-Ortuno, M. M., Edinger, J. D., Means, M. K., \& Almirall, D. (2010b). Home is where sleep is: an ecological approach to test the validity of actigraphy for the assessment of insomnia. Journal of Cinical Sleep Medicine, 6(1), 21-29.

Saxvig, I. W., Pallesen, S., Wilhelmsen-Langeland, A., Molde, H., \& Bjorvatn, B. (2012).

Prevalence and correlates of delayed sleep phase in high school students. Sleep Medicine, 13(2), 193-199. doi:10.1016/j.sleep.2011.10.024

Schrader, H., Bovim, G., \& Sand, T. (1993). The prevalence of delayed and advanced sleep phase syndromes. Journal of Sleep Research, 2(1), 51-55.

Schutte-Rodin, S., Broch, L., Buysse, D., Dorsey, C., \& Sateia, M. (2008). Clinical guideline for the evaluation and management of chronic insomnia in adults. Journal of Clinical Sleep Medicine, 4(5), 487-504.

Seifman, M. A., Adamides, A. A., Nguyen, P. N., Vallance, S. A., Cooper, D. J., Kossmann, T., . . Morganti-Kossmann, M. C. (2008). Endogenous melatonin increases in cerebrospinal fluid of patients after severe traumatic brain injury and correlates with oxidative stress and metabolic disarray. Journal of Cerebral Blood Flow and Metabolism, 28(4), 684696. doi:10.1038/sj.jcbfm.9600603

Series, F., Roy, N., \& Marc, I. (1994). Effects of sleep deprivation and sleep fragmentation on upper airway collapsibility in normal subjects. American Journal of Respiratory Critical Care Medicine, 150(2), 481-485. doi:10.1164/ajrccm.150.2.8049833 
Shapiro, C. M., Bortz, R., Mitchell, D., Bartel, P., \& Jooste, P. (1981). Slow-wave sleep: a recovery period after exercise. Science, 214(4526), 1253-1254.

Shekleton, J. A., Parcell, D. L., Redman, J. R., Phipps-Nelson, J., Ponsford, J. L., \& Rajaratnam, S. M. (2010). Sleep disturbance and melatonin levels following traumatic brain injury. Neurology, 74(21), 1732-1738. doi:10.1212/WNL.0b013e3181e0438b

Silva, E. J., Wang, W., Ronda, J. M., Wyatt, J. K., \& Duffy, J. F. (2010). Circadian and wakedependent influences on subjective sleepiness, cognitive throughput, and reaction time performance in older and young adults. Sleep, 33(4), 481-490.

Sinclair, K. L., Ponsford, J., \& Rajaratnam, S. M. (2014). Actigraphic assessment of sleep disturbances following traumatic brain injury. Behavioural Sleep Medicine, 12(1), 13-27. doi:10.1080/15402002.2012.726203

Sitnick, S. L., Goodlin-Jones, B. L., \& Anders, T. F. (2008). The use of actigraphy to study sleep disorders in preschoolers: some concerns about detection of nighttime awakenings. Sleep, $31(3), 395-401$.

Sivertsen, B., Omvik, S., Havik, O. E., Pallesen, S., Bjorvatn, B., Nielsen, G. H., . . Nordhus, I. H. (2006). A comparison of actigraphy and polysomnography in older adults treated for chronic primary insomnia. Sleep, 29(10), 1353-1358.

Sivertsen, B., Pallesen, S., Stormark, K. M., Boe, T., Lundervold, A. J., \& Hysing, M. (2013). Delayed sleep phase syndrome in adolescents: prevalence and correlates in a large population based study. BMC Public Health, 13, 1163. doi:10.1186/1471-2458-13-1163

Spielman, A. J., Caruso, L. S., \& Glovinsky, P. B. (1987). A behavioral perspective on insomnia treatment. Psychiatric Clinics of North America, 10(4), 541-553. 
Steele, D. L., Rajaratnam, S. M., Redman, J. R., \& Ponsford, J. L. (2005). The effect of traumatic brain injury on the timing of sleep. Chronobiol International, 22(1), 89-105.

Taillard, J., Philip, P., Chastang, J. F., \& Bioulac, B. (2004). Validation of Horne and Ostberg morningness-eveningness questionnaire in a middle-aged population of French workers. $J$ Biological Rhythms, 19(1), 76-86. doi:10.1177/0748730403259849

Tang, N. K., Goodchild, C. E., Sanborn, A. N., Howard, J., \& Salkovskis, P. M. (2012).

Deciphering the temporal link between pain and sleep in a heterogeneous chronic pain patient sample: a multilevel daily process study. Sleep, 35(5), 675-687A. doi:10.5665/sleep. 1830

Tang, N. K., \& Harvey, A. G. (2004). Correcting distorted perception of sleep in insomnia: a novel behavioural experiment? Behavior Research and Therapy, 42(1), 27-39.

Theadom, A., Cropley, M., Parmar, P., Barker-Collo, S., Starkey, N., Jones, K., . . Group, B. R. (2015). Sleep difficulties one year following mild traumatic brain injury in a populationbased study. Sleep Medicine, 16(8), 926-932. doi:10.1016/j.sleep.2015.04.013

Theadom, A., Starkey, N., Jones, K., Cropley, M., Parmar, P., Barker-Collo, S., \& Feigin, V. L. (2016). Sleep difficulties and their impact on recovery following mild traumatic brain injury in children. Brain Injury, 30(10), 1243-1248. doi:10.1080/02699052.2016.1183171

Tononi, G., \& Cirelli, C. (2014). Sleep and the price of plasticity: from synaptic and cellular homeostasis to memory consolidation and integration. Neuron, 81(1), 12-34. doi:10.1016/j.neuron.2013.12.025

Trotti, L. M., Bliwise, D. L., Greer, S. A., Sigurdsson, A. P., Gudmundsdottir, G. B., Wessel, T., . . Rye, D. B. (2009). Correlates of PLMs variability over multiple nights and impact upon RLS diagnosis. Sleep Medicine, 10(6), 668-671. doi:10.1016/j.sleep.2008.06.009 
Vallieres, A., \& Morin, C. M. (2003). Actigraphy in the assessment of insomnia. Sleep, 26(7), 902-906.

Van Veen, M. M., Kooij, J. J., Boonstra, A. M., Gordijn, M. C., \& Van Someren, E. J. (2010). Delayed circadian rhythm in adults with attention-deficit/hyperactivity disorder and chronic sleep-onset insomnia. Biological Psychiatry, 67(11), 1091-1096. doi:10.1016/j.biopsych.2009.12.032

van Wieringen, S., Jansen, T., Smits, M. G., Nagtegaal, J. E., \& Coenen, A. M. L. (2001). Melatonin for Chronic Whiplash Syndrome with Delayed Melatonin Onset. Clinical Drug Investigation, 21(12), 813-820.

Verma, A., Anand, V., \& Verma, N. P. (2007). Sleep disorders in chronic traumatic brain injury. Journal of Clinical Sleep Medicine, 3(4), 357-362.

Vernon, M. K., Dugar, A., Revicki, D., Treglia, M., \& Buysse, D. (2010). Measurement of nonrestorative sleep in insomnia: A review of the literature. Sleep Medicine Reviews, 14(3), 205-212. doi:10.1016/j.smrv.2009.10.002

Vitiello, M. V., McCurry, S. M., Shortreed, S. M., Baker, L. D., Rybarczyk, B. D., Keefe, F. J., \& Von Korff, M. (2014). Short-term improvement in insomnia symptoms predicts longterm improvements in sleep, pain, and fatigue in older adults with comorbid osteoarthritis and insomnia. Pain, 155(8), 1547-1554. doi:10.1016/j.pain.2014.04.032

Vitiello, M. V., McCurry, S. M., Shortreed, S. M., Balderson, B. H., Baker, L. D., Keefe, F. J., . . . Von Korff, M. (2013). Cognitive-behavioral treatment for comorbid insomnia and osteoarthritis pain in primary care: the lifestyles randomized controlled trial. Journal of the American Geriatric Society, 61(6), 947-956. doi:10.1111/jgs.12275 
Vitiello, M. V., Rybarczyk, B., Von Korff, M., \& Stepanski, E. J. (2009). Cognitive behavioral therapy for insomnia improves sleep and decreases pain in older adults with co-morbid insomnia and osteoarthritis. Journal of Clinical Sleep Medicine, 5(4), 355-362.

Weitzman, E. D., Czeisler, C. A., Coleman, R. M., Spielman, A. J., Zimmerman, J. C., Dement, W., . . Pollak, C. P. (1981). Delayed sleep phase syndrome. A chronobiological disorder with sleep-onset insomnia. Archives of General Psychiatry, 38(7), 737-746.

Wilkinson, K., \& Shapiro, C. (2013). Development and validation of the Nonrestorative Sleep Scale (NRSS). Journal of Clinical Sleep Medicine, 9(9), 929-937. doi:10.5664/jcsm.2996

Wohlgemuth, W. K., Edinger, J. D., Fins, A. I., \& Sullivan, R. J., Jr. (1999). How many nights are enough? The short-term stability of sleep parameters in elderly insomniacs and normal sleepers. Psychophysiology, 36(2), 233-244.

Wright, H., Lack, L., \& Bootzin, R. R. (2006). Relationship between dim light melatonin onset and the timing of sleep in sleep onset insmniacs Sleep and Biological Rhythms, 4, 78-80.

Yaeger, K., Alhilali, L., \& Fakhran, S. (2014). Evaluation of tentorial length and angle in sleepwake disturbances after mild traumatic brain injury. AJR American Journal of Roentgenology, 202(3), 614-618. doi:10.2214/AJR.13.11091

Zollman, F. S., Cyborski, C., \& Duraski, S. A. (2010). Actigraphy for assessment of sleep in traumatic brain injury: case series, review of the literature and proposed criteria for use. Brain Injury, 24(5), 748-754. doi:10.3109/02699051003692167 


\section{Glossary/Abbreviations}

AHI apnea hypopnea index

AI arousal index

APSQ Anxiety and Preoccupation about Sleep Questionnaire

CAP cyclic alternating patterns

CRSWD circadian rhythm sleep-wake disorders

DASS Depression, Anxiety, Stress Scales

DBAS Dysfunctional Beliefs and Attitudes Scale

DISRS Daytime Insomnia Symptom Response Scale

DLMO Dim Light Melatonin Onset

DSM Diagnostic and Statistical Manual for Mental Disorders

GSES Glasgow Sleep Effort Scale

EEG electroencephalography

ELISA enzyme-linked immune sorbent essay

ESS Epworth Sleepiness Scale

FSS Fatigue Severity Scale

ICSD International Classification of Sleep Disorders

ICU Intensive care unit

ISI Insomnia Severity Index

MEQ Morningness-Eveningness Scale

mTBI mild Traumatic Brain Injury

N1 stage 1 sleep

N2 stage 2 sleep 


$\begin{array}{ll}\text { N3 } & \text { stage } 3 \text { sleep (slow wave sleep) } \\ \text { OSA } & \text { obstructive sleep apnea } \\ \text { PLM } & \text { periodic leg movements } \\ \text { PLMD } & \text { periodic limb movement disorder } \\ \text { PSG } & \text { polysomnography } \\ \text { RLS } & \text { restless legs syndrome } \\ \text { SE } & \text { sleep efficiency } \\ \text { SOL } & \text { sleep onset latency } \\ \text { SWS } & \text { slow wave sleep } \\ \text { RPQ } & \text { Rivermead Post-Concussion Symptom Questionnaire } \\ \text { TBI } & \text { traumatic brain injury } \\ \text { TIB } & \text { time in bed } \\ \text { TST } & \text { total sleep time } \\ \text { VAS } & \text { Visual Analogue Scale } \\ \text { WASO } & \text { wake after sleep onset } \\ & \end{array}$

